\title{
Structural and Hydrothermal Inferences from a Magnetotelluric survey across Mt. Ruapehu, New Zealand
}

\author{
Stacey Maree Dravitzki
}

Geophysics

2005

A Research Project submitted to the School of Earth Sciences,

Victoria University of Wellington, as fulfillment for a Masters of Science, 2005.

School of Earth Sciences

Victoria University of Wellington 


\section{ABSTRACT}

This thesis explains the electrical conductivity structure of Mt. Ruapehu. To identify hydrothermal or volcanic components of the volcano, data from 25 magnetotelluric sites are analyzed.

Data collected are first analyzed in the time domain prior to conversion into the frequency domain. Here, data are remote referenced, and the impedance tensors, tippers, apparent resistivity and phase values are calculated. These components are then analyzed to identify major features within the data. The new phase tensor ellipse method is applied to identify influential features and determine the dimensionality of data. This analysis indicates where it is appropriate to apply 1 or 2 dimensional inversion schemes.

Dimensionality analysis led to 1-D modelling of the determinant impedance at each site; and limited 2-D profiles across the Tongariro Volcanic Centre boundaries. These models are used to create a simple 3-D structural model of the volcano that is then forward modelled. The results of the 3-D forward modelling indicate that the dominating features of the volcano's electrical structure have been identified in the previous models.

Crater Lake is the only possible hydrothermal system on Mt. Ruapehu identified in this study. It is also very unlikely that any large coherent bodies of magma exist in the near surface. However, a second thin conductor laying somewhere between 10 and $30 \mathrm{~km}$ deep beneath the eastern flank may contain $13 \%$ melt and is the probable driving heat force beneath the volcano.

The structure of Mt. Ruapehu can be split into seven layers.

- A resistive surface layer $(100 \Omega m)$ of young volcanic debris within the Tongariro 
Volcanic Centre that is up to $500 \mathrm{~m}$ thick near the crater.

- A conductive layer $(10-30 \Omega m)$ of wet, fractured and altered volcanic debris underlaying the younger debris throughout the Tongariro Volcanic Centre.

- A layer of Tertiary sediment under the Tongariro Volcanic Centre that extends to the south and west. This layer is electrically indistinguishable from the previous layer and extends to approximately sea level.

- A resistive layer (400 $\Omega m$ ), and consistent with greywacke basement covers the entire field area.

- A second conductive layer $(20 \Omega m)$ is identified under the eastern flank of the volcano somewhere between the depths of 10 and $30 \mathrm{~km}$. This layer is likely to be the heat and magma source driving the volcanic activity.

- A surrounding resistive layer extends beyond and below the second conductive layer mentioned above. This surrounding layer is electrically similar to the greywacke above.

- A very high resistivity layer $(7000 \Omega \mathrm{m})$ is identified below $80 \mathrm{~km}$ deep, and may be associated with the land/sea boundary or subduction zone to the east. 


\section{ACKNOWLEDGEMENTS}

Here I would like to acknowledge and thank those who spared their time, resources and/or expertise during the course of this study.

The first and biggest thank you has to go to my supervisor Dr. Malcolm Ingham. He devised the project, gave advice and provided direction during the many stages of this project, plus sis numerous other things vital to the completion of this thesis.

Secondly a huge thanks is owed to Dr. Hugh Bibby of GNS for being an unofficial "supervisor", patiently explaining many aspects of the MT theory when I confused myself. He also secured the finance, equipment and DoC permission required to undertake the fieldwork.

A big thank you is owed to all the local farmers, the Department of Conservation, Karioi Forestry Ltd, and the New Zealand Defence Force who all permitted us to occupy sites on their land.

I also need to thank all the people who dug holes and faced the lovely conditions that awaited us in February. In addition to the people mentioned above this included Stewart Bennie, Yasuo Ogawa, Grant Caldwell and Nick Horspool.

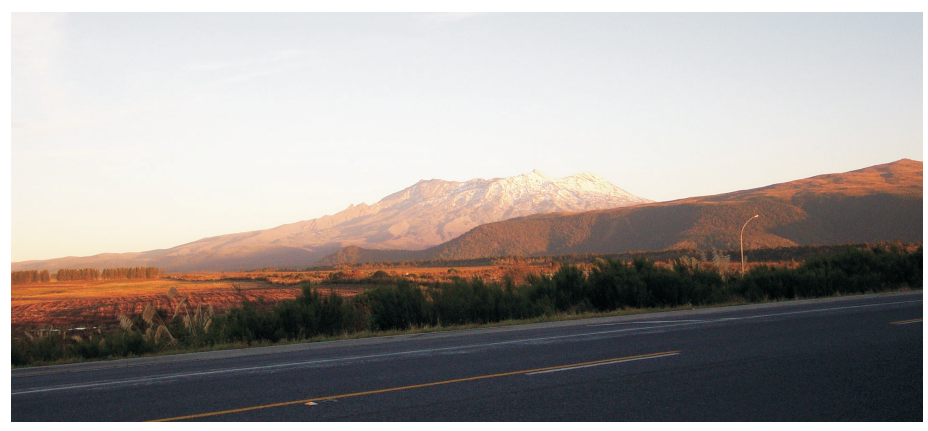

Figure 1 . Sunset view of Mt. Ruapehu with Mt Hauhungatahi in the foreground, photo taken from National Park Village.

I also utilised the computer skills of a number of people in order to get the machine to do what I wanted it to. A big thank you to all the people who patiently fixed my mistakes, and got things rolling when my own computer skills were lacking. These people include Ralph, Mike, Donald, Mark, Guy, Nick and Michelle.

Last but not least are all the people who tried to keep me sane and provided me with entertainment or someone to talk at, occasionally even about work. This is most of the people in the geophysics department, the post grad comp geek lab, Jill and the admin staff in SES, and the people in the Geonet building at GNS, and even my boyfriend, friends and family who just smiled and nodded when I started talking about this project but were there for me anyway. 


\section{CONTENTS}

$\begin{array}{ll}\text { Abstract } & \text { i }\end{array}$

Acknowledgments $\quad$ ii

Table Of Contents iii

List Of Figures vi vi

\section{Chapters}

1 Introduction 1

2 Background 4

2.1 Field Area . . . . . . . . . . . . . . . . . . . . . . . 4

2.1.1 Central Volcanic Region . . . . . . . . . . . . . . . 6

2.1.2 Taupo Volcanic Zone . . . . . . . . . . . . . . . . . . . . . . 7

2.1.3 Tongariro Volcanic Centre . . . . . . . . . . . . . . 8

2.1.4 Mt. Ruapehu . . . . . . . . . . . . . . . . 10

2.2 Resistivity Investigations on Other Volcanoes . . . . . . . . . . . . . 12

2.3 Bulk Resistivity of Rocks . . . . . . . . . . . . . . . . . . 15

$\begin{array}{llr}3 & \text { Theory } & 17\end{array}$

3.1 Electromagnetic Induction within the Earth . . . . . . . . . . . . . 17

3.1.1 1-Dimensional Earth Solutions . . . . . . . . . . . . . . . 20

3.1.2 2 and 3-dimensional Earth solutions . . . . . . . . . . . . . 23

3.2 Magnetotelluric Soundings . . . . . . . . . . . . . . . . . . . . 26

3.3 Distortion . . . . . . . . . . . . . . . . . . . . . 31

3.3 .1 Topography . . . . . . . . . . . . . . . . 32

3.3 .2 Galvanic Distortion . . . . . . . . . . . . . . . . . 33

3.3 .3 Static shift. . . . . . . . . . . . . . . . 33 
3.3.4 The Groom and Bailey Decomposition Method . . . . . . . 36

3.3.5 The Phase Tensor Method . . . . . . . . . . . . . . . . . . 37

3.4 Processing . . . . . . . . . . . . . . . . . . . . 43

3.5 Modelling . . . . . . . . . . . . . . . . . 45

4 The Data Set $\quad 48$

4.1 Data Collection and Processing . . . . . . . . . . . . 50

4.2 Apparent Resistivity and Phase Curves . . . . . . . . . . . . . . 54

4.2 .1 Group A . . . . . . . . . . . . . 56

4.2 .2 Group B ......................... 57

4.2 .3 Group C . . . . . . . . . . . . . . . 59

4.2 .4 Group D ........................... 60

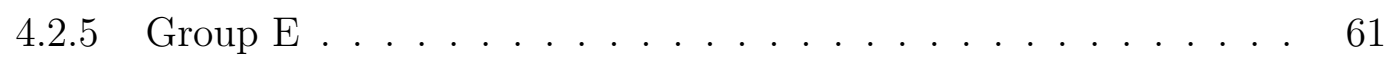

4.2 .6 Group F . . . . . . . . . . . . . . 62

4.2 .7 Conclusions . . . . . . . . . . . . . . . 63

4.3 Phase Tensor Ellipses . . . . . . . . . . . . . . . . . . 64

4.3.1 High Frequency Phase Tensor Ellipses . . . . . . . . . . . . 64

4.3.2 Dead Band Phase Tensor Ellipses . . . . . . . . . . . . . 67

4.3.3 Long Period Phase Tensor Ellipses _. . . . . . . . . . . . . . . 69

4.3 .4 Conclusions . . . . . . . . . . . . . . . . . . . 71

5 Modelling $\quad 72$

5.1 1-D Modelling . . . . . . . . . . . . . . . . . . . . . 73

5.1 .1 Group A . . . . . . . . . . . . . . . 74

5.1 .2 Group B ...................... 76

5.1 .3 Group C . . . . . . . . . . . . . 78

5.1 .4 Group D . . . . . . . . . . . . . . . . . . . . 79

5.1 .5 Group E ....................... 81

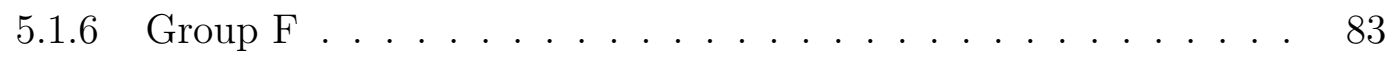

5.1 .7 Interpretation of $1-\mathrm{D}$ models . . . . . . . . . . . . . 85

5.2 2-D Modelling . . . . . . . . . . . . . . . . . . . . 88

5.2 .1 Profile A . . . . . . . . . . . . . . . . . 90

5.2 .2 Profile B . . . . . . . . . . . . . . . . . . . 92

5.3 Comparison of 1-D to the 2-D Models . . . . . . . . . . . . . . . 94

5.4 Testing the Derived Structure in $3-\mathrm{D} \ldots \ldots \ldots$. . . . . . . . . . 95 
6 Discussion 103

6.1 The Existence of Magma . . . . . . . . . . . . . . . . 103

6.2 The Structure of the Volcano . . . . . . . . . . . . . . . . . . 104

6.3 The Phase Tensor Ellipse . . . . . . . . . . . . . . . . . 107

6.4 Conclusions . . . . . . . . . . . . . . . . . . . 108

6.5 Further Work . . . . . . . . . . . . . . . . . . 110

$\begin{array}{lr}\text { References } & 111\end{array}$

\section{Appendices}

$\begin{array}{ll}\text { A Undistorted Data } & 118\end{array}$

$\begin{array}{ll}\text { B Phase Tensor Ellipse Maps } & 141\end{array}$

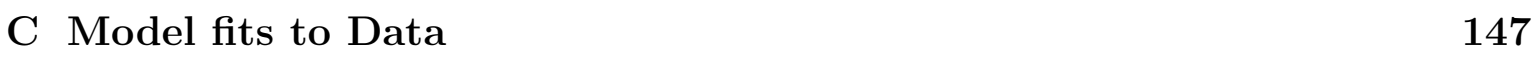

C.1 Fit of the 1-D Inversion Models . . . . . . . . . . . . . . . 147

C.2 Fit of the 2-D Inversion Models . . . . . . . . . . . . . . 153

C.3 Data fit for 3-D Test . . . . . . . . . . . . . . . 155

$\begin{array}{ll}\text { D Symbols Used } & 161\end{array}$ 


\section{FIGURES}

1 Sunset view of Mt. Ruapehu . . . . . . . . . . . . . . . . iii

2.1 Important structural boundaries in the North Island . . . . . . . . . . . 5

2.2 Recent volcanic centres in the Central Volcanic region and Taupo Volcanic Zone . . . . . . . . . . . . . . . . . . . . . 6 6

2.3 Depth to the greywacke basement as obtained from drill holes at Ohaaki Geothermal field . . . . . . . . . . . . . . . . 7

2.4 A simplistic map of the Tongariro Volcanic Centre . . . . . . . . . . . . 9

2.5 Schematic stratigraphic models of Mt. Ruapehu . . . . . . . . . . . . . 11

3.1 Development of boundary conditions in MT theory . . . . . . . . . 25

3.2 The effects of inhomogenieties on electric fields . . . . . . . . . . . . . 34

3.3 The parameters of the phase tensor ellipse . . . . . . . . . . . . . 40

4.1 The level of terrestrial magnetic activity recorded during fieldwork . . . 49

4.2 The location of MT sites occupied during the 2004 Mt Ruapehu MT survey ........................... 50

4.3 An example of the graphs created by plotting the data and phase tensor parameters . . . . . . . . . . . . . . . 5 55

4.4 Grouping boundaries for sites with similar apparent resistivity and phase

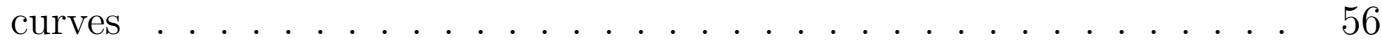

4.5 An apparent resistivity and phase curve in Group A . . . . . . . . . . 57

4.6 An apparent resistivity and phase curve in Group B . . . . . . . . . . 58

4.7 An apparent resistivity and phase curve in Group C . . . . . . . . . . 59

4.8 An apparent resistivity and phase curve in Group D . . . . . . . . . . . 60

4.9 An apparent resistivity and phase curve in Group E . . . . . . . . . . . 62

4.10 An apparent resistivity and phase curve in Group F . . . . . . . . . . . 62

4.11 High frequency phase tensor ellipse map . . . . . . . . . . . . . 65

4.12 The effects of topography on high frequency phase tensor ellipses . . . 66

4.13 Dead band phase tensor ellipse map . . . . . . . . . . . . . . . . 68

4.14 Long period phase tensor ellipse map . . . . . . . . . . . . . . . 70 
5.1 Grouping of sites which produced similar 1-D models and the location of the 2-D profile lines . . . . . . . . . . . . . . . . . . . 72

5.2 Depth plot of 1-D models produced in group A . . . . . . . . . . 74

5.3 Near surface structure from 1-D models produced in group A . . . . . 74

5.4 Depth plot of 1-D models produced in group B . . . . . . . . . . 76

5.5 Near surface structure from 1-D models produced in group B . . . . . . 77

5.6 Depth plot of 1-D models produced in group $\mathbf{C} \ldots \ldots$. . . . . . . 78

5.7 Near surface structure from 1-D models produced in group C . . . . . . 78

5.8 Depth plot of 1-D models produced in group D . . . . . . . . . . 80

5.9 Near surface structure from 1-D models produced in group D . . . . . 80

5.10 Depth plot of 1-D models produced in group $\mathbf{E} \ldots \ldots$. . . . . . . . 82

5.11 Near surface structure from 1-D models produced in group E . . . . . . 82

5.12 Depth plot of 1-D models produced in group F . . . . . . . . . . 83

5.13 Near surface structure from 1-D models produced in group F . . . . . . 84

5.14 The height difference between the top of the shallow conductor and the site altitude. . . . . . . . . . . . . . . 86

5.15 Contour map of the top surface of the shallow conductor identified in 1-D models. . . . . . . . . . . . . . . . . . . 87

5.16 The 2-D model produced from Profile A . . . . . . . . . . . . . 91

5.17 The 2-D model produced from Profile B . . . . . . . . . . . . . . 93

5.18 Griding of steep topography to obtain the best fit to actual data . . . . 96

5.19 An east/west cross section from the 3-D mesh used to test the validity of the structure . . . . . . . . . . . . . . . . 96

5.20 A horizontal cross section from the 3-D mesh used to test the validity of the structure . . . . . . . . . . . . . . . . . 97

5.21 Two examples of apparent resistivity and phase curves determined from the results of the 3 -D forward model . . . . . . . . . . . . . . . . 98

5.22 Phase tensor ellipses derived from the results of the original 3-D forward

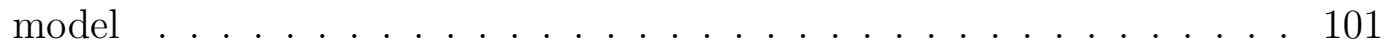

A.1 Data from site MTR-101a . . . . . . . . . . . . . . . . . . . . 119

A.2 Data from site MTR-102a . . . . . . . . . . . . . . . 120

A.3 Data from site MTR-103a . . . . . . . . . . . . . . . . . . . 121

A.4 Data from site MTR-104a . . . . . . . . . . . . . . . . . . . 122

A.5 Data from site MTR-105a . . . . . . . . . . . . . . . . . 123

A.6 Data from site MTR-106a . . . . . . . . . . . . . . . . . . . 124

A.7 Data from site MTR-107a . . . . . . . . . . . . . . . . 125 
A.8 Data from site MTR-110a . . . . . . . . . . . . . . . . . 126

A.9 Data from site MTR-111a . . . . . . . . . . . . . . . . . 127

A.10 Data from site MTR-112a . . . . . . . . . . . . . . . . . . . . 128

A.11 Data from site MTR-113a . . . . . . . . . . . . . . . . . . . . . 129

A.12 Data from site MTR-114a . . . . . . . . . . . . . . . . 130

A.13 Data from site MTR-115a . . . . . . . . . . . . . . . . 131

A.14 Data from site MTR-116a . . . . . . . . . . . . . . . . . . 132

A.15 Data from site MTR-117a . . . . . . . . . . . . . . . . . 133

A.16 Data from site MTR-118b . . . . . . . . . . . . . . . . . . 134

A.17 Data from site MTR-119a . . . . . . . . . . . . . . . . . 135

A.18 Data from site MTR-120a . . . . . . . . . . . . . . . . . . 136

A.19 Data from site MTR-121a . . . . . . . . . . . . . . . 137

A.20 Data from site MTR-122a . . . . . . . . . . . . . . . . . . . . . . 138

A.21 Data from site MTR-123a . . . . . . . . . . . . . . . . . . 139

A.22 Data from site MTR-124a . . . . . . . . . . . . . . . . . 140

B.1 Phase tensor ellipse map: frequency $=240 \mathrm{~Hz} \ldots \ldots \ldots$. . . . . . . 141

B.2 Phase tensor ellipse map: frequency $=60 \mathrm{~Hz} \ldots \ldots \ldots \ldots$

B.3 Phase tensor ellipse map: frequency $=15 \mathrm{~Hz} \ldots \ldots$. . . . . . . . . . 142

B.4 Phase tensor ellipse map: frequency $=4.5 \mathrm{~Hz} \ldots \ldots \ldots \ldots$

B.5 Phase tensor ellipse map: frequency $=1.125 \mathrm{~Hz}$. . . . . . . . . . . . 143

B.6 Phase tensor ellipse map: frequency $=0.281 \mathrm{~Hz} \ldots \ldots$. . . . . . . . 144

B.7 Phase tensor ellipse map: period $=14.222 \mathrm{~s} \ldots \ldots \ldots \ldots$. . . . . 144

B.8 Phase tensor ellipse map: $\operatorname{period}=56.889 \mathrm{~s} \ldots \ldots \ldots \ldots$

B.9 Phase tensor ellipse map: period $=227.556 \mathrm{~s} \ldots \ldots \ldots \ldots$

B.10 Phase tensor ellipse map: period $=910.222 s \ldots \ldots$. . . . . . 146

C.1 Theoretical fit of 1-D models (sites MTR-101a to MTR-102a) . . . . . 147

C.2 Theoretical fit of 1-D models (sites MTR-103a to MTR-106a) . . . . . 148

C.3 Theoretical fit of 1-D models (sites MTR-107a to MTR-112a) . . . . . 149

C.4 Theoretical fit of 1-D models (sites MTR-113a to MTR-116a) . . . . . 150

C.5 Theoretical fit of 1-D models (sites MTR-117a to MTR-120a) . . . . . 151

C.6 Theoretical fit of 1-D models (sites MTR-121a to MTR-124a) . . . . . 152

C.7 Theoretical fit of derived data from Profile A to observed data . . . . . 153

C.8 Theoretical fit of derived data from Profile B to observed data . . . . . 154 
C.9 Theoretical fit of 3-D forward model (sites MTR-101a to MTR-102a) · 155 C.10 Theoretical fit of 3-D forward model (sites MTR-103a to MTR-106a) 156 C.11 Theoretical fit of 3-D forward model (sites MTR-107a to MTR-112a) 157 C.12 Theoretical fit of 3-D forward model (sites MTR-113a to MTR-116a) 158 C.13 Theoretical fit of 3-D forward model (sites MTR-117a to MTR-120a) . 159 C.14 Theoretical fit of 3-D forward model (sites MTR-121a to MTR-124a) 160 


\section{CHAPTER 1}

\section{INTRODUCTION}

Although Mt. Ruapehu is a large dominating active volcano in New Zealand very little is known about its internal structure. Vast amounts of geological data have been collected and interpreted from the surface lava flows and very detailed compositions of these lavas have been documented, but the location of any magma chamber(s) and the structure beneath the volcano are still largely undefined.

One aim of this study is to use the deep electrical conductivity structure of the volcano to add further information to the lithological sequence and extent of subsurface layers. Previous geophysical studies on Mt. Ruapehu have produced non-unique models of possible structures. To date no definite conclusion has been reached about the presence of Tertiary sediment beneath Mt. Ruapehu. A shallow seismic reflection survey to the southeast of Mt. Ruapehu (Sissons and Dibble, 1981) indicated velocities compatible with Tertiary sediment, and the authors then modelled a Tertiary sediment layer beneath the pyroclastic material. However, gravity models created by Zeng (1996) contained a thicker pyroclastic layer; eliminating the need for a Tertiary sediment layer. Tertiary sediment outcrops beneath the lava cap at Mt. Hauhungatahi as well as to the south and west of the Tongariro Volcanic Centre (TVC) (Hay, 1967), this strongly suggest that this layer extends beneath Mt. Ruapehu. One of the aims of this thesis is to provide more constraint on these subsurface layers.

Electric geophysical methods have been widely used in the detection and delineation of hydrothermal and magmatic systems associated with volcanoes in the past (e.g. Manzella et al. (2004); Matsushima et al. (2001); Ogawa et al. (1998)). Hot or molten bodies are usually identified by extremely low resistivity (Hermance and Colp, 1982). 
Tertiary sediments have been similarly identified by their low resistivity (e.g. Ingham et al. (2001)). Therefore, with the primary aim of both investigating the deep structure and identifying any electrically conductive features associated with hydrothermal or volcanic activity, a magnetotelluric (MT) survey of the volcano was conducted in early 2004. Twenty-four MT sites were occupied on and around the volcano and these data were combined with earlier long period data collected by Hanekop (2002) at three additional locations.

The secondary aim of this study was to test the application of a new distortion analysis technique for MT data. Analysis of distortion using the MT phase tensor (Caldwell et al., 2004) has been used in conjunction with a distortion stripping technique developed by Bibby et al. (In Press). This new method does not require the regional 2-D assumption that is inherent in most other distortion removal techniques. Mt. Ruapehu is a 3-D feature and this new method provides a way to remove galvanic effects without losing the 3-D nature of the data. Spatial plots of the phase tensor ellipses quickly and easily show how the dimensionality and directionality of current flow varies as a function of frequency. The ellipse maps have been used in this study to identify 1 and 2-D features influencing electromagnetic (EM) wave induction; thus identifying where 1 or $2-\mathrm{D}$ modelling is appropriate.

This thesis has been divided into a number of chapters discussing the processes and data involved in reaching the aims stated above. These chapters are outlined as below:

- The background chapter presents a summary of some of the previous geological and geophysical work within the TVC and surrounding areas. It also provides a summary of previous EM studies conducted in similar volcanic environments, and explores the practical limitations of EM fieldwork.

- Chapter 3 presents a summary of EM induction theory. This chapter describes the application of EM theory to MT studies, data processing, and distortion removal for MT data. Due to the application of a new distortion analysis technique previous methods have been summarized and compared to the new phase tensor method. 
- At this point the thesis moves on to discuss the data collected in this survey, specific site locations and the processing that took place. I then present and interpret the corrected apparent resistivity and phase curves, as well as the phase tensor ellipse maps. This chapter aims to identify the dimensionality and dominant structures influencing EM wave induction beneath Mt. Ruapehu.

- With an understanding of the influencing structures and dimensionality, a number of numerical models were created to quantify the structures identified in the previous chapter. This chapter presents the models along with their interpretation and a comparison between the 1 and 2-D models created. From this a simplified 3-D conductivity structure was created and tested using a forward modelling program to test the validity of the structure defined in earlier models.

- Concluding this thesis is a discussion chapter that compares the model interpretations with the existing geophysical knowledge. Lastly combining this knowledge with the models presented in this thesis in an attempt to answer the questions that motivated this survey. 
CHAPTER 2

\section{BACKGROUND}

Mt. Ruapehu was chosen as the field area for this magnetotelluric (MT) study as very little is known about the internal structure of this active volcano. Previous MT investigations overseas have successfully enhanced the knowledge of the internal structure of many volcanoes (e.g. Merapi, Indonesia (Müller and Haak, 2004); Mt. SommaVesuvius, Italy (Manzella et al., 2004); and Usu, Japan (Matsushima et al., 2001)to name a few). A basic understanding of the geologic and tectonic settings and history of the field area are required in order to interpret the results realistically.

Mt. Ruapehu is located in the central North Island of New Zealand and since James Park first described the summit in 1887 a vast amount of research has been completed to understand the history, composition and processes driving this volcano. This chapter summarizes the key knowledge that needs to be considered in the development and understanding of an MT interpretation of Mt. Ruapehu.

The last sections of this chapter discuss the solutions and limitations of the MT method that need to be considered throughout the interpretation of this data set.

\section{$2.1 \quad$ Field Area}

New Zealand forms the southern section of the Pacific/Australian tectonic plate boundary zone (shown in figure 2.1). The section of the boundary passing through New Zealand is the collision of a west dipping subduction zone in the North Island and an east dipping subduction zone south of the South Island. These subduction zones are joined by the large strike slip Alpine Fault through the South Island. The northern 


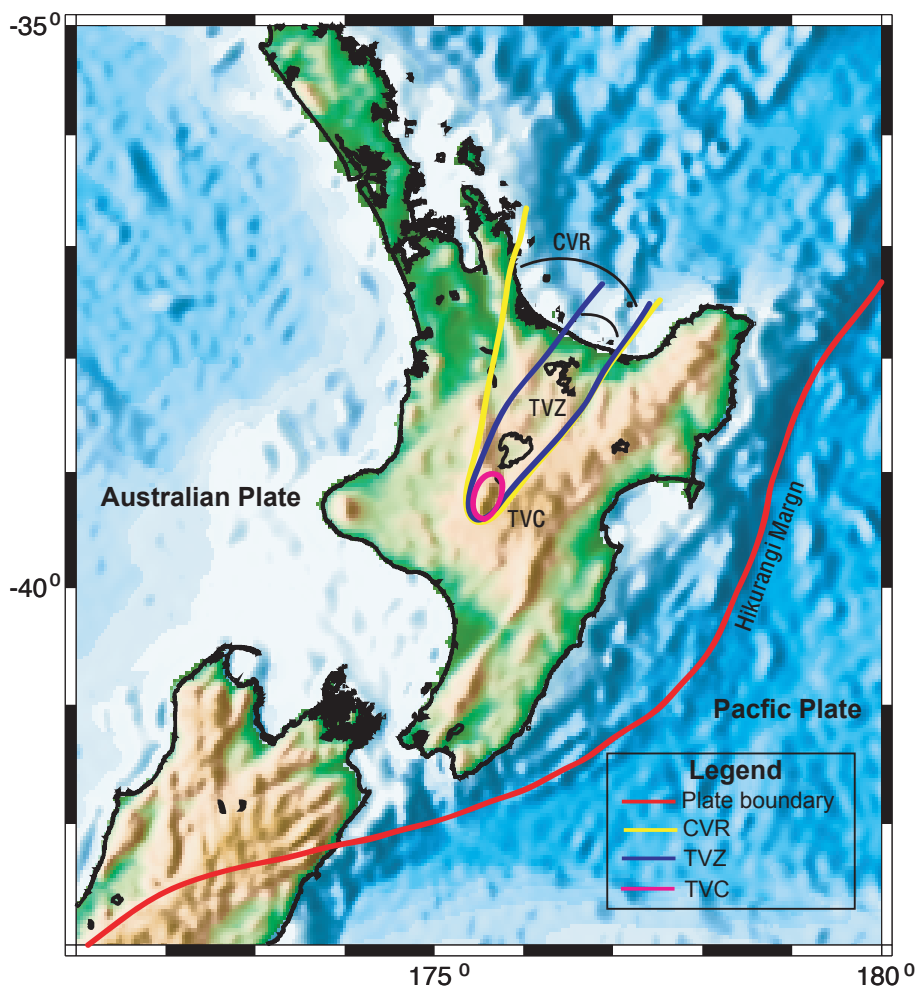

Figure 2.1 Important structural boundaries in the North Island.

subduction zone (the Hikurangi margin, described in detail in Smith et al. (1989)) is associated with North Island volcanics.

The Hikurangi margin is the southern extension of a $2800 \mathrm{~km}$ long chain of active convergent margin stretching from Tonga to Mt. Ruapehu (Ewart et al., 1977). This margin has associated back arc volcanism along the full extent of subduction. Although volcanism is present, Ewart et al. (1977) showed that volcanoes on continental New Zealand have differing lava compositions and history to many of the margin's other volcanoes.

New Zealand volcanism has been further sub-grouped into a number of sections relating to the history and composition of the volcanism. Mt. Ruapehu is the southern hinge of the Central Volcanic Region (CVR), the Taupo Volcanic Zone (TVZ), and the Tongariro Volcanic Centre (TVC). The extent of these areas are shown in figure 2.1. As the zones become smaller the defining characteristics become more detailed, thus the zones are described here in descending size order. 


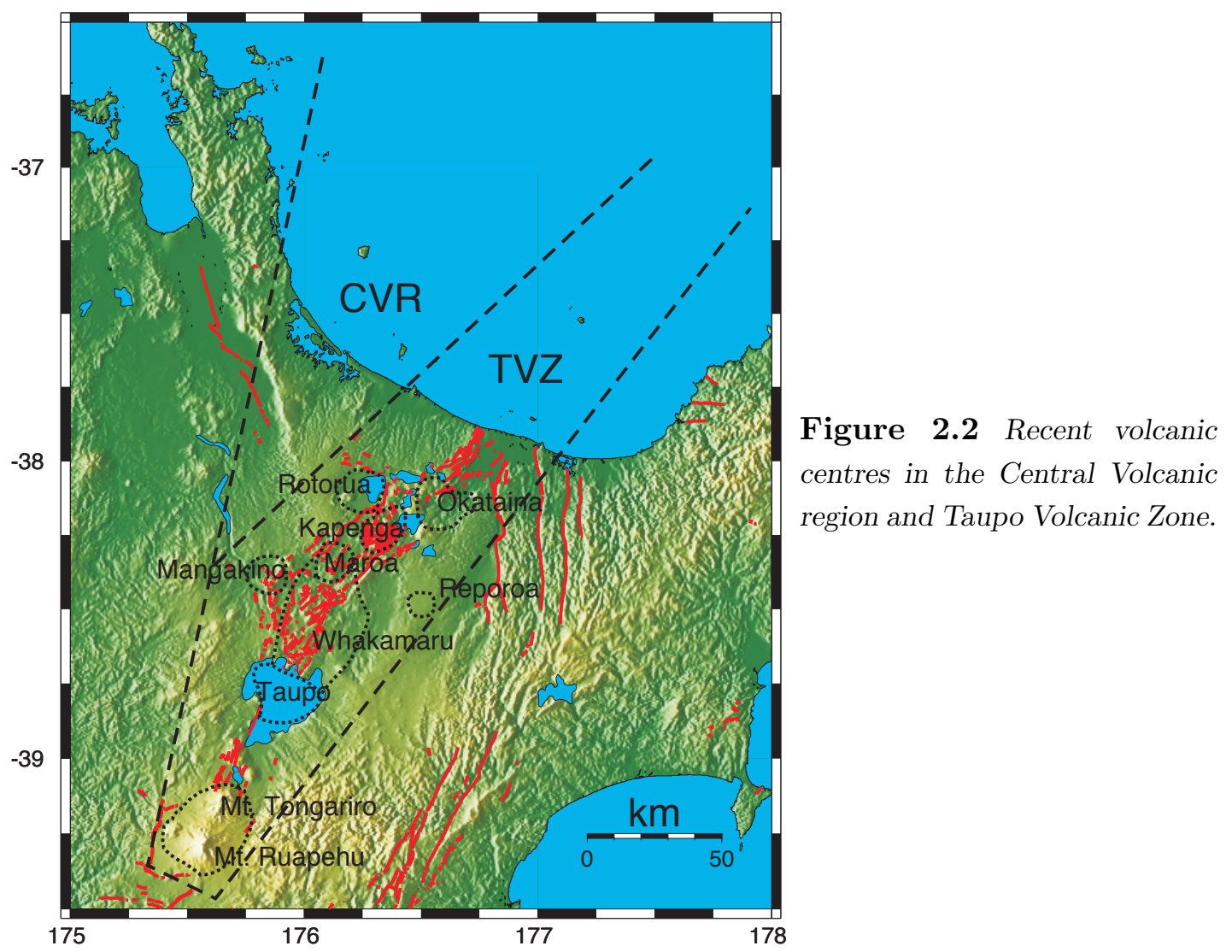

\subsubsection{Central Volcanic Region}

The CVR (refer to figure 2.2) is "a wedge-shaped area, defined by gravity data and limited age data. It shares a common eastern boundary with the TVZ but extends further to the northwest" (Wilson et al., 1995). This region is predominantly a collapse feature where volcanic material associated with the current subduction zone has been identified. The terms CVR and TVZ were poorly defined for a long time and some authors used properties of the TVZ to describe the CVR. The difference between the areas (according to the definitions applied in this thesis) are identified in figure 2.2. In recent literature the term CVR is not commonly used.

In the section of the CVR that is not included in the TVZ there are Tertiary and Quaternary volcanics interspersed amongst older rocks including large areas of Mesozoic sediment. In contrast, the TVZ is almost entirely occupied by Quaternary volcanics. The only exposed rocks older than late Tertiary are in three small outcrops in Bay of Plenty (Calhaem, 1973). Kear (1959, cited in Calhaem (1973)) stated that the centre of volcanism has migrated towards the trench as the Benioff Zone has steepened; 
with current activity occurring beneath the TVZ.

\subsubsection{Taupo Volcanic Zone}

The TVZ is defined by the envelope containing all Quaternary volcanic centres of the central North Island (Wilson et al., 1995). It could alternatively be defined as the envelope containing all high temperature geothermal systems (Bibby et al., 1995).

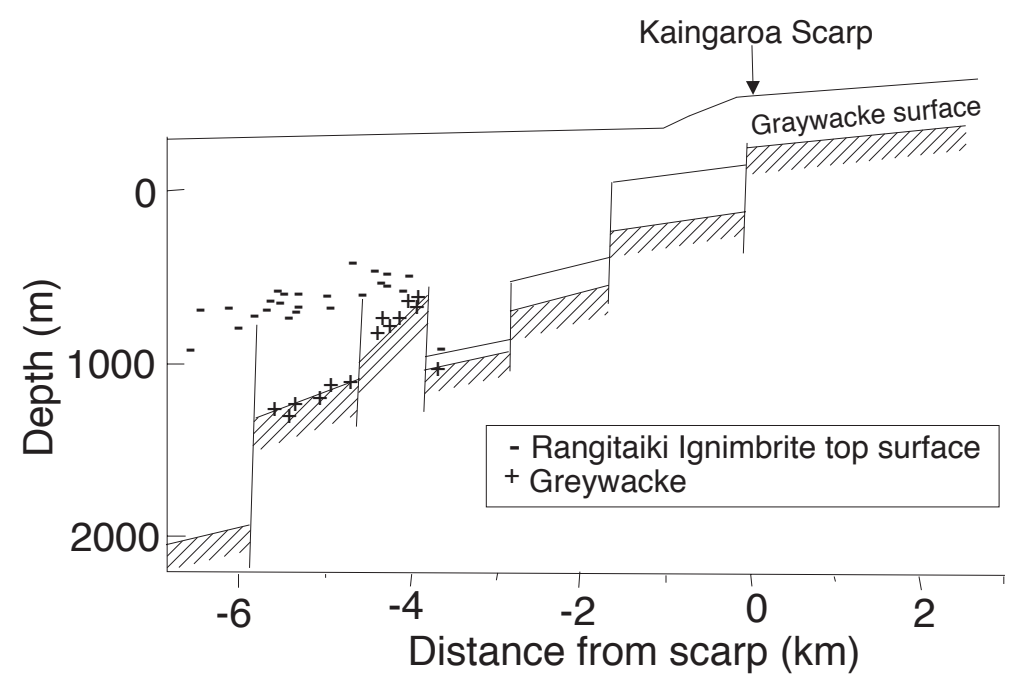

Figure 2.3 Depth to the greywacke basement and 330 ka Rangitaiki Ignimbrite obtained from drill holes at Ohaaki geothermal field, sites have been projected onto a line perpendicular to the eastern margin of the TVZ. The buried surfaces shown to the east of the drill holes are schematic only and are inferred from interpretations of seismic and gravity data. Figure taken from Bibby et al. (1995).

The eastern boundary of the TVZ is shared with the CVR, and is also marked by a height change in the basement greywacke. East of the TVZ outcrops of greywacke basement are common. Near Ohaaki geothermal field, drill holes indicated that the basement is displaced by a series of vertical faults with the basement generally being down-faulted to the west as in figure 2.3. Further from the boundary, drill holes 2.7 $\mathrm{km}$ deep did not penetrate the volcanic succession (Wilson et al., 1995).

The TVZ contains at least 34 caldera-forming ignimbrite eruptions producing a complex sequence of relatively short-lived, nested and/or overlapping volcanic centres dating back 1.6 Ma (Houghton et al., 1995). The large Whakamaru group erupted $\approx 0.34 \mathrm{Ma}$ covering the entire TVZ and acts as an age marker. 
The mode of volcanism varies along the length of the TVZ from andesitic dominated in the south (the TVC) giving way to rhyolitic dominated in the central region, returning to andesitic north of Kawerau. The volcanics in the rhyolitic dominated area are characterized by large caldera collapse zones, eight of these features have been identified (Wilson et al., 1995).

In addition to volcanism, the central North Island is undergoing back arc spreading. The extension is hinged in the south near Ohakune where extension is minimal, although average extension rates of $8 \mathrm{~mm} / \mathrm{yr}$ are recorded in the central TVZ (Darby et al., 2000). The crustal extension is being accommodated through a thinning of the crust as opposed to new crustal material being injected into surface splits. (Pulford and Stern, 2004). The up welling mantle displaces parts of the asthenosphere resulting in a geophysically anomalous lower crustal environment.

The anomalous features of the TVZ include:

- A low velocity zone at $15 \mathrm{~km}$ deep; originally this layer was thought to be the crust/mantle boundary (Stern and Davey, 1987), however recent studies have re-calculated the crust/mantle boundary to be $\approx 30 \mathrm{~km}$ deep (Bannister et al., 2004; Stratford and Stern, 2004).

- A slow upper mantle seismic velocity of $7.5 \pm 0.2 \mathrm{~km} / \mathrm{s}$; compared with the standard value of $8.1 \mathrm{~km} / \mathrm{s}$.

- High uplift and exhumation rates (Pulford and Stern, 2004); up to $2.5 \mathrm{~km}$ of uplift has occurred in the central North Island.

- Heat flow rates 12 times greater than normal continental values (Stern and Davey, 1987); the average heat flow value is $\approx 700 \mathrm{~mW} / \mathrm{m}^{2}$ for the central TVZ (Bibby et al., 1995).

\subsubsection{Tongariro Volcanic Centre}

The TVC is the southern part of the CVR/TVZ containing recent active andesitic volcanism, and includes five andesite massifs; Ruapehu, Tongariro, Ngauruhoe, Pihanga 
and Kakaramea, plus many smaller cones and flows (Cole, 1978). Mt. Tongariro is slightly to the north of Mt. Ruapehu, and lavas forming the saddle between the volcanoes have been identified as the oldest lavas from Tongariro (Stipp, 1968, cited from Zeng (1996)) Although smaller than Mt. Ruapehu, Tongariro with its side peak of Ngauruhoe is a considerable landmass that will effect some of the data collected in this survey.

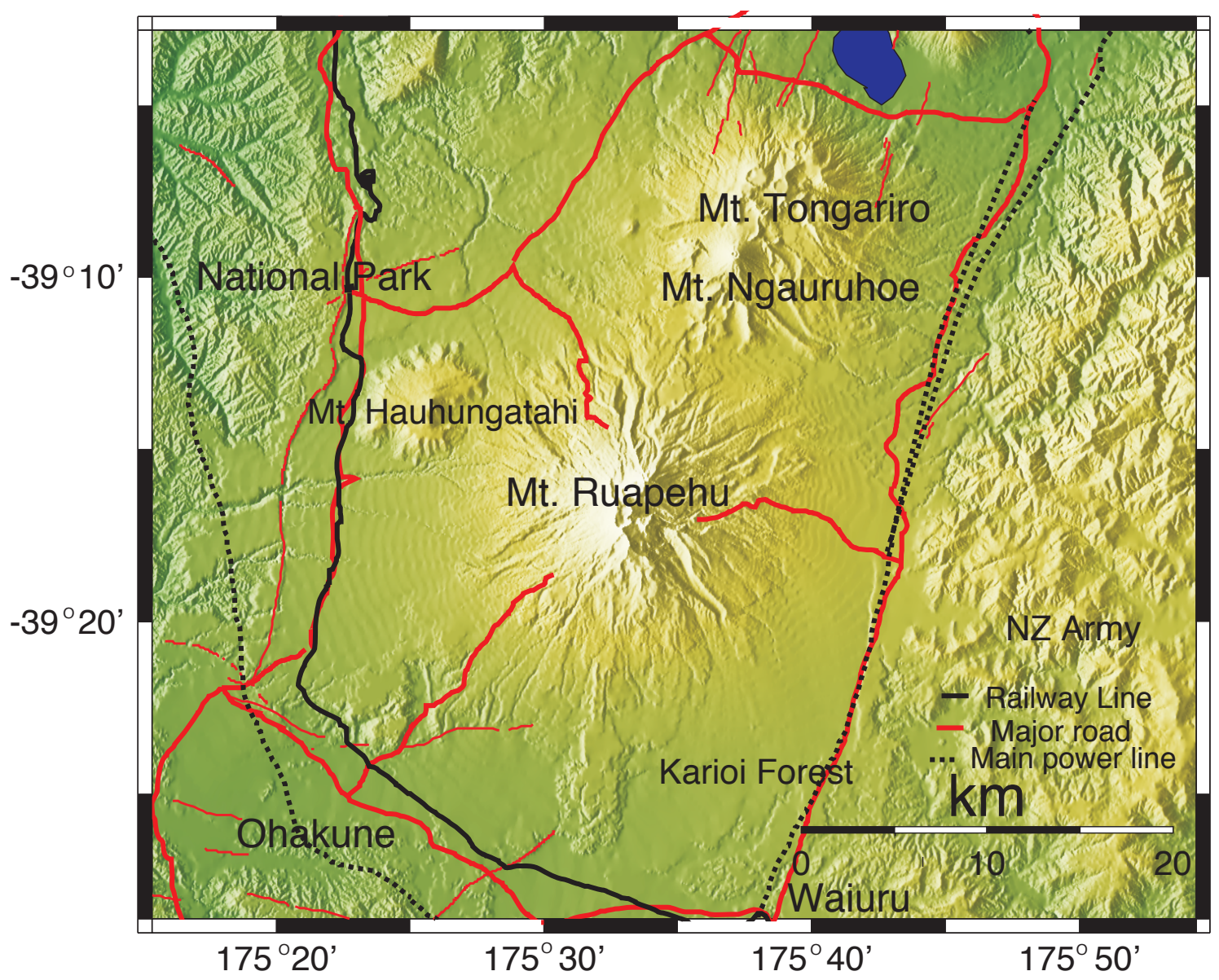

Figure 2.4 A simplistic map of the Tongariro Volcanic Centre showing the main surface features of the area.

The near surface geology of the TVC is a complex system of lava dome extrusions, aa and block lava flows, tephra and pyroclastic debris (Hackett, 1985). The separate deposits often have slightly different chemical compositions and were deposited during different eruptive events (Topping, 1974). Geologists have dedicated years of research into the identification and classification of these individual deposits in order to accurately define the eruptive history of the volcanoes. Although this knowledge puts 
constraints on surface layers, the resolution of MT data are insufficient to distinguish between separate event deposits.

The deeper layers that are distinguishable through MT may have also been identified during previous geophysical studies. A selection of these studies have been consulted in the discussion and interpretation of the current study (refer to Chapter 6); these include Hanekop (2002); Hurst and McGinty (1999); Zeng (1996); Hurst et al. (1991); Ingham (1987); Olson (1985); Alder (1983); Latter (1981); Sissons and Dibble (1981); and Marriott (1969).

Predominately, faults within the TVC are normal and usually dip towards the eruptive centres (Cole, 1990). The main boundary faults are the Waimarino and Desert Road Faults. The Desert Road Fault is also known as the Rangipo Fault and is a prominent north-northeast striking, west dipping, active normal fault that defines the eastern boundary at the southern termination of the TVZ (Villamor et al., 2004).

Cross sections of the Waimarino Fault (also known as the Raurimu Fault) indicate that Tertiary sediment was down faulted on the eastern side. The amount of dip slip has been debated. Lecointre et al. (1998) gave a value of $140 m$ dip slip. Meanwhile Pulford and Stern (2004) estimated the dip slip at $200 \mathrm{~m}$ based on an unpublished gravity survey, and Hurst and McGinty (1999) estimated the offset at only $60 \mathrm{~m}$. Although the amount of slip is debated it is agreed that the movement has occurred over the last 60,000 years, coinciding with recent volcanism.

Olson (1985) deduced from passive seismology that no large magma body or substantial partial melt could exist between Ngauruhoe and Ruapehu. Indeed no seismic nor electrical study has provided evidence of high temperature melt at shallow depth within the TVC at any location.

\subsubsection{Mt. Ruapehu}

Mt. Ruapehu is a large complex andesitic strato-volcano, rising to $2797 \mathrm{~m}$ (asl) above the surrounding ring plain at $1200 \mathrm{~m}$ (asl). Tertiary sediment surrounding the volcano contain no volcanic material (Fleming and Steiner, 1951), yet early Quaternary conglomerates south of Mt. Ruapehu contain andesite pebbles (Dibble, 1972). These 
suggest that volcanism originated during the Quaternary. Four dominantly andesitic cone building episodes have been identified over the last $250 \mathrm{ky}$ (Luther, 1999; Hackett, 1985). Geologically the cone is $\approx 110 \mathrm{~km}^{3}$ of sheet and autobrecciated lava flows, surrounded by an equally voluminous ring plain composed of products from explosive pyroclastic eruptions and reworked detrital material (Luther, 1999).

Recently the crater has had a pattern of intermittent hydrothermal-magmatic eruptions. Violent eruptions have been recorded in 1952, 1969, 1971, 1975 and most recently in 1995 and 1996. Prior to the 1995-6 eruption the vent system was in an apparently stable cycle of short heating episodes followed by longer quiescent phases. This was explained by Hurst et al. (1991), as indicating that the vent system was hidden beneath the hot acidic lake and interacted with the underlying vent system in a heat pump manner. The lake waters and their surrounding drainage will have an extremely low resistivity, reducing apparent resistivities recorded nearby.

Simplified stratigraphic structures (figure 2.5) of the volcano as derived from seismic studies (Olson, 1985; Latter, 1981; Sissons and Dibble, 1981) show that the structure can be split into three main units; volcanic, sedimentary, and basement layers.

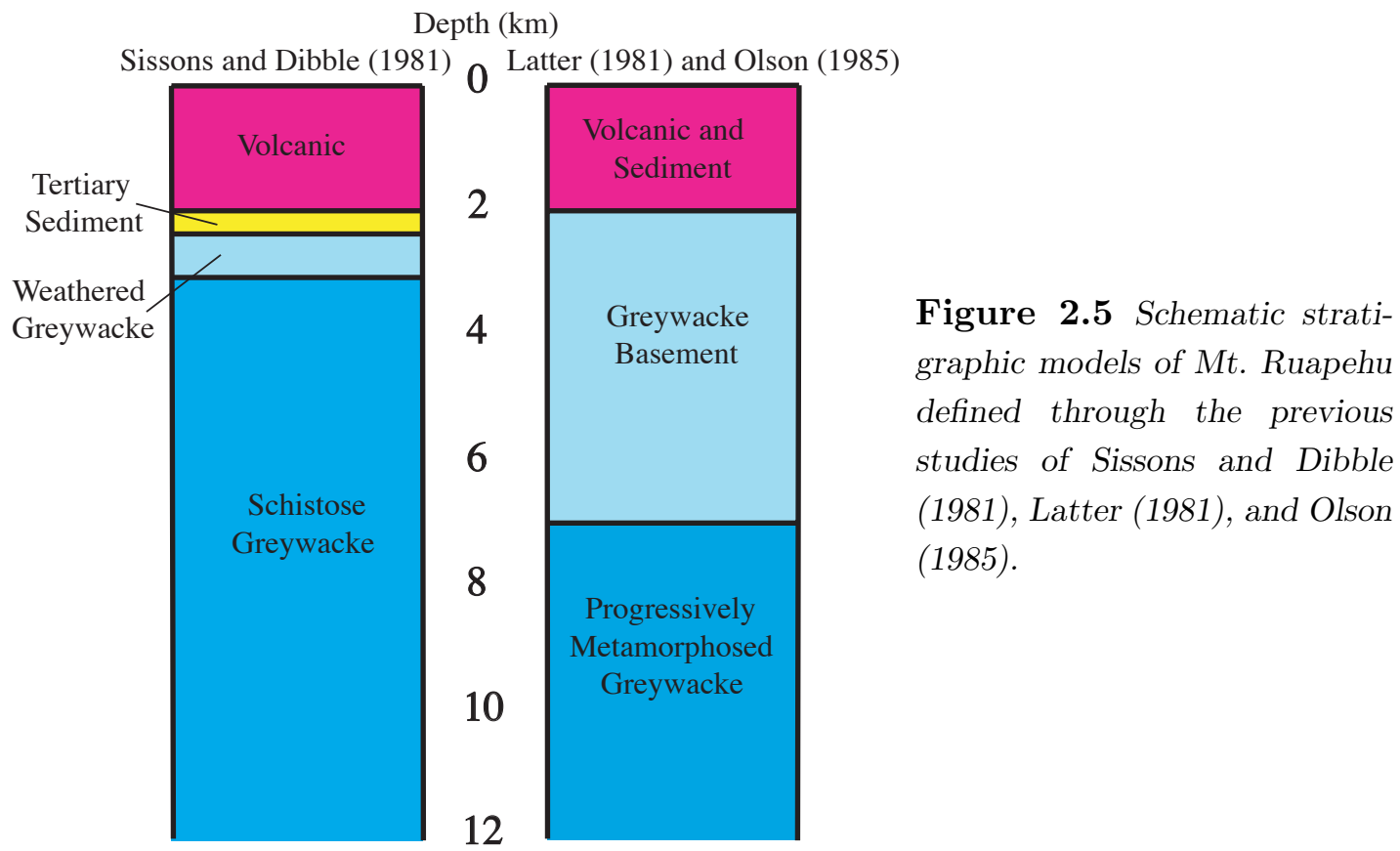

In all three seismic studies, the top $2 \mathrm{~km}$ of material was attributed to volcanic 
eruptives. Of these studies only Sissons and Dibble (1981) distinguished a boundary between the volcanic debris and sediment. The authors asserted that this sediment is of Tertiary age. The physical properties of these two lithologies are similar and therefore a boundary between them is difficult to distinguish. Other geophysical models also have differently classified these units. Gravity data can be modelled with a thicker pyroclastic layer instead of a Tertiary sediment layer (Zeng, 1996) or at a lower density (indicating a higher proportion of pyroclastic material) (Horspool, 2003) without reducing the fit of the model.

The multiple greywacke layers seen in figure 2.5 account for the progressive metamorphosis of the basement as was necessitated through the gradual increase in seismic velocity with depth. Evidence that this basement layer is indeed greywacke was provided by the identification of greywacke xenoliths in Ruapehu lavas (Grange and Williamson, 1930, cited in Olson (1985)).

In addition to the properties identified here that will influence the apparent resistivity values recorded on Mt. Ruapehu, a number of similar MT studies have been conducted on volcanoes worldwide. These international studies will also help in the interpretation of the features identified in this current study.

\subsection{Resistivity Investigations on Other Volcanoes}

The use of electromagnetic methods to interpret the structure in volcanic environments is wide spread. MT methods have been conducted in the following locations:

- Central Andes, Chile (Schilling et al., 1997),

- Kilauea Iki, USA (Hermance and Colp, 1982),

- Kuju Volcano, Japan (Mogi and Nakama, 1993),

- Long Valley, USA (Park and Torres-Verdin, 1988),

- Merapi Volcano, Indonesia (Müller and Haak, 2004),

- Mount Hood, USA (Mozley et al., 1986), 
- Mt. Ruapehu, New Zealand (Hanekop, 2002; Zeng, 1996),

- Mt Somma-Vesuvius, Italy (Manzella et al., 2004),

- Norikura Volcano, Japan (Fuji-ta et al., 1999),

- Unzen Volcano, Japan (Kagiyama et al., 1999),

- Usu Volcano, Japan (Ogawa et al., 1998; Matsushima et al., 2001),

- White Island, New Zealand (Ingham, 1992).

These studies have determined the characteristics of common volcanic features, and of distortion sources that regularly interfere with data signals in these environments. Furthermore, laboratory measurements have added constraints on resistivity values of lithological units likely to be found in volcanic areas.

One common feature in volcanic environments is hydrothermal fluid or melt, identified by its low resistivity. In a laboratory Haak (1980, cited in Müller and Haak (2004)) calculated the resistivity of total melt to be $0.2 \Omega m$. In situ values for total melt have been recorded up to $2.5 \Omega m$ (Hermance and Colp, 1982). The difference between the resistivity values maybe explained by the difference between the properties of degassed and non-degassed magma.

Since distribution, proportion and connectivity of the magma all influence the apparent resistivity recorded, it is unlikely that a magma chamber will have an apparent resistivity equal to that of pure melt. Geothermal areas also have anomalously low resistivities (Bibby et al., 1998; Ogawa et al., 1998), even where there are no active volcanic vents. Hot saline geothermal fluids and clays could easily be mistaken for magma in a volcanic survey due to comparable resistivity values (Müller and Haak, 2004).

A low resistivity value may be due to the heat and chemistry of the fluids as opposed to properties of the rock type. This discrepancy needs to be considered when identifying conductive features within a volcano. On the other hand, high resistivity in volcanic environments usually represent unaltered rocks that have never hosted hot thermal waters (Risk et al., 1994). 
As hot active material is easily identified in MT surveys, this technique has been proposed as a tool for volcanic monitoring. Just prior to an eruption, MT might identify and possibility assess the speed of rising magma and distinguish ground water boundary layers. The electrical structure can identify areas of fluid saturation and improve interpretation of hazard monitoring techniques. Ground water has been recognized as an eruption trigger and is known to influence phreatic eruptions (Kagiyama et al., 1999; Houghton et al., 1987; Latter, 1981; Cole, 1978).

On large topographical features such as a volcano, topographic effects cannot be ignored. Traditionally, large scale topography is dealt with during the modelling phase, while small scale topography is generally ignored or classed as part of the galvanic distortion. There are several ways of modelling topography; Ogawa et al. (1998) and Fuji-ta et al. (1999) ignored topography during the inversion, but when the model was presented the grid squares were transformed to parallelograms to represent the shape of the topography. Alternatively, Müller and Haak (2004) retained a horizontal and vertical grid structure and used the grid to create a stepping structure prior to inversion calculations.

On volcanoes the radial component (component representing radial direction from the centre of the volcano) of the impedance tensor can be one to two orders of magnitude larger than the tangential component (orthogonal direction) due to small scale radial ridges on volcanoes (Müller and Haak, 2004). This is because an electrical current prefers to flow through rock as it has a much lower resistivity than air. If a 3-D model is created using a rectangular grid then this effect is very difficult to model.

Remote referencing is the process of using a distant quiet site to remove local noise from MT data. Unfortunately remote referencing is not as effective at removing noise in mountainous environments. There are greater magnetic field variations and higher noise:signal ratios (Ádám et al., 1986) near volcanoes. Any distortion that is not removed during the remote referencing needs to be removed from the data prior to any modelling if a realistic model is to be obtained.

In addition to these conductivity characteristics associated with volcanic environments, the bulk resistivity properties of rocks in general need to be considered in the 
interpretation of this thesis.

\subsection{Bulk Resistivity of Rocks}

The bulk electrical resistivity of a rock formation is an average value dependent on:

- The individual grain types that make up the rock.

- The porosity of the rock.

- The amount and type of any fluids that are present.

- The connectivity of any fluids.

- The degree and type of any alteration that has occurred.

- The temperature.

There is often a mixture of rock types within a layer (e.g. pyroclastic material and lava), the conductivity of the mixture of two materials is given by the Hashin-Shtrikman limits (Hashin and Shtrikman, 1963).

$$
\begin{gathered}
\sigma_{\text {upper }}=\frac{3 \sigma_{2}-2 f\left(\sigma_{2}-\sigma_{1}\right)}{3 \sigma_{1}+f\left(\sigma_{2}-\sigma_{1}\right)} \sigma_{1} \\
\sigma_{\text {lower }}=\frac{2 \sigma_{2}+\sigma_{1}+2 f\left(\sigma_{1}-\sigma_{2}\right)}{2 \sigma_{2}+\sigma_{1}-f\left(\sigma_{1}-\sigma_{2}\right)} \sigma_{2}
\end{gathered}
$$

where $\sigma_{1}$ and $\sigma_{2}$ are the conductivity of each material separately, with $\sigma_{1}$ being the better conductor and $f$ being the volume fraction of this better conductor. The actual conductivity value will be between these two limits.

Generally when a rock contains fissures, fractures, or pores filled with a lower resistivity material such as water, the overall resistivity of the rock will be greatly reduced as electricity prefers to travel along the path of least resistance. If rocks are at high temperatures or the fluids are saline then the resistivity recorded is even lower. Archie's Law empirically describes this relationship as

$$
\sigma=\zeta \sigma_{w} P^{2}
$$


where $\zeta$ is the degree of saturation, $\sigma_{w}$ is the conductivity of the fluid and $P$ is the porosity of the rock. The above equations can be used to constrain some of the fluid properties within a lithology.

Previous MT studies have identified the electrical properties of many similar lithologies within New Zealand. In the TVZ ignimbrites have had measured resistivity values between 200 and $500 \Omega m$ (Bibby et al., 1998). Tertiary sediment in the Hikurangi forearc has a measured resistivity of $<10 \Omega m$ (Ingham et al., 2001). Resistivities of the order of a few hundred to a few thousand $\Omega m$ have been attributed to the presence of greywacke basement (Ingham et al., 2001; Bibby et al., 1998). These values are expected to be representative of these lithologies within the TVC as well. 
CHAPTER 3

\section{THEORY}

Magnetotellurics (MT) is a geophysical technique that takes advantage of the natural variations of the external geomagnetic field and in which the induced electric field response within the Earth is measured. The relationship between the electric and magnetic fields is a function of the nearby conductivity structure. This chapter explains how the relationship between the fields is determined. In addition the effects and removal of distorters, and the theory behind the processing and modelling of data are discussed. All variables used in this chapter are defined in Appendix D.

\subsection{Electromagnetic Induction within the Earth}

The theory of EM induction is derived from Maxwell's equations. In terms of the magnetic field $(\underline{B})$ and electric field $(\underline{E})$, these equations are written as:

$$
\begin{gathered}
\nabla \times \underline{B}=\mu_{0}\left(\underline{j}+\epsilon_{0} \frac{\partial \underline{E}}{\partial t}\right) \\
\nabla \times \underline{E}=-\frac{\partial \underline{B}}{\partial t} \\
\nabla \cdot \underline{E}=\frac{q}{\epsilon_{0}} \\
\nabla \cdot \underline{B}=0
\end{gathered}
$$

When equation 3.1 is manipulated in conjunction with Ohm's Law $(\underline{j}=\sigma \underline{E}), \underline{E}$ 
can be expressed as a function of $\underline{B}$.

$$
\nabla \times \underline{B}=\mu_{0}\left(\sigma \underline{E}+\epsilon_{0} \frac{\partial \underline{E}}{\partial t}\right)
$$

As $\epsilon_{0} \approx 8 \times 10^{-12} \mathrm{~F} / \mathrm{m}$ and $\mu_{0} \approx 4 \pi \times 10^{-7} \mathrm{H} / \mathrm{m}$, when this equation is solved at typical crustal values the second term on the right (the current displacement term) can be ignored for all frequencies less then $10^{-4} \mathrm{~Hz}$. Thus for our purpose this equation simplifies to

$$
\nabla \times \underline{B}=\mu_{0} \sigma \underline{E}
$$

Furthermore $\underline{E}$ can be eliminated from equation 3.5 by applying equation 3.2 and using the algebraic identities below.

$$
\begin{gathered}
\nabla \times(a \underline{U})=a(\nabla \times \underline{U}) \\
\nabla \times(\nabla \times \underline{U})=\nabla \cdot(\nabla \cdot \underline{U})-\nabla^{2} \underline{U}
\end{gathered}
$$

This results in the diffusion equation for a time varying magnetic field.

$$
\nabla^{2} \underline{B}=\mu_{0} \sigma \frac{\partial \underline{B}}{\partial t}=-i \omega \mu_{0} \sigma \underline{B}=k^{2} \underline{B}
$$

where a time variation of the form $e^{-i \omega t}$ has been assumed. Deducing the time varying electric field diffusion equation is achieved through a similar manipulation of Maxwell's equations giving,

$$
\nabla^{2} \underline{E}=\mu_{0} \sigma \frac{\partial \underline{E}}{\partial t}+\nabla\left(\frac{q}{\epsilon_{0}}\right)
$$

The additional term in equation 3.7 is due to electrical diffusion causing charge accumulation on any boundary. When applying the theory to modelling MT data in 2 or 3-dimensions this term is accommodated in the boundary conditions.

As with many physical fields measured in the time domain, the mathematics is less complicated in the Frequency domain. Applying a Fourier transform easily converts between the domains, thus both fields have a time dependent component, expressed in the form of $e^{-i \omega t}$ in the frequency domain. 
$\underline{B}$ can be treated separately for each frequency as equation 3.6 is linear. The frequency and ambient electrical conductivity of a medium will determine the depth of penetration for each wave form within $\underline{B}$. If $\underline{B}$ is considered to be a horizontally oscillating field in the plane of a semi-infinite conductor, then the general waveform in the medium is:

$$
\underline{B}=\underline{B}_{0} e^{\alpha z} e^{-i \omega t}
$$

where $z$ is positive downwards.

Substituting equation 3.8 into the diffusion equation means the value of $\alpha$ is:

$$
\alpha= \pm(1-i) \sqrt{\frac{\mu_{0} \sigma \omega}{2}}
$$

Physically $\underline{B}$ must decrease with depth and therefore only the negative solution is applicable. The distance required for the amplitude of the wave to decrease by a factor of $1 / e$ is defined to be the skin depth $(\delta)$, which can be regarded as the effective penetration depth for variations of a particular frequency.

$$
\delta=\sqrt{\frac{2}{\mu_{0} \sigma \omega}}
$$

The general waveform (3.8) can be defined more specifically as

$$
\underline{B}=\underline{B}_{0} e^{\frac{-z}{\delta}} e^{-i\left(\omega t-\frac{z}{\delta}\right)}
$$

Similarly the induced field $(\underline{E})$ can be shown to be frequency dependent by substituting the magnetic waveform above into equation 3.2.

$$
\nabla \times \underline{E}=i \omega \underline{B}
$$

This gives a frequency dependent phase shift between the magnetic field and the induced electric field.

When constraints such as dimensionality are applied to the situation, these equations can be simplified to identify features within the Earth. 


\subsubsection{1-Dimensional Earth Solutions}

When the Earth is considered to be a set of concentric homogeneous spheres, then for any point on the surface of the Earth the conductivity structure only varies in a radial direction. In this 1-D situation the conductivity of the Earth can be analytically resolved from the MT sounding.

The analytical solution is derived from the knowledge that the EM field obeys Laplace's equation, $\nabla^{2} \Psi=0$, at the surface (here $\Psi$ is any function of $\theta, \phi$ and $r$ ). Laplace's equation is solved by equating the negative gradient of the external field to the divergence of the internal field.

As before the magnetic field is assumed to have a time dependence of $e^{-i \omega t}$ in the frequency domain and must satisfy the diffusion equation.

$$
\nabla^{2} \underline{B}=k^{2} \underline{B}
$$

Outside a uniform sphere the external magnetic field $\underline{U}_{e}$ has a potential of the form

$$
\underline{U}_{e}=B_{e} \frac{r^{n}}{a^{n-1}} S_{n}^{m}(\theta, \phi) e^{-i \omega t}
$$

where $S_{n}^{m}(\theta, \phi)$ is a surface harmonic of the order $n$ and degree $m$. Inside this uniform sphere the internal magnetic field may be described by the sum of poloidal and toroidal fields. These are the magnetic fields that are generated inside the earth. The toroidal field has no radial component and cannot be detected outside the sphere. Consequently its divergence is zero and the toroidal field does not contribute to the internal magnetic field at the surface. The internal field can thus be represented by the equations

$$
\begin{aligned}
B_{r} & =C \frac{F}{r} n(n+1) S_{n} \\
B_{\theta} & =\frac{C}{r} \frac{\partial(r F)}{\partial r} \frac{\partial S_{n}}{\partial \theta} \\
B_{\phi} & =C \frac{1}{r \sin \theta} \frac{\partial(n F)}{\partial r} \frac{\partial S_{n}}{\partial \theta}
\end{aligned}
$$

where $F$ is an arbitrary function, and $m$ in the spherical harmonic term and the longitudinal dependence $(\phi)$ are dropped due to symmetry. When the diffusion equation 
(3.12) is solved, $F$ has the form of a Bessel function. As $F$ can not become infinite as $r$ approaches zero, the negative order functions have no significance within a uniform Earth, meaning the range of possible solutions is halved. However in a 1-D Earth consisting of concentric shells of differing conductivity, the negative order functions are used to solve for boundary conditions between each shell.

When considering frequencies higher than the diurnal variation such as those used in an MT sounding, this process is simplified by ignoring the sphericity of the Earth. The surface conditions are evaluated through planar geometrical consideration of a uniform half space. Under these conditions the external field $(U)$ can be represented by the sum of a series of elliptically polarized waves travelling in a horizontal direction at a velocity of $(\omega / v)$.

In planar geometry the magnetic fields are more simply described. The first step in calculating the conductivity at the surface of the Earth is to equate the external and internal magnetic fields. Outside the Earth the magnetic field is described by $\underline{B}=-\nabla \underline{U}$, while inside the Earth it is described as $\underline{B}=\nabla \times \underline{A}$ where A is a magnetic vector potential. As these are equated at the surface where $z=0$.

$$
-\nabla \underline{U}=\nabla \times \underline{A}
$$

The magnetic potentials $\underline{A}$ and $\underline{U}$ have the forms:

$$
\begin{gathered}
\underline{U}(y, z)=\frac{-1}{v} e^{i v y}\left(B_{e} e^{-v z}+B_{i} e^{v z}\right) e^{-i \omega t} \\
\underline{A}=A_{x}=F(y)\left(C e^{-\alpha z}+D e^{\alpha z}\right) e^{-i \omega t}
\end{gathered}
$$

When solving the diffusion equation for the internal field as required by equation 3.6, a second order differential equation with respect to $F(y)$ is obtained.

$$
\frac{\partial^{2} F(y)}{\partial y^{2}}+\left(\alpha^{2}+i \omega \mu_{0} \sigma\right) F(y)=0
$$

Under the assumption that $F(y)$ has an exponential form, the differential equation can 
be solved by

$$
F(y)=e^{i\left(\alpha^{2}-k^{2}\right)^{\frac{1}{2}} y}
$$

Additionally $A_{x}$ must become zero as $z$ becomes infinity, consequently $D=0$ in equation 3.17. The boundary conditions at the Earth's surface $(z=0)$ require that the tangential components of the magnetic field are continuous across the boundary. Equating the y-components

$$
i e^{i v y}\left(B_{e}+B_{i}\right)=F(y)(-\alpha C)
$$

As the dependency on $y$ of each side must be equal in order for the equality to hold, this implies that $F(y)=e^{i v y}$. Comparing to equation 3.18 this leads to the conclusion that

$$
\alpha^{2}=v^{2}-k^{2}
$$

Equating the remaining components shows that

$$
B_{e}-B_{i}=i v C
$$

When equation 3.20 is solved simultaneously with equation 3.19 , the ratio of $B_{i}: B_{e}$ is obtained

$$
\frac{B_{i}}{B_{e}}=\frac{\alpha-v}{\alpha+v}
$$

Equations $3.15-3.21$ lead to a situation where it is possible to determine the ratios of $B_{z} / B_{y}$ and $E_{x} / B_{y}$, which are ratios measurable in the field at the surface where $z=0$. The components $B_{z}$ and $B_{y}$ are calculated from the external field (equations 3.15 and 3.16 ), and simplify to result in

$$
\frac{B_{z}}{B_{y}}=\frac{i v}{\left(v^{2}+k^{2}\right)^{\frac{1}{2}}}
$$

This implies that under the assumption of a plane wave with $v=0$, there is no variation in the $B_{z}$ component of the external EM field.

Through equations 3.2 and 3.15, it can be shown that the gradient of a function 
$\Psi$ equals $\underline{E}+\frac{-\partial \underline{A}}{\partial t}$, but as $E_{x}$ is parallel to the surface and no charge accumulates the function must equal zero therefore

$$
\underline{E}=\frac{-\partial \underline{A}}{\partial t}
$$

From this the ratio

$$
\frac{E_{x}}{B_{y}}=\frac{-i \omega}{\left(v^{2}+k^{2}\right)^{\frac{1}{2}}}
$$

can be calculated. These equations are generalized for the surface, but can be extended to a series of layer interfaces in order to calculate the EM field ratio at each interface, leading to the expected ratio of $E_{x}$ to $B_{y}$ over a general 1-D Earth.

\subsubsection{2 and 3-dimensional Earth solutions}

Analytical solutions can only be derived for a 1-D Earth. 2 and 3-D solutions are numerical. In a 2-D situation it is generally assumed that the invariant direction is labelled $x$. From Maxwell's equations (3.1 to 3.4) and continuing the assumptions of negligible displacement currents, and an $e^{-i \omega t}$ time dependence, the 2-D numerical solution is found through the following electric and magnetic field relations:

$$
\nabla \times \underline{B}=\mu_{0} \sigma(y, z) \underline{E}
$$

and

$$
\nabla \times \underline{E}=i \omega \underline{B}
$$

These equations can be broken down into six component equations.

$$
\begin{gathered}
\frac{\partial B_{z}}{\partial y}-\frac{\partial B_{y}}{\partial z}=\mu_{0} \sigma E_{x} \\
\frac{\partial B_{x}}{\partial z}=\mu_{0} \sigma E_{y} \\
-\frac{\partial B_{x}}{\partial y}=\mu_{0} \sigma E_{z} \\
\frac{\partial E_{z}}{\partial y}-\frac{\partial E_{y}}{\partial z}=i \omega B_{x}
\end{gathered}
$$




$$
\begin{gathered}
\frac{\partial E_{x}}{\partial z}=i \omega B_{y} \\
-\frac{\partial E_{x}}{\partial y}=i \omega B_{z}
\end{gathered}
$$

These equations form two sets, representing the E-polarization and the B-polarization solutions. Equations 3.26, 3.30, and 3.31 are the E-polarization set and are solely comprised of the variables $E_{x}, B_{y}$, and $B_{z}$; these equations are combined to form an expression involving only $E_{x}$.

$$
\frac{\partial^{2} E_{x}}{\partial y^{2}}+\frac{\partial^{2} E_{x}}{\partial z^{2}}=-i \omega \mu_{0} \sigma E_{x}
$$

Similarly equations 3.27, 3.28 and 3.29 are the B-polarization set, comprised of variables $B_{x}, E_{y}$ and $E_{z}$; thus producing the $B_{x}$ function.

$$
\frac{\partial^{2} B_{x}}{\partial y^{2}}+\frac{\partial^{2} B_{x}}{\partial z^{2}}+\frac{1}{\sigma}\left(\frac{\partial \sigma}{\partial y} \frac{\partial B_{x}}{\partial y}+\frac{\partial \sigma}{\partial z} \frac{\partial B_{x}}{\partial z}\right)=-i \omega \mu_{0} \sigma B_{x}
$$

These equations still require numerical solutions for specified conductivity structures. If the structure is grided into a series of boxes of constant conductivity, equation 3.33 can then be simplified to the form of equation 3.32. These equations are then solved with respect to the following set of boundary conditions.

\section{Boundary Conditions:}

1. The first boundary condition that must be satisfied is determined by applying equation 3.4 to a small cylinder encompassing the boundary (see figure 3.1 (a)). For this cylinder

$$
\int \nabla \cdot \underline{B} d V=\int \underline{B} \cdot n d \underline{S}=0
$$

In order to satisfy this condition, when the height of the cylinder is small, the normal component of $\underline{B}$ must be continuous across the boundary. Therefore

$$
\left(B_{n}\right)_{1}=\left(B_{n}\right)_{2}
$$

where $_{1}$ and ${ }_{2}$ refer to the media on either side of the boundary. 


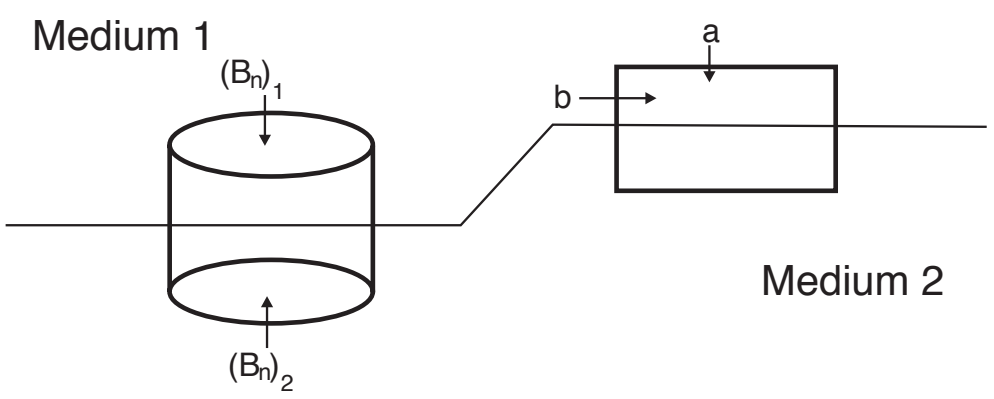

(a)

(b)

Figure 3.1 Simple figure used in the development of boundary conditions in MT theory. (a) small cylinder enclosing the boundary as used in determining condition 1; (b) rectangle enclosing boundary as used in determining conditions 2 and 3.

2. The second boundary condition is obtained from integrating around a rectangle enclosing the boundary (see figure 3.1 (b)).

$$
\int \underline{B} \cdot \underline{d l}=\left\{\left(B_{t}\right)_{1}-\left(B_{t}\right)_{2}\right\} a
$$

Applying equation 3.1 and taking the limit as the rectangle becomes a line, the relationship is reduced to

$$
\left(B_{t}\right)_{1}-\left(B_{t}\right)_{2}=\mu_{0} j_{s}
$$

where $j_{s}$ is the surface current at the boundary.

3. The third condition arises from applying equation 3.2 to the same rectangle as in boundary condition 2. This leads to,

$$
\int \underline{E} \cdot \underline{d l}=\int(\nabla \times \underline{E}) \cdot \underline{d S}=-\int \frac{\partial \underline{B}}{\partial \underline{t}} \cdot n \underline{d S}
$$

When the limits are taken as the rectangle becomes the boundary, this simplifies to the condition that the tangential component of $\underline{E}$ must be continuous across the boundary.

$$
\left(E_{t}\right)_{1}=\left(E_{t}\right)_{2}
$$

4. The fourth boundary condition is from the observation that there is no surface charge on the boundary. This implies that the normal component of the current 
density must be continuous across the boundary, thus

$$
\left(j_{n}\right)_{1}=\left(j_{n}\right)_{2}
$$

The proposed conductivity structure response is derived by, for example, representing equations 3.32 and 3.33 in finite difference formulations for each constant conductivity box and then matching the four boundary conditions on all boundaries. Comparison of the model response at the surface is then made with the observed data to determine the appropriateness of the proposed structure. The conductivity structure is then altered and the response re-calculated until a reasonable fit to the observed data is obtained.

In 3-D the conductivity structure is resolved in much the same manner, expect there is no simplification of Maxwell's equations and the boundary conditions must now apply to all six faces of a conductivity cube. Instead of solving for $B$ and E-polarization sets, the structure is solved for the single equation

$$
\mu_{0} \sigma \nabla^{2} \underline{B}+\nabla \mu_{0} \sigma \times(\nabla \times \underline{B})-\mu_{0}^{2} \sigma^{2} \frac{\partial \underline{B}}{\partial t}=0
$$

Various authors have used perturbations of lower dimensional structures to make up a 3-D structure. The computing power required to solve all the simultaneous equations required for a 3-D structure had been prohibitive in many studies, although recent advances in technology are eliminating this issue.

\subsection{Magnetotelluric Soundings}

An MT sounding uses the principle of measuring the natural variations in the Earth's electric and magnetic fields to determine local structure around the station from the relationship between the measured fields. For each field, two horizontally orthogonal components of the field are recorded simultaneously. The vertical component of the magnetic field is also recorded.

The equations are simplified if the horizontal components are aligned parallel and 
perpendicular to any existing 2-D strike, in which case the measured fields correspond to the $E$ and $B$-polarization fields. Alternatively the coordinate axes can be mathematically rotated during processing. It is standard procedure to align the station so that one horizontal component is oriented magnetic north/south and the other magnetic east/west and rotate the data as necessary during processing. The components are identified as

- $N$ - the north/south component of $\underline{E}$

- $E$ - the east/west component of $\underline{E}$

- $H$ - the north/south component of $\underline{H}$

- $D$ - the east/west component of $\underline{H}$

Where $\underline{H}$ is related to $\underline{B}$ by

$$
\underline{H}=\mu_{0} \underline{B}
$$

The resistivity of free air is much greater (effectively 'infinite') than that of the solid Earth, consequently all electric fields and currents are considered to be horizontal at the surface of the Earth. Under these conditions the relationship between the components gathered from each site may be represented in the time domain by

$$
N^{\prime}(\tau)=\int Z_{1}(t-\tau) H^{\prime}(t) d t+\int Z_{2}(t-\tau) D^{\prime}(t) d t
$$

and

$$
E^{\prime}(\tau)=\int Z_{3}(t-\tau) H^{\prime}(t) d t+\int Z_{4}(t-\tau) D^{\prime}(t) d t
$$

Here $Z_{i}$ represents the Earth's impulse response to components of the inducing horizontal magnetic field. The integrals therefore represent the convolution of the magnetic field with the impulse response of the Earth. Applying a Fourier transform to the time series data converts the relationship into the frequency domain, where it is more simply described as

$$
\underline{E}=\underline{\underline{Z H}}
$$


where $\underline{\underline{Z}}$ is the impedance tensor. Equation 3.41 breaks down into component parts such that

$$
N=Z_{x x} H+Z_{x y} D
$$

and

$$
E=Z_{y x} H+Z_{y y} D
$$

where $Z_{x x}, Z_{x y}, Z_{y x}$ and $Z_{y y}$ are the Fourier transforms of the components $Z_{1}, Z_{2}, Z_{3}$, and $Z_{4}$ in equations 3.39 and 3.40 , and $N, E, H$, and $D$ are Fourier transforms of the measured fields.

When the conductivity structure beneath a site is strictly 1-D, the magnetic field is solely related to its orthogonal electric component. In this case

- an eastward magnetic field induces a northward electric field,

- and a northward magnetic field induces a westward electric field.

Therefore both $Z_{x x}$ and $Z_{y y}$ are zero, and as there is no preferred horizontal direction,

$$
Z_{x y}=-Z_{y x}
$$

The impedance tensor elements $Z_{i j}$ are complex and therefore it is possible to express each element in terms of an apparent resistivity $\left(\rho_{a}\right)$ and a phase $(\Phi)$ at each frequency.

$$
\begin{gathered}
\rho_{a}=\frac{1}{\mu_{0} \omega}\left|Z_{i j}\right|^{2} \\
\Phi_{a}=\tan ^{-1} \frac{\Im(Z)}{\Re(Z 0)}
\end{gathered}
$$

Where $\Re(Z)$ and $\Im(Z)$ are the real and imaginary parts of $Z_{i j}$ respectively.

From the skin depth equation (3.9) it is observed that as the period increases so too does the skin depth. Therefore if the apparent resistivity is plotted against the period, the plot is analogous to a resistivity versus depth plot. When more than one site along a traverse has been occupied, it is common to create a psuedosection with horizontal distance between the sites along the top, the logarithm of the period on the vertical, 
and the apparent resistivity colour contoured as a 'cross section'. Alternatively the phase variation is plotted as an indication of how quickly the resistivity below a site is changing. A phase value of $45^{\circ}$ is recorded when the resistivity structure is uniform; as the resistivity increases the phase decreases and vice versa.

When the structure is 2-D, all four of the impedance tensor elements are in general non-zero, unless the impedance tensor is rotated into the strike direction in which case $Z_{x x}$ and $Z_{y y}$ again become zero. In this situation $Z_{x y} \neq Z_{y x}$, one is aligned parallel to strike direction $\left(Z_{\|}\right)$and the other is aligned perpendicular to the strike $\left(Z_{\perp}\right)$ corresponding to the $E$ and $B$-polarisation modes. In practice the E-polarisation is known as the TE mode and the B-polarisation as the TM mode.

If the strike of the 2-D structure is unknown it maybe estimated by finding the orientation of axes for which $Z_{x x}$ and $Z_{y y}$ are minimum. This is equivalent to maximizing $\left(\left|Z_{x y}^{\prime}\right|^{2}+\left|Z_{y x}^{\prime}\right|^{2}\right)$. The estimated strike angle is then given by

$$
\theta=\frac{1}{4} \tan ^{-1} \frac{\left(Z_{x x}-Z_{y y}\right)\left(Z_{x y}+Z_{y x}\right)^{*}+\left(Z_{x x}-Z_{y y}\right)^{*}\left(Z_{x y}+Z_{y x}\right)}{\left|Z_{x x}-Z_{y y}\right|^{2}-\left|Z_{x y}+Z_{y x}\right|^{2}}
$$

There is a $\pi / 2$ ambiguity in the solution of this equation, consequently it is not possible to determine if $Z_{\|}$is equivalent to the $Z_{x y}$ or $Z_{y x}$ component without additional information (Swift, 1967, cited in Ingham (1988)). In general on the resistive side of a vertical boundary $Z_{\perp}$ is larger than $Z_{\|}$, therefore with prior knowledge $Z_{\|}$and $Z_{\perp}$ can be correctly identified.

Once the strike direction is calculated, the rotation matrix $\underline{\underline{R}}$ rotates the impedance tensor to obtain the TE and TM modes.

$$
\underline{\underline{R}}=\left[\begin{array}{cc}
\cos \theta & \sin \theta \\
-\sin \theta & \cos \theta
\end{array}\right]
$$


From equation 3.41 it can be seen that

$$
\begin{aligned}
\underline{E}^{\prime} & =\underline{\underline{R E}} \\
& =\underline{\underline{R Z}} \\
& =\underline{\underline{R Z R^{-1}}}{\underline{H^{\prime}}}^{\prime} \\
& =\underline{\underline{Z^{\prime} H^{\prime}}}
\end{aligned}
$$

Where $\underline{\underline{Z}}^{\prime}=\underline{R Z R}^{-1}$. Therefore, the rotation is directly applied to the impedance tensor.

When the structure is 3-D all elements of the impedance tensor are non-zero regardless of the orientation of the measurement system. Generally in this case there are ranges of periods for which the structure behaves in a 2-D manner and over which the data may be modelled as such. However when dealing with a 3-D impedance tensor the common assumption of a 3-D heterogeneity overlaying a 2-D regional structure is often applied (Groom and Bailey, 1989). In this case, at long periods the heterogeneity is often assumed to cause a galvanic distortion (one that is invariant with period), and the impedance can be decomposed to a 2-D tensor by removing the effects of the galvanic distortion (refer to section 3.3.2).

The determinant is a single value that can be used to represent the entire impedance tensor regardless of dimensionality. These "effective impedances" (Berdichevsky and Dmitriev, 1976, cited in Jones (1988)) are co-ordinate invariant and can determine an average 1-D structure beneath the site. The determinant impedance is given by

$$
Z_{\text {det }}=\sqrt{Z_{x x} Z_{y y}-Z_{x y} Z_{y x}}
$$

No assumptions have been made on the conductivity structure in calculating the determinant in equation 3.49 so this invariant can be applied to data of any dimensionality and is often used to determine dominant structural features.

The dimensionality of the impedance tensor is often determined so that the best processing and modelling techniques can be applied. The most common way to determine the dimensionality is to calculate the skew (Swift, 1967, cited in Ingham (1988)), 
which is independent of the orientation of the measurement system.

$$
S=\left|\frac{Z_{x x}+Z_{y y}}{Z_{x y}-Z_{y x}}\right|
$$

In a 1-D structure both $Z_{x x}$ and $Z_{y y}$ are zero so $S=0$. This is again the case in a 2-D structure but there is considerable difference between $Z_{x y}$ and $Z_{y x}$. In a 3-D structure $S \neq 0$ under any circumstances. Another method sometimes used is that of Kao and Orr (1982) that assigns a degree of each dimensionality to the data. This allows direct measurement of the importance of different structural components within the Earth.

Generally the skew is calculated early in the processing procedure to determine the best way to process the data. An inherent limitation of the MT method is the loss of resolution with depth. In addition to dimensionality altering the appearance of the impedance tensor, so too does the presence of any distortion. Distortion needs to be removed from the data before any modelling takes place if a realistic structure is to be obtained.

\subsection{Distortion}

Distortion is often split into inductive and galvanic effects. The inductive effects are caused by topographical variations and by conductivity anomalies of relatively large scale, whereas the galvanic effects are caused by charge accumulation on small scale inhomogeneities within the structure. In the frequency range of interest in MT studies, galvanic distortion is generally regarded as only effecting the electric field. In contrast inductive effects occur in both $\underline{E}$ and $\underline{B}$, and may be regarded as imposing true 3dimensionality on the data. Galvanic distortion removal has been the subject of a considerable amount of MT literature (e.g. Caldwell et al. (2004), Weaver et al. (2000), Lilley (1998), Bahr (1991), and Groom and Bailey (1989; 1991)). In this section the effects of topography are discussed, followed by the most commonly used method of galvanic distortion removal prior to an explanation of the new distortion removal method being applied in this study. 


\subsubsection{Topography}

Topography causes an inductive effect that only influence high frequency data (Jiracek, 1990), although the effects of large topographical features are seen at lower frequencies. Induction is due to the 'infinite' resistivity of free air around a topographical change. Along a ridge system there will be a increase in the electric field strength-due to electricity preferring to travel through the lower resistivity rock. However, across a topographical feature the same amount of energy is contained in the electric field and this energy is either stretched or compressed to fill the space within the rock, resulting in a decreased field strength on a ridge and increased field strength in a valley.

The remote reference method (Gamble et al., 1979) used to remove local noise cannot decrease the noise as effectively in areas with large topographical changes. The amplitude, extent of noise, and the variability of the magnetic field are all higher in mountainous areas (Ádám et al., 1986). So although a remote reference is used, topographical effects cannot be completely removed from data.

In general, when large scale topography is involved in a survey area then it is included in the model for realistic interpretation of the data. This has been done on volcanos in 2-D surveys (Fuji-ta et al., 1999; Ogawa et al., 1998) and more recently in 3D (Müller and Haak, 2004). As most models use a cubic griding system to represent the conductivity structure, sites on the sides of volcanoes have to be considered carefully. Both Fuji-ta et al. (1999) and Ogawa et al. (1998) created their models as if there was no topography, subsequently transforming the grid squares into parallelograms to represent the surface topography.

Alternatively Müller and Haak (2004) retained the grid squares, but had the surface in different rows to represent the topography. At very high frequencies the inclusion of topography in the modelling unnecessarily overcomplicates the model in a survey of this size. The new phase tensor analysis (see section 3.3.5) provides a way to identify where the data is being effected by inductive distortion. 


\subsubsection{Galvanic Distortion}

Of more concern during the analysis of MT data are the effects of galvanic distortion. This is caused by "galvanic charge formed at the boundaries of a near surface conductivity heterogeneity by the flux of regional current through the heterogeneity" (Jiracek, 1990).

Galvanic distortion can be represented by a real matrix $\underline{\underline{C}}$ that is independent of frequency. $\underline{\underline{C}}$ acts on the impedance tensor so that the measured impedance tensor $\underline{\underline{Z}}^{\prime}$ becomes

$$
\underline{\underline{Z}}^{\prime}=\underline{\underline{C Z}} R
$$

where $\underline{\underline{Z}}^{\prime}$ is a complex matrix of the form

$$
\underline{\underline{Z}}^{\prime}=\left[\begin{array}{ll}
Z_{11} & Z_{12} \\
Z_{21} & Z_{22}
\end{array}\right]
$$

and $\underline{\underline{C}}$ is a real matrix of the form

$$
\underline{\underline{C}}=\left[\begin{array}{ll}
a & b \\
c & d
\end{array}\right]
$$

When 2-D data are affected by galvanic distortion a rotation of the impedance tensor $\underline{\underline{Z}}^{\prime}$ as in section 3.1 .2 will not provide an accurate estimate of the strike direction. Thus when galvanic distortion is present a method of removal of the distortion is required prior to analysing the data. The first step is often the removal of the static shift.

\subsubsection{Static shift}

Physically the cause of static shift is an inhomogeneity (e.g. a boulder) situated close to one of the measuring electrodes. The inhomogeneity will either gather or deflect the electric current passing through the ground. If the inhomogeneity has a lower resistivity than the surrounding material, the electric currents will be attracted to the inhomogeneity and a lower apparent resistivity value will be recorded at the surface. Whereas if the resistivity is greater than the surrounding material, electric currents 
will bend away from the inhomogeneity, thus increasing the magnitude of the recorded electric field. This affect was best illustrated by Jiracek (1990), and is reproduced here as figure 3.2 .

\section{Total Current}
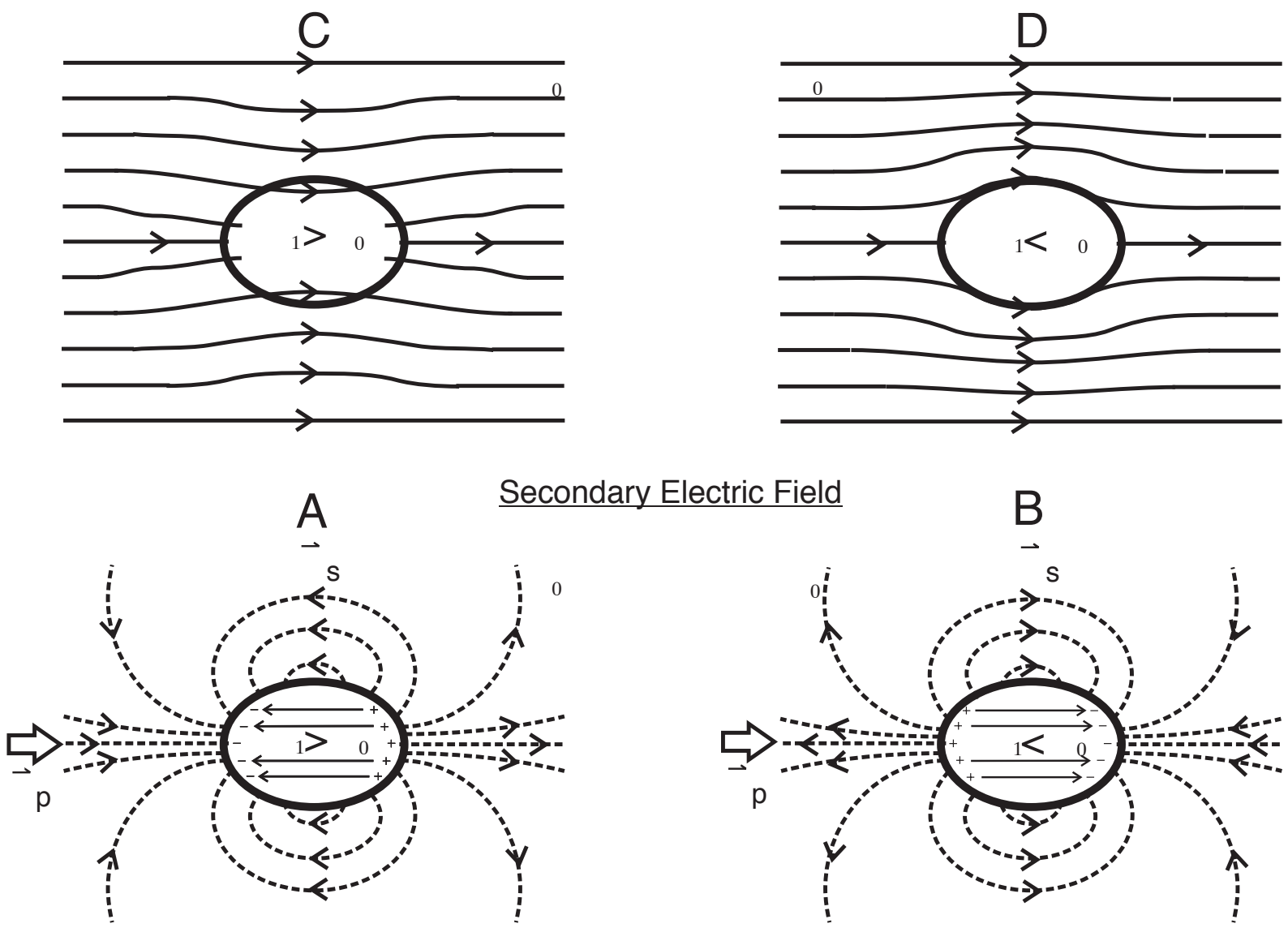

Figure 3.2 The effects of inhomogenieties on electric fields. The secondary electric fields being created by: (A), a conductive boulder, (B), a resistive boulder, and their effect on the total current, $(C)$, and $(D)$ respectively. Picture taken from Jiracek (1990)

Static shift can be regarded as a special case of galvanic distortion, and although the magnitudes of the apparent resistivities are changed, the phases are unaffected. This has the effect of multiplying each impedance tensor element independently by a constant value. When the apparent resistivity is calculated a constant real component $(g)$ is extracted such that, for an impedance tensor that is in reality 2-D

$$
\underline{\underline{Z}}=\left[\begin{array}{cc}
0 & Z_{x y} \\
Z_{y x} & 0
\end{array}\right]
$$




$$
\begin{aligned}
\rho_{a 2} & =\frac{1}{\mu_{0} \omega}\left|g Z_{x y}\right|^{2} \\
& =\frac{1}{\mu_{0} \omega} g^{2}\left|Z_{x y}\right|^{2}
\end{aligned}
$$

When displayed on a log scale the apparently resistivity is simply shifted vertically

$$
\begin{aligned}
\log \rho_{a 2} & =\log g^{2}+\log \frac{1}{\mu_{0} \omega}\left|Z_{x y}\right|^{2} \\
& =\log g^{2}+\log \rho_{a}
\end{aligned}
$$

Consequently there is no effect on the shape of the resistivity curve when plotted against the period, the resistivities are simply shifted up or down the vertical axes by a value dependent on $g$.

Whether static shift has effected data cannot be determined unless there is other information available. There are five ways to acquire this additional information in order to correct for static shift:

1. If more than one site has been occupied in the area, the curves can be shifted until they match at a particular frequency.

2. A controlled source EM survey can be conducted and the true apparent resistivity calculated from the magnetic data (which are unaffected by static shift) (Fluche, 1983).

3. An accepted regional apparent resistivity curve may be used and the data shifted vertically until a match is achieved with the regional curve.

4. A parametric method may be used that assumes an underlying layer of uniform resistivity. Each curve is then shifted to match that layer (Jones, 1988).

5. A DC resistivity sounding may be made and used to estimate the true near surface apparent resistivity (McLaughin et al., 2002).

In situations where the distortion is more complicated, a static shift correction will be insufficient to obtain the regional impedance tensor. To date the Groom and Bailey decomposition method is the most popular for the removal of additional galvanic effects. 


\subsubsection{The Groom and Bailey Decomposition Method}

Groom and Bailey (1989) developed a general method that allows for a number of galvanic effects to be removed from the impedance tensor, although numerous updates have been added over the years (Groom and Bailey, 1991; Bahr, 1991). The underlying assumption required for this method to work is that while the distortion is $3-\mathrm{D}$, the underlying regional structure is only 2-D.

The distortion tensor $(\underline{\underline{C}})$ can be split into three tensors and a scaler in the GroomBailey analysis, each used to represent one of the four distortion sources.

$$
\underline{\underline{C}}=g \underline{\underline{A T S}}
$$

Each of these sources are described below:

- $\underline{\underline{A}}$ allows splitting of the electric field, this introduces anisotropy into the impedance tensor.

$$
\underline{\underline{A}}=\left[\begin{array}{cc}
1+s & 0 \\
0 & 1-s
\end{array}\right]
$$

- $\underline{\underline{T}}$ allows twisting of the electric field.

$$
\underline{\underline{T}}=\left[\begin{array}{cc}
1 & -t \\
t & 1
\end{array}\right]
$$

- $\underline{\underline{S}}$ allows shearing of the electric field.

$$
\underline{\underline{S}}=\left[\begin{array}{ll}
1 & e \\
e & 1
\end{array}\right]
$$

- $g$ is the static shift scaler described in section 3.3.3 above.

As galvanic distortion is assumed to only effect the electric field, it is assumed that $\underline{\underline{B}}$ is unaltered. Therefore all the above tensors can be applied directly to the impedance tensor. If rotation is also taken into consideration then the observed impedance tensor 
$\left(\underline{\underline{Z}}^{\prime}\right)$ is related to the regional impedance tensor $\left(\underline{\underline{Z}}_{R}\right)$ by

$$
\underline{\underline{Z}}^{\prime}=\underline{\underline{R}} g \underline{\underline{A T S Z}} R \underline{\underline{R}}^{-1}
$$

As $\underline{\underline{Z}}^{\prime}$ has complex components, this relationship has eight equations with nine unknown parameters (the real and imaginary parts of the off-diagonal elements of the regional impedance tensor, $s, t, e, g$ and $\theta$ the rotation angle from the regional strike direction) when $\underline{\underline{Z}}_{R}$ is assumed to be 2-D. Thus the equations are underdetermined. Assuming that $e, t$ and $\theta$ are all independent of frequency, yields an over determined set of equations requiring a numerical fit to minimize the errors. Generally after running a number of decompositions with combinations of these parameters held constant, a final regional impedance tensor is derived from the best fitting solutions with all three parameters constrained.

An alternative method was proposed by Lilley (1998) (and elaborated on by Weaver et al. (2000)), based on the Mohr circles of structural geology. This plots the variations with axial rotations of the real and imaginary parts of the impedance tensor separately on a graph. This method uses the same assumptions concerning underlying dimensionality but is conducted through the drawing of graphs as opposed to the mathematical approach of Groom and Bailey. The recently introduced phase tensor method allows the dimensionality to be undefined during the distortion removal process.

\subsubsection{The Phase Tensor Method}

The phase tensor method allows the distortion to be determined using only the phase components of a MT data set and was developed by Caldwell et al. (2004). Previously the idea of using just the phase to interpret data has been identified and used by Parks et al. (1988), and Mozley et al. (1986). However their methods did not constrain the apparent resistivity and therefore have been uncommon.

The new phase tensor method uses the invariant approach from the apparent resistivity tensor method previously applied to bipole-dipole resistivity surveys (Bibby, 1986). The phase tensor can be represented as ellipses which show the relationship 
between the horizontal electric and magnetic fields. The basic characteristics of the phase tensor $(\underline{\underline{\Phi}})$ are:

- In 1-D there is a single co-ordinate invariant phase.

- In $2-\mathrm{D} \underline{\underline{\Phi}}$ is symmetric, and one principal axis is aligned with the strike.

- In 3-D $\underline{\underline{\Phi}}$ is non-symmetric and contains a third coordinate invariant "that provides a distortion free measure of the asymmetry of the regional MT response" (Caldwell et al., 2004).

Galvanic distortion of the regional EM field is virtually independent of the period, provided the period is large enough to overcome the effects of scale length, geometry and conductivity (Caldwell et al., 2004). Equation 3.51 shows the relationship between the regional and distorted fields. Splitting the impedance tensor into real and imaginary components (i.e. $Z=X+i Y$ ) it can be shown that

$$
\underline{\underline{X^{\prime}}}=\underline{\underline{C X}} R
$$

and

$$
\underline{\underline{Y}}^{\prime}=\underline{\underline{C Y}}_{R}
$$

where $\underline{\underline{X}}_{R}$ and $\underline{\underline{Y}}_{R}$ are the real and imaginary parts of the regional impedance tensor respectively and $\underline{\underline{X}}^{\prime}$ and $\underline{\underline{Y}}^{\prime}$ of the measured impedance tensor. In terms of a tensor, equation 3.46 for the phase can therefore be generalised as

$$
\underline{\underline{\Phi}}^{\prime}=\underline{\underline{X^{\prime}}} \underline{\underline{Y^{\prime}}}
$$

In this form the distortion matrices cancel out, leaving the phase tensor undistorted as shown below.

$$
\begin{aligned}
\underline{\underline{\Phi}} & =\underline{\underline{X}}^{-1} \underline{\underline{Y}} \\
& =(\underline{\underline{C X}})^{-1}(\underline{\underline{C Y}}) \\
& =\underline{\underline{X}}_{R}^{-1} \underline{\underline{C}}^{-1} \underline{\underline{C Y}} R \\
& =\underline{\underline{X}}_{R}^{-1} \underline{\underline{Y}}_{R} \\
& =\underline{\underline{\Phi_{R}}}
\end{aligned}
$$


This phase tensor may be represented as an ellipse described through a set of coordinate invariants. The invariants are described by a direction and three independent scalars that do not depend on the co-ordinate system. These scalars are represented as $\Phi_{\max }, \Phi_{\min }$ and the 'skew angle' $\beta$ (adapted from Bibby (1986)); where

$$
\begin{aligned}
& \Phi_{\text {max }}=\frac{1}{2}\left[\left(Z_{11}-Z_{22}\right)^{2}+\left(Z_{12}+Z_{21}\right)^{2}\right]^{\frac{1}{2}}+\frac{1}{2}\left[\left(Z_{11}+Z_{22}\right)^{2}+\left(Z_{12}-Z_{21}\right)^{2}\right]^{\frac{1}{2}} \\
& \Phi_{\text {min }}=\frac{1}{2}\left[\left(Z_{11}+Z_{22}\right)^{2}+\left(Z_{12}-Z_{21}\right)^{2}\right]^{\frac{1}{2}}-\frac{1}{2}\left[\left(Z_{11}-Z_{22}\right)^{2}+\left(Z_{12}+Z_{21}\right)^{2}\right]^{\frac{1}{2}}
\end{aligned}
$$

and

$$
\beta=\frac{1}{2} \tan ^{-1}\left(\frac{\Phi_{12}-\Phi_{21}}{\Phi_{11}+\Phi_{22}}\right)
$$

where $\Phi_{11}, \Phi_{12}, \Phi_{21}$, and $\Phi_{22}$ are the elements of the phase tensor $\underline{\underline{\Phi}}$. $\beta$ represents the "misalignment" between the electric and magnetic fields and is indicative of the degree of 3 dimensionality in the data. An angle, $\alpha$, which represents the angle between the co-ordinate axes and the principal axes of the symmetric phase tensor (Bibby, 1986) and is not invariant, is given by

$$
\alpha=\frac{1}{2} \tan ^{-1}\left(\frac{\Phi_{12}+\Phi_{21}}{\Phi_{11}-\Phi_{22}}\right)
$$

Additionally, $\Phi_{\max }$ and $\Phi_{\min }$ can be conveniently used to define the "ellipticity"

$$
\lambda=\frac{\phi_{\max }-\Phi_{\min }}{\phi_{\max }+\Phi_{\min }}
$$

In terms of the co-ordinate invariants the phase tensor can then be described as

$$
\underline{\underline{\Phi}}=\underline{R}^{T}(\alpha-\beta)\left[\begin{array}{cc}
\Phi_{\max } & 0 \\
0 & \Phi_{\min }
\end{array}\right] \underline{R}(\alpha+\beta)
$$

These properties of the phase tensor may be used to describe an ellipse for each period measured. "The principal axes of the phase tensor indicate the direction of the regional conductivity gradient at the length scale that depends on the period and conductivity structure concerned" (Caldwell et al., 2004). The length of the major 
principal axes is given by $\Phi_{\max }$, the minor principal axis is given by $\Phi_{\min }$, and the angle $\alpha$ is the angle between the x-axis and the preferred direction of the electric field as shown in the diagram below.

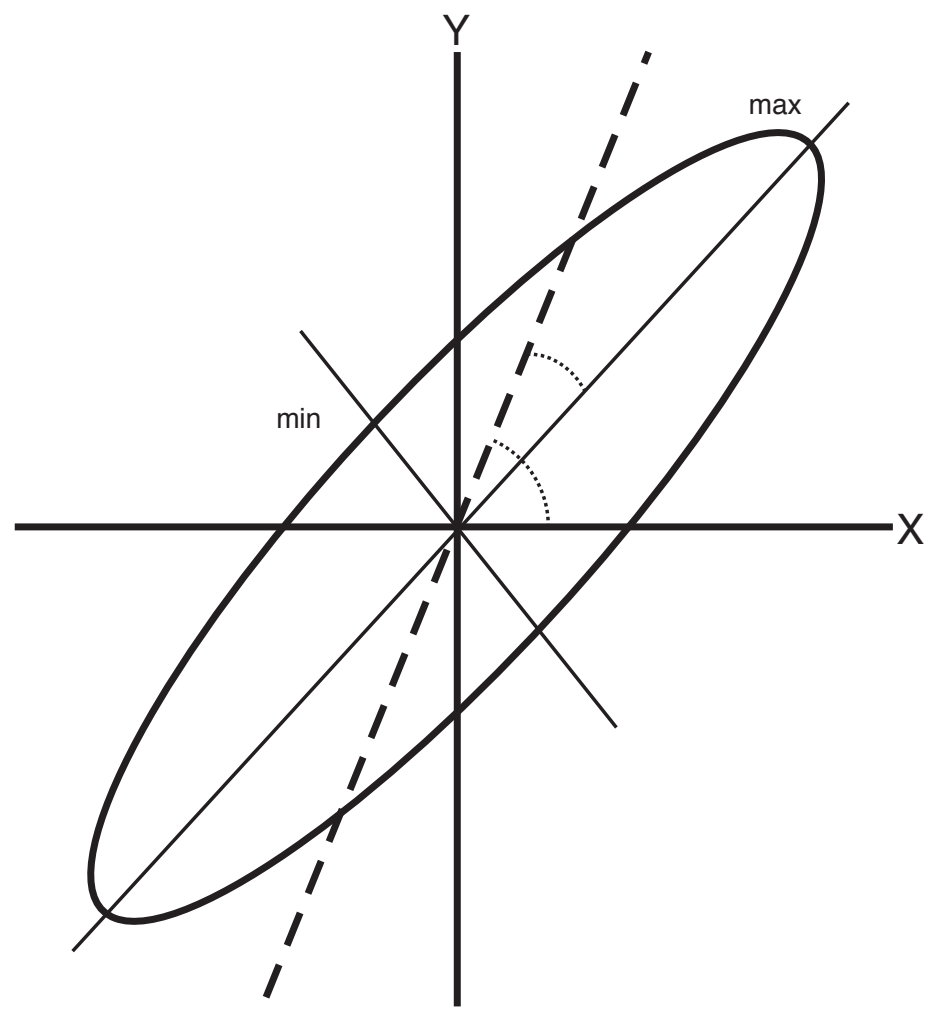

Figure 3.3 The parameters of the phase tensor ellipse as defined by Caldwell et al. (2004).

An ellipse representing the appropriate parameters may be constructed by plotting $\left(\gamma v_{x}+\right.$ easting $)$ versus $\left(\gamma v_{y}+\right.$ northing $)$, where $\gamma$ is an appropriate scale factor applied to every ellipse.

$$
\left[\begin{array}{l}
v_{x} \\
v_{y}
\end{array}\right]=\left[\begin{array}{ll}
\Phi_{11} & \Phi_{12} \\
\Phi_{21} & \Phi_{22}
\end{array}\right]\left[\begin{array}{c}
\cos \theta \\
\sin \theta
\end{array}\right]
$$

When a number of sites have been occupied, a map of the ellipses can be constructed that allows a spatial representation of this data at any particular frequency. Lateral variations of the structure can be easily identified from this graphical representation.

In 1-D conductivity structures the principal axes of the phase tensor ellipse are of the same length and the 'ellipse' will be a circle, additional properties are:

- The radius indicates the manner in which the resistivity varies with depth. That is, a large radius (radius $>1$ ) indicates a large phase which is indicative of a decreasing resistivity with depth and vice versa. 
- $\underline{\underline{\Phi}}$ is symmetric,

- $\alpha$ is undefined and unstable (Caldwell et al., 2004).

Where the structure is 1-D (both ellipticity and $\beta$ are approximately zero), the apparent resistivity can be recovered using the technique of Bibby et al. (In Press). The regional impedance is

$$
\begin{aligned}
\underline{\underline{Z}}_{R} & =\left[\begin{array}{cc}
0 & Z_{1 D} \\
-Z_{1 D} & 0
\end{array}\right] \\
& =Z_{1 D}\left[\begin{array}{cc}
0 & 1 \\
-1 & 0
\end{array}\right] \\
& =\left(X_{1 D}+i Y_{1 D}\right)\left[\begin{array}{cc}
0 & 1 \\
-1 & 0
\end{array}\right]
\end{aligned}
$$

The real part of the measured impedance tensor is given by

$$
\underline{\underline{X}}=\underline{\underline{C X}} R=\underline{\underline{C}} X_{1 D}\left[\begin{array}{cc}
0 & 1 \\
-1 & 0
\end{array}\right]
$$

It follows that

$$
\begin{aligned}
\underline{\underline{X}}\left[\begin{array}{cc}
0 & -1 \\
1 & 0
\end{array}\right] & =\underline{\underline{C}} X_{1 D}\left[\begin{array}{cc}
0 & 1 \\
-1 & 0
\end{array}\right]\left[\begin{array}{cc}
0 & -1 \\
1 & 0
\end{array}\right] \\
& =\underline{\underline{C}} X_{1 D}
\end{aligned}
$$

Thus the distortion tensor can be determined if $X_{1 D}$ can be estimated. If an estimate of $X_{1 D}$ is made as (for example)

$$
X_{1 D}=\sqrt{X_{11} X_{22}-X_{12} X_{21}}
$$

then an average value of $\underline{\underline{C}}$ may be calculated using a number of frequencies over which the data are 1-dimensional. The calculated distortion tensor may then be used to "strip" the distortion from the entire frequency range as

$$
\underline{\underline{Z}}_{R}=\underline{\underline{C}}^{-1} \underline{\underline{Z}}^{\prime}
$$

Note that $\underline{\underline{Z}}_{R}$ does not have to be 1-D over the whole frequency range, only that part 
of it used to calculate $\underline{\underline{C}}$.

This results in a similar situation to static shift as there is still an arbitrary constant multiplying the distortion tensor. A 1-D section of the curve (generally of high frequency data) is used to calculate the distortion tensor, which is then removed from the entire curve.

For a 2-D structure, equation 3.63 is redefined in terms of $\alpha^{\prime}$ instead of $\alpha$ where $\alpha^{\prime}=\alpha-\theta$ and $\theta$ is the rotation of the measurement axes from the strike rotation as in section 3.1.2. In this co-ordinate system the invariants become the TE and TM component phases, which can be represented by

$$
\underline{\underline{\Phi}}^{\prime}=\left[\begin{array}{cc}
\frac{Y_{\perp}}{X_{\perp}} & 0 \\
0 & \frac{Y_{\|}}{X_{\|}}
\end{array}\right] \text {or }\left[\begin{array}{cc}
\frac{Y_{\|}}{X_{\|}} & 0 \\
0 & \frac{Y_{\perp}}{X_{\perp}}
\end{array}\right]
$$

As before, prior information is required to determine which invariant relates to which mode. For a 2 -D structure $\beta \approx 0$, but $\beta \approx 0$ does not require the structure to be 2 -D. The angle of the major axis is given by $\alpha^{\prime}$, which by equations 3.61 and 3.65 means that $\alpha=\theta$ or $\alpha=\theta+90^{\circ}$, the strike or normal direction of the structural boundary.

In 3-D the major axis of the ellipse indicates the 'preferred' direction of the inducted current (Caldwell et al., 2004). Around a conductive cube in a resistive matrix for example, major axes would be aligned radially out from the cube, whereas near a resistive cube in a conductive matrix major axes would be aligned tangentially. In these cases $\beta$ will vary around the edges of the cube, being approximately zero on the symmetry axes and large near corners, i.e. near the major points of 3-dimensionality $\beta \neq 0$.

In summary, the dimensionality of the data may be determined from the behaviour of the parameters, $\lambda, \beta$ and $\alpha$.

- 1-D: $\beta \approx 0$ and $\lambda \approx 0, \alpha$ unstable.

- 2-D: $\beta \approx 0$ and $\lambda \neq 0$, the strike is then defined by a constant value of $\theta$.

- 3-D: $\beta \neq 0$ however the value of $\alpha-\beta$ will give the preferred current direction. 
The threshold values assigned to $\beta$ and $\lambda$ for approximation to zero are $\beta=3$ and $\lambda=0.15$. Although threshold values are determined at the discretion of the operator, these are the values applied to this study.

In order to remove distortion from a secondary curve, these parameters are plotted to find regions of the data that behave in a 1-D manner. The distortion matrix is calculated as outlined above for each frequency that is deemed to be 1-D. These matrices are then averaged to obtain a single distortion matrix that is representative of the distortion. The inverse of this matrix is then multiplied by the impedance tensor at each frequency measured in order to remove the galvanic distortion. This step has to be applied individually to each site occupied in a survey. A similar method can be used for a 2-D section of the curve to determine the distortion matrix for situations where there is no 1-D data (Bibby, 2004, pers.comm.).

Thus the phase tensor method allows distortions to be removed without the 2-D assumption that was required in earlier papers. It is therefore ideal to apply to a situation such as Mt. Ruapehu where the resistivity structure can be assumed to be 3-D.

\subsection{Processing}

Once the data have been collected they have to be processed before they can be modelled. There are currently a number of programs available that will process the data with minimal user input. This section outlines the steps taken in many of these programs.

Although each system differs, in this case (using a Phoenix MTU-2000 unit) a central computer collects data from three magnetometers, and four electrodes over the period of two days. Often the data collected are divided into frequency bands, one suggested division is shown in the table below.

Notch filters are set during recording so that frequencies can be excluded. One is commonly set for the $50 \mathrm{~Hz}$ power mains which affects most parts of New Zealand. The first step in processing is to adjust the magnetic data to fit the coil calibration 


\begin{tabular}{|c|c|c|}
\hline Band & Frequency range & Data collection rate \\
\hline \hline Band 1 & $1000-10 \mathrm{~Hz}$ & $3000 \mathrm{~Hz}$ \\
\hline Band 2 & $100-1 \mathrm{~Hz}$ & $300 \mathrm{~Hz}$ \\
\hline Band 3 & $10-0.1 \mathrm{~Hz}$ & $30 \mathrm{~Hz}$ \\
\hline Band 4 & $0.333-0.001 \mathrm{~Hz}$ & $1 \mathrm{~Hz}$ \\
\hline
\end{tabular}

Table 3.1

The common frequency

bands and collection

rates used in the

collection

magnetotelluric data

and to correlate the data with that from the remote reference. The comparison to the remote reference eliminates sections of the data shown to be noisy that are present at one site but not the other. These sections can be classed as noise because the natural EM field used is a plane wave and therefore homogeneous over the distance separating the site from the remote reference site.

Any long period trends are removed using linear least squares regression, or a high pass filter on each component. This is followed by a cosine bell being applied to the first and last ten percent of the data points to reduce the power leakage into the side lobes of the Fourier transform. Following the Fourier transform, auto- and cross-spectral estimates of the components are calculated and averaged. These spectral estimates represent smaller time segments of data and are used to calculate coherency and errors within the impedance tensor.

After the cross-spectral analysis, robust processing takes place (completed here by the program SSMT, which is proprietary software of Phoenix Geophysics; Toronto, Canada). This process is repeated with twenty varying sections of data so that for each frequency there are twenty sets of Fourier co-efficients. The Fourier co-efficients are used to calculate the correlation co-efficients so that the impedance tensor can be calculated at each frequency. The results from each of these calculations are put into another program, where the cross-powers can be edited to eliminate outliers (GEO, 2004). The idea here is to manually remove noisy time sections within the data. At each site the first and last sections are often noisy due to local disturbances associated with installing and removing the site. Obviously there is a risk of introducing a bias and for this reason only the most extreme points are removed. As a result many noisy data points may still exist within the data set, particularly in the dead band, $(10-0.1$ $H z)$ in which the natural signal level is low. 
At this point, this study differs from the standard procedures. A Matlab (Mat, 1992) script prepared by Dr. Bibby (2004, pers.comm.) has been used to calculate the parameters defined in Caldwell et al. (2004) and discussed in section 3.3.5. The ellipticity is used along with the value of $\beta$ to determine which parts of each curve are 1-D. This is then used to calculate the distortion tensor and remove the distortion from the entire curve. The output of Bibby's Matlab script includes nine graphs that represents the data set. These are:

1. the original data curves as defined in standard procedures,

2. the ellipticity, $\beta$, and $\alpha-\beta$ (strike) as a function of period,

3. phase tensor ellipses for every second frequency,

4. overlapping distortion ellipses for the 1-D section,

5. data curves after distortion has been removed.

At this point the data are ready to be modelled and interpreted.

\subsection{Modelling}

Once the data have been processed, the apparent resistivity and phase curves are used to calculate an initial estimate of the conductivity structure of the area. For data that is 1-D a Bostick inversion (Bostick, 1977) is a common method used to invert the data to derive the 1-D structure. The inversion scheme actually solves for the conductance, which is the product of the conductivity and thickness. There is no unique solution, although there are limits as to what is reasonable.

Using the simple inversion scheme proposed by Niblett and Sayn-Wittgenstein (1960), the depth is calculated from

$$
h=\left(\frac{\rho_{a} T}{2 \pi \mu_{0}}\right)^{2}
$$


where $\rho_{a}$ is the apparent resistivity at period $T$. The conductivity at that depth is then calculated to be

$$
\sigma(h)=\frac{1}{\rho_{a}}-\frac{h}{\rho_{a}^{2}} \frac{\partial \rho_{a}}{\partial h}
$$

Thus if $\rho_{a}$ is known as a function of the period $(T)$ the complete conductivity structure can be calculated. This method later evolved into the Bostick inversion (Bostick, 1977).

There are two major types of full 1-D inversions; those which try to fit a continuous curve to the structure and those that use as few layers as possible to match the data. Both types of model use the mathematics described earlier to calculate the structure. Models also vary in whether they attempt a good mathematical fit or a smooth realistic model. Structures that are not strictly 1-D can be modelled by modelling the determinant of the impedance tensor, which is invariant with direction and thus a comparable value.

Two 1-D modelling schemes were used in this study. The Occam inversion scheme (Constable et al., 1987) uses a large number of thin layers in which case unnecessary low resistivity layers are not required to fit the structure as in many schemes. A more mathematical fit is achieved by IPI_MT (Bobachev, 1990). This method shows the observed and theoretical data during the calculation, and the structure can be manually adjusted from the best mathematical fit to a more realistic structure once the program has run.

In the more complicated situations where the structure is 2 or $3-\mathrm{D}$ numerical routines are used. Many of these have been developed and are in common use, for example Rodi and Mackie (2001), Newman and Alumbaugh (2000), Mackie and Madden (1993), or Smith and Booker (1991). These programs are not only required to solve the induction equations but must simultaneously solve for all the model boundary conditions as well.

There are three main types of numerical methods:

- finite difference methods,

- finite element methods, 
- integral equations.

The first two are forward modelling techniques that calculate the theoretical response to the structure, while the third involves integrating the differential equations to calculate the response. For 2-D structure, inversion techniques are more common. The basic process is to start by assuming a conductivity structure, often a uniform half space. The theoretical data are forward modelled, compared to observed data, and the structure altered until the comparison shows that the calculated data are within the error stated by the operator. This method usually can resolve a number of robust and smoothed inversions. The robust inversions find the best mathematical fit, which is often not geologically reasonable. The smoothing inversions will smooth extreme structure produced by the robust inversions and create a more geologically plausible solution although the fit to data is somewhat decreased.

3-D algorithms work in much the same way as the 2-D algorithms, although until recently the power and speed of computers has limited the use of these algorithms. However, the prohibitive element in many cases is usually the lack of data collected with respect to the area of the survey (Newman and Alumbaugh, 2000).

In order to make the horizontal conductivity smooth, horizontal nodes are uniformly spaced where there are sites, but are spaced logarithmically outside the data region. Adding nodes between the sites only makes a significant difference if the sites are very far apart or quite uneven (Smith and Booker, 1991).

Although all models solve the equations presented earlier in this chapter, the nonuniqueness of the modelling techniques can result in different final models depending on the techniques selected in each particular study. 
CHAPTER 4

\section{THE DATA SET}

The data gathered for this study were collected in a joint operation between the Institute of Geological and Nuclear Sciences (GNS), Tokyo Institute of Technology (TIT), and Victoria University of Wellington (VUW). The project is intended as a preliminary study for a proposed extensive 3-D study later this decade. Data were collected from 24 sites, broadly in a scattered line southeast - northwest over the northern flank of Mt. Ruapehu in late February 2004. In order to compare the volcanic environment with the surrounding geologic setting, the extent of the line includes both the eastern and western boundaries of the Tongariro Volcanic Centre (TVC).

During the time period of data collection there was no reported evidence of volcanic or seismic activity within the TVC (Geonet, 2004). The website for the National Environmental Satellite, Data, and Information Services (2004) reported that this was also a period when the Dst indices (see definition below) indicated that the magnetic activity was very low (see figure 4.1).

Dst indices: The Disturbance storm time equivalent equatorial magnetic disturbance indices (Dst) are derived from hourly scalings of low-latitude horizontal magnetic variation. They show the effect of the globally symmetrical westward flowing high altitude equatorial ring current, which causes the "main phase" depression worldwide in the H-component field during large magnetic storms. Hourly H-component magnetic variations are analyzed to remove annual secular change trends from records of a world wide array of low-latitude observatories. A cosine factor of the site latitude transforms residual variations to their equatorial equivalents and harmonic 


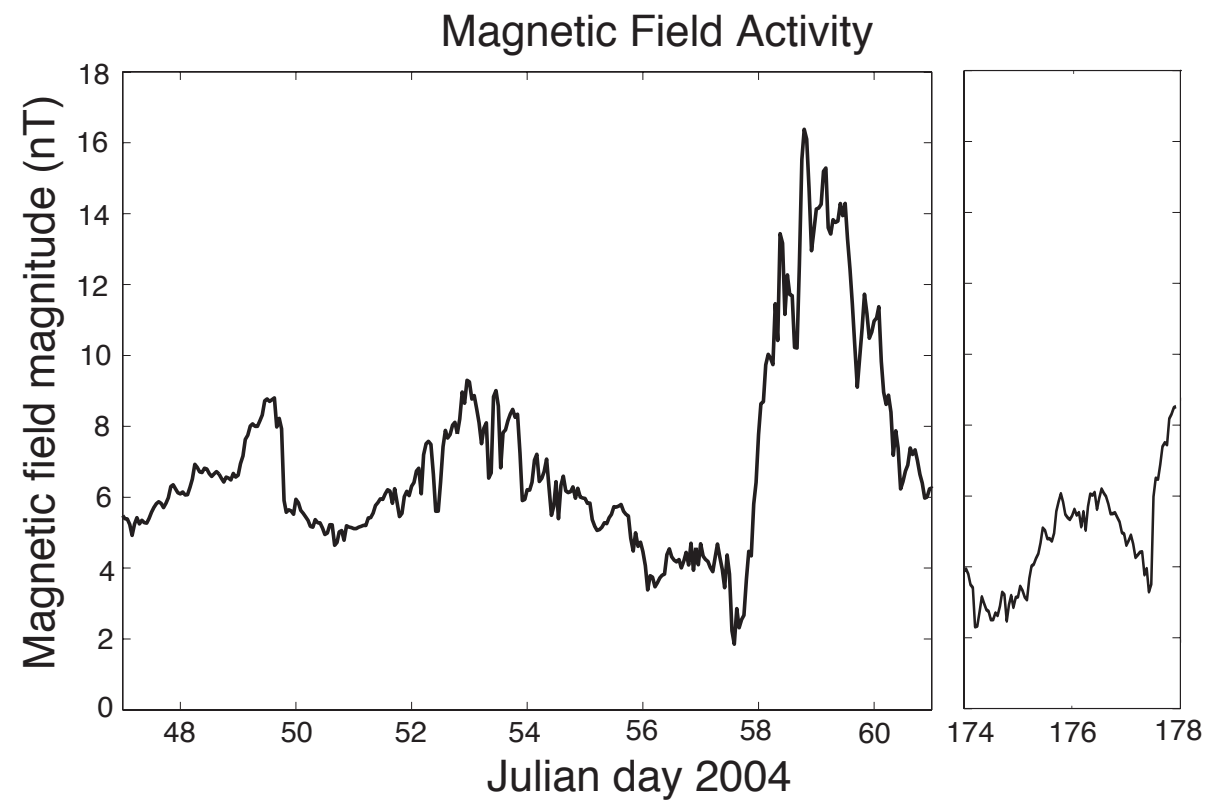

Figure 4.1 The level of terrestrial magnetic activity recorded during fieldwork.

analysis isolates the term used as the Dst index. (National Enviromental Satellite Data and Information Services, 2004)

Activity remained below the 2003 yearly average (of $26.5 n T$ ) for the survey, dropping to less than $10 \%$ of that average on the $21^{\text {st }}$ of February. The low level of activity accounts for the observed low signal to noise ratio within the dead band (frequencies ranging from $0.1-10 \mathrm{~Hz}$ ).

In addition to the low electromagnetic activity levels during this period the lower and central parts of the North Island were battered by unusually fierce storms. Some sites were set up and occupied during thunderstorms, gale force winds, snow and intense rain, all of which are potential noise sources and increase the risk of mistakes during set up. Many areas in this part of the country were in a state of emergency due to flooding and slips. Fortunately only two sites in the east were inaccessible. Both these sites were occupied in late June by GNS to complete the study.

Despite the poor weather conditions and low source signal, the data collected were considered to be of reasonable quality once extreme outliers were eliminated during processing. 


\subsection{Data Collection and Processing}

The 24 sites were occupied with four Phoenix MTU-2000 units, two from TIT and two from GNS. A further GNS unit was set up in the Kaingaroa Forest, approximately 130 $\mathrm{km}$ to the north as a remote reference site. The sites approximately form two profile lines, one running in a southeast - northwest direction over the northern flank and the other perpendicular on the southeast flank of the volcano. The exact locations of the sites are shown in figure 4.2 with additional details presented in table 4.1.

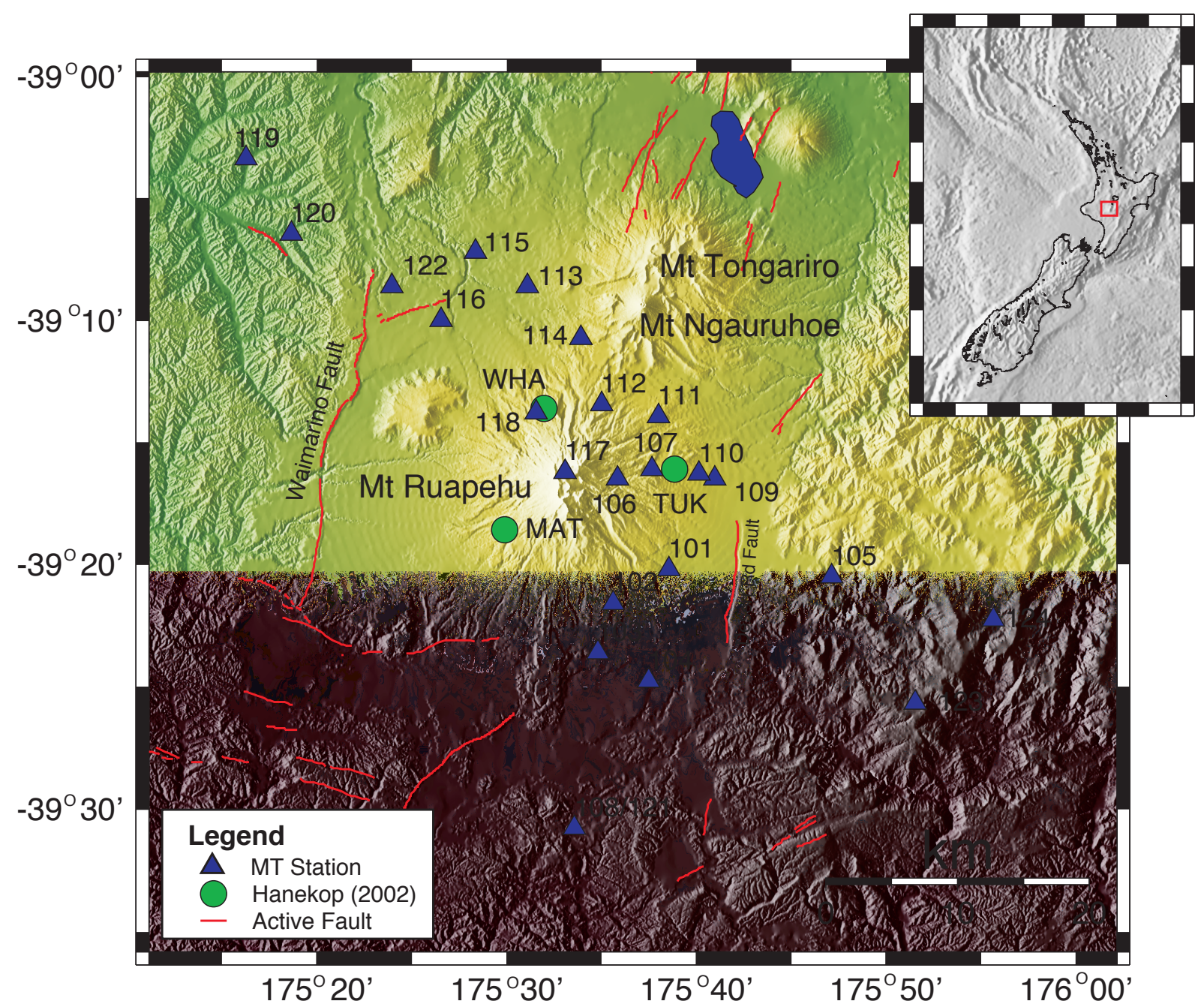

Figure 4.2 The location of magnetotelluric sites occupied during the 2004 Mt Ruapehu MT survey. Original map created by Horspool (2004, pers.comm.)

Although it is desirable to occupy each site for two nights with a continuous remote reference, this was not always possible. Due to practicality or access limitations some 


\begin{tabular}{|c|c|c|c|c|}
\hline \multicolumn{5}{|c|}{ The sites measured during the $2004 \mathrm{Mt}$. Ruapehu MT survey } \\
\hline Site & Easting & Northing & Altitude & Instillation date/Comments \\
\hline Remote & 2806201 & 6280130 & 600 & $16 / 02 / 04$ \\
\hline MTR-101a & 2738698 & 6203821 & 1000 & $17 / 02 / 04$ \\
\hline MTR-102a ${ }^{\dagger}$ & 2734688 & 6201372 & 1033 & $19 / 02 / 04$ \\
\hline MTR-103a & 2733423 & 6197698 & 866 & $18 / 02 / 04$ \\
\hline MTR-104a & 2737191 & 6195420 & 836 & $18 / 02 / 04$ \\
\hline MTR-105a ${ }^{\dagger}$ & 2751097 & 6203194 & 1013 & $19 / 02 / 04$ \\
\hline MTR-106a & 2735264 & 6210725 & 1705 & $20 / 02 / 04$ \\
\hline MTR-107a & 2737928 & 6211400 & 1508 & $20 / 02 / 04$ \\
\hline MTR-108a & 2731276 & 6184478 & 707 & Site reoccupied as MTR-121a \\
\hline MTR-109a & 2742658 & 6210554 & 1154 & Site relocated to MTR-110a \\
\hline MTR-110a & 2741490 & 6210994 & 1210 & 22/02/04 One night only \\
\hline MTR-111a & 2734260 & 6216442 & 1455 & $23 / 03 / 04$ \\
\hline MTR-112a & 2738539 & 6215426 & 1362 & $23 / 02 / 04$ \\
\hline MTR-113a ${ }^{\dagger}$ & 2728911 & 6223957 & 955 & $24 / 02 / 04$ \\
\hline MTR-114a ${ }^{\star \dagger}$ & 2732832 & 6221392 & 1200 & $24 / 02 / 04$ \\
\hline MTR-115a & 2725024 & 6228284 & 834 & $25 / 02 / 04$ \\
\hline MTR-116a & 2722278 & 6223181 & 892 & $25 / 02 / 04$ \\
\hline MTR-117a & 2731363 & 6211426 & 2584 & 26/02/04 One night only \\
\hline MTR-118a ${ }^{\star \dagger}$ & 2729293 & 6216001 & 1459 & $26 / 02 / 04$ \\
\hline MTR-119a & 2707803 & 6235874 & 310 & $27 / 02 / 04$ \\
\hline MTR-120a & 2711089 & 6230040 & 321 & $27 / 02 / 04$ \\
\hline MTR-121a & 2731276 & 6184478 & 707 & $28 / 02 / 04$ One night only \\
\hline MTR-122a * & 2718626 & 6225886 & 780 & 28/02/04 One night only \\
\hline MTR-123a & 2757307 & 6193160 & 1200 & $22 / 06 / 04$ \\
\hline MTR-124a & 2763430 & 6199233 & 1083 & $22 / 06 / 04$ \\
\hline
\end{tabular}

Table 4.1

Detailed locations of each of the sites occupied during the 2004 Mt. Ruapehu MT survey. Sites annotated with a ${ }^{\dagger}$ indicate sites that were being occupied during a disk change at the remote reference, meanwhile sites annotated with a * indicate sites that were processed using the magnetics of an accompanying site (see text for details).

sites were removed after only one night. Once the data had been collected, the time series were viewed to locate any sections of bad data. The processing was carried out as described in section 3.4 using the computer programs SSMT (proprietary software of Phoeniox Geophysics; Toronto, Canada) and WinGLink (GEO, 2004).

As the remote reference site did not have the battery or memory capacity to run continuously for the entire survey, a number of sites were recording during the disk change at the remote reference site. As SSMT only allows one remote reference file the 
program had to be run twice, once for each file. The output of the SSMT program is an MT file that contains twenty cross-power spectral values for each frequency. The two files could be combined together in WinGLink, edited and saved as a single file (SEG, 1991) for the remaining processing steps.

It was also noted at the removal of one site that a number of porous electrode pots had cracked ceramic plates. The repercussions of the cracked plates are not known, and these electrodes were replaced as a matter of caution. When the raw data were viewed there were no indications that the data had been negatively effected.

An internal electrical connection within one $H_{x}$ coil also became disconnected in the middle of the survey. The plug attaching the magnetometer coil to the cable carrying the signal to the MTU-2000 computer broke, so no signal was being received from the coil. Unfortunately the protective plastic wrap that surrounded the coil prevented the disconnection from being detected until the final equipment clean up. By this time the $H_{y}$ coil from the same set had also become disconnected in a similar manner. From the raw time series data it was obvious that the coils became disconnected between site occupations. The three sites effected were MTR-114a, MTR-118a, and MTR-122a.

As the magnetic field is assumed to be a planar horizontal wave (section 3.1.1) the magnetic signals should be consistent between simultaneously occupied sites. Therefore, where a magnetic signal was not received, the site was processed using the magnetic data from the other site that was occupied simultaneously. Even though only one coil was broken during occupation of sites MTR-114a and MTR-118a, and two during MTR-122a, all three magnetic components were replaced for processing.

Site MTR-118a did not have a companion site recording for its entire time period and the remote reference data were split over two files. Thus site MTR-118 was processed twice, once using the magnetics from site MTR-117a and the earlier remote reference file, then a second time using the magnetics from site MTR-120a and the second remote reference file. When the two sets of cross-powers were combined after processing there was a distinct difference between the apparent resistivities calculated from each run of SSMT. This is probably due to inductive distortion created by topography for which there are currently no removal methods. Unfortunately this 
incompatibility reduces the credibility of substituting magnetic components between sites. The correct apparent resistivity is considered to be somewhere between the two sets of results. The data from the second run was labelled MTR-118b and has been used in the modelling phases of this study, as the magnetics are deemed to be less effected by topographic induction.

The two eastern sites, MTR-123a and MTR-124a were occupied in June. For practicality reasons there was no remote reference occupied at the same time. These sites were remote referenced against each other, although this did not reduce the effects of a significant noise contamination, presumably originating nearby but to date not identified.

In many situations cultural noise cannot be avoided as most infrastructural features release an electrical current into the Earth. These currents effect the electrical signal but not, in general the magnetic signal. When a current leaks into the ground it "creates" an electric field which is the noise. In general as $j=\sigma E$, for a given $j, E$ will be larger if $\sigma$ is smaller i.e. electric field noise is more of a problem in a resistive environment than in a conductive one. There are lots of examples of this - the most famous one being noise from the electric train system in Italy (Iliceto and Santarato, 1999). As the apparent resistivity is calculated from the ratio of the electrical and magnetic fields (refer to section 3.2) this leads to an error in the calculated value.

Although it is possible to set notch filters to remove certain constant frequencies of noise, not all sources are constrained to small frequency bands. Most cultural noise is the result of an unbalanced power network. This is often most obvious near small towns in the early evening (Ádám et al., 1986) when electricity consumption increases. The main causes of cultural noise are:

- mains power line (50 $\mathrm{Hz}$ in New Zealand),

- electric fences,

- large consumers being switched on or off,

- television receivers, 
- anti-corrosion systems on pipelines,

- vehicle movement,

- agricultural equipment,

- microwave stations,

- and telluric connection wires swaying in the wind.

From this list it can be seen that it is virtually impossible to avoid all noise sources when identifying a site location. However many of these noise signals are deemed to have been removed after remote referencing and a galvanic distortion removal technique have been applied.

\subsection{Apparent Resistivity and Phase Curves}

Prior to modelling the data it is useful to get an overview of the shape of the data and also to analyse the data parameters that define the dimensionality of the structures to be modelled. The data from any MT survey are presented as apparent resistivity and phase curves as a function of period. In this case these graphs were created by a Matlab script (Bibby, 2004, pers.comm.) that calculates and plots the data and parameters used to describe the sounding curves. This script also applies the phase tensor distortion removal process obtaining the undistorted data. The aim of this section is to present and discuss the main features of the undistorted apparent resistivity and phase curves and the implications of these features in determining the structure of Mt. Ruapehu.

An example of the data and parameters that are compiled by the Matlab script are presented here in five graphs as figure 4.3, similar graphs for each of the sites are presented in appendix A. The apparent resistivity and phase curves presented in figure 4.3 and appendix A are those after the distortion removal process has been applied. The other three graphs in figure 4.3 represent the ellipticity, $\beta$ value, and strike direction.

The shapes of the apparent resistivity and phase curves are sufficiently consistent between some sites the that data set could be split into a number of groups based on 

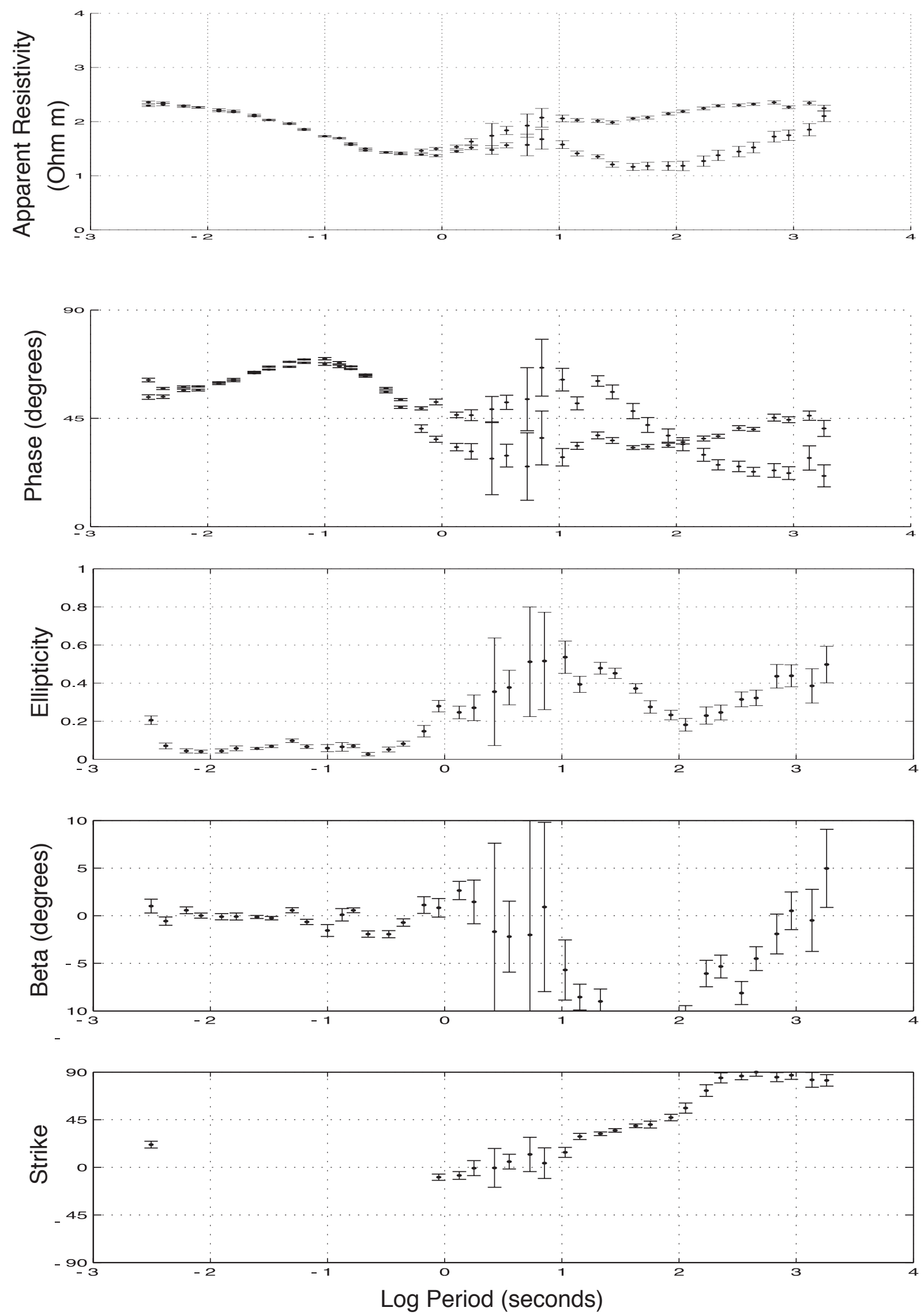

Figure 4.3 An example of the graphs created by plotting the data and phase tensor parameters, site MTR-106a 


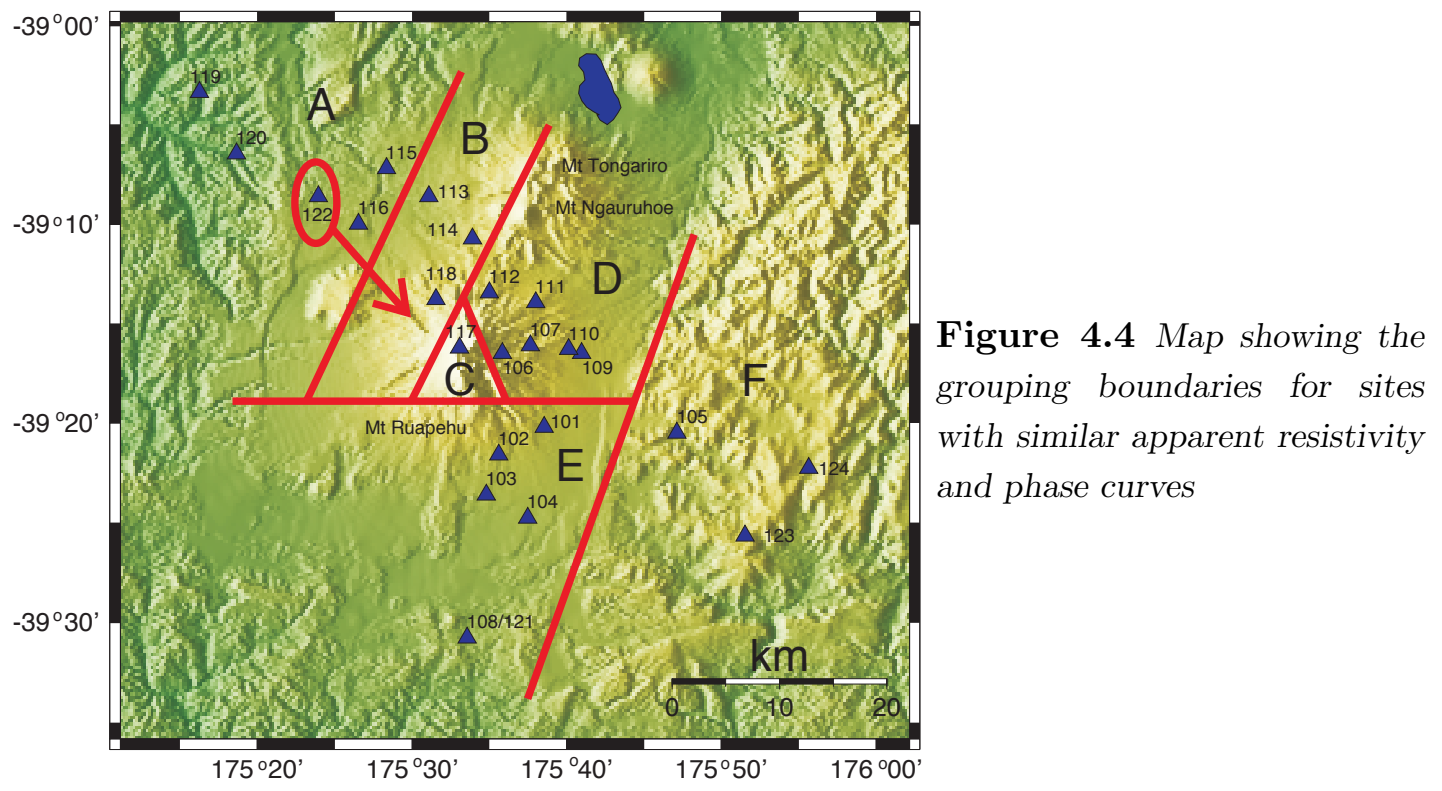

the shape of the curves. The area has been divided into six groups (figure 4.4), within which the curves exhibit similar properties. These groups are discussed individually.

The apparent resistivity and phase curves are each shown as two data sets, normally referred to as the TE and TM mode as they are below. The TE and TM modes refer to the $E$ and $B$-polarisations as in section 3.2, which in reality can only be determined after the data has been rotated to the strike direction. In this case the data are unrotated and the TE mode is used to refer to an electric field aligned north/south and the TM mode to an east/west aligned electric field, not necessarily the actual $E$ and B-polarisation directions.

\subsubsection{Group A}

Group A (figure 4.5) consists of the four western sites that do not show evidence of a shallow underlying relative conductor as seen at other sites. At these sites there is instead an indication of a relatively conducting surface. The apparent resistivity remains constant for both modes at periods less than $0.1 s$ at three of the four sites. Beyond this period the apparent resistivity increases for all sites. The TE mode curve increases linearly at an angle of $\approx 30^{\circ}$ out to the longest period recorded.

The TM mode is not as consistent between the sites. The sites west of the Waimarino Fault (see figure 4.2) have a steep increase in apparent resistivity out to $100 \mathrm{~s}$ at which 

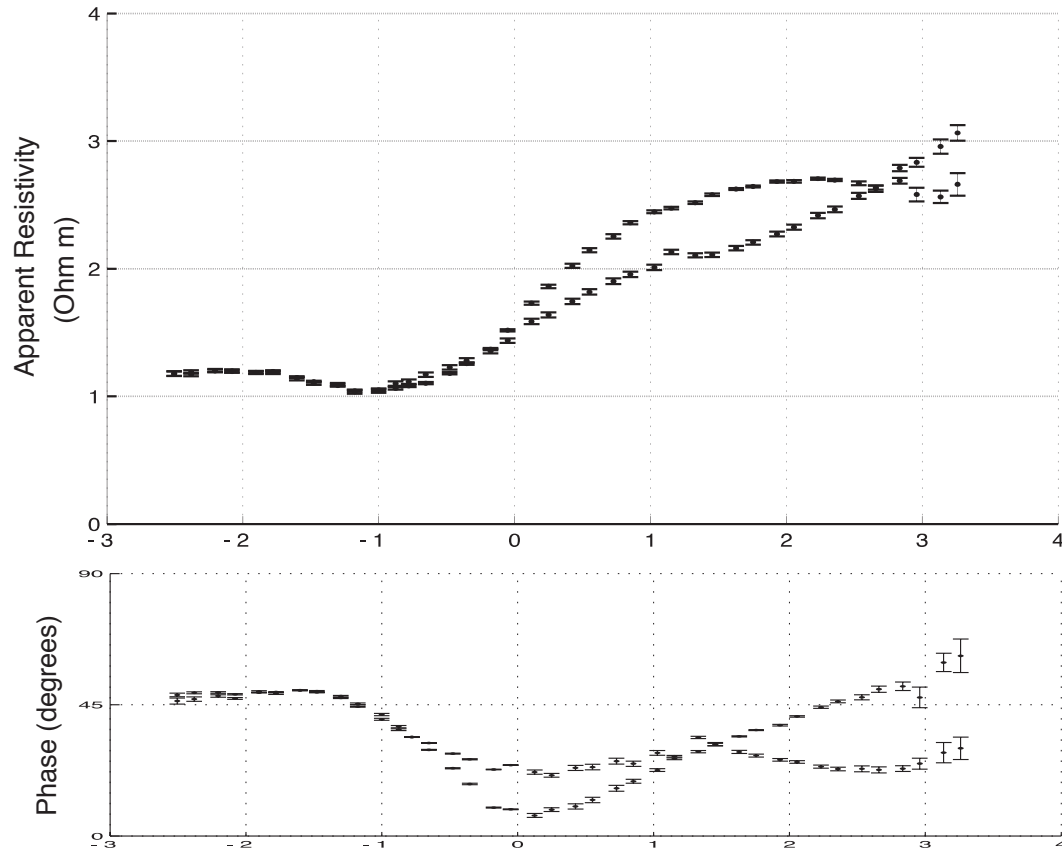

Log Period (seconds)
Figure 4.5 An example of the apparent resistivity and phase curves from Group A (site MTR-119a)

period the curve flattens out and crosses the TE mode curve as in figure 4.5. However on the eastern side of Waimarino Fault the TM mode increases linearly at a steeper angle to the TE mode and does not flatten out.

The phase curves show a similar pattern at each site; especially in the TE mode, although the pattern is less defined at site MTR-115a. The TM mode phase again has more variation between the eastern and western sides of the Waimarino Fault. As both modes have phases that are below $45^{\circ}$ at long periods it can be assumed that the resistivity is increasing with depth.

The implications of a constant TE mode and varying TM mode is that any structures in the area must be aligned north/south. This structure is most influential at periods around $1 \mathrm{~s}$, which corresponds to an approximate skin depth of $1.6 \mathrm{~km}$. As the TM mode is different on either side of the Waimarino Fault, this is the most likely candidate for the north/south structure influencing these sites.

\subsubsection{Group B}

Group B (figure 4.6) lies east of Group A on the western flank of the volcano. Site MTR-122a has been included in Group B although it geographically lies within Group A 
because the shape of the curves are more consistent with those in Group B. This group contains two sites that need to be interpreted carefully. Site MTR-118b contained almost no 1-D data, resulting in very few distortion matrices being averaged in the distortion removal process. This may have introduced a bias into the data. Site MTR122a contains large error bars and scatter. Some of this scatter may be due to the proximity of the New Zealand Main Trunk railway line or the shorter recording time. Each of these sites shows evidence of a surface resistor underlain by a conductor -
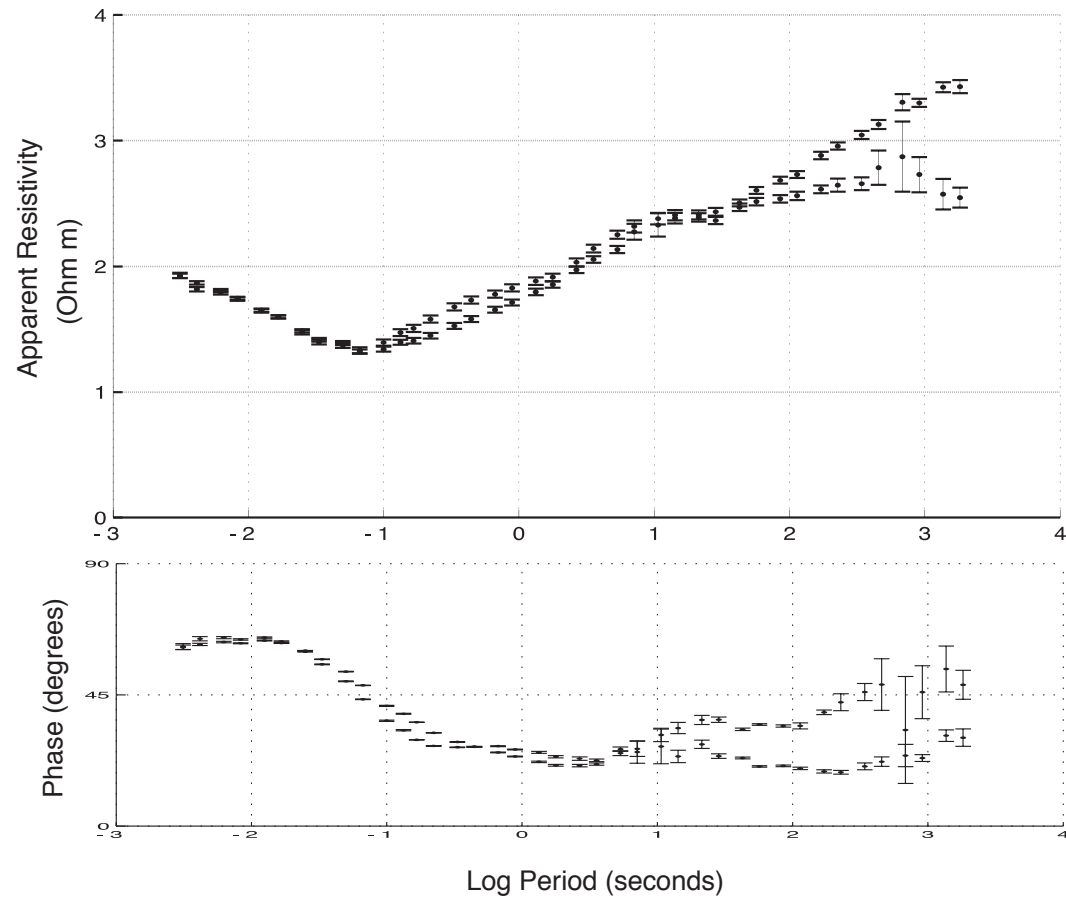

Figure 4.6 An example of the apparent resistivity and phase curves from Group B (site MTR-113a)

a minimum in apparent resistivity at a period of $0.1 \mathrm{~s}$. The difference between the resistor and conductor is consistently an order of magnitude with the minimum in the curve again corresponding to a skin depth of approximately $1.6 \mathrm{~km}$. At longer periods the apparent resistivity increases steadily with period. Again the TE mode increases linearly to the longest period while the TM mode flattens off at $1 s$ as seen in figure 4.6 .

The phase curves for each of the sites are the same to within uncertainties. The modes follow each other until they split at $10 s$ where the TM mode flattens out. As with the apparent resistivity curves, the phase curves indicate that below the shallow minimum, the resistivity continues to increase with depth.

This group of curves do not show any extreme changes that would be inconsistent 
with the surrounding groups. From the consistency of the graphs within this group it is reasonable to conclude that the same structural features influence each site. Additionally it is unlikely that there are any large structural boundaries within the area. The most important feature noted is the existence of the relative conductor below the more resistive surface layer.

\subsubsection{Group C}

The shape of the apparent resistivity curve for the site occupied on the summit plateau is considerably different from any other site. Therefore it has been placed in a group by itself; Group C (figure 4.7). As can be seen in figure 4.7 this site shows a surface resistor underlain by a relative conductor as in the last group. However in Group B the apparent resistivity started to increase after the period reached $0.1 s$, this is not the case for this site. Lower apparent resistivities are present throughout a much larger period range. As the period increases the resistivity does increase but at a much shallower gradient than other sites.

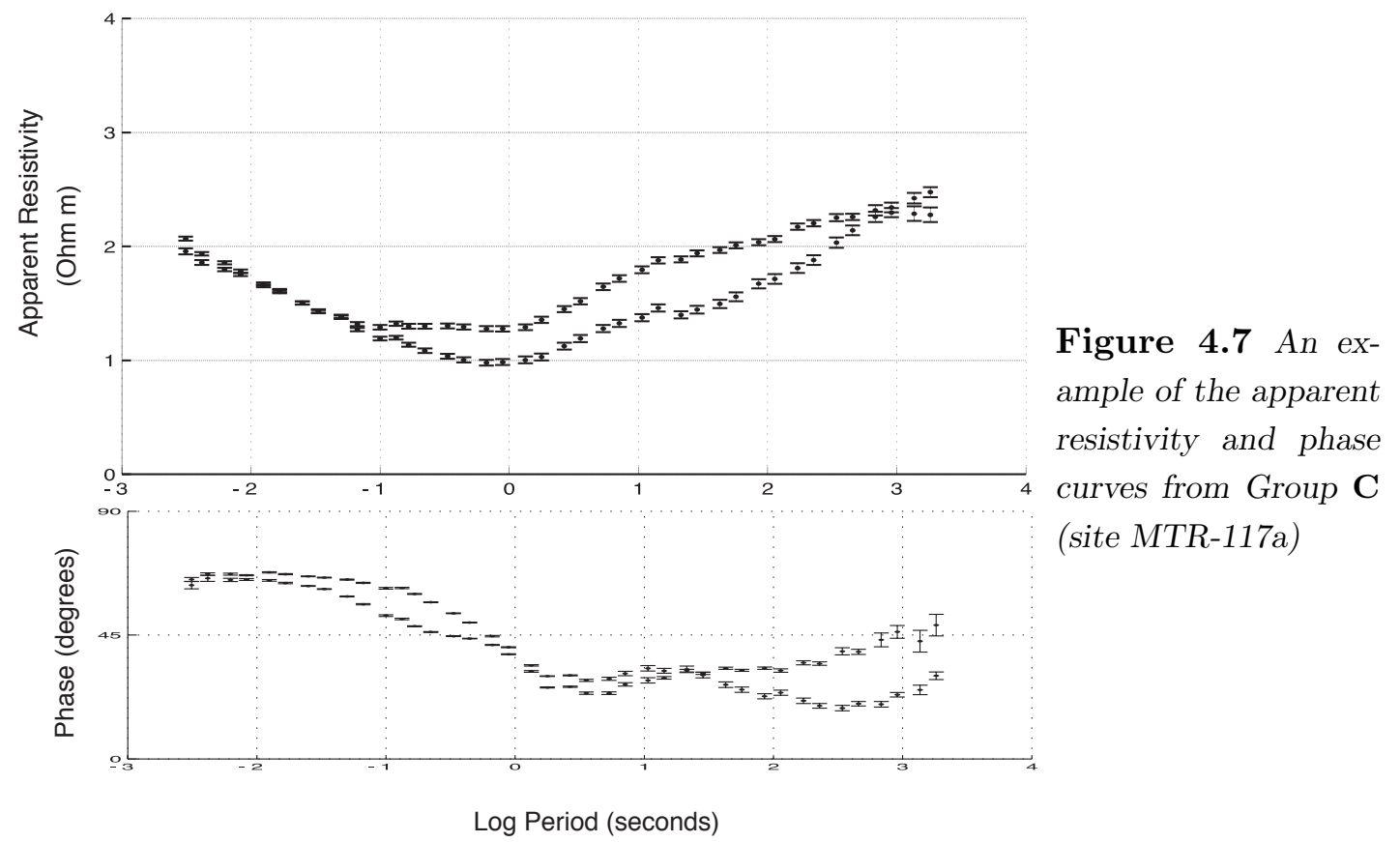

The phase curves have a very similar shape to phase curves from Group B. The biggest difference being that the TE and TM modes are less coherent than those in the other groups. This implies that the site contains a large degree of non 1-D structure. 
This site is also at a higher altitude and topographical effects are more prominent. Although the conductor may be related to the one observed in the other sites, it is perhaps more likely that it is due to the hot acidic crater lake and vent system nearby. Thus the interpretation of this site needs to be considered separately from other sites.

\subsubsection{Group D}

The next group (Group D, figure 4.8) covers the eastern flank of the volcano. As in Group B the curves from this group indicate a more resistive surface layer overlaying a relative conductor. The surface resistor present in these sites have a higher resistivity than those in Group B. It is also reasonable to assume that the thickness of the surface layer is greater as the minimum in apparent resistivity is not reached until $0.3 s$ and the conductivity contrast is greater (as seen in figure 4.8).

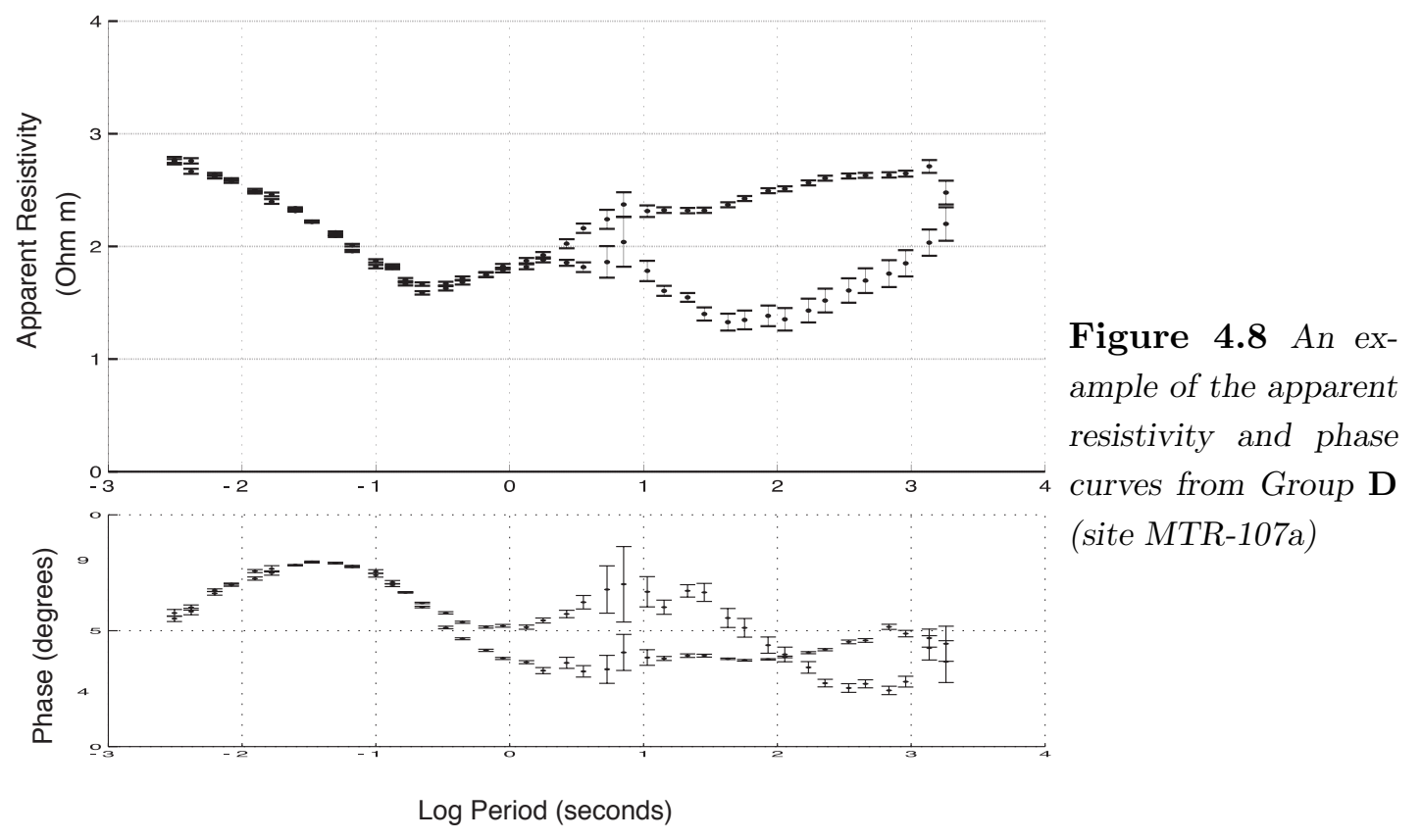

For these sites there is a split in the modes at $1 \mathrm{~s}$. The TM mode apparent resistivity follows a similar pattern to that of Group B, while the TE mode is vastly different. The TE mode starts to increase slightly then drops down to a strong minimum at 100 $s$ period before increasing again to join the TM mode curve.

The phase curves all share a similar pattern at high frequency before splitting at $0.1 \mathrm{~s}$. The TM mode is similar to Group B throughout the whole frequency range but 
the TE mode differs. Each site shows that the TE phase increases to a maximum at 10 $s$ (corresponding to the observed minimum in apparent resistivity). The magnitude of the maximum varies between sites, being largest at the high altitude sites. Following this maximum the TE phase at all sites dips to a minimum.

The basic interpretation is that there is a relatively resistive layer of varying thickness overlying a relative shallow conductor. Below this shallow conductor the TM mode is similar to those in Group B indicating that there is little north/south variation in the structure on either side of the volcano. However there is a significant change in the TE mode indicating that induced current in an east/west direction cross an electrical boundary. This boundary is associated with the presence of a relatively conductive north/south structure beneath the eastern flank.

\subsubsection{Group E}

South of the previous group is Group E (figure 4.9). These sites are on the lower southeastern flank of the volcano, and are very similar to those in Group D. The biggest difference being the surface resistivity and the magnitude of the resistivity contrast between the surface layer and the subsequent minimum in apparent resistivity of which are lower, as seen in figure 4.9. Although the longer period minimum in the TE mode is still present, the dip is not as great and decreases with latitude to be a level step in site MTR-121a.

Again the phase curves are similar to those in Group D. Site MTR-121a has a smaller phase variation than the other sites, and does not include the initial section of large phase values present in other curves. This site may possibly fit better with sites MTR-119a and MTR-120a to the far northwest.

The conclusion reached from looking at these sites is that the more resistive surface is either less resistive or thinner than the surface layer identified in Group D. As this surface layer is presumably volcanic eruptives it would be expected to be thinner further from the vent. Otherwise there is little difference between Groups D and E. 

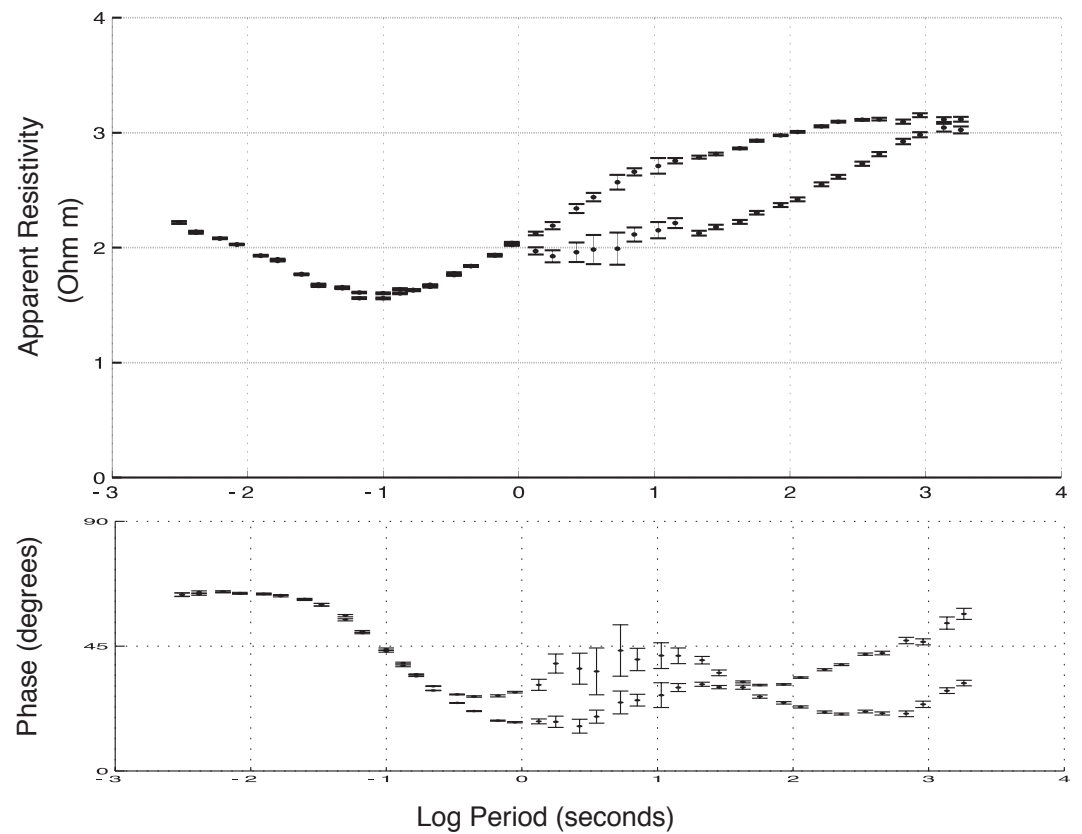

Figure 4.9 An example of the apparent resistivity and phase curves from Group $\mathbf{E}$ (site MTR-103a)

\subsubsection{Group F}

The final group (Group F, figure 4.10) comprises of the three sites to the east of the area outside the TVC boundary. These sites vary greatly from the other sites discussed previously. The biggest difference is that the apparent resistivity does not vary much over the entire frequency range. Despite the fact that there is a slight rise, dip then rise again, the resistivity hovers around $1000 \Omega m$ (as seen in figure 4.10).
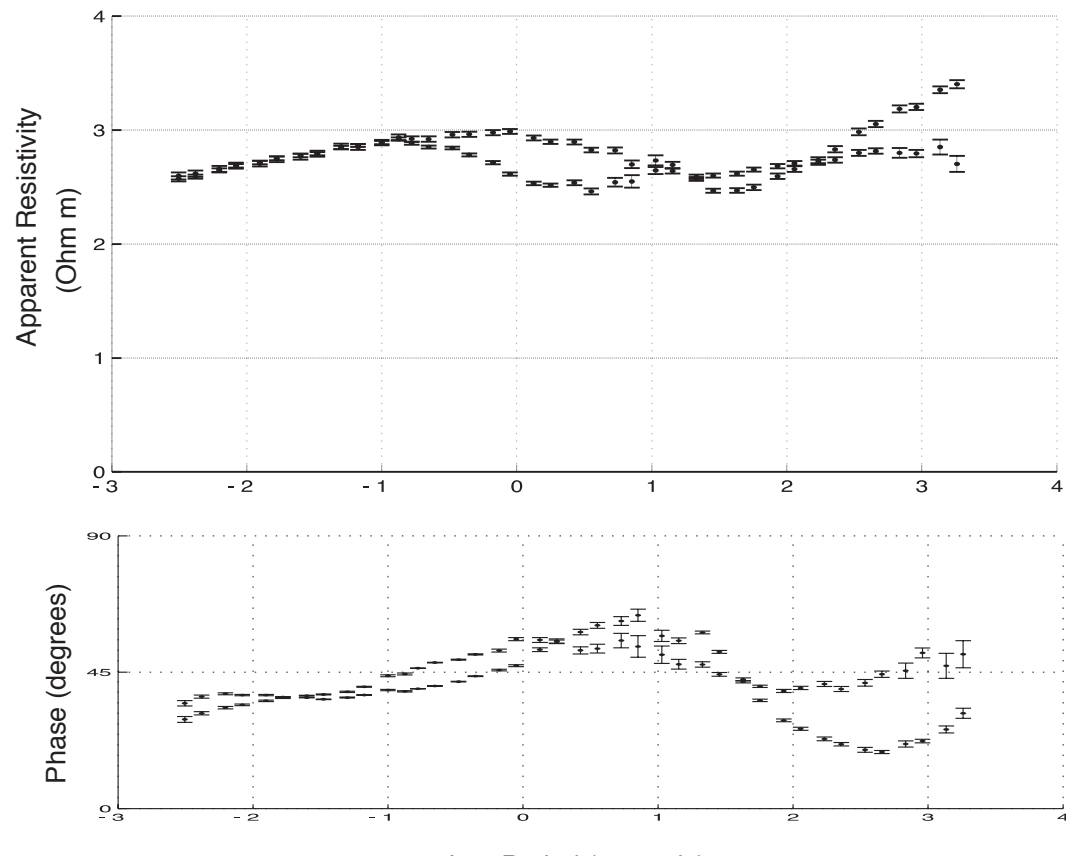

Figure 4.10 An example of the apparent resistivity and phase curves from Group $\mathbf{F}$ (site MTR-105a) 
There is also very little difference between the TE and TM modes. The apparent resistivity minimum present at $100 \mathrm{~s}$ in the sites on the eastern side of the volcano (Groups D and E) may be equivalent to the dip in resistivity at $30 s$ in this group. Due to the higher surface resistivity, the skin depth (equation 3.9) still places this conductor at a similar depth although the drop in resistivity is slight, dropping less than an order of magnitude.

The phases contain large errors for sites MTR-123a and MTR-124a, probably due to the lack of a true remote reference. The basic shape of these curves is a bell shape about the $45^{\circ}$ line. Additionally all sites show an upward kink at the very long periods. This indicates that these sites may be being effected by a relatively conductive feature in the lower crust.

As there is little variation in resistivity and basement is known to outcrop at the surface it is likely that there are very few layers below this group,. However these sites are being effected by the conductive feature in the lower crust that is seen in Groups $\mathrm{D}$ and $\mathrm{E}$.

\subsubsection{Conclusions}

The first conclusion is that the more resistive surface layer seen at most sites appears to be thicker near the centre of the volcano. This relative resistor is likely to be identifiable with volcanic debris. Below this layer and extending to the south and west is a shallow conductor. This conductor may be the Tertiary sediment identified in previous studies (Latter, 1981; Sissons and Dibble, 1981), although wet, altered volcanic debris can have similar resistivity signatures. Below this a resistive layer consistent with greywacke basement is present.

A minimum in TE apparent resistivity appears in long period data at sites east of the volcano. It is possible that this is also present below sites MTR-117a and MTR118a although the minimum is not as distinctive. The three sites measured by Hanekop (2002) around the volcano also showed this distinctive minimum. This conductive feature may be associated with the volcanic system or the regional structure of the North Island. 
In order to put constraints on the structure and establish if the above interpretations are correct the data need to be modelled. Prior to this, it is necessary for the dimensionality of the data to be analyzed to determine the most appropriate modelling technique. Dimensionality analysis in this survey has been conducted through the use of the phase tensor method.

\subsection{Phase Tensor Ellipses}

The phase tensor ellipses show the response to conductivity features influencing the electric and magnetic fields at each frequency separately. These influencing features can often be identified by the spatial representation of the ellipses. When combined with the parameters of ellipticity, $\beta$ and strike, areas that can be appropriately modelled by 1 or 2-D inversions can be identified.

When the phase tensor ellipses are plotted on a map of the study area the direction of the major axes points in the preferred direction of current flow. Comparing the maps for different frequencies clearly shows how the influencing structures vary with frequency. In order to discuss how the phase tensor ellipses vary, the maps from each of the forty frequencies have been split into three categories; high frequency maps (representing frequencies $>10 \mathrm{~Hz}$ ), dead band frequencies maps (representing frequencies between $10-0.1 \mathrm{~Hz}$ ), and long period maps (representing frequencies $<0.1 \mathrm{~Hz}$ ). Maps of the phase tensor ellipses at every fourth frequency measured are presented in appendix B.

\subsubsection{High Frequency Phase Tensor Ellipses}

High frequency data represent the response to the near surface structure. The phase tensor ellipse maps examined in this section represent frequencies between 360 and $10 \mathrm{~Hz}$, which corresponds to the top $\approx 500 \mathrm{~m}$ (if an average resistivity of $10 \Omega \mathrm{m}$ is assumed) of the structure. Figure 4.11 shows the phase tensor ellipse map representing the ellipses at a frequency of $240 \mathrm{~Hz}$ as an example for this section. Within this depth range the lithology is a mixture of volcanic tephra and lava (shown in seismic studies by 
Olson (1985); Latter (1981); Sissons and Dibble (1981); and in MT studies by Marriott (1969); and in various geological studies).

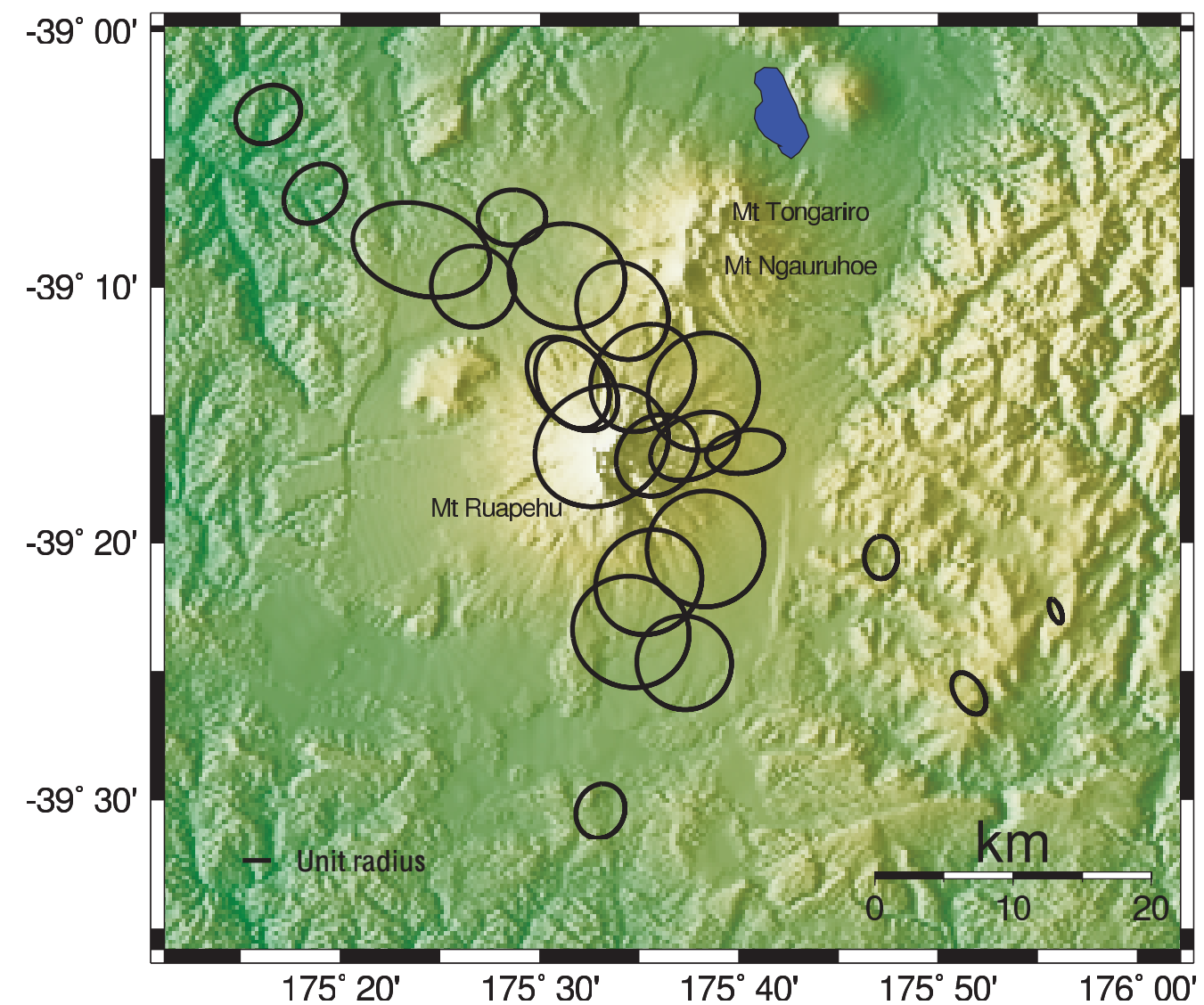

Figure 4.11 The phase tensor ellipse map for a frequency of $240 \mathrm{~Hz}$, this is representative of the other high frequency phase tensor ellipse maps.

From the unit scale at the bottom left of figure 4.11 it can be seen that most of the circles are larger than unity, indicating phase values of $>45^{\circ}$. This implies that within this period range resistivity is decreasing with depth. Additionally, the ellipticity at most sites is small indicating that the structures influencing the electric field do not have a dominant direction - implying that the structure is 1-D.

The sites that do show large ellipticity coincide with sites located near large topographic changes. Figure 4.12 shows an enlargement of the ellipses associated with sites MTR-107a and MTR-110a overlying a contour map. This figure shows that the long axes of the phase tensor ellipse points in the downhill direction approximately following the axes of the ridges. As free air has a much higher resistivity than the surface 
rock material, the topographical surface forms a significant boundary with which the major axis of the ellipses align. This illustrates that high frequency data are affected by topography. Furthermore it is reasonable to assume that the greater the consistency in the topographic strike the greater the ellipticity of the phase tensor will be.

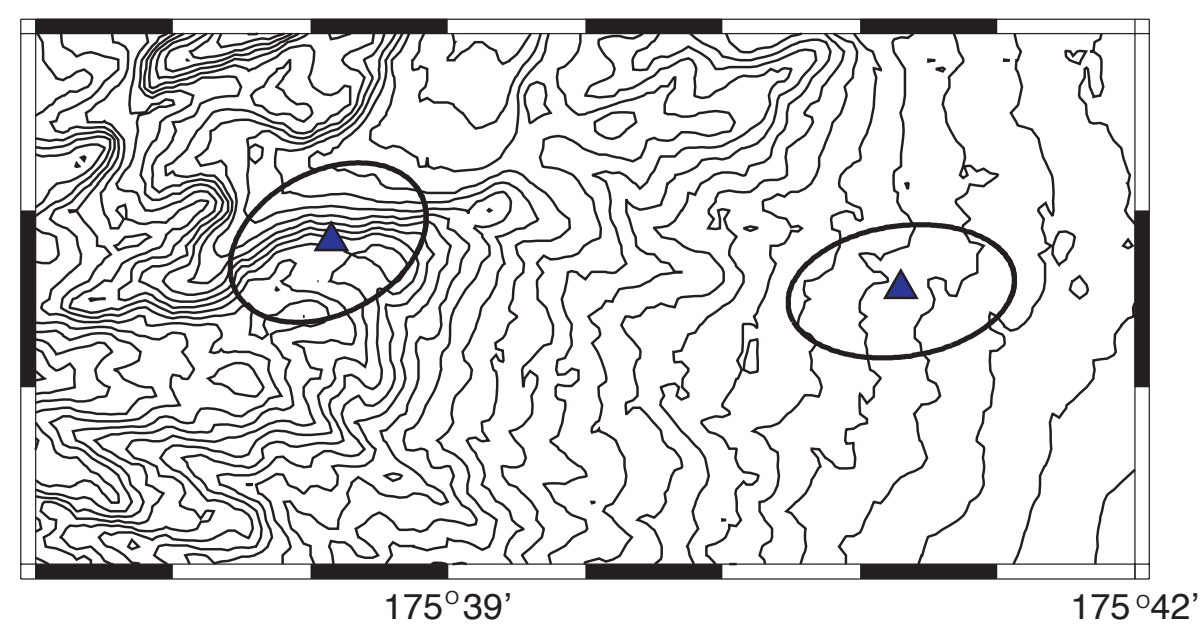

Figure 4.12 The effects of topography on high frequency phase tensor ellipses. The ellipses represent the phase tensor ellipses at $240 \mathrm{~Hz}$ for sites MTR-107a and MTR-110a superimposed onto a contour map.

From the discussion above we can see that the data are not free of inductive distortion. The phase tensor distortion removal method only removes galvanic distortion. Thus using the ellipse plots to identify the frequencies and sites effected by inductive distortion means that these can either be removed from the inversion input file or the topography can be included to create realistic models.

While local topography only affected the very high frequencies $(>100 \mathrm{~Hz}$ ), there are a number of other common features in the phase tensor ellipses at high frequency. These are:

- There is no dominant direction of major axes.

- Through the first five frequencies all of the ellipses increase in size, indicating decreasing resistivity with depth.

- Through the next ten frequencies ellipses at the sites on the volcano continue to be large. 
- The further away from the volcano a site is located the quicker the size of the ellipse decreases to below unity (i.e. where resistivity is increasing with depth).

- The sites to the east (Group F in section 4.2) do not follow this pattern. These sites have small ellipses, with high ellipticity that indicate that resistivity is increasing with depth in a non 1-D environment right from the highest frequency mapped. Topographic maps do not indicate that this is solely due to topographic features.

- West of the Waimarino Fault (presumed to be on the Tertiary sediment) there is a very consistent pattern between sites, with the long axes of the ellipses in the same direction despite the rough nature of the local topography. This implies that even at shallow depths, the same electrical structure is effecting these sites.

Overviews of the high frequency data are that although some sites are effected by local topography, west of the Desert Road Fault the data are predominantly 1-D at frequencies above $10 \mathrm{~Hz}$. The large ellipses at the very high frequencies indicate that the resistivity is decreasing with depth. This is consistent with the underlying relative conductor identified from the apparent resistivity curves described in the previous section. The ellipse size is reduced to below unity at higher frequencies for sites further away from the centre of the volcano. Therefore this conductor is probably thickest directly below the volcano. Once the ellipses are below unity size the underlying resistivity is increasing with depth. The lowest frequencies within this range $(\approx 10$ $H z)$ show that the resistivity is increasing with depth.

\subsubsection{Dead Band Phase Tensor Ellipses}

Within the dead band $(10-0.1 \mathrm{~Hz})$ there is a lower signal to noise ratio, therefore data within this frequency range tend to have larger uncertainties. The first thing to note within this frequency range is that many of the ellipses are much smaller than the high frequency ellipses and in many cases are smaller than unity. In figure 4.13 the phase tensor ellipses have a smaller radius and greater ellipticity than in figure 4.11. This indicates that a higher resistivity layer underlies the relative conductor seen above. 


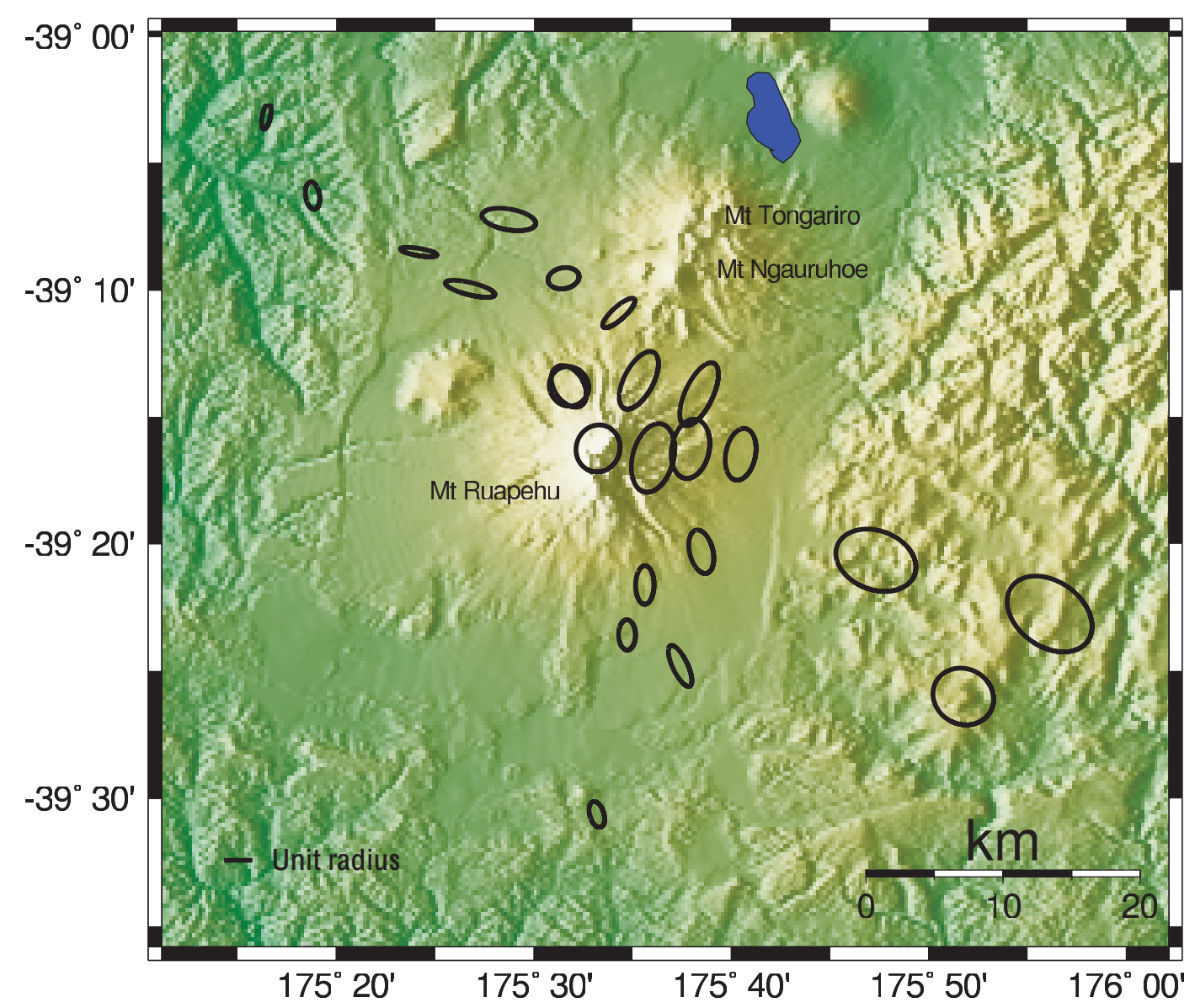

Figure 4.13 The phase tensor ellipse map for a frequency of $1.125 \mathrm{~Hz}$, this is representative of the other dead band phase tensor ellipse maps.

Below frequencies of $1.125 \mathrm{~Hz}$ the 1-D circles are replaced by ellipses. On opposing sides of the TVC boundary the long axes of the ellipses point in orthogonal directions. The major axes for the ellipses representing the eastern sites (on greywacke) point towards the TVC whereas those for adjacent sites within the TVC roughly align with the eastern boundary. At the western boundary the major axes of the ellipses representing the sites outside the TVC point towards the boundary while those of sites west of the boundary are aligned parallel to it. This is consistent with the common resistivity values assigned to the recognized surface lithologies and the standard observation that on the conductive side of a boundary the preferred electric field orientation is parallel to the boundary, while on the resistive side it is perpendicular to that boundary.

For the sites between the TVC boundaries there is much less uniformity of the major axes directions of the phase tensor ellipses. As the frequency decreases many of 
the ellipses have a major axes roughly radial to the volcano, indicating that the volcano is influencing data collected within this band. Radial alignment with Mt. Tongariro is also seen in some sites to the north (MTR-113a, MTR-114a and possibly MTR-112a). However, site MTR-117a at the summit still appears to be 1-D and all other signals are likely to be obscured by the effects of nearby Crater Lake.

Towards the end of the dead band $0.3 \mathrm{~Hz}$ (or period of $3.3 \mathrm{~s}$ ) the major axes start to line up in a north-northeast direction. Despite the variations in the degree of ellipticity and the parameters indicating that the structure is $3-\mathrm{D}$, all sites now seem to be effected by a single feature. This alignment is in broad agreement with the regional strike of the area. Most geological features in the eastern North Island have a predominantly north-northeast strike and any of these features could be the cause of this alignment.

\subsubsection{Long Period Phase Tensor Ellipses}

Within the ellipses representing the long period data there is a constant uniform alignment of the major axes at each site. Although the direction of the alignment changes with frequency the uniformity does not. At the shortest periods in this group the major axes of the phase tensor ellipses are aligned in the north-northeast direction identified above. As the period increases the major axes rotate to the southeast. Figure 4.14 shows the alignment of the ellipses at $230 s$ where they are pointing to the southeast.

Uniform directions of the major axes of phase tensor ellipses are recorded from 15 $s$ to $1000 s$. The $90^{\circ}$ clockwise rotation of the major axes occurs in the period range of $15-150 \mathrm{~s}$. A simplified skin depth calculation indicates that these frequencies are associated with depths of approximately $140 \mathrm{~km}$, deep enough to be influenced by the east coast of New Zealand or the Pacific/Australian plate boundary which is present just to the east of the North Island.

The southeast alignment is consistent with the preferred flow directions indicated by anisotropy, both electrical (Hanekop, 2002), and seismic (Gerst and Savage, 2004; Miller and Savage, 2001), within the area. The physical causes of electrical and seismic anisotropy differ and anisotropic directions often do not coincide. Although it is possible that this alignment is due to anisotropy it is not required in the interpretation of 


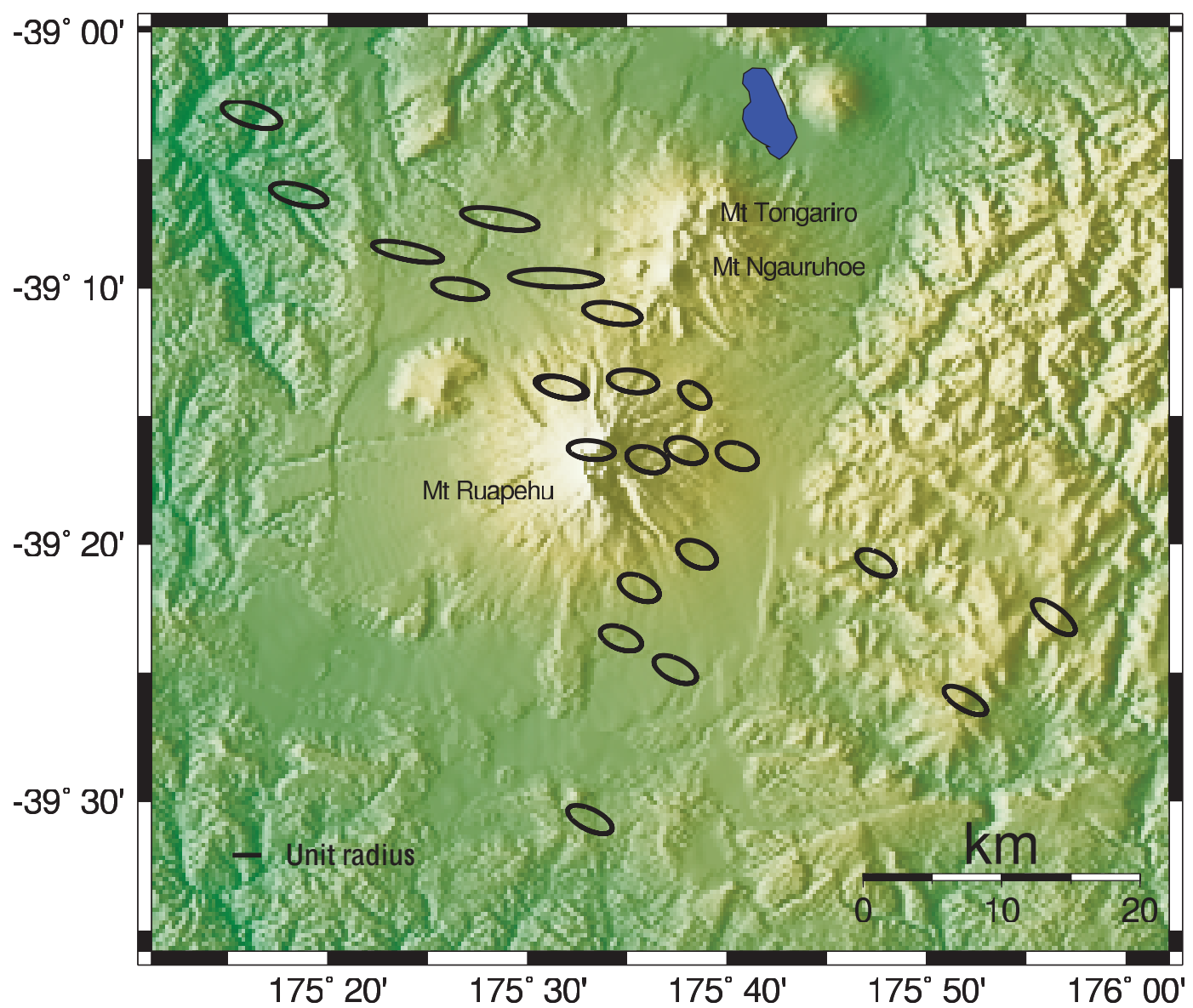

Figure 4.14 The phase tensor ellipse map for a period of $230 \mathrm{~s}$, this is representative of the other long period phase tensor ellipse maps.

these ellipses.

The uniformity of the ellipses disappears at the longest periods recorded. The ellipticity at some sites is extreme. This is probably a result of the fact that at these very long periods there are less cycles to average into each data point and therefore, they are more susceptible to outliers.

The dimensionality parameters indicate that much of the long period data are 2D. The most probable structure causing this 2-D response is the subduction zone or land/sea boundary to the east. However a 2-D inversion of the entire data set is unlikely to be realistic due to the $3-\mathrm{D}$ nature of the data at higher frequencies which prevent surface constraints on any 2-D structure at these depths. 


\subsubsection{Conclusions}

The phase tensor ellipses have identified the main influencing features within the area. These are summarized as being:

- Much of the near surface structure is 1-D.

- Local topography has a strong influence on frequencies greater than $100 \mathrm{~Hz}$.

- At frequencies of $15 \mathrm{~Hz}$ data are effected by local structures.

- Large scale topography is still affecting data within the dead band (i.e. the volcano).

- Inductive distortions are apparent down to $5 \mathrm{~s}$.

- At a period of $15 s$ all sites start to be influenced by the regional strike of the area.

- Long period data are strongly influenced by 2-D tectonic structure.

From the analysis of the dimensionality and phase tensor ellipses it can be seen that in order to obtain a realistic structure of Mt. Ruapehu 3-D modelling will be required. In order to properly utilize a $3-\mathrm{D}$ inversion scheme this will also require more data to give a more uniform site distribution around the entire volcano.

Although the data should be modelled by a 3-D inversion scheme, a lack of data in critical areas prevents this from being feasible. For the purpose of placing some constraints on the structure the following initial modellings are deemed to be appropriate:

- 1-D models of the high frequency data to give a picture of the near surface resistivity structure.

- A 2-D model of the eastern boundary of the TVC where basement greywacke outcrops.

- A 2-D model of the western boundary of the TVC and the structure associated with the Waimarino Fault.

These models are discussed in the next chapter. 
CHAPTER 5

MODELLING

As identified in the previous chapter, limited 1 and 2-D modelling of parts of the data set may be appropriate. This chapter presents the models created and the interpretation of these models. As the 1-D sections of data were limited to the high frequency range, the determinant of the impedance tensor was used to derive full 1-D models as discussed below. Once again the models could be split into groups with similar characteristics. The following section then describes 2-D models for short transects that cross the TVC boundaries. Figure 5.1 show the 2-D profiles used in the modelling, as well as the boundaries for the 1-D model groupings. When the results are compared a basic resistivity structure for the volcano begins to emerge. This structure was then used in a 3-D forward model to test its validity.

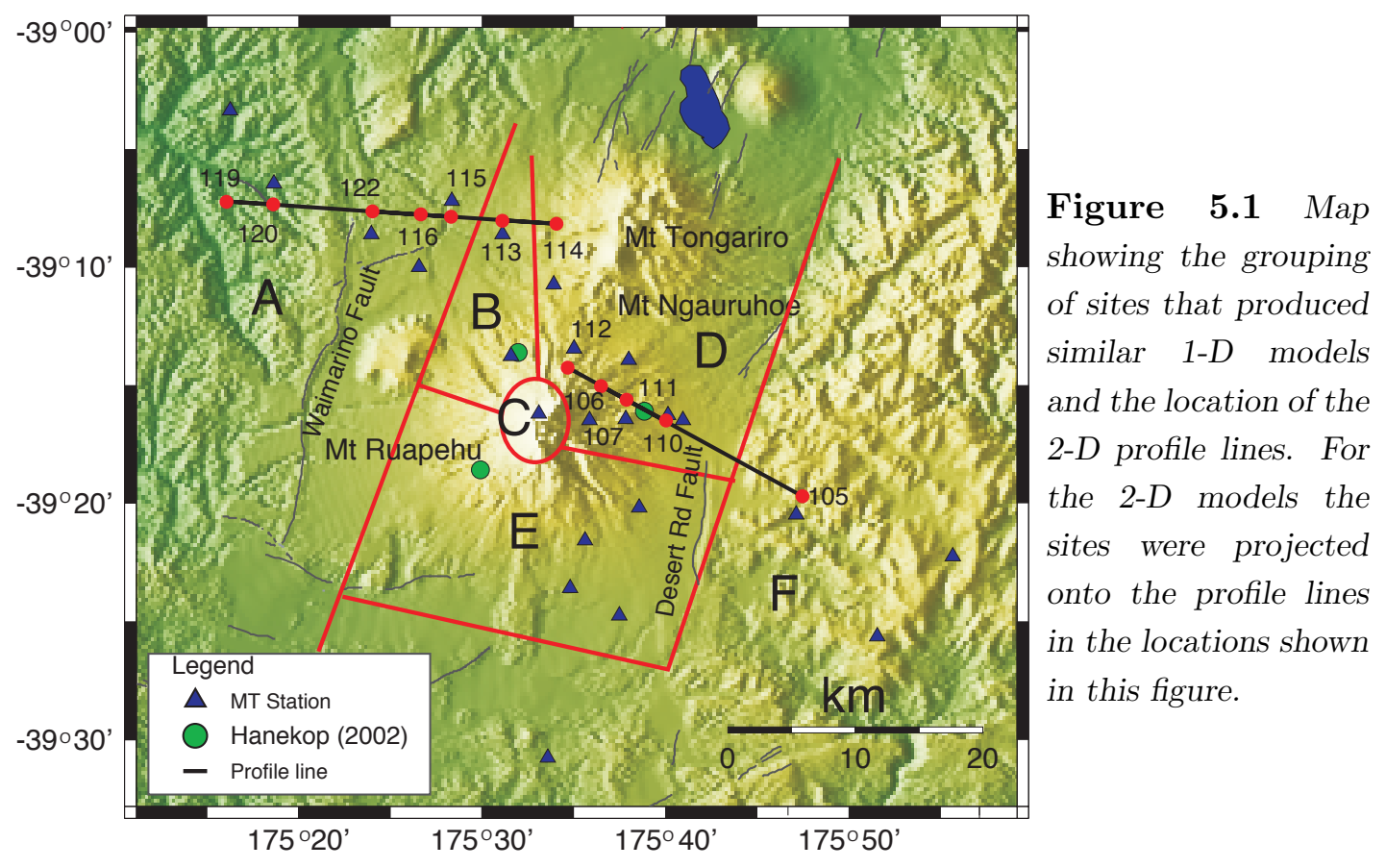




\section{$5.1 \quad 1-D$ Modelling}

Previous phase tensor analysis showed that a very limited portion of the data is strictly 1-D. The majority of data are 3-D throughout most measured frequencies, with significant differences between the polarisations in both apparent resistivity and phase data. As a result, 1-D modelling over the entire frequency range has been based on apparent resistivity and phase curves calculated from the determinant impedance, defined by the square root of the determinant of the impedance tensor. The determinant impedance was first used by Ranganayaki (1984) and is invariant with respect to the co-ordinate axes regardless of the dimensionality of the data.

Modelling studies suggest that 1-D modelling of the determinant impedance is indicative of layering beneath a measurement site even in non 1-D situations (Ingham, 2003, pers.comm.). However, these models are only indicative of possible layering and values given should not be deemed fixed. In 1-D modelling the structure is solved for the conductance, the product of conductivity and thickness. This calculation is the cause of the non-uniqueness in the any models and may be the cause of the variations between models created for individual sites. The determinant apparent resistivity and phase were modelled using the IPI_MT code (Bobachev, 1990).

The 1-D models have been split into six groups with common characteristics, as shown in figure 5.1. The characteristics of each group are described individually prior to a discussion of the entire set of 1-D models. For each of the models two figures are presented:

1. The log of resistivity plotted against the log of depth below the surface; referred to as the depth plot.

2. The log of resistivity plotted against the altitude down to five kilometres, corrected for site elevation; referred to as the altitude plot.

Theoretical data were produced from forward modelling the models presented here, and the data was then compared to the determinant impedance to see how well the data fitted. For the complete set of model fits refer to appendix C. 


\subsubsection{Group A}

Group A consists of the five sites in the northwest, grouped because of their proximity to the Waimarino Fault. These are the first five sites from the left in the 2-D model labelled Profile A. The first feature identified from the depth plot (5.2) is the large
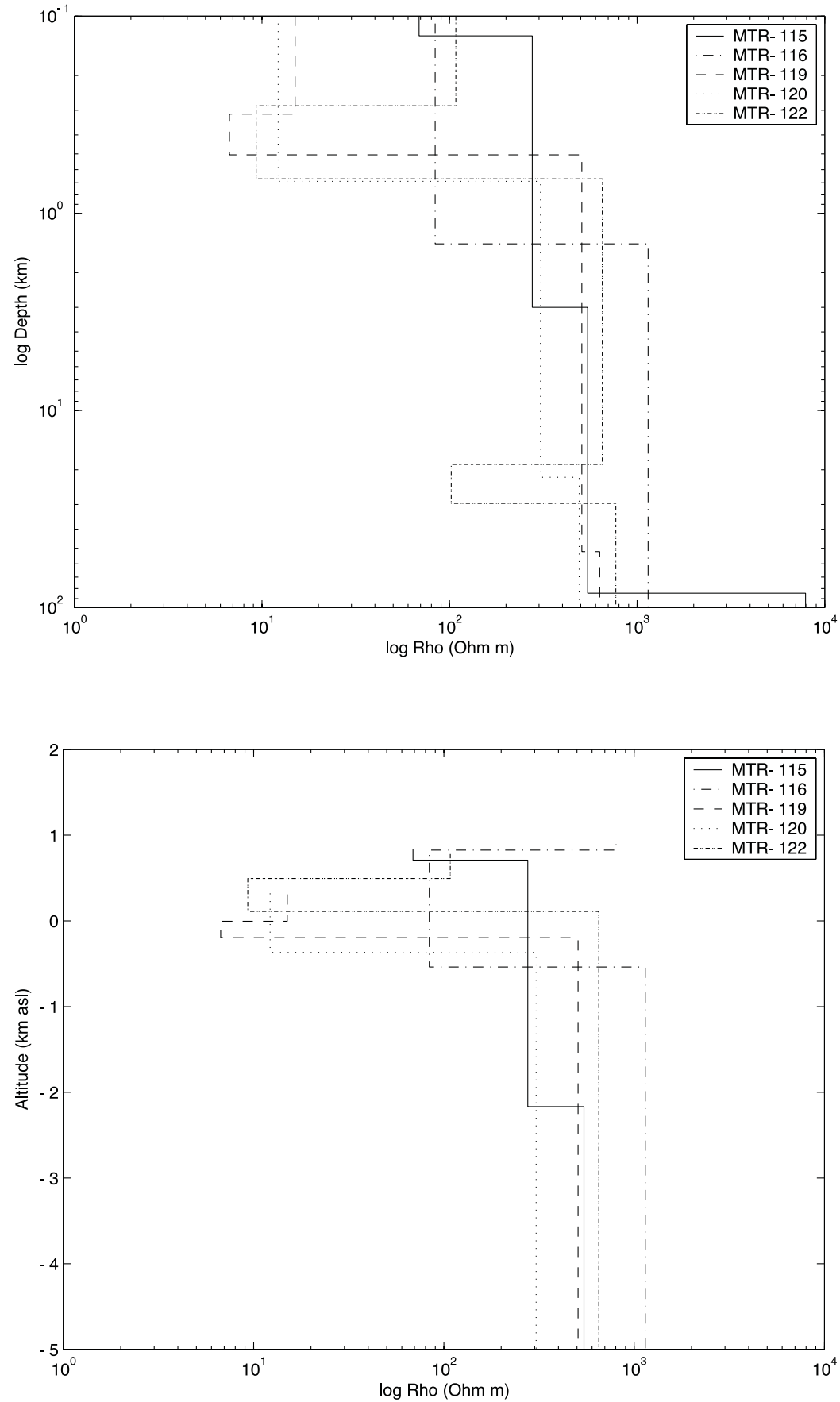

Figure $\mathbf{5 . 2}$ Depth plot of 1-D models produced in group A. Sites MTR-115a, MTR-116a, MTR119a, MTR-120a and MTR-122a.

Figure 5.3 Altitude plot showing near surface structure from 1-D models produced in group A. Sites MTR-115a, MTR-116a, MTR119a, MTR-120a and MTR-122a.

range of surface resistivities, ranging from 10-800 $\Omega \mathrm{m}$. Due to this large range, the sites do not appear to have much in common, although when compared in the altitude 
plot (5.3) the sites appear more coherent. The considerable elevation difference between these sites accounts for some of the differences although the model from site MTR-115a is sufficiently different to warrant an individual description.

In general it is observed that the higher the elevation of the site, the higher the surface resistivity. Apart from site MTR-115a, the surface material is then underlain by a relative conductor although the resistivity and thickness do vary between sites. Resistivities of the conductive layer modelled here have a range from $8-80 \Omega m$, and thickness from 200-1500 m, which give approximately similar conductance values. The mean value of resistivity of $\approx 10 \Omega m$ is compatible with the existence of Tertiary sediments or wet, altered volcanic debris.

The two sites on the west of the Waimarino Fault (MTR-119a and MTR-120a) have low surface resistivities, missing the higher resistivity surface layer that is present on the other side of the Fault. The surface layer here is consistent with the shallow conductive layer seen east of the fault and may be the displaced continuation of the shallow conductor.

Below the shallow/surface relative conductor there is a uniform layer with a resistivity value between 300 and $1000 \Omega m$. This layer is approximately continuous down to $100 \mathrm{~km}$, with the exception of a second relative conductor recorded at $\approx 20 \mathrm{~km}$ deep below site MTR-122a. This second conductor exhibits similar characteristics to the second conductor indicated in models from the upper northeastern flank (and is discussed in Group D). This conductor may be related to the long period minimum in apparent resistivity identified in the curves from some of the sites in Group E discussed in section 4.2.5.

The model produced from site MTR-115a does not conform to the other models. Instead of the shallow conductor identified in each group, site MTR-115a shows a series of steps gradually increasing in resistivity. The four steps increase resistivity from 700 to $7000 \Omega m$ in the top $100 \mathrm{~km}$ of the Earth.

In summary: Group A shows how the models vary across the Waimarino Fault.

- In the east there is a surface resistor overlaying a conductor, 
- to the west is the conductor forms the surface layer. Although there is the possibility that the conductors are not in fact related due to the presence of the Waimarino Fault,

- beneath this conductor the models indicate the presence of a relatively homogeneous resistive basement layer,

- a second deeper conductor was identified beneath one site,

- and site MTR-115a does not match this identified stratigraphy.

\subsubsection{Group B}

Group B is composed of the two sites on the upper northwestern flank, MTR-113a and MTR-118b. MTR-114a which was also in the original Group B has been shifted to Group D for this section. The ' $b$ ' set of the data collected from site MTR-118 is presented here as the model obtained was considered to be more realistic than that obtained from MTR-118a (see section 4.1 for the difference between sets). Each of

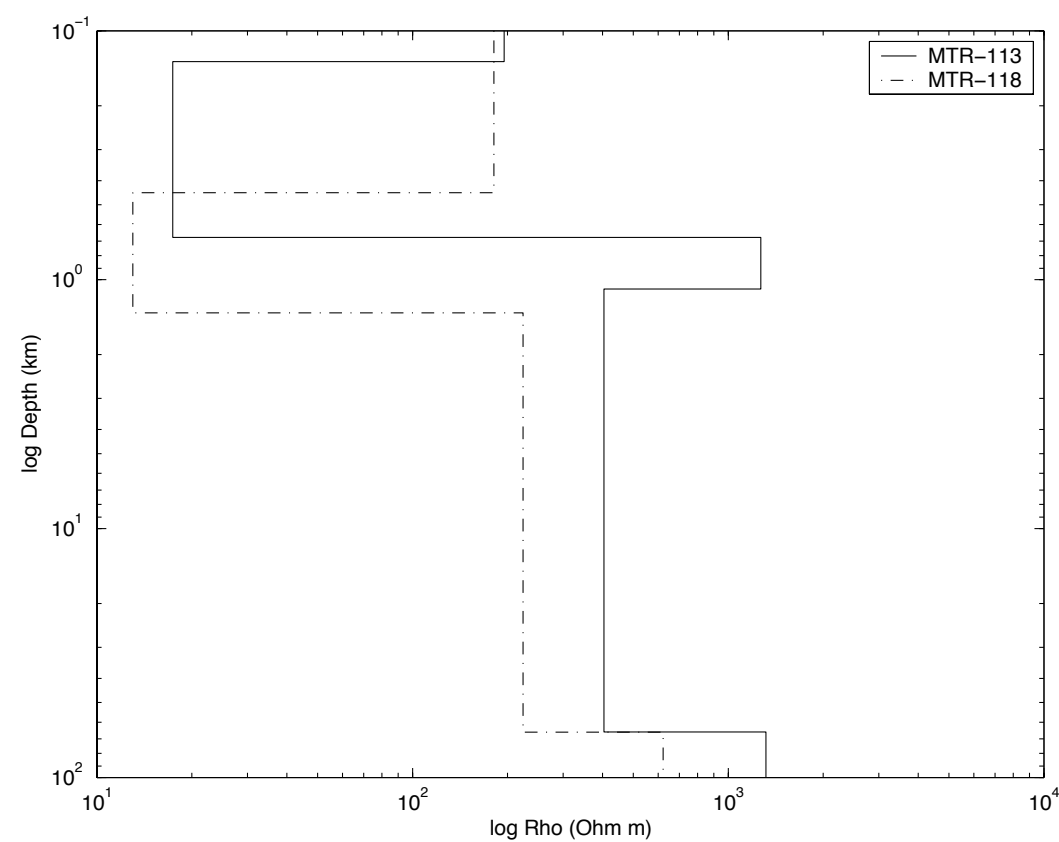

Figure 5.4 Depth plot of 1-D models produced in group $\mathbf{B}$. Sites MTR-113a and MTR-118b.

these sites shows evidence of a single shallow conductor in the depth plot (5.4). From the altitude plot (5.5) it can be seen that the shallow layers below both sites are similar. 


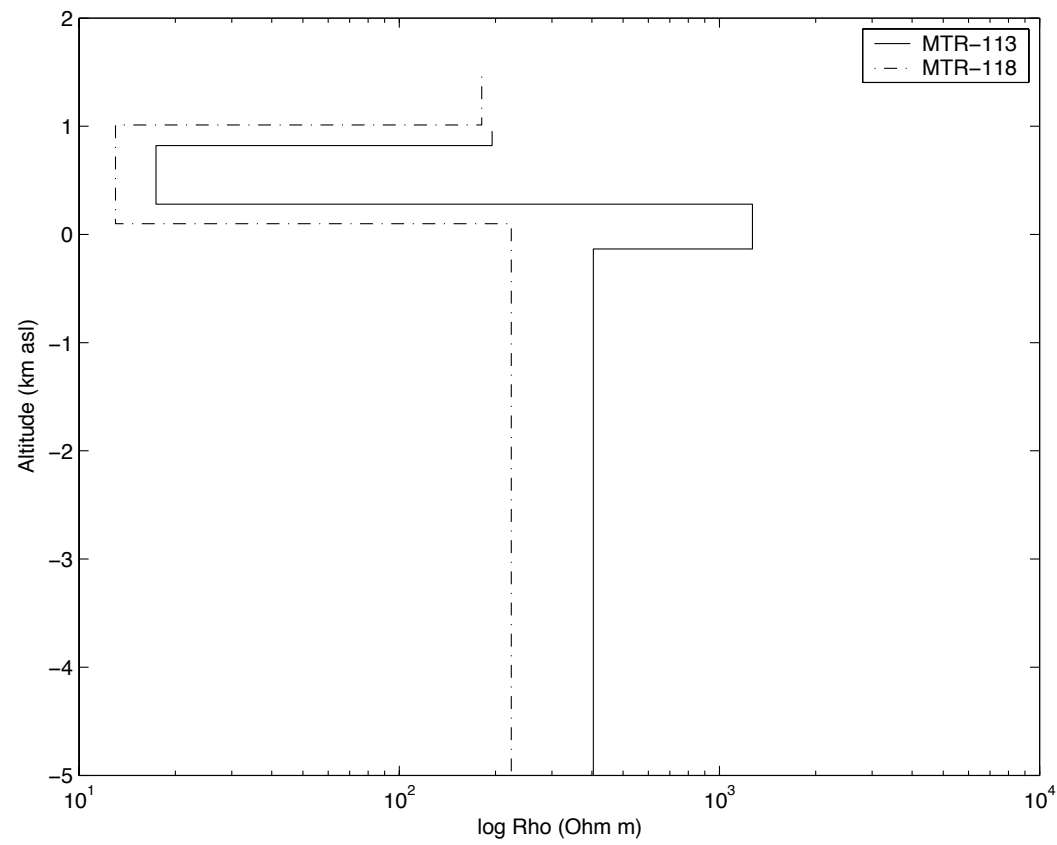

Figure 5.5 Altitude plot showing near surface structure from 1 $D$ models produced in group B. Sites MTR113a and MTR-118b.

A thin (200 $m$ thick) layer of $200 \Omega m$ overlies a $20 \Omega m$ conductive layer. The top of this conductor is just over $800 \mathrm{~m}$ (asl) high.

Below this shallow conductor site MTR-113a shows evidence of a $400 \mathrm{~m}$ thick resistor. A resistive layer of this nature is only seen beneath this site and site MTR104a (Group E). Beneath these surface features is a thick layer between $200-300 \Omega m$ that exists to $\approx 80 \mathrm{~km}$ deep. There is then a jump in resistivity to $\approx 1000 \Omega \mathrm{m}$ for both sites. There is no evidence in these models of the second deep conductive layer to be seen in following groups.

In summary: Group B shows evidence of four layers.

- A relatively resistive surface layer,

- a second conductive layer $(20 \Omega m$ ), that is less than $1 \mathrm{~km}$ thick,

- a thin extra layer exists in model MTR-113a, this layer has a resistivity of $\approx 2000$ $\Omega m$ and is $\approx 400 m$ thick,

- these are underlain by a $200-300 \Omega m$ layer down to $\approx 80 \mathrm{~km}$,

- below the last layer the resistivity jumps to $\approx 1000 \Omega m$. 


\subsubsection{Group C}

Group C consists solely of the site near the summit, MTR-117a. The depth plot (5.6)

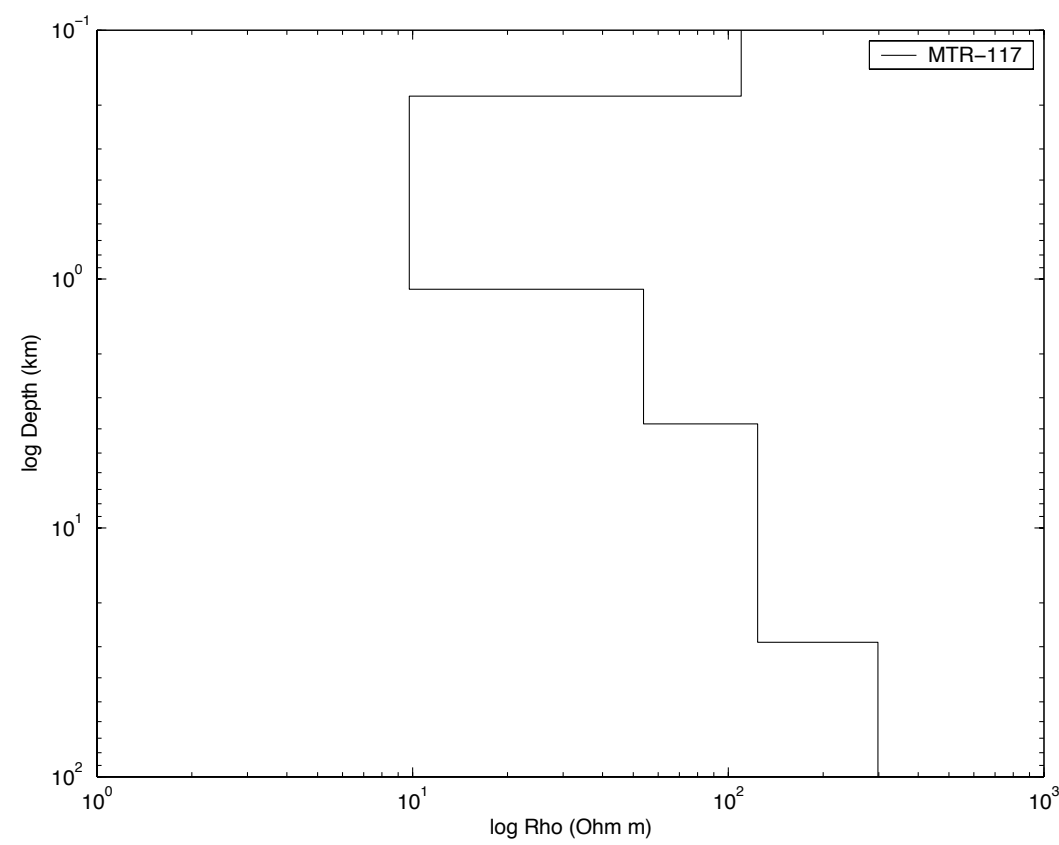

Figure 5.6 Depth plot of 1-D models produced in group $\mathbf{C}$. Site MTR-117a

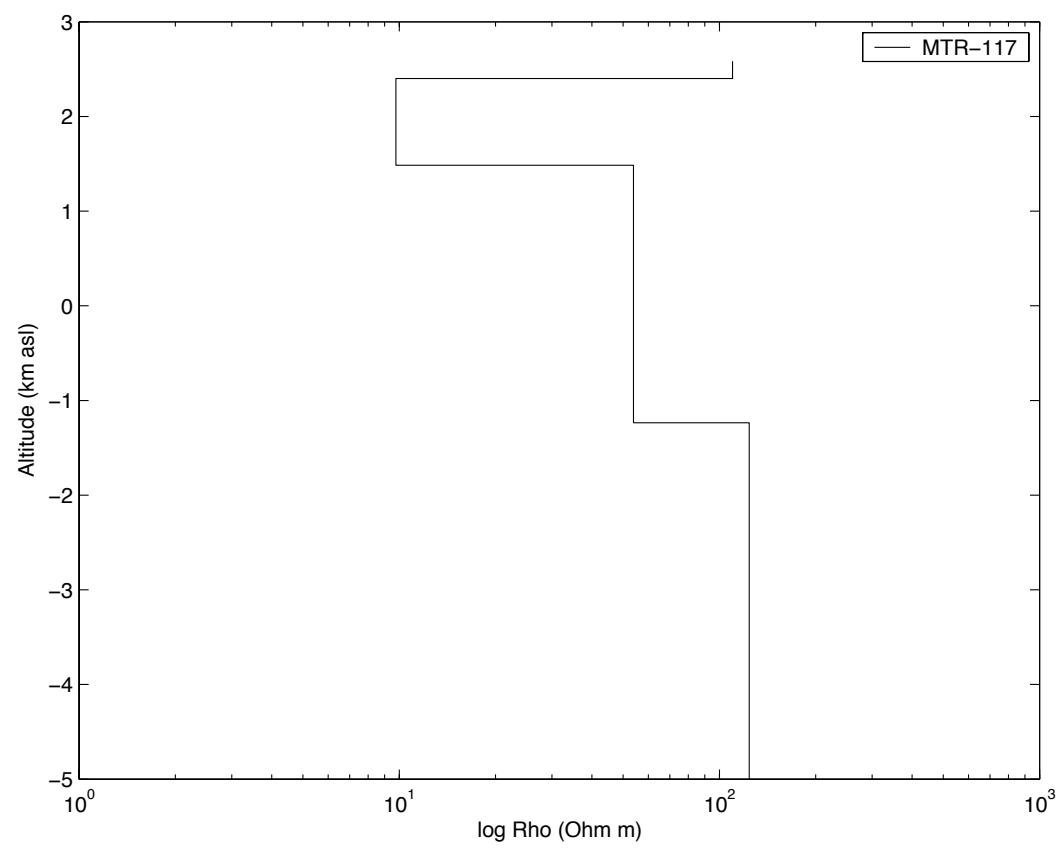

Figure 5.7 Altitude plot showing near surface structure from 1$D$ models produced in group C. Site MTR117 .

again shows evidence of a shallow conductor with a resistivity less than $10 \Omega m$ under a relatively resistive surface layer. The altitude plot (5.7) shows this conductor to be $1 \mathrm{~km}$ thick. From these plots it can be seen that the resistivity steps up in a series of layers that becomes increasingly more resistive. 
The depth plot (5.6) shows that below the surface conductor the resistivity increases in three steps, each cover half a decade and could be from a continuous layer that has gradually increasing resistivity with depth. The fifth and final layer in the top $100 \mathrm{~km}$ has a resistivity of $3000 \Omega m$.

Previous studies on volcanoes have indicated that magmatic material has a resistivity of $\approx 2 \Omega m$ which is much lower than any of the resistivities modelled below this site. This suggests that it is unlikely that hot molten magma exists in any large quantity beneath the centre of the volcano although the shallow conductor has a sufficient conductance to be associated with a hydrothermal system. The hot acidic lake in the crater is evidence of this system and is probably sufficient to explain the low resistivity measurements without the presence of a magma body.

In summary: The model from group $\mathrm{C}$ contained five layers.

- A relatively resistive surface layer,

- the second layer is conductive and has a resistivity less than $10 \Omega \mathrm{m}$ and is $1 \mathrm{~km}$ thick,

- below this there are three layers, increasing in resistivity by half an order of magnitude at each step, finally having a resistivity of $2000 \Omega \mathrm{m}$ at $100 \mathrm{~km}$ below the surface.

\subsubsection{Group D}

Group D contains the six sites on the northeastern upper flank. The depth plot (5.8) shows that there are two relative conductors, at depths of approximately 1 and $10 \mathrm{~km}$ below the surface. The altitude plot (5.9) shows that the upper of these varies between the sites. There is an order of magnitude difference in resistivity values between the surface layer and conductor in each model. The surface layer has resistivity values between 200 and $600 \Omega m$, dropping to between 10 and $30 \Omega m$ in the underlying layer.

The height to the top of the conductor is not as consistent in this group as it is in group E, varying between 300 and $1200 \mathrm{~m}$ (asl), although there is a correlation 


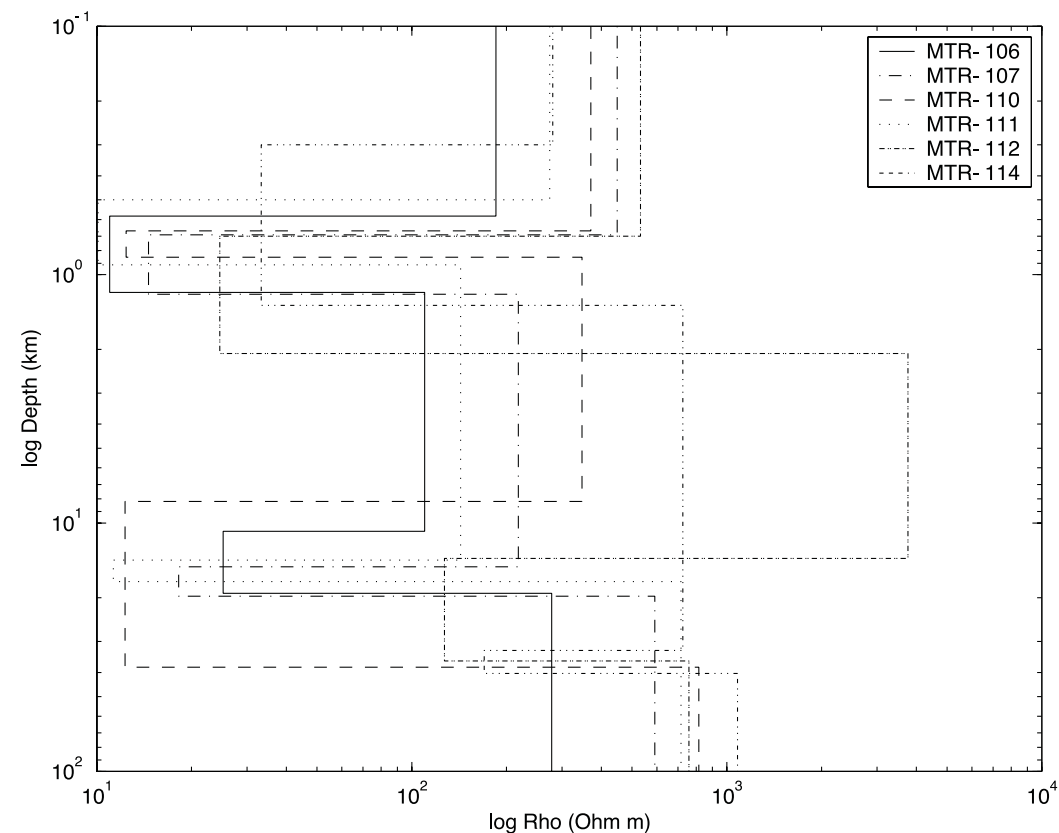

Figure 5.8 Depth plot of 1-D models produced in group D. Sites MTR-106a, MTR-107a, MTR110a, MTR-111a, MTR-112a and MTR-114a.

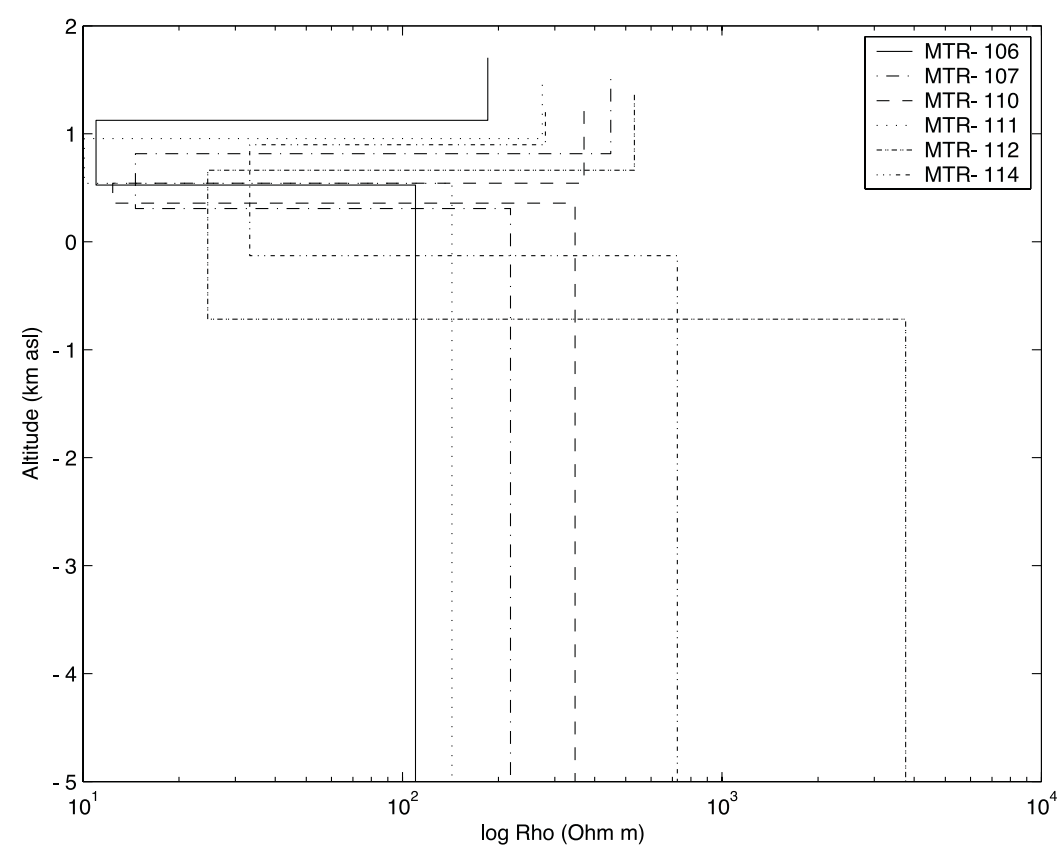

Figure 5.9 Altitude plot showing near surface structure from 1-D models produced in group D. Sites MTR-106a, MTR107a, MTR-110a, MTR-111a, MTR$112 \mathrm{a}$ and MTR-114a.

between the elevation of the site and the top of the conductor. The surface layer is $\approx 500 \mathrm{~m}$ thick in each model. The altitude plot (5.9) also shows that this conductive layer is twice as thick at sites MTR-112a and MTR-114a than at sites MTR-106a and MTR-111a. Although this last feature may just be an effect of the conductance calculation.

The resistivity of the third layer varies greatly between the models, ranging from $100-3000 \Omega m$. All models within this group show evidence of a second conductive 
layer in the $10-30 \mathrm{~km}$ depth range which correlates with the observed minimum in the apparent resistivity curves. A resistivity drop of more than half a decade is modelled for each site. However, a large range of resistivities were also estimated for this conductor. Below the fourth layer the resistivity values have a range of $200-1000$ $\Omega m$.

In summary: Group D produced models that contained five layers.

- A surface layer of $\approx 500 \Omega m$ resistivity and $\approx 500 m$ thick,

- this is underlain by a relative conductor of $10-30 \Omega m$ and less than $2 \mathrm{~km}$ thick,

- the third layer is more resistive although the resistivity is not well constrained,

- the fourth layer is a second conductor between $10-30 \mathrm{~km}$ deep,

- a resistive fifth layer having resistivity values between $200-1000 \Omega m$ completes the sequence.

\subsubsection{Group E}

Group E consists of the four sites occupied in Karioi Forest on the lower southeast flank. The depth plot (5.10) shows a uniform surface resistivity of $250 \Omega m$, and below this is a relative conductor of $10-30 \Omega m$. From the altitude plot (5.11) it can be seen that site elevation counts for much of the thickness difference in the surface layer. This conductive layer is approximately $800 \mathrm{~m}$ thick and first encountered at a height of $\approx 600 \mathrm{~m}(\mathrm{asl})$.

Below the relative conductor the resistivity increases to approximately $700 \Omega m$. The depth plot (5.10) shows a second relatively conductive layer roughly $10 \mathrm{~km}$ below two of the sites. The resistivity drops by more than half a decade. The sites that do not contain this conductor are those furthest away from the volcano. Below this second conductor resistivities range from $500-600 \Omega m$. The southern sites do show a jump from $600-2000 \Omega m$ around $70 \mathrm{~km}$ deep although this may be an artefact of the modelling. 


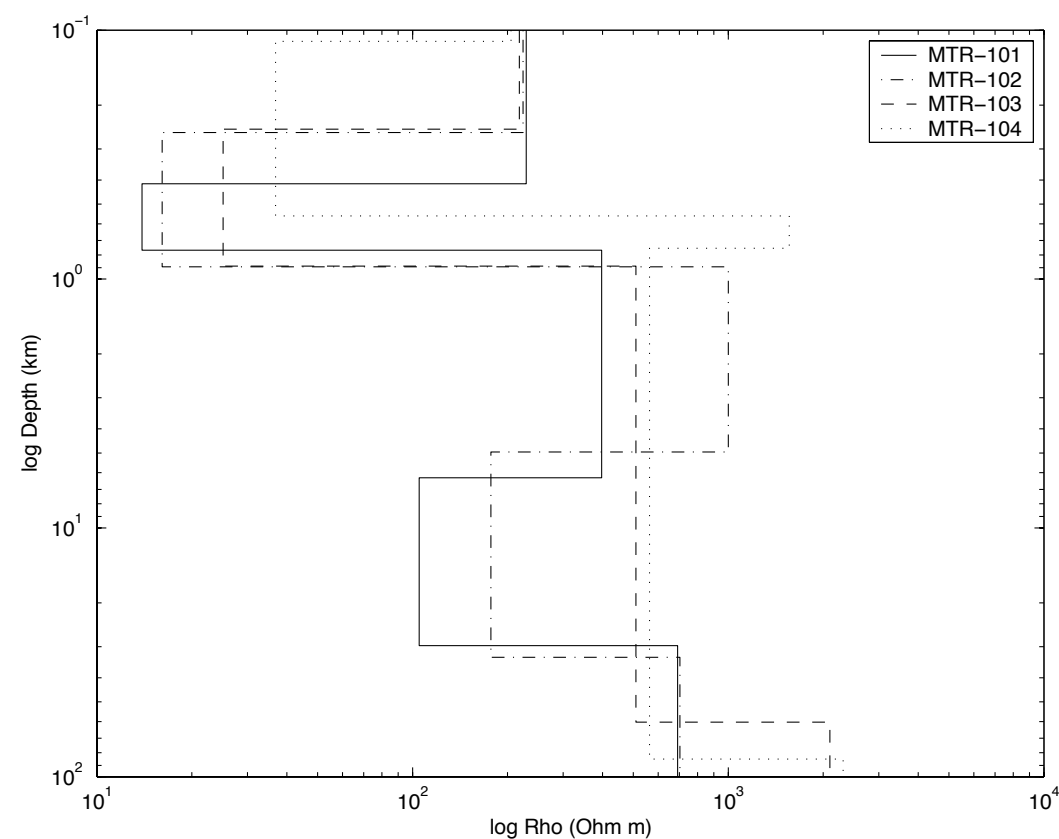

Figure 5.10 Depth plot of 1-D models produced in group E. Sites MTR-101a, MTR-102a, MTR103a and MTR-104a.

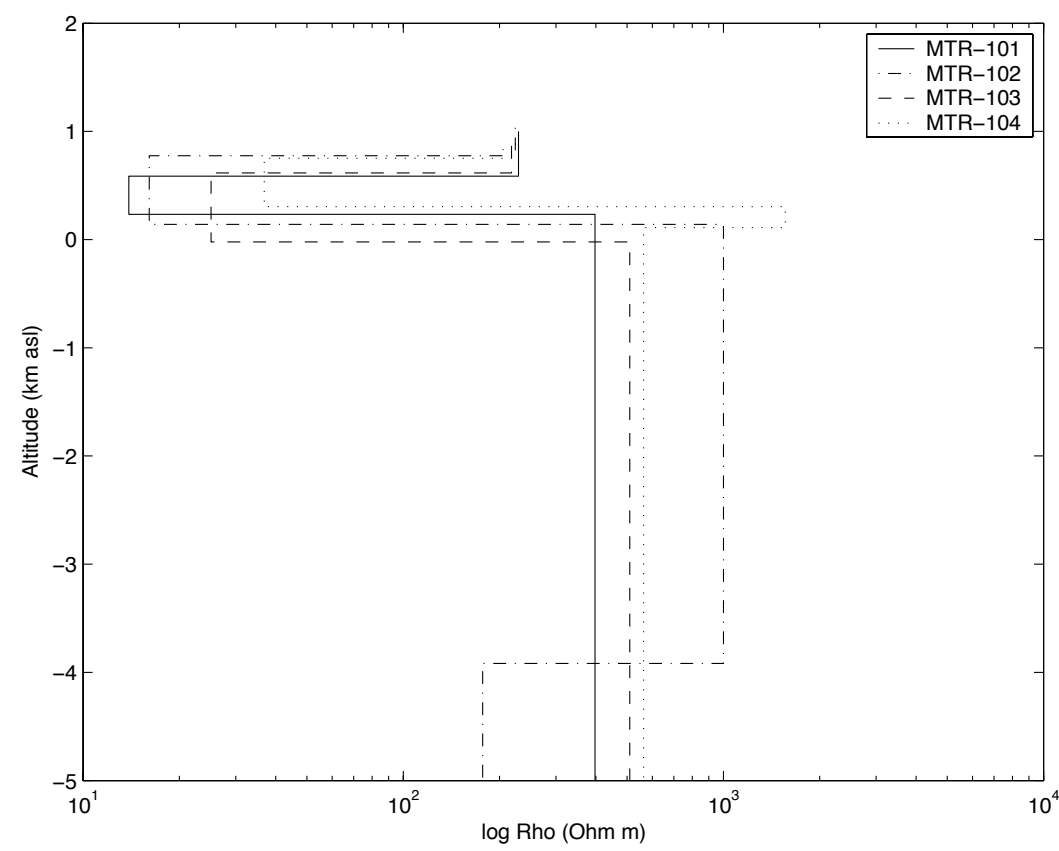

Figure $5.11 \quad A l-$ titude plot showing near surface structure from 1-D models produced in group E. Sites MTR-101a, MTR-102a, MTR103a and MTR-104a.

In summary: Group E produced models of four or five layers.

- A relatively resistive surface layer whose thickness depends on the elevation of the site,

- the second layer is a relative conductor $(10-30 \Omega m)$ approximately $800 m$ thick,

- this is underlain by a third more resistive layer averaging $700 \Omega m$, 
- the northern sites produced models that had a second conductive layer approximately $10 \mathrm{~km}$ deep,

- this second conductor is underlain by a layer of $500 \Omega m$,

- the models of the southern soundings do not contain the second conductive layer but have a much deeper boundary at $70 \mathrm{~km}$ deep where the resistivity increases to $2000 \Omega m$.

\subsubsection{Group F}

Group F contains the remaining sites to the south and east of the TVC. The first

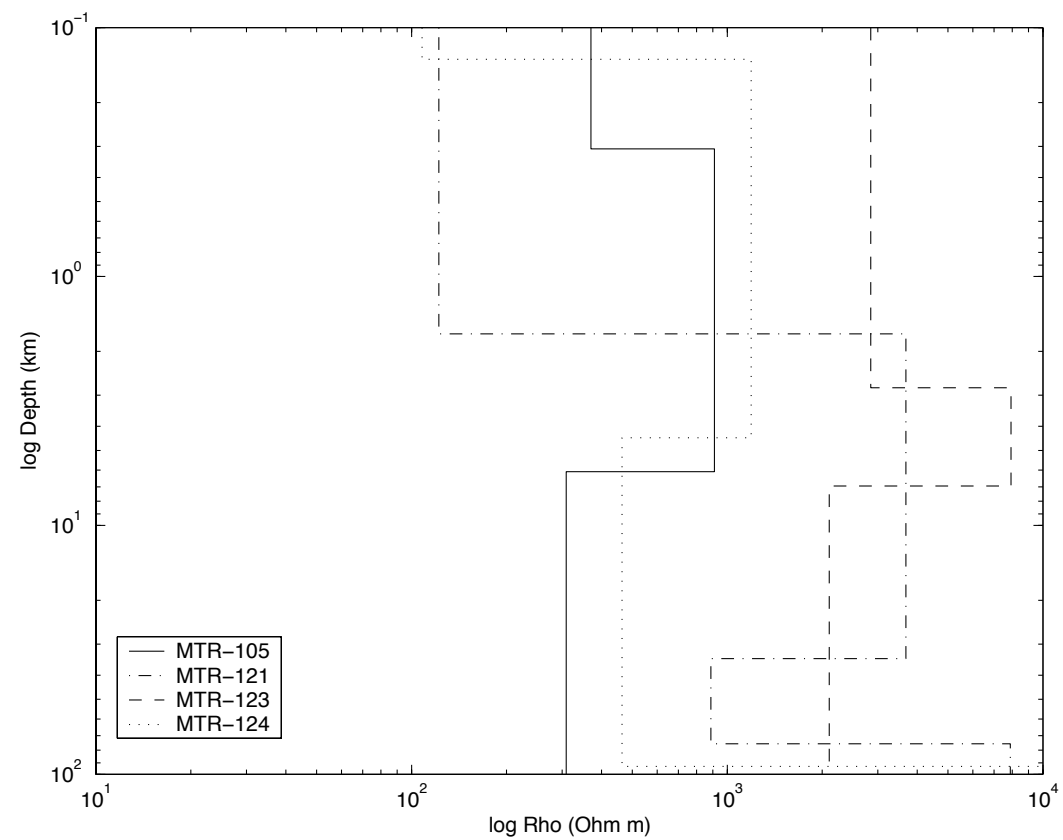

Figure 5.12 Depth plot of 1-D models produced in group F. Sites MTR-105a, MTR-121a, MTR123a, and MTR-124a.

feature to note in this group is that the model from site MTR-121a does not match the other models and will be discussed later separately. From the depth plot (5.12) it can be seen that there is a small resistivity range over the entire $100 \mathrm{~km}$ of the model. The model created from site MTR-123a shows a distinctly higher resistivity value modelled for all depths and appears to be statically shifted to the right when compared with the other curves.

Only site MTR-124a shows evidence of a relative surface conductor although the altitude plot (5.13) shows that this is only $100 \mathrm{~m}$ thick. Each of these three models 


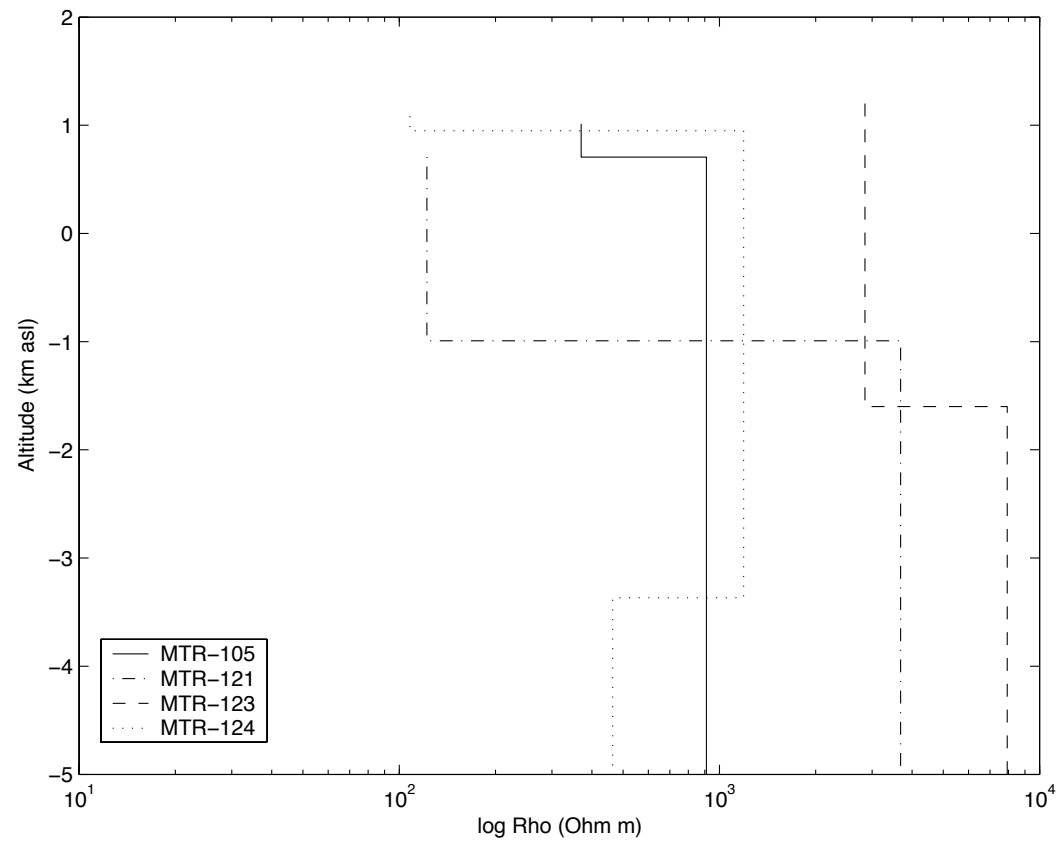

Figure $\quad 5.13 \quad A l-$ titude plot showing near surface structure from $1-D$ models produced in group F. Sites MTR-105a, MTR-121a, MTR123a, and MTR-124a.

could be fitted with only three layers, which indicates a simple structure. Each site shows a relative resistor at depth, although the top of the resistor varies by $2 \mathrm{~km}$. All sites show a decrease in resistivity at approximately $6 \mathrm{~km}$ deep. This decrease is of the order of half a decade giving resistivity values of $300-1000 \Omega m$.

The model created from site MTR-121a shows a distinct relative surface conductor similar to that seen west of the Waimarino Fault. This relative conductor is of the order of $1800 \mathrm{~m}$ thick and has a resistivity of $\approx 100 \Omega \mathrm{m}$. Below which the resistivity calculated is $2000 \Omega \mathrm{m}$. Again there is the presence of a second conductor at $20 \mathrm{~km}$ deep. Although this second relative conductor was not seen in the models from the two sites just north of site MTR-121a.

In summary: this group has two basic structures: that of the model from MTR121a, and that of the other models. However, in all cases the modelled resistivities are continuously high $(>100 \Omega m)$.

- The model from MTR-121a shows a relative surface conductor, and a secondary conductor at $20 \mathrm{~km}$ deep,

- meanwhile the other sites have a relatively homogeneous structure with an increase of half a decade in resistivity between 2 and $6 \mathrm{~km}$ deep. 


\subsubsection{Interpretation of 1-D models}

This subsection aims to bring all the above groups of models together to form a simple picture of the 3-D structure of the volcano.

Even between the separate groups it could be seen that there are a number of common features. The shallowest such feature is the presence of a resistive surface layer at sites within the TVC. The thickness of this layer generally varies with the elevation of the site, although the models from the upper flanks consistently show the layer to be $500 \mathrm{~m}$ thick. Resistivity values for this layer range from $100-400 \Omega \mathrm{m}$. This layer can be identified as young volcanic deposits. The uniform thickness of the layer on the upper flanks are consistent with the extent of recent lava flows.

Below the surface resistor within the TVC there is a shallow conductor. This conductor exhibits similar properties to the conductive surface layer modelled to the west and south of the TVC. It has a resistivity of $10-30 \Omega m$ and extends to just below sea level. There are two main candidates for the lithology: wet or altered volcanic debris or Tertiary sediment. To test which lithology best suits the properties of the conductor, the shape of the top surface of this conductor was investigated. A plot was created that showed the altitude of the site and altitude to the top of the conductor plotted against horizontal distance from the summit site (figure 5.14). This was done under the assumption that if it was volcanic in origin the height of the top of the conductor would decrease with distance from the center of the volcano.

It can be seen from figure 5.14 that the layer has a uniform thickness from 600 to $1000 \mathrm{~m}$ (asl) beneath the volcano. The only exception to this observation is the site occupied near the Crater Lake (MTR-117a). Ignoring site MTR-117a, the altitude of the top surface of the conductor was mapped. Figure 5.15 shows that even with the exclusion of the site at the Crater there is still a doming effect around the higher sites. This implies that this conductor is in some way related to the formation of the volcano. The sites outside the TVC were not included in the map as the top of the conductor at these sites is the erosional ground surface and can be identified with Tertiary sediment and is not associated with the volcano. At the sites further from the Crater it can be seen from figure 5.15 that the doming effect is reduced, and this may be where the 


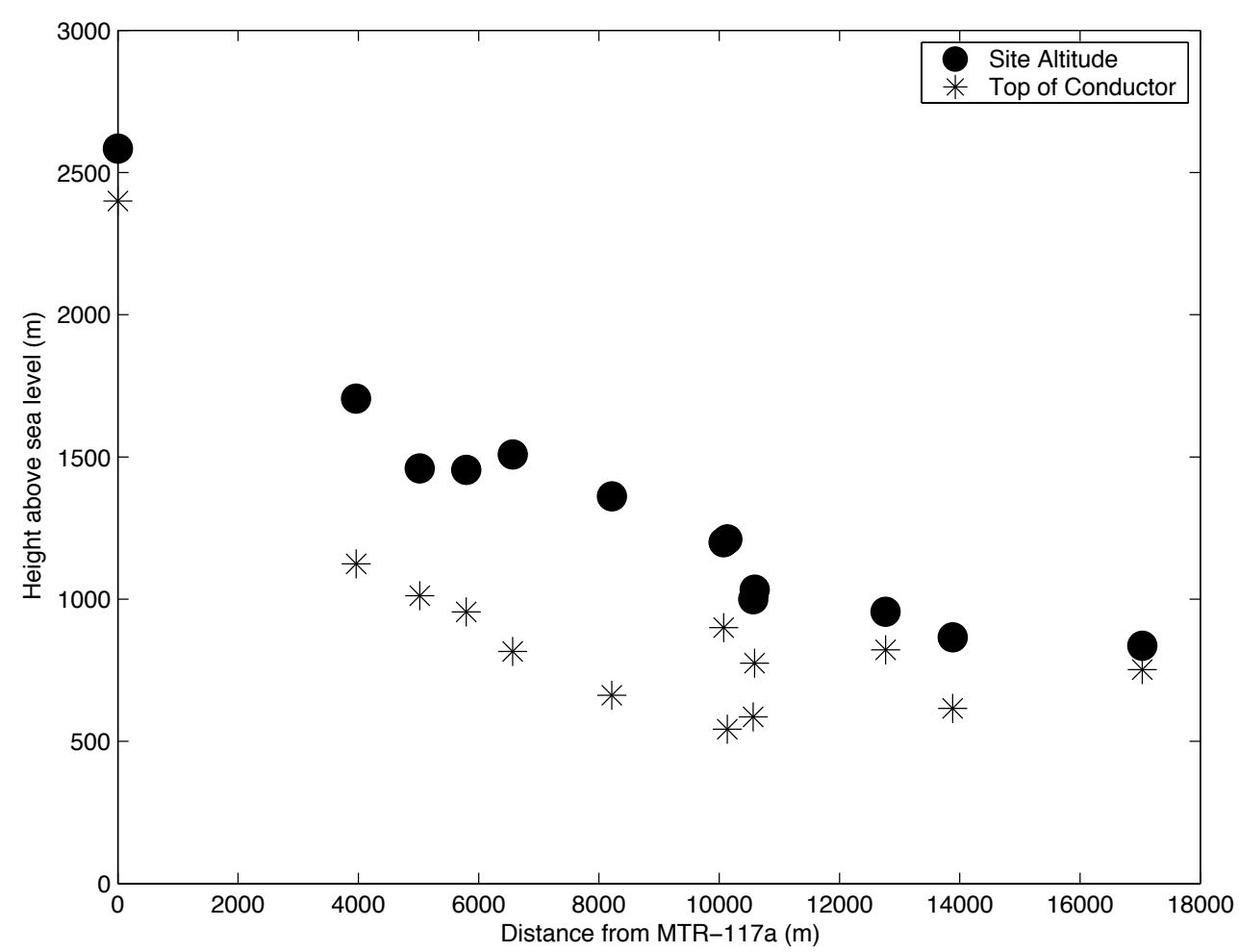

Figure 5.14 The height difference between the top of the shallow conductor and the site altitude.

conductive layer is an extension of the Tertiary sediment and not volcanic. East of the TVC there is no indication that this conductive layer exists.

The resistive layer beneath the conductor, which is seen at every site, is presumed to be the greywacke basement that is evident at the surface to the east (Grindly, 1960). Greywacke xenoliths have been found in Ruapehu lavas (Grange and Williamson, 1930, cited in Olson (1985)) indicating that a greywacke layer must be present beneath the TVC. This layer has a large range of resistivities $(100-2000 \Omega m)$ and thicknesses dependant on the presence of other layers.

The fourth layer that was present in a number of models, is a secondary conductor between 10 and $30 \mathrm{~km}$ deep. The resistivity values have a large range, due to nonuniqueness of the modelling, but this conductor is generally an order of magnitude lower in resistivity than the previous layer. The layer is most evident on the eastern flanks, but possibly also extends beneath the volcano as it was also modelled in site MTR-122a. Possible explanations of this layer are that it is an extension of the low velocity zone identified in the TVZ (Bannister et al., 2004; Ogawa et al., 1998), or that 


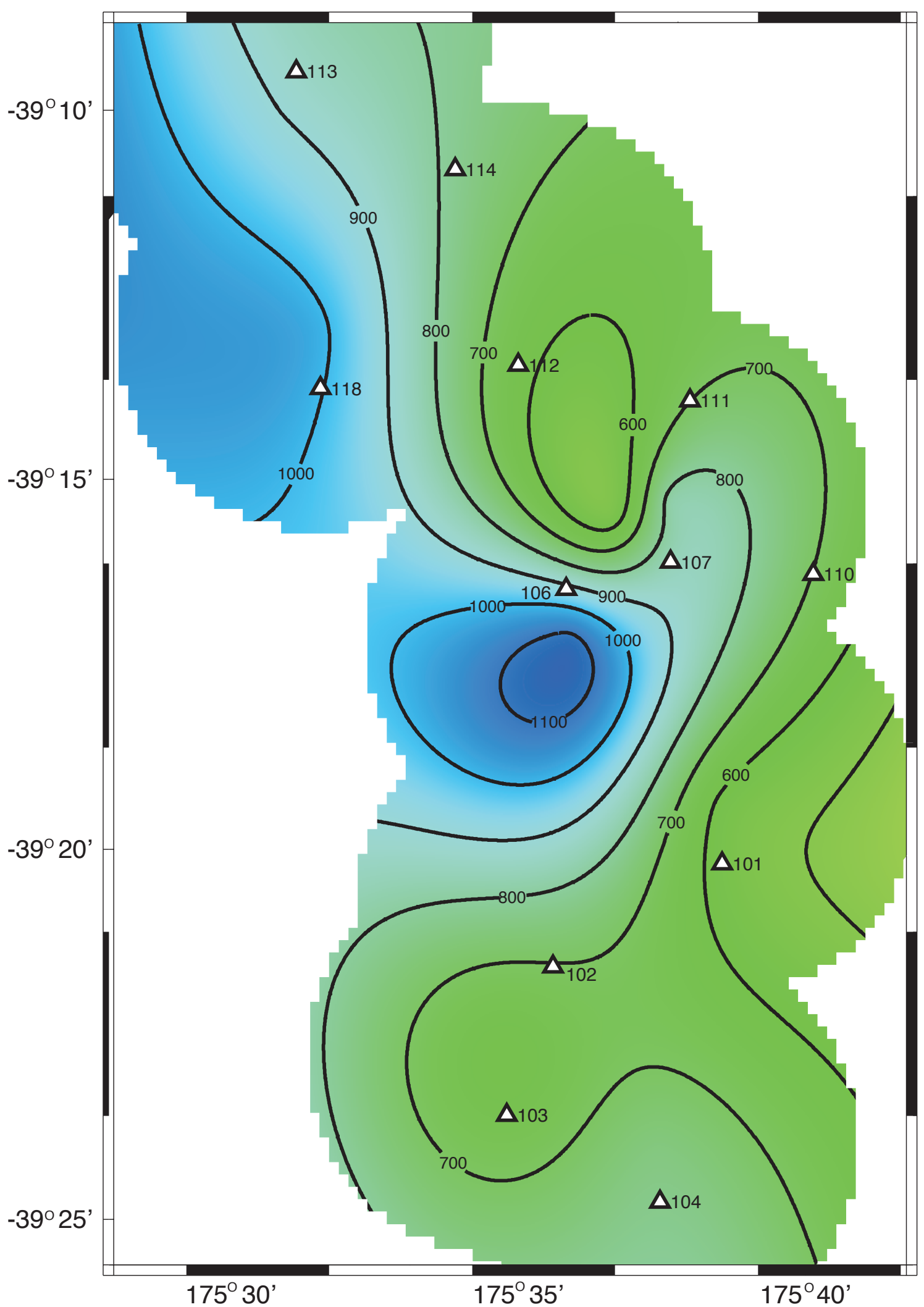

Figure 5.15 Contour map of the top surface of the shallow conductor identified in 1-D models. 
it contains the magmatic source supplying the volcano.

Below the secondary conductor the resistivity properties of the layer are similar to those recorded above the conductor in the third layer. Sites that do not show the secondary conductor often had an order of magnitude increase in resistivity at $80 \mathrm{~km}$ deep. This deep layer is probably due to large regional structures that are physically outside of the area of interest. At these depths the interpretation is based solely on the 1-D models, which are only indicative of the structure.

The models give a good fit with the data (see appendix $\mathrm{C}$ for graphs of the fit) and the comparability between near sites indicates that the derived structures are reasonable. However, 2-D models of the TVC boundaries will provide more constraints on the layers, which can then be tested as a 3-D forward model.

\section{$5.2 \quad 2-\mathrm{D}$ Modelling}

2-D models were created for the western and eastern boundaries of the TVC. Previous analysis of the phase tensor ellipses and accompanying parameters, ellipticity, $\beta$ and strike, all indicated the existence of 2-D structures in these locations.

The western boundary coincides with an extension of the Waimarino Fault (Hurst and McGinty, 1999; Lecointre et al., 1998) — a north/south striking normal fault down thrown between 200 and $60 m$ to the east (Pulford and Stern (2004) and Hurst and McGinty (1999) respectively). The most likely 2-D boundary in the eastern profile is the Desert Road Fault - a prominent north-northeast striking, west dipping, active normal fault (Villamor et al., 2004; Donoghue and Neall, 2001). This fault has a large surface escarpment and is up thrown to the east where basement greywacke outcrops are common.

The locations of the profiles were determined by plotting a line perpendicular to the strike direction that passes through the average latitude and longitude of the sites involved. Each site was then projected onto the profile line into the positions shown in figure 5.1. The data were then rotated into the strike direction for modelling. The 2-D inversions of the data used the "rapid relaxation inversion" method (Smith and 
Booker, 1991). This code is designed to find the minimum structure that fits the data.

All models start by assuming an $100 \Omega m$ half space. For each iteration the theoretical response from the model is calculated, compared to the data, then the model is altered until no further improvements to the misfit can be made. Each run included four types of inversions, in this case the program ran 20 non-robust, 10 robust, 20 redescending, and 20 smoothing inversions. These numbers represent the maximum number of each inversion type to be run unless the misfit failed to improve after a few attempts in which case the program automatically moved onto the next type of inversion. Both profiles were modelled in a joint inversion (TE and TM modelled simultaneously), and separate TE and TM mode inversions and with varying parameters (details follow for each profile).

The models were created assuming there was no topography. Following the inversion, the calculated depths were shifted upwards by the altitude of the site in an attempt to accommodate topographical variations in a method similar to that of Ogawa et al. (1998). From the phase tensor analysis it can be seen that the inductive effects of topography affected a significant amount of the data involved in these inversions. Frequencies that exhibited 3-D properties were therefore excluded from the inversion input file. After the inversion and altitude shift the resistivity values were contoured to create a smooth cross section of the resistivity profile in GMT (Wessel and Smith, 2001).

In addition to the profiles shown in figure 5.1, a number of other areas indicated 2 dimensionality within narrow frequency bands, outside of which the data were largely 3-D. If the common distortion assumptions are applied (where all higher frequency 3-D data are classified as resulting from a heterogeneity and therefore removed before inversion (Groom and Bailey, 1989)) these profiles could be inverted. In this case it is very unlikely that the physical structure causing this 2-D response is directly below the sites involved, thus inverting these profiles will have no value in determining the structure below Mt. Ruapehu.

Rough initial models of these profiles showed large lateral gradients, an artefact that Smith and Booker (1991) commented on in their introduction of the rapid relaxation inversion code. Positive delta functions (Parker, 1980) developed during the 
initial stages of the inversions, occurring at similar depths below different sites and can introduce a large horizontal gradient that remains during subsequent iterations (Smith and Booker, 1991). These models showed so little realistic structure that the profiles were not developed further.

Due to the $90^{\circ}$ ambiguity that is a property of strike direction determination, orthogonal profiles were also developed. The unrealistic nature of these orthogonal models, teamed with the large final misfits strongly suggest that the 2-D structures influencing the data have an approximately north/south strike in each case. The two profiles that cut the structure are referred to as profile A and profile B and are discussed separately below.

\subsubsection{Profile A}

Profile A intersects the western boundary of the TVC. Previous mapping of the faults within the area indicate that the Waimarino Fault terminates south of the profile (see figure 5.1), although from the topography it is reasonable to assume that a number of additional faults may have operated in the area at some time. Sites MTR-119a and MTR-120a are outside of the TVC and are situated on Tertiary sediment. The other sites are within the TVC on the ring plain of Mt. Ruapehu and are situated directly on volcanic debris. The final model obtained from the inversion process is presented here as figure 5.16.

From the dimensionality analysis, the structure in the area does not become 3-D until periods are longer than $10 \mathrm{~s}$. This frequency range corresponds to depths above 9 $\mathrm{km}$ when surface resistivity is taken to be $30 \Omega \mathrm{m}$. All data points that were determined to be 1-D were automatically included in the inversion file. 2-D data were included when the strike angle was consistent (taking the $90^{\circ}$ ambiguity in strike calculation into consideration) with the general pattern.

Each profile was modelled with varying parameters. The difference between models created from inverting the TE and TM modes separately were negligible. These separate mode inversions were also consistent with the joint inversion presented here. 


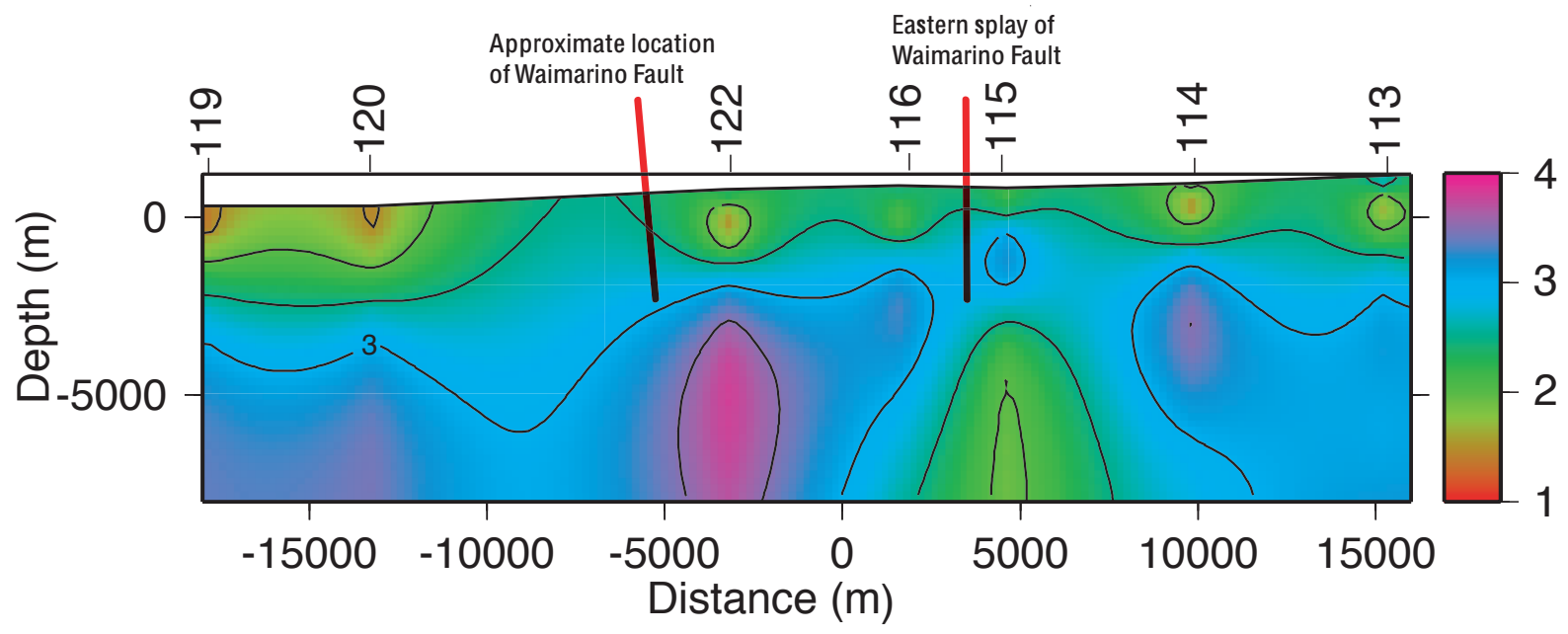

Figure 5.16 The 2-D model produced from Profile A

Furthermore, alternative modelling parameters had little effect on the structure presented here. The main parameters used to create this model are:

- The relative error floor for the apparent resistivity was $0.05 \%$ of the Log 10 of the magnitude.

- The relative error floor for the phase was $0.05 \%$ of the angle.

- No set distortion "matrix" or "parameters" were applied to any site.

- Automatic static shift was not allowed.

- Residuals were smoothed.

- The reduction in the error at any step had a maximum factor of 0.7 .

The first feature noticed (in figure 5.16) is that the surface conductor in the west is not continuous across the fault trace, although five of the seven sites contain a conductive near surface feature (below $100 \Omega m$ ). The remaining sites, MTR-115a and MTR-116a still contain a relatively low resistance in this upper section. In the west, beneath sites MTR-119a and MTR-120a, the conductor may be confidently identified as Tertiary sediment. If the conductor indicated by the 1-D modelling was an extension 
of this, it is reasonable to expect it to be continuous across the western TVC boundary. The lack of continuity on the other hand would support the hypothesis that the conductive layer on the east and north flanks of the volcano has a different cause.

The derived 2-D model does not show any direct evidence of the Waimarino Fault although a boundary of some nature does appear to exist between sites MTR-115a and MTR-116a. This may represent an extension of the eastward splay of the Waimarino Fault. If the fault does cross the profile to the west of site MTR-122a then either the model resolution or resistivity contrast are insufficient for the fault to be identifiable within the current data set. It is possible that the conductor present to the east is the extension of the Tertiary sediments and that the eastern fault splay has disturbed the sediments near sites MTR-115a and MTR-116a causing the discontinuity seen in the model.

After the inversion was completed the derived structure was then used in a forward model to show how well this structure reproduced the original data. The comparison graphs for all sites involved in the 2-D modelling of each profile are presented in appendix C, and have a good fit.

\subsubsection{Profile B}

Profile B crosses the eastern margin of the TVC. Here, site MTR-105a is outside the TVC in an area where basement outcrops of greywacke are common. The other five sites involved in this inversion are on the eastern flank of the volcano. This profile was designed to show the extent of the conductor below the volcano and to determine if a magmatic system could be seen. The final model obtained from the inversion is presented as figure 5.17.

Again the varying parameters and different modes of inversion had little effect on the structure presented in the final joint inversion model. The final options used to model Profile B are the same as those presented for Profile A. The restricted range of frequencies used in the inversion prevent the deep structure from being resolved. In this case data at greater than $2 s$ period were determined to be 3 -D and therefore excluded from the inversion file. This frequency range corresponds to depths above 7 


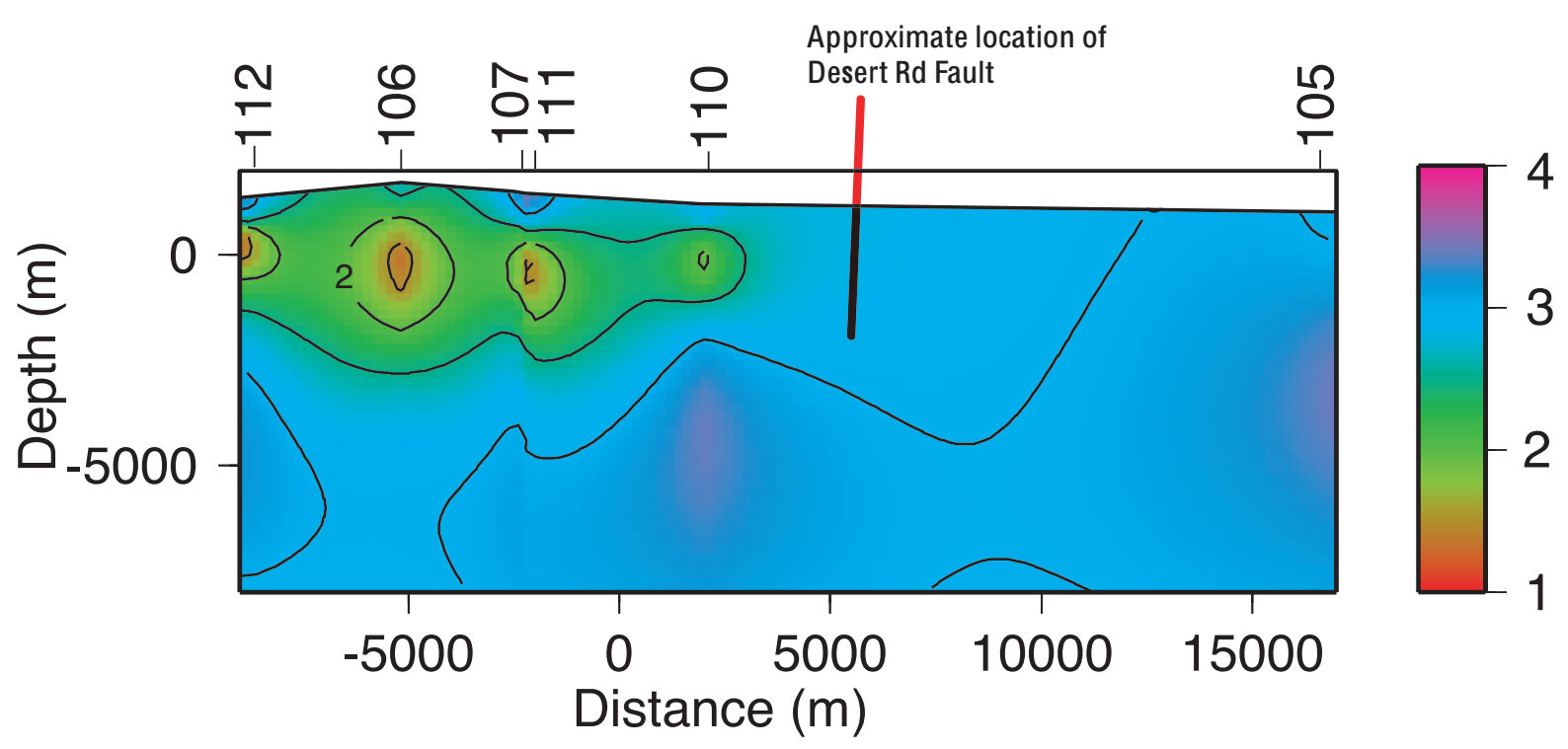

Figure 5.17 The 2-D model produced from Profile $B$

$\mathrm{km}$ when the surface resistivity is taken to be $100 \Omega \mathrm{m}$.

The derived 2-D model clearly shows a conductor (resistivity $10-30 \Omega m$ ) to be present beneath the eastern slopes of the volcano. This conductor does not extend as far as site MTR-105a. The thickness of the conductor increases to the west of the profile supporting the conjecture that it volcanic in origin. It would be expected that material of volcanic origin would be thicker beneath the centre of the volcano. The lens shape of the conductor in this model may be due to site MTR-112a being further from the centre of the volcano than site MTR-106a despite being further west in the profile.

Unfortunately this profile lacks sites between sites MTR-110a and MTR-105a that could determine where the conductor ends. The presence of the National Power Grid in a north/south direction inhibits the collection of natural electric field variations between these sites.

Apart from the conductor present beneath the volcano, the conductivity structure of the volcano is very uniform at an average resistivity of $1000 \Omega m$, as would be expected of continental crust. The limited depth resolution prevented any modelling of the second conductor identified in 1-D models. 


\subsection{Comparison of 1-D to the 2-D Models}

For each model created the fit between the input data and the model response were graphed, these graphs are presented in appendix C. Each graph shows that the data modelled produced a reasonable fit when compared to the input data. However as the 1-D models were created using the determinant of the impedance tensor and the 2-D models were created using limited rotated 2-D data, there are differences between the models. Some of these differences are likely to be due to the non-uniqueness or different emphasis of the modelling procedures. The 1-D modelling program used a small number of distinct layers to fit the data, while the 2-D program used many layers to create a smooth gradually changing structure.

Despite the difference between the models, the features identified in the 2-D models can be compared to the features identified in the 1-D models of the sites involved. This may identify which structural features are required to obtain a good fit to the observed data.

- Profile A: There is a good comparison between the layers identified in the 1 and 2-D models. Although the shallow conductive layer identified below each site in both models is not continuous in the $2-\mathrm{D}$ model. The resistivity values given for each of the layers are comparable between the different models. However the 1-D models of sites MTR-115a and MTR-122a do not show any evidence of the vertical structures that are evident in the $2-\mathrm{D}$ profile.

- Profile B: The 1-D models have identified very similar structures to the 2-D model. This is particularly evident below site MTR-105a. However, the resistivity of the near surface conductor below the eastern flank of the volcano is higher in the 2-D model. As the resistivity values are higher in the 2-D model, the thicknesses of the surface layers increases although the parameters defining the layers vary, the existence and conductance of the layers are consistent.

From the comparison between the 1 and 2-D models, and the fit of the data, it can be assumed that these models are of good quality. As the shallow features were 
identified in both sets of models it is probable that these features do exist. However the difference between models below site MTR-115a on profile A suggests that the structure in this area is still largely undefined.

\subsection{Testing the Derived Structure in 3-D}

A number of features were identified during 1 and 2-D inversions of the data. These features were combined and simplified to develop a simple 3-D structure of the volcano. This simplistic structure was used to create a 3-D mesh that was then forward modelled in WinGLink (GEO, 2004) to test how well theoretical data from this simple 3-D structure matched the data observed in the field.

A 3-D mesh was created by defining an internal grid that encompasses all of the site locations. Beyond this grid extra lateral mesh was included as the program deemed necessary to eliminate edge effects. WinGLink restricts the number of grid cubes that can be included in the forward modelling step due to computer limitations. Consequently for the area of the internal grid the model created had a lateral grid line spacing of $1000 \mathrm{~m}$ in both the north/south and east/west directions. For the above and near surface ranges the vertical grid lines had a spacing of $100 \mathrm{~m}$. The lowest site altitude is $300 \mathrm{~m}$ above sea level; therefore below this altitude it was only necessary to include a grid line where a boundary was needed to make the model agree with the identified structure.

The large cubic mesh encompassed all the cubes to be modelled. As it is possible to assign an 'air' resistivity value to any cube, the shape of the topography could be included using 'air' cubes to define the surface, although the large grid spacing restricted this to the large scale topography. Müller and Haak (2004) identified that if the slope of the volcano is represented as a series of steps as in figure 5.18(a) then the edge effects of the cubes may greatly decrease the model fit, but if the slope was modelled as in figure 5.18(b) a better overall fit between the modelled and observed data was obtained. To the extent that it was possible, this reduction of edge effects was included in this mesh. Where there were large altitude differences between sites that were laterally close it was not always possible to apply this adaptation. 


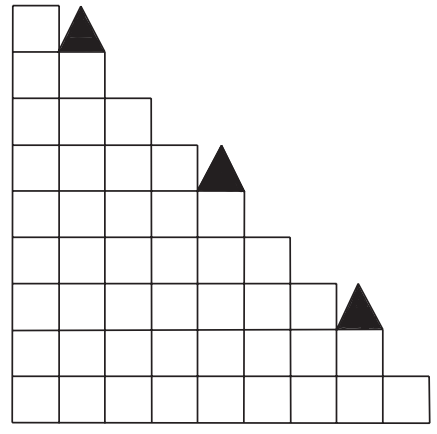

(a)

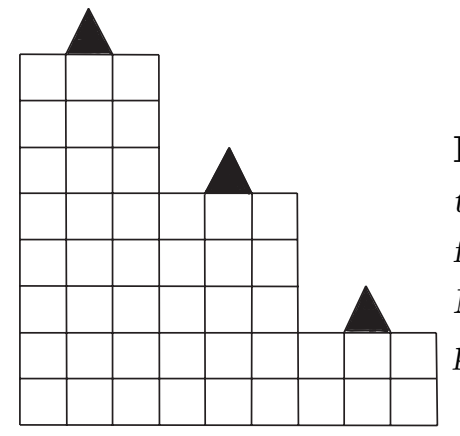

(b)

Figure 5.18 Griding of steep topography to obtain the best fit to actual data. Taken from Müller and Haak (2004) and applied in 3-D forward model

The locations of the sites occupied during the survey were superimposed onto the mesh. The resistivity structure determined during the inversions was entered into the corresponding grid cubes. Resistivities were then interpolated between cubes until the 3-D mesh was full and the structure matched that outlined above. The following figures show cross sections through the mesh in both the east/west (figure 5.19) and horizontal (figure 5.20) directions.

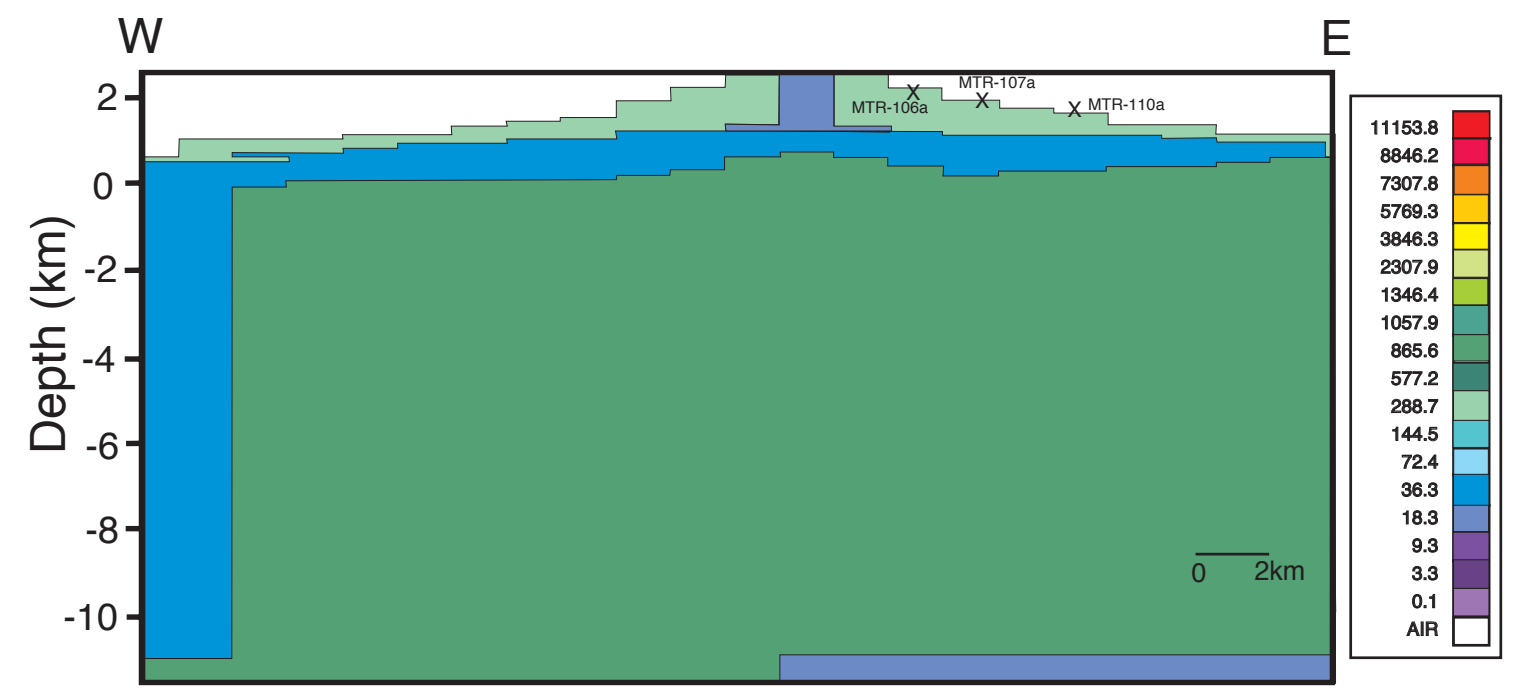

Figure 5.19 An east/west cross section from the 3-D mesh that was modelled to test the validity of the structure defined by earlier inversions

In the vertical cross section (figure 5.19) the main features of the previously identified structure can be seen. These include,

- A resistive surface layer on the flanks of the volcano.

- A conductive vent in the center of the volcano. 
- A shallow conductor beneath the volcano that extends to south and west of the TVC.

- This conductor is extended to $\approx 10 \mathrm{~km}$ deep west of the TVC.

- A second conductor below the eastern flank of the volcano (just visible in the bottom right of figure 5.19), this extends down to $30 \mathrm{~km}$.

- A resistive layer that is on the surface to the east, as well as between and below the conductors in all other locations.

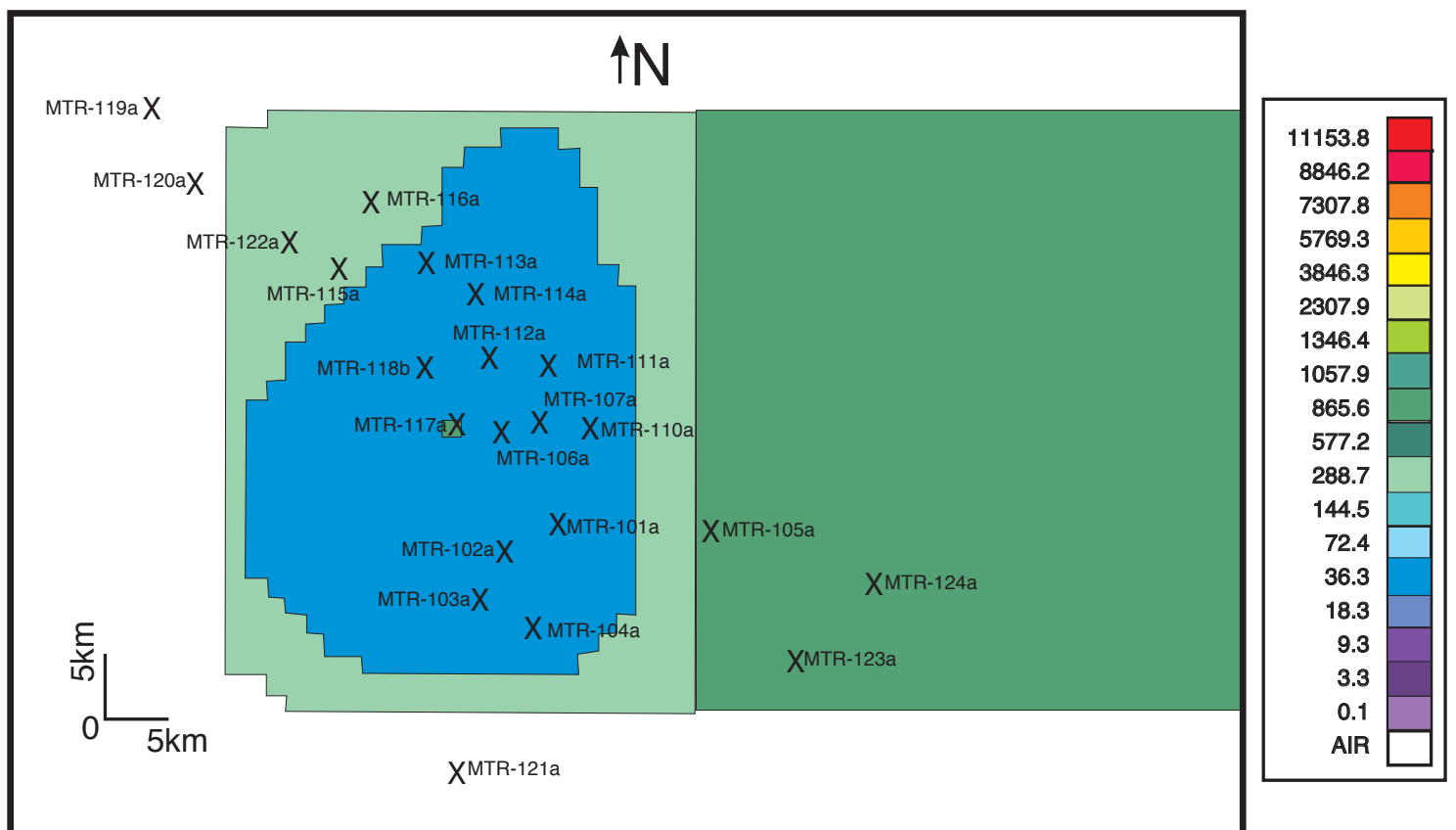

Figure 5.20 A horizontal cross section from the 3-D mesh that was modelled to test the validity of the structure defined by earlier inversions

In the horizontal cross section (figure 5.20) the main features of the previously identified structure can again be seen. These include,

- A central conductive vent below site MTR-117a.

- This is surrounded by the shallow conductor, the extent of this layer varying with the altitude of each slice.

- This shallow conductor is surrounded by the resistive surface layer (near low altitude sites in this slice of the mesh). 
- To the east of the TVC is the outcrop of greywacke basement.

This mesh was then forward modelled so that impedance tensor elements were calculated at each of four frequencies for each decade, the longest period was set to 1000 $s$. In order to compare these results to the data collected, the program was instructed to create a response file at each of the locations where a real site was located. These files could then be plotted in the same way that the original files were plotted using the Matlab script. The apparent resistivity and phase curves where plotted on top of the original apparent resistivity and phase curves for each site. For a complete set of these plots refer to appendix $\mathrm{C}$ although an example is presented below.
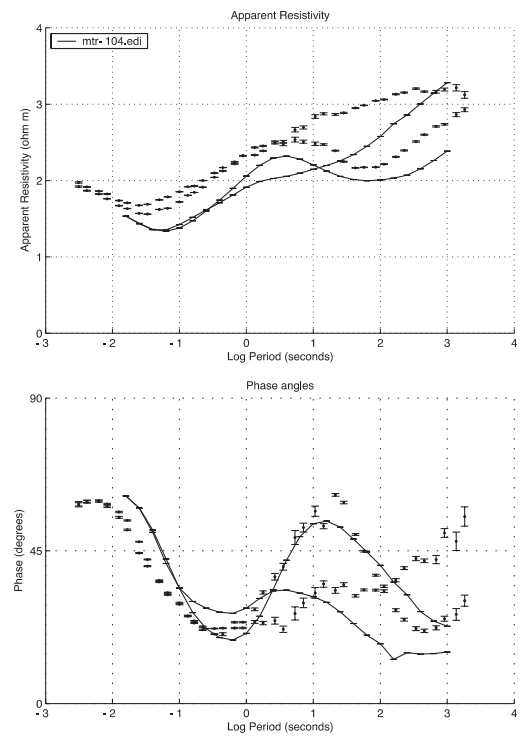
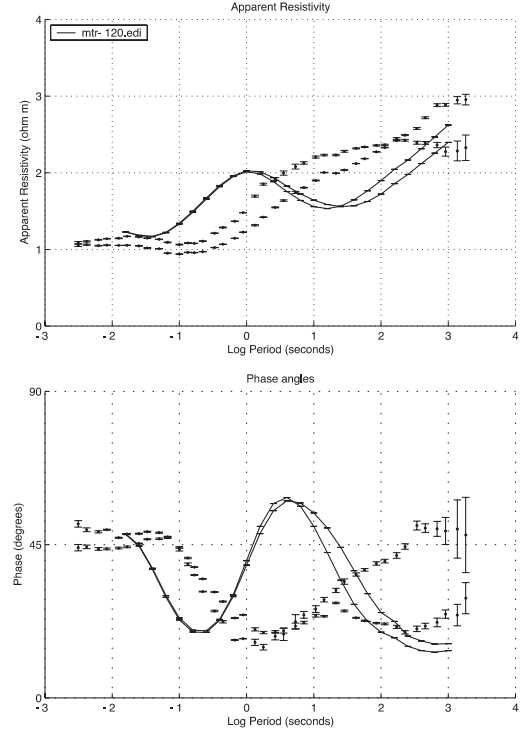

Figure $\quad 5.21 \quad T w o$ examples of apparent resistivity and phase curves determined from the results of the 3-D forward model plotted on top of the collected data from their respective sites.

From the apparent resistivity and phase curve comparison it can be seen that the degree to which the model fits the data varies significantly from site to site. This is unsurprising given the coarseness of the model. What is verified by the test is that many of the main features of the volcano's structure have been identified.

The poorest fit is for the long period data. The cause of this mismatch is that the MT data are effected by lateral variations in the electrical structure as well as vertical variations. In the mesh only close variations in lateral structure could be included. It is probable that long period data collected on Mt. Ruapehu are influenced by features such as the coast line or subduction zone to the east of the area, which were not be 
included in the model.

The higher frequency data are a better fit, although the model and actual resistivity values do not show a clear match. A vertical shift of most curves greatly reduced this misfit. This is the effect of simple static shift. The shapes of the model curves at high frequencies are often similar to the observed data indicating that the main features of the structure have been included in the model.

There are also variations in the quality of the fit between sites. Figure 5.21 is an example of a site with a reasonable fit between modelled and observed data (site MTR104a), and a site with a poor fit (site MTR-120a). In general the sites within the TVC have a closer correlation to observed data.

Many modelled curves within the TVC have a good shape match at the high and mid frequencies. A vertical shift would be appropriate in many cases to obtain a better fit. In appendix $\mathrm{C}$ the modelled data have been manually shifted to account for static shift. The first minima in some of the apparent resistivity curves occur at a slightly longer period than it does in the observed data. This horizontal shift is present at sites MTR-104a (figure 5.21) and MTR-113a and is likely to be due to the thickness of the upper layers. The misfit probably originates from altering the depth of the first resistor/conductor boundary to provide continuity between adjacent sites.

Outside the TVC the response from the 3-D model have less similarity to the observed data. To the east where a narrow range of apparent resistivity values were observed, the models match the average resistivity value but not the minima and maxima observed. There is also a large split between the modelled curves starting at a period of $0.1 \mathrm{~s}$, which was not seen in the observed data.

To the west of the TVC and the sites included in the 2-D inversion of profile A there are significant differences between the modelled data and the observed data. Sites MTR-113a, MTR-114a, and MTR-122a all show a reasonable fit, but the other four sites do not. The model response at sites MTR-115a and MTR-116a are similar to those of site MTR-113a and MTR-114a, but the observed data are quite different. Sites MTR-115a and MTR-116a do not show evidence of the conductor and the apparent resistivity shows a steady increase with period, which is matched by one mode of 
the model response but not the other. West of the Waimarino Fault the apparent resistivities for the sites MTR-119a (figure 5.21) and MTR-120a increase at a higher frequency than the observed data do. The modelled data from these sites also show an influence from the second conductor placed under the east of the volcano, the observed data did not show any evidence that it was being effected by this feature. This combined with the split in sites east of the TVC, may be an indication that the deeper conductor has been overemphasised in the 3-D model.

The phase data for all sites has a poorer fit than the apparent resistivity data. As minima and maxima are identified at shorter periods in the phase than the apparent resistivity the loss of fit at long periods in the apparent resistivity data is reflected at shorter periods in the phase data. There is also a poor fit to data within the dead band where the phase was often poorly defined.

An alternative way of testing how well the modelled data fits the observed data is to compare the phase tensor ellipses. Figure 5.22 shows the comparison of the theoretical phase tensor ellipses (black) over laying the observed phase tensor ellipses (red) at a range of comparable frequencies. The phase tensor ellipses confirm the observation that the structure modelled fails to match the observed data to the west. However in general there is a good fit between the modelled and observed ellipses at high frequencies. In the dead band the quality of the fit begins to decrease, and at times the ellipses are even perpendicular to each other. The long period ellipses again appear very similar in orientation although the ellipticity of the modelled ellipses are larger than the observed ellipses.

From the comparison of the observed data to the model responses it can be seen that most of the shallow features of the volcano's structure have been identified. However the poor fit of the western data suggests that the simplified structure used for the 3-D test is inappropriate for modelling this data. Due to the limited lateral extent of the model many of the long period features were not reproduced.

The results of this 3-D modelling have shown that the main shallow features of the volcano were identified in the 1 and 2-D inversions presented earlier. The averaging of the properties of the layers to create a simple model may account for the static shift 

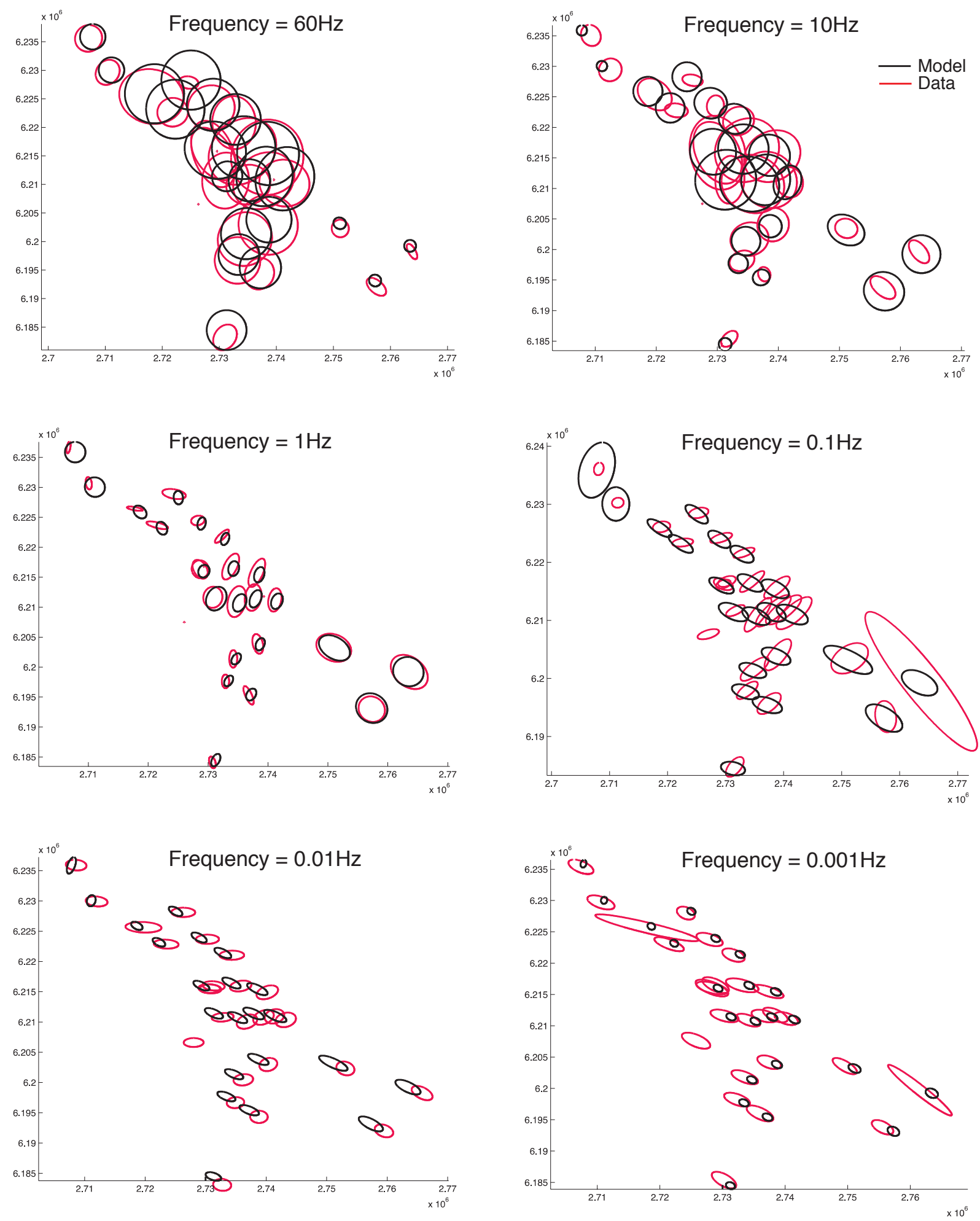

Figure 5.22 The phase tensor ellipses derived from the results of the original 3-D forward model compared to the original phase tensor ellipse maps at similar frequencies. 
that was present in the model fits. The higher quality of the model fit beneath the volcano indicates that the major features of the volcano have been identified. 
CHAPTER 6

\section{DISCUSSION}

This chapter compares the results of this study to studies previously conducted with the aim of clarifying the results of the modelling and trying to provide answers to the questions set out at the start of this thesis. Summing up with the final conclusions from this work and identifying ideas for further work.

\subsection{The Existence of Magma}

Primarily this study aimed to determine if a coherent body of magma existed beneath the volcano. From the 1-D models calculated it can be seen that there are no shallow conductors, other than those clearly identified with wet/altered volcanic debris and Tertiary sediments, beneath any of the sites occupied in this survey. However, below the eastern flank of the volcano a second deeper conductor is identified at a depth of $\approx 10 \mathrm{~km}$. Although the resistivity value of this conductor is higher than values recorded for molten magma itself, it is possible that this layer is related to the heat source for the volcano.

Hermance and Colp (1982) identified magma through electrical techniques and stated that the resistivity of magma is between $0.2-2 \Omega m$. This is one to two orders of magnitude lower than the most conductive coherent body identified beneath Mt. Ruapehu.

Hydrothermal activity is visible within the hot acidic Crater Lake and easily accounts for the low resistivity recorded at the summit. Hurst et al. (1991) showed using energy and mass balance equations that Crater Lake and the vent system beneath act 
as a heat pipe with a deep magmatic heat source keeping the vent as a hot fluid body. This heat pipe system does require the existence of a magmatic heat source within the volcano, but without constraints on the depth. However, there is a possibility of a shallow magma chamber existing beneath the southwestern flank of the volcano as the only data from this region are the long period data collected by Hanekop (2002), which simple skin depth calculation indicated that the highest frequency measured in that survey correlated to a depth of $\approx 16 \mathrm{~km}$.

The other possibility is that instead of a coherent magmatic heat source, this volcano may be feed by a number of small disconnected vents surrounded by rock material or cooled magma that never made it to the surface. If this is the case then the apparent resistivity value associated with the system could be much higher, as it was in Long Valley, California where Park and Torres-Verdin (1988) attributed resistivity values higher than $30 \Omega m$ to the existence of magma.

Therefore the second conductor identified at a depth of $\approx 10 \mathrm{~km}$ may be a possible heat source driving the volcano and no coherent magma chamber exists elsewhere. Using properties of bulk resistivity, and the assumption that this is a mixture of pure melt and basement greywacke the Hashin-Shtrikman limits (presented and discussed as equations 2.1 and 2.2 in chapter 2 ) indicate that this conductor would be approximately $13 \%$ pure melt. For this calculation the bulk resistivity was assigned a value of $20 \Omega m$, the melt assigned a value of $1 \Omega m$ and the greywacke a value of $400 \Omega m$.

\subsection{The Structure of the Volcano}

Much data have been collected over the years concerning the surface geology of the volcano and surrounding area. One aim of this survey was to add details to the poorly defined deeper structure.

1-D modelling of the data indicated that less than five layers were needed to model most sites, however these layers vary somewhat between sites. A resistive surface layer was modelled for each of the sites within the TVC. This layer has a resistivity of $100-400 \Omega m$ and is up to $500 m$ thick. Taking lithology from the geological map 
(Hay, 1967; Grindly, 1960), within the TVC this layer can be identified with Quaternary volcanics. Higher resistivity values for this layer were modelled at the sites near the summit, consistent with the presence of more resistive solid lava flows near the surface.

Below this layer and extending beyond the TVC in the south and west is a conductive layer. Outside the TVC this layer outcrops at the surface and has been mapped as Tertiary sediment (Hay, 1967; Grindly, 1960). Although a doming effect was noted around the volcano suggesting a volcanic association, the resistivity of the entire layer was modelled between 10 and $30 \Omega \mathrm{m}$. A value consistent with both the resistivity values recorded for altered volcanic debris (Bibby et al., 1998) and Tertiary sediments (Ingham et al., 2001) in other parts of New Zealand.

A common resistivity feature of other volcanoes modelled using the MT method is the presence of a conductive layer (Manzella et al., 2004; Matsushima et al., 2001; Ogawa et al., 1998) with comparable resistivities to the above conductor. In these cases the cause of the conductor was interpreted to be a presence of wet fractured volcanic debris, possibly with associated low temperature alteration within pyroclastic material. As this altered volcanic debris has a similar resistivity value to Tertiary sediment it is not possible to distinguish between them using electrical methods.

It is most probable therefore that this layer represents both lithologies. Tertiary sediment does exists within the TVC, and is presumed to extend beneath the volcano. A low velocity layer has been identified in seismic studies (refer to figure 2.5) and interpreted to be an extension of the Tertiary sediment (Sissons and Dibble, 1981). This Tertiary layer can be seen in road cuttings in the western TVC and is known to outcrop at $800 \mathrm{~m}$ (asl) beneath Mount Hauhungatahi, which has acted as a volcanic cap since it erupted (Pulford and Stern, 2004).

Pulford and Stern (2004) calculated that the Tertiary sediment would have eroded another $500 \mathrm{~m}$ since the capping of Mount Hauhungatahi, although once Mt. Ruapehu produced its own lavas it would have prevented further erosion of underlying sediment. Mt. Ruapehu's first lavas (the Te Herenga Formation, approximately 130 thousand years ago) are much younger than much of the exhumation and uplift and therefore layers above the Tertiary sediments and possibly the top of the Tertiary would have 
already been eroded to the extent that volcanic material was erupted directly onto an erosional Tertiary surface. Using exhumation rates and the age of the oldest lavas identified, the top of the Tertiary sediment below the volcano should be approximately $300 m$ (asl). Both the 1 and 2-D models have the conductor placed higher than this.

The 2-D profile crossing the western boundary of the TVC shows that there is no resistivity contrast associated with the main trace of the Waimarino Fault. However, there is an area of high conductivity that coincides with an extension of the eastern splay of the fault. The conductor is not continuous across this boundary tending to suggest that the conductor beneath the TVC is not a continuation of the Tertiary sediment. When this structure was tested in the 3-D forward model a match was not achieved. Aspects of the structures just east of the fault are still undefined, and additional measurement sites are needed in order to understand what is happening in this location.

The conclusion therefore is that the near surface conductor is a combination of fractured or altered volcanic debris overlying Tertiary sediment. The boundary between these layers is approximately $300 \mathrm{~m}$ (asl) but not definable through electrical methods as the conductivity of the two materials are too similar to distinguish between them.

Below this shallow conductor there is a resistive layer that is consistent with greywacke basement. This layer has a resistivity of 200-1000 $\Omega m$, and extends beneath all the sites containing the conductor in addition to being the surface layer (Hay, 1967; Grindly, 1960) below sites to the east of the TVC. The presence of greywacke xenoliths in Ruapehu lavas (Grange and Williamson, 1930, cited in Olson (1985)) indicate that greywacke must also be present beneath the volcano. Therefore it is probable that this entire layer is greywacke basement.

Below this resistive layer at sites on the eastern flank is a second conductor . This second conductor is between 10 and $30 \mathrm{~km}$ deep and represents a drop in resistivity of half an order of magnitude (averaging $20 \Omega m$ ). As mentioned above in the discussion on the existence of magma, this layer may contain $\approx 13 \%$ melt and is probably the driving heat force of the volcano. However 3-D testing of this layer suggests that it was over emphasized in the 3-D mesh and is probably a lot thinner than the $20 \mathrm{~km}$ 
modelled. As this layer was identified through the 1-D modelling the constraint on the properties were ill defined.

There is also a possibility that this conductive zone is a continuation of the low seismic velocity zone identified beneath the TVZ at $15 \mathrm{~km}$ deep. If this is the case then the same narrow zone of melt may be the source of all the volcanic and geothermal activity within the TVZ.

The data collected by Hanekop (2002) and in this current survey are consistent and indicate that over the last two years there have not been any detectable change in the deep structure of the volcano.

\subsection{The Phase Tensor Ellipse}

An additional aim of this study was to apply the phase tensor ellipse method to provide insights into structure and to remove any distortions from the data set without applying the standard 2-D assumption inherent in other techniques. This distortion removal method (Bibby et al., In Press) proved easy to apply as the dimensionality analysis showed 1-D sections of the curves. A simple alteration to the Matlab script provided by Dr. Bibby (2004, pers.comm.) allowed these sections of the curve to be used to calculate the distortion tensor. This tensor could then be applied to the entire frequency range to remove distortion.

The long period data collected by Hanekop in 2002 closely resembles those collected in this survey at sites MTR-107a and MTR-118a, which are located very close to sites TUK and WHA respectively. Theoretically the long period data should not contain significant distortion. Hanekop did not apply any distortion removal technique to his data and the comparison indicates that the phase tensor distortion removal process did not hinder the long period data.

The phase tensor ellipses provided great insight into features influencing the EM induction at different frequencies. The high frequency data were clearly influenced by the local topography around each site. When the phase tensor ellipses were plotted onto a topographical map the major axis of the ellipses lines up with significant topography. 
At longer periods a 2-D boundary was seen to the northwest near the Waimarino Fault. This provided quick identification of an area for a possible 2-D modelling profile. 2-dimensionality of the data from this area was confirmed once the phase tensor invariant parameters were calculated. Again this was the case for the eastern boundary near the Desert Road Fault.

The most impressive feature of the phase tensor ellipse maps was the uniformity of the major axes at long periods. This indicated that all the sites were responding to the same deep conductivity structure. Regional studies of the area indicated a northnortheast regional strike. Below these frequencies the major axes rotated uniformly to the next major conductivity boundary. Ingham et al. (2001) showed that high conductivity was associated with the east coast and possibly the subduction zone beneath it. Either of these causes are a possible explanation for the alignment of the very long period data.

This phase tensor method represents a new method in distortion removal and in this case has proved very effective. As Mt. Ruapehu is a 3-D feature applying the standard 2-D assumption inherent in previous methods was inappropriate in this situation. Additionally the analysis indicated that the effects of topography cause an inductive distortion down to $1 \mathrm{~s}$. This would need to be taken into consideration in any further 3-D modelling of the area.

This method therefore provides quick insights into the structures influencing EM induction, thus identifying the modelling types appropriate as well as providing a simple distortion removal process that does not require any assumptions on the dimensionality of the data. From this study there is no reason to doubt the validity or usefulness of this method.

\subsection{Conclusions}

To conclude, it is unlikely that a shallow magma chamber exists directly beneath the volcano. However the heat source for the volcano is likely to be associated with the secondary conductor identified at $\approx 10 \mathrm{~km}$ deep; feeding the volcano through a number 
of small disconnected dykes that are not resolvable through MT measurements. Under the assumptions made above it is possible that this layer contains $\approx 13 \%$ melt.

The electrical structure of the Mt. Ruapehu area consists of:

- A layer of volcanic debris and lava flows extending across the entire TVC. This layer has a resistivity of $\approx 100 \Omega m$ and extends to approximately $800 \mathrm{~m}$ (asl) with the thickness varying with distance from the vent.

- Between $\approx 300$ and $800 \mathrm{~m}(\mathrm{asl})$ is a conductive layer of wet fractured or altered volcanic debris. This layer has a conductivity of $10-30 \Omega m$ and is electrically indistinguishable from the next layer.

- The bottom section of this shallow conductor is Tertiary sediment and extends beyond the TVC to the south and west.

- Below the Tertiary sediment and outcropping on the eastern plateau is greywacke basement. The average resistivity value of this greywacke is $700 \Omega m$ extending to $80 \mathrm{~km}$ deep in the west and is interrupted at $10 \mathrm{~km}$ depth in the east.

- On the eastern flank of the volcano is a second conductive layer around $10 \mathrm{~km}$ deep, with an average resistivity of $20 \Omega \mathrm{m}$. This layer is the most probable heat source driving the volcano and possibly contains $13 \%$ melt.

- Below this second conductor, the sites consistently show a layer down to at least $80 \mathrm{~km}$ which has a resistivity value indistinguishable from the greywacke above.

The phase tensor analysis also showed that topography has a significant effect on high frequency data and needs to be considered. Additionally, with large structures such as Mt. Ruapehu, this inductive effect distorts all data with periods below $1 \mathrm{~s}$. Galvanic distortion removal does not attempt to remove this effect and therefore it needs to be included in modelling to retrieve a realistic model.

This method quickly and easily identified where data were reacting to a local 2-D structure. Both the Waimarino and Desert Road Faults distinctly effected the direction of the major axes in nearby phase tensor ellipses. This showed where it was appropriate 
to apply a 2-D inversion and indicated the frequencies that should be included in these inversions.

In the long period data the major axes of the phase tensor ellipses conformed to the direction of the regional strike determined for the TVZ and New Zealand as a whole. Furthermore as the period continued to increase all the ellipses rotated uniformly so that the major axes pointed to a southeast conductivity boundary.

\subsection{Further Work}

Higher resolution of the structure around the volcano could be obtained if appropriate 3-D modelling was to take place. This modelling would require the application of an inversion technique that takes into account the induction effects of topography. More MT sites are planned for Mt. Ruapehu, and these sites are to be located so as to make 3-D modelling appropriate. Additionally it would be interesting to collect more data around the edges of the TVC so that more detailed 2-D modelling could be completed on the boundary faults, particularly the northern end of the Waimarino Fault. 


\section{REFERENCES}

Ádám, A., Szarka, L., Verö, J., Wallner, Á. and Gutdeutsch, R. (1986), Magnetotellurics (MT) in mountains - noise, topographic and crustal inhomogeneity effects, Physics of the Earth and Planetary Interiors 42: pp165-177.

Alder, G. (1983), Seismic and gravity studies of Tokora gravity anomaly, North Island, New Zealand, Master's thesis, Victoria University of Wellington.

Bahr, K. (1991), Geological noise in magnetotelluric data: a classification of distortion types, Physics of the Earth and Planetary Interiors 66: pp24-38.

Bannister, S., Bryan, C. and Bibby, H. (2004), Shear wave velocity variation across the Taupo Volcanic Zone, New Zealand, from reciever function inversion, Geophysics Journal International 159.

Berdichevsky, M. and Dmitriev, V. (1976), Basic principples of interpretation of magnetotelluric sounding curves, KAPG Geophysical Monograph, Akadémiai Kiadó pp. pp165-221. Cited in Jones (1988).

Bibby, H., Caldwell, T. and Brown, C. (In Press), A coordinate invariant approach to distortion effects in magnetotelluric data, Geophysics Journal International .

Bibby, H., Caldwell, T. and Risk, G. (1998), Electrical resistivity image of the upper crust within the Taupo Volcanic Zone, New Zealand, Journal of Geophysical Reasearch 103: pp9665-9680.

Bibby, H. M. (1986), Analysis of multiple-source dipole-quadrapole resistivity surveys using the apparent resistivity tensor, Geophysics 51(4): pp972-983.

Bibby, H. M., Caldwell, T., Davey, F. and Webb, T. (1995), Geophysical evidence on the structure of the Taupo Volcanic Zone and its hydrothermal circulation, Journal of Volcanology anf Geothermal Research 58: pp29-58.

Bobachev, A. A. (1990), IPI2Win, Geoscan -M Ltd, Moscow, Russia.

Bostick, F. (1977), A simple almost exact method of MT analysis, Proceedings: Workshop on electrical methods in geothermal exploration 174-184, U.S. Geological Survey, Contract 14-08-001-6-359. Cited in Jones (1988).

Caldwell, T., Bibby, H. M. and Brown, C. (2004), The magnetotelluric phase tensor, Geophyscis Journal International 157: pp1-13. 
Calhaem, I. (1973), Heat flow measurements under some lakes in North Island, New Zealand, PhD thesis, Victoria University of Wellington, New Zealand.

Cole, J. (1978), Andesites of Tongariro Volcanic Centre, North Island, New Zealand, Journal of volcanology and Geothermal Research 3: pp121-153.

Cole, J. (1990), Structural control and origin of volcanism in the Taupo Volcanic Zone, New Zealand, Bulletin of Volcanology 52: pp445-459. Cited in Wahyudin (1993).

Constable, S., Parker, R. and Constable, C. (1987), Occam's inversion: a practical algorithm for generating smooth models from electromagnetic sounding data, Geophysics 52(3): pp289-300.

Darby, D., Hodgkinson, K. and Blick, G. (2000), Geodetic measurement of deformation in the Taupo Volcanic Zone, New Zealand: the north Taupo network revisited, New Zealand Journal of Geology and Geophysics 43: pp157-170.

Dibble, R. (1972), Seismic and related phenomena at active volcanoes in New Zealand, Hawaii and Italy, PhD thesis, Victoria University of Wellington, New Zealand.

Donoghue, S. and Neall, V. (2001), Late Quaternary constructional history of the southeastern Ruapehu ring plain, New Zealand, New Zealand Journal of Geology and Geophysics 44: pp439-466.

Ewart, A., Brothers, R. and Mateen, A. (1977), An outline of the geology and geochemistry, and possible petrogenetic evolution of the volcanic rocks of the TongaKermadec-New Zealand island arc, Journal of Volcanic and Geothermal Research 2: pp205-250.

Fleming, C. and Steiner, A. (1951), Sediments beneath Ruapehu Volcano, New Zealand Journal of Science and Technology B32 (6): pp31-32.

Fluche, B. (1983), Geomagnetic and magnetotelluric measurements in the 'Hessische Senke', Journal of Geomagnetism and Geoelectricity 35: pp693-705.

Fuji-ta, K., Ogawa, Y., Ichiki, M., Yamaguchi, S. and Makino, Y. (1999), Audio frequency magnetotelluric survey of Norikura volcano in central Japan, Journal of Volcanology and Geothermal Research 90: pp209-217.

Gamble, T., Goubau, W. and Clarke, J. (1979), Magnetotellurics with a remote magnetic reference, Geophysics 44: pp53-68. Cited in Manzella et al. (2004).

Gerst, A. and Savage, M. (2004), Seismic anisotropy beneath Ruapehu volcano: a possible eruption forecasting tool, Science p. MS no.: RE1103445/CJH/GEOCHEM PHYS.

Grange, L. and Williamson, J. (1930), Tongariro Subdivision, Vol. 10-3, N.Z. Geological Survey 25th Annual Report, n.s.

Grindly, G. (1960), Geological Map of NZ, sheet 8: Taupo, 1:250,000 DSIR, Wellington. 
Groom, R. W. and Bailey, R. C. (1989), Decompostion of magnetotelluric impedance tensors in the presence of local three-dimensional galvanic distortion, Journal of Geophysical Research , B 94: pp1913-1925.

Groom, R. W. and Bailey, R. C. (1991), Analytic investigations of the effects of near-surface three-dimensional galvanic scatterers on MT tensor decompositions, Geophysics 56(4): pp496-518.

Haak, V. (1980), Relations between electrical conductivity and petrological parameters of the crust and upper mantle, Surveys in Geophysics 4: pp57-69. Cited in Müller and Haak (2004).

Hackett, W. (1985), Geology and petrology of Ruapehu volcano and related vents, $\mathrm{PhD}$ thesis, Victoria University of wellington, New Zealand.

Hanekop, O. (2002), Magnetotelluric survey at Mt. Ruapehu: electromagnetic measurements as indicator for volcanic activity? Graduate research project, Victoria University of Wellington, New Zealand.

Hashin, Z. and Shtrikman, S. (1963), A veriational approach to the elastic behaviour of multiphase materials, Journal of Mechanics and Physics of Solids 11: pp127-140.

Hay, R. (1967), Geological Map of NZ, sheet 7: Taranaki, 1:250,000 DSIR, Wellington.

Hermance, J. and Colp, J. (1982), Kilauea Iki lava lake: geophysical constraints on its present (1980) physical state, Journal of Volcanology and Geothermal Research 13: pp31-61.

Horspool, N. (2003), Bending stress and faulting linked to the lead of Ruapehu volcano. Graduate diploma in Science project, Victoria University of Wellington, New Zealand.

Houghton, B., Latter, J. and Hackett, W. (1987), Volcanic hazard assessment for Ruapehu composite volcano, Taupo Volcanic Zone, New Zealand, Bulletin of Volcanology 49: pp737-751.

Houghton, B., Wilson, C., McWilliams, M., Lanphere, M., Weaver, S., Briggs, R. and Pringle, M. (1995), Chronology and dynamics of a large silicic magmatic system: central Taupo Volcanic Zone, New Zealand, Geology 23: pp13-16.

Hurst, A., Bibby, H., Scott, B. and McGuinness, M. (1991), The heat source of Ruapehu Crater Lake; deductions from the energy and mass balances, Journal of Volcanology and Geothermal Research 46: pp1-20.

Hurst, A. and McGinty, P. (1999), Earthquake swarms to the west of Mt Ruapehu preceding its 1995 eruption, Journal of Volcanology and Geothermal Research 90: pp19-28.

Iliceto, V. and Santarato, G. (1999), On the interference of man-made EM fields in the magnetotelluric 'dead band', Geophysical Prospecting 47: pp707-719. 
Ingham, M. (1987), Lower crustal and upper mantle electrical conductivity contrasts in the central North Island of New Zealand, Physics of the Earth and Planetary Interiors 49: pp304-313.

Ingham, M. (1992), Audiomagnetotelluric soundings on White Island volcano, Journal of Volcanology and Geothermal Research 50: pp301-306.

Ingham, M., Whaler, K. and McKnight, D. (2001), Magnetotelluric sounding of the Hikurangi Margin, New Zealand, Geophysics Journal International 144: pp343355.

Jiracek, G. (1990), Near surface and topographic distortions in electromagnetic induction, Surveys in Geophysics 11: pp163-203.

Jones, A. (1988), Static shift of magnetotelluric data and its removal in a sedimentary basin enviroment, Geophysics 53: pp767-778.

Kagiyama, T., Utada, H. and Yamamoto, T. (1999), Magma ascent beneath Unzen volcano, SW Japan, deduced from the electrical resistivity structure, Journal of Volcanology and Geothermal Research 89: pp35-42.

Kao, D. and Orr, D. (1982), Magnetotelluirc studies in the Market Weighton area of eastern England, Geophysical Journal of the Royal Astronomical Society 70: pp323-337.

Kear, D. (1959), Stratigraphy of New Zealand's Cenozoic volcanism northwest of the Volcanic Belt, New Zealand Journal of Geology and Geophysics 2: pp578-589. Cited in Calhaem (1973).

Latter, J. (1981), Volcanic earthquakes, and their relationship to eruptions at Ruapehu and Ngauruhoe volcanoes, Journal of Volcanology and Geothermal Research 9: pp293-309.

Lecointre, J., Neall, V. and Palmer, A. (1998), Quaternary lahar stratigraphy of the western Ruapehu ring plain, New Zealand, New Zealand Journal of Geology and Geophysics 41: pp225-245.

Lilley, F. (1998), Magnetotelluric tensor decomposition: part1, theory for a basic procedure, Geophysics 63: pp1885-1897.

Luther, G. (1999), The geology, petrology, geochemisrty and tephrochronology of south-southwest Mt Ruapehu, New Zealand. Honours thesis, Victoria University of Welington, New Zealand.

Mackie, R. and Madden, T. R. (1993), Three-dimensional magnetotelluric inversion using conjugate gradients, Geophysics Journal International 115: pp215-229.

Manzella, A., Volpi, G., Zaja, A. and Meju, M. (2004), Combined TEM-MT investigation of shallow-depth resistivity structure of Mt. Somma Vesuvius, Journal of Volcanology and Geothermal Research 131: pp19-32. 
Marriott, M. (1969), A magnetotelluric investigation of the North Island Volcanic Plateau, Master's thesis, Victoria University of Wellington, New Zealand.

Matsushima, N., Oshima, H., Ogawa, Y., Takakura, S., Satoh, H., Utsugi, M. and Nishida, Y. (2001), Magma prospecting in Usu volcano, Hokkaido, Japan, using magnetotelluric soundings, Journal of Volcanology and Geothermal Research 109: pp263-277.

McLaughin, C., Ingham, M., Whaler, K. and McKnight, D. (2002), A magnetotelluric transect of the Wairarapa region, New Zealand, New Zealand Journal of Geology and Geophysics 45: pp257-269.

Miller, V. and Savage, M. (2001), Changes in seismic anisotropy after volcanic eruptions: evidence from Mount Ruapehu, Science 293: pp2231-2233.

Mogi, T. and Nakama, S. (1993), Magnetotelluric interpretation of the geothermal system of the Kuju volcano, southwest Japan, Journal of Volcanology and Geothermal Research 56: pp297-308.

Mozley, E., Goldstein, N. and Morrison, H. (1986), Magnetotelluric investigations at Mount Hood, Oregon, Journal of Geophysical Research 91: pp11,596-11,610.

Müller, A. and Haak, V. (2004), 3-D modelling of the deep electrical conductivity of Merapi volcano (Central Java): Integrating magnetotellurics, induction vectors and the effect of steep topography, GeoForschungsZentrum, Germany p. DOI:10.1016/J.Juolgeores.2004.05.023.

Newman, G. and Alumbaugh, D. (2000), Three-dimensional magnetotelluric inversion using non-linear conjugate gradients, Geophysics Journal International 140: $p$ 410-424.

Niblett, E. and Sayn-Wittgenstein, C. (1960), Variation of electrical conductivity with depth by the magnetotelluric method, Geophysics 25: pp998-1008. Cited in Ingham at al. (2001).

Ogawa, Y., Matsushima, N., Oshima, H., Takakura, S., Utsugi, M., Hirano, K., Igarashi, M. and Doi, T. (1998), A resistivity cross-section of Usu volcano, Hokkaido, Japan, by audiomagnetotelluric soundings, Earth, Planets and Space 50: pp339-346.

Olson, J. (1985), Seismicity and seismic structure of the Tongariro Volcanic Centre, Master's thesis, Victoria University of Wellington, New Zealand.

Park, S. and Torres-Verdin, C. (1988), A systematic approach to the interpretation of magnetotelluric data in volcanic enviroments with applications to the quest for magma in Long Valley, California, Journal of Geophysical Research 93: pp13,26513,283 .

Parker, R. (1980), The inverse problem of electromagnetic induction: existence and construction of solutions based upon incomplete data, Journal of Geophysical Research 85: pp4421-4428. 
Pulford, A. and Stern, T. (2004), Pliocene exhumation and landscape evolution of central North Island, New Zealand: the role of the upper mantle, Journal of Geophysical Research 109: F01016, doi:10.1029/2003JF000046.

Ranganayaki, R. (1984), An interpretive analysis of magnetotelluric data, Geophysics 49: $p$ 1730-1748.

Risk, G., Caldwell, T. and Bibby, H. (1994), Deep resistivity surveys in the WaiotapaWaikite-Raporoa region, New Zealand, 2(2): pp287-294.

Rodi, W. and Mackie, R. (2001), Nonlinear conjugate gradients algorithm for 2-D magnetotelluric inversion, Geophysics 66: pp174-187.

Schilling, F., Partzsch, G., Brasse, H. and Schwarz, G. (1997), Partial melting below the magmatic arc in the central Andes deduced from geoelectromagnetic field experiments and laboratory data, Physics of the Earth and Planetary Interiors 103: $p$ 17-31.

Sissons, B. and Dibble, R. (1981), A seismic refraction experiment southeast of Ruapehu volcano, New Zealand Journal of Geology and Geophysics 24: pp31-38.

Smith, E., Stern, T. and Reyners, M. (1989), Subduction and back-arc activity at the Hikurangi convergent margin, New Zeland, Pure and Applied Geophysics 129(1/2): pp203-231.

Smith, J. and Booker, J. (1991), Rapid inversion of two and three-dimensional magnetotelluric data, Journal of Geophysical Research 96: pp3905-3922.

Stern, T. and Davey, F. (1987), A seismic investigation of the crustal and upper mantle structure within the Central Volcanic Region of New Zealand, New Zealand Journal of Geology and Geophysics 30: pp217-231.

Stipp, J. (1968), The geochronology and petrogenesis of the Cenozoic volcanics of the North Island, New Zealand, PhD thesis, Australian National University, Australia. Cited in Zeng (1996).

Stratford, W. and Stern, T. (2004), Strong seismic reflections and melts in the mantle of a continental back-arc basin, Geophysical Research Letters 31: Lo6622, doi10.1029/2003GL019232.

Swift, C. (1967), A magnetotelluric investigation of an electrical conductivity anomaly in the southwestern United States, PhD thesis, Massachusetts Institute of Technology, Massachusetts. Cited in Fluche (1983).

Topping, W. (1974), Some aspects of Quaternary history of Tongariro Volcanic Centre, PhD thesis, Victoria University of Wellington, New Zealand.

Villamor, P., Van Dissen, R., Alloway, B., Palmer, A. and Litchfield, N. (2004), Temporal variability of earthquake occurance and slip rate on the Rangipo Fault, Taupo Rift, New Zealand. 
Weaver, J., Agarwal, A. and Lilley, F. (2000), Characterization of the magnetotelluric tensor in terms of its invariants, Geophysical Journal International 141: pp321336.

Wessel, P. and Smith, W. (2001), The generic mapping tools GMT version 3.4: Technical reference and cookbook.

Wilson, C., Houghton, B., McWilliams, M., Lanphere, M., Weaver, S. and Briggs, R. (1995), Volcanic and structural evolution of Taupo Volcanic Zone, New Zealand: a review, Journal of Volcanology and Geothermal Research 68: pp1-28.

Zeng, Y. (1996), Geophysical investigations of the subsurface structure of Tongariro volcanic centre, New Zealand, PhD thesis, Victoria University of Wellington, New Zealand.

GEO (2004), A guide to using WinGLink(R) version 2.04.05.

Geonet (2004), Internet http://data.geonet.org.nz/QuakeSearch/index.jsp. Last modified 19 March 2004.

Mat (1992), MATLAB Reference Guide.

National Enviromental Satellite Data and Information Services (2004), Internet http://www.ngdc.noaa.gov/stp/GEOMAG/dst.html. Last modified 26 Feb 2004.

SEG (1991), MT/EMAP data interchange standard. 


\section{UNDISTORTED DATA}

This appendix presents the data collected from each site individually. The data can be summarised by five graphs that show the sites properties over the period range. These graphs are:

1. Apparent resistivity after distortion has been removed

2. Phase

3. Ellipticity

4. Beta value

5. Strike

and have been included here for completeness and so that the conclusions reached in this thesis can be verified. 

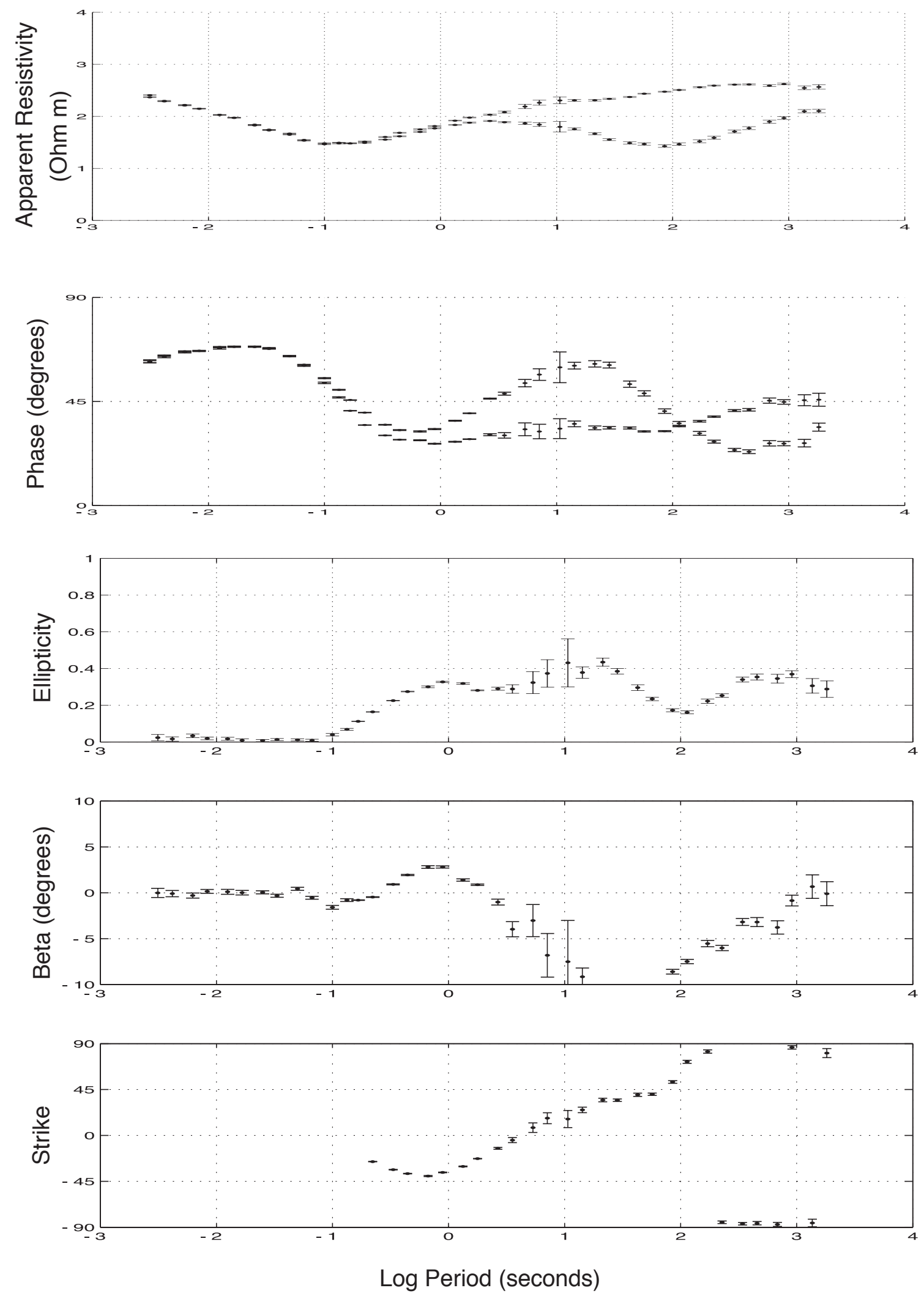

Figure A.1 Data from site MTR-101a 

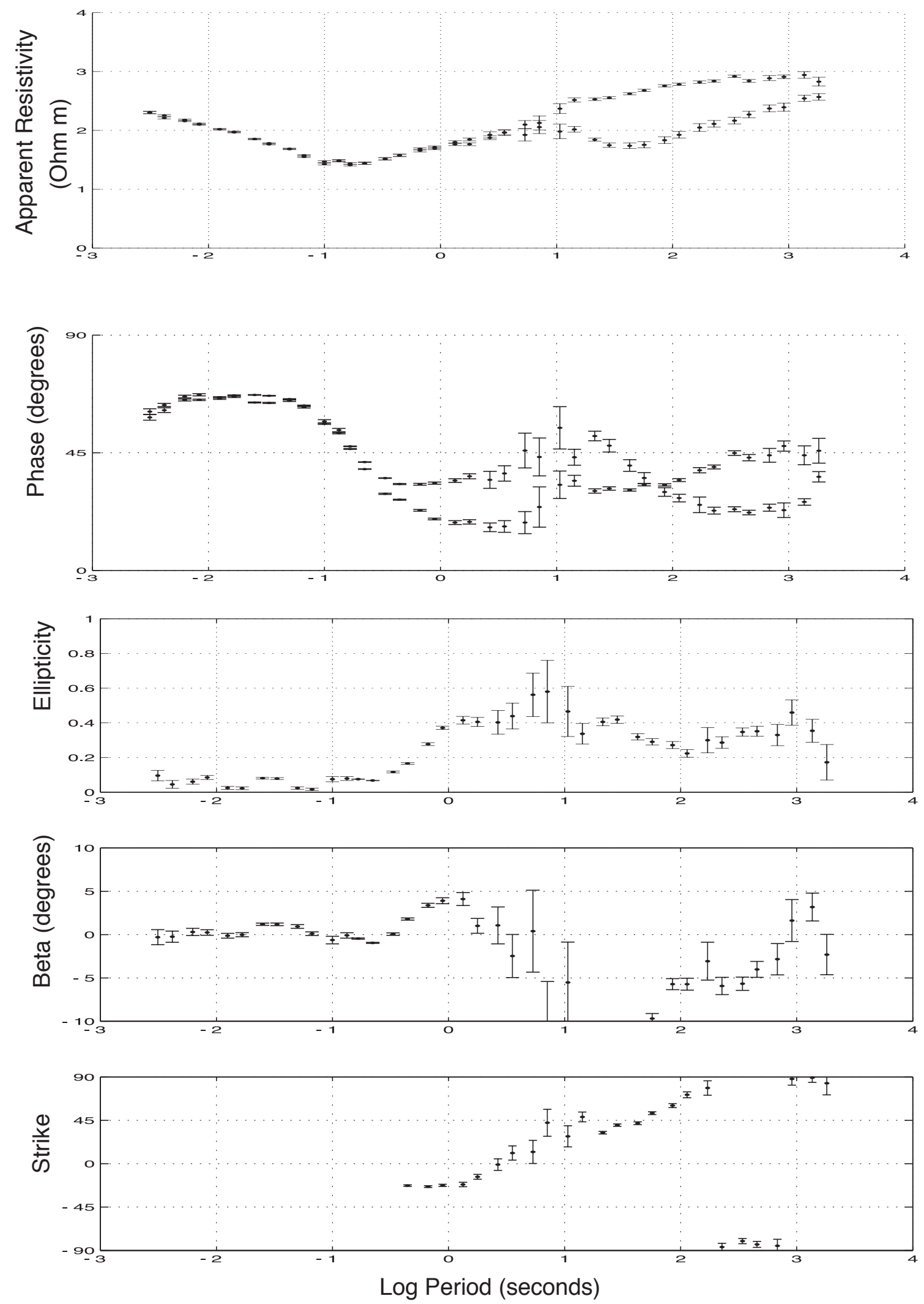

Figure A.2 Data from site MTR-102a 

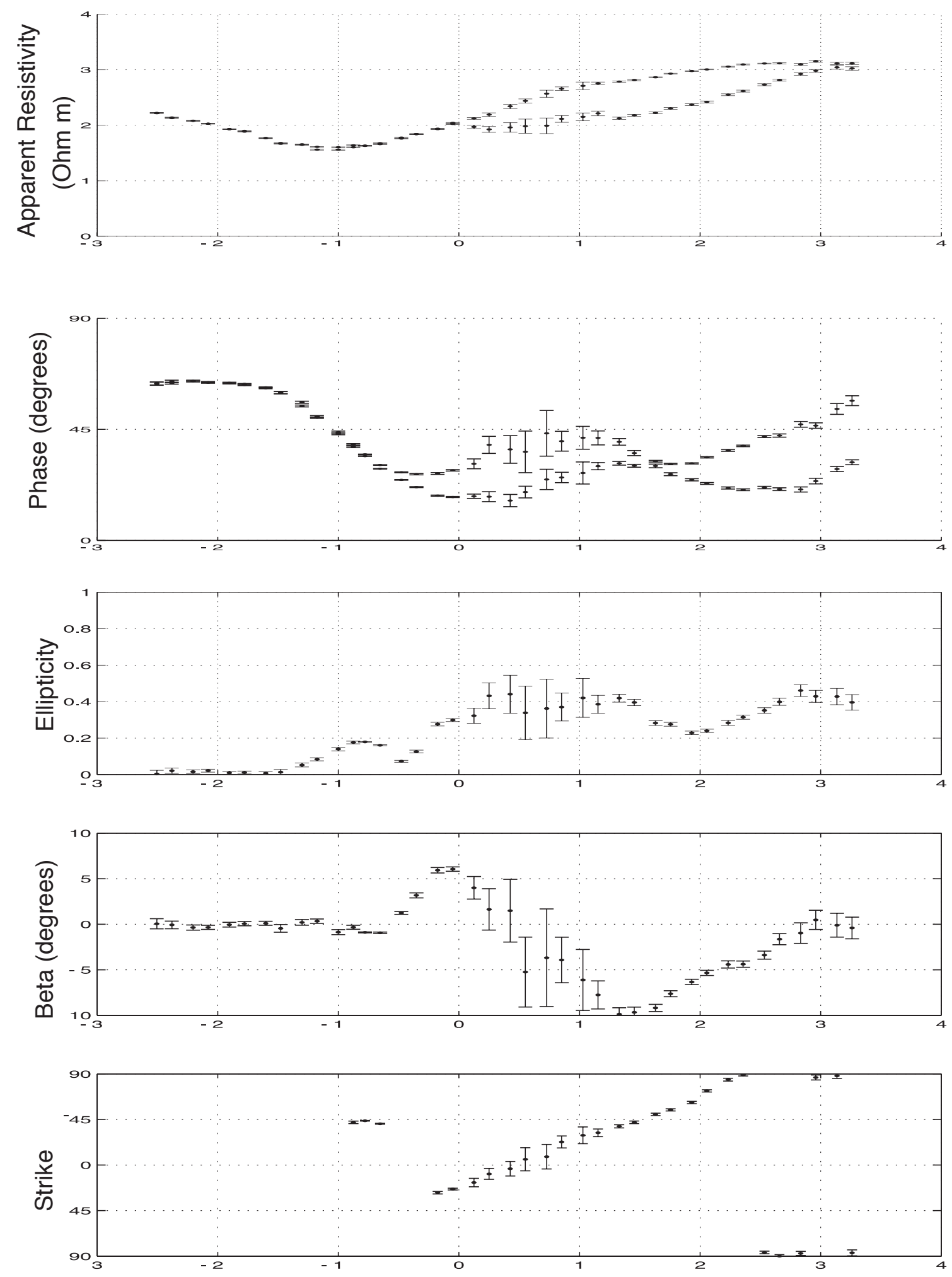

Log Period (seconds)

Figure A.3 Data from site MTR-103a 

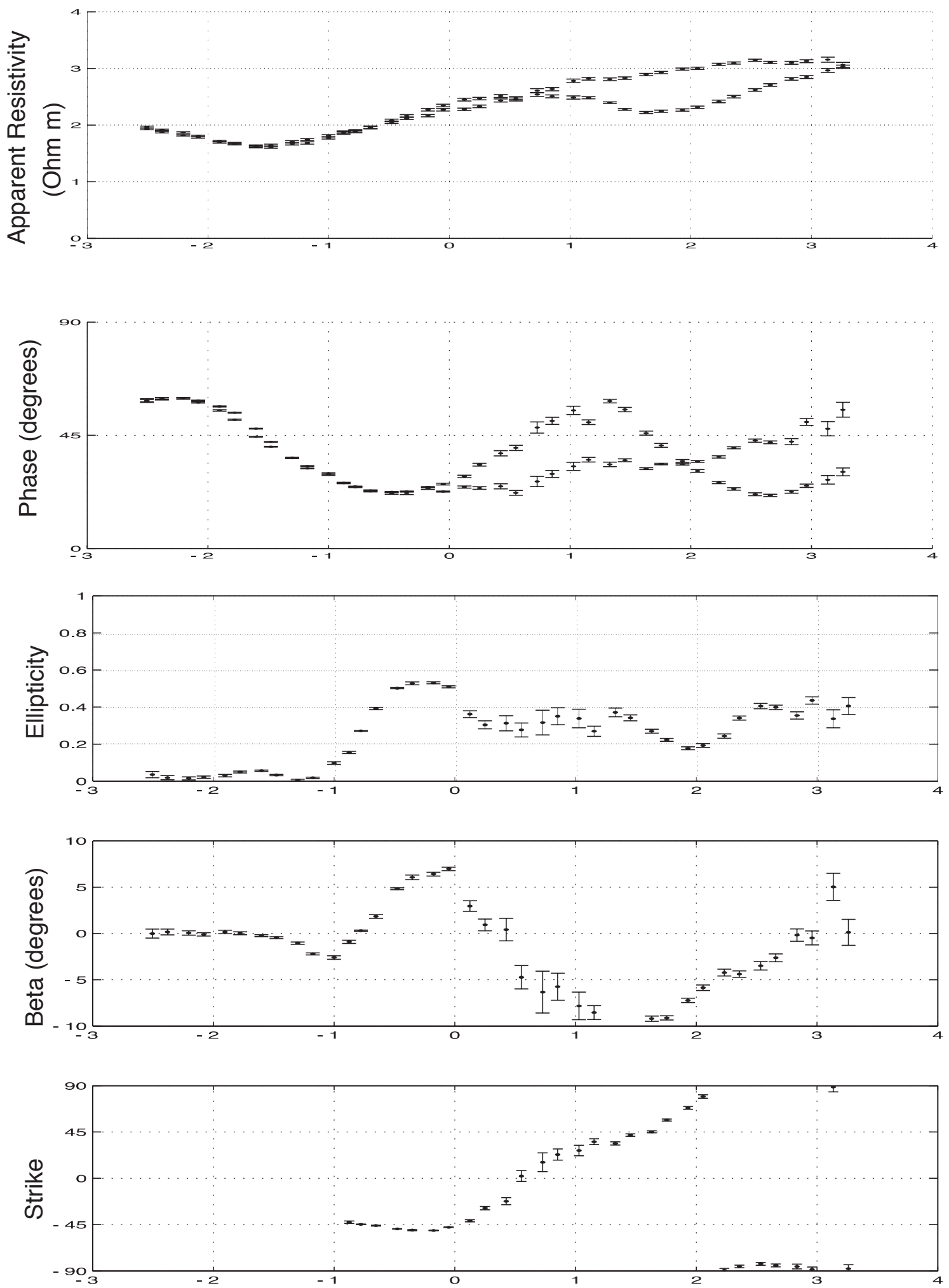

Log Period (seconds)

Figure A.4 Data from site MTR-104a 

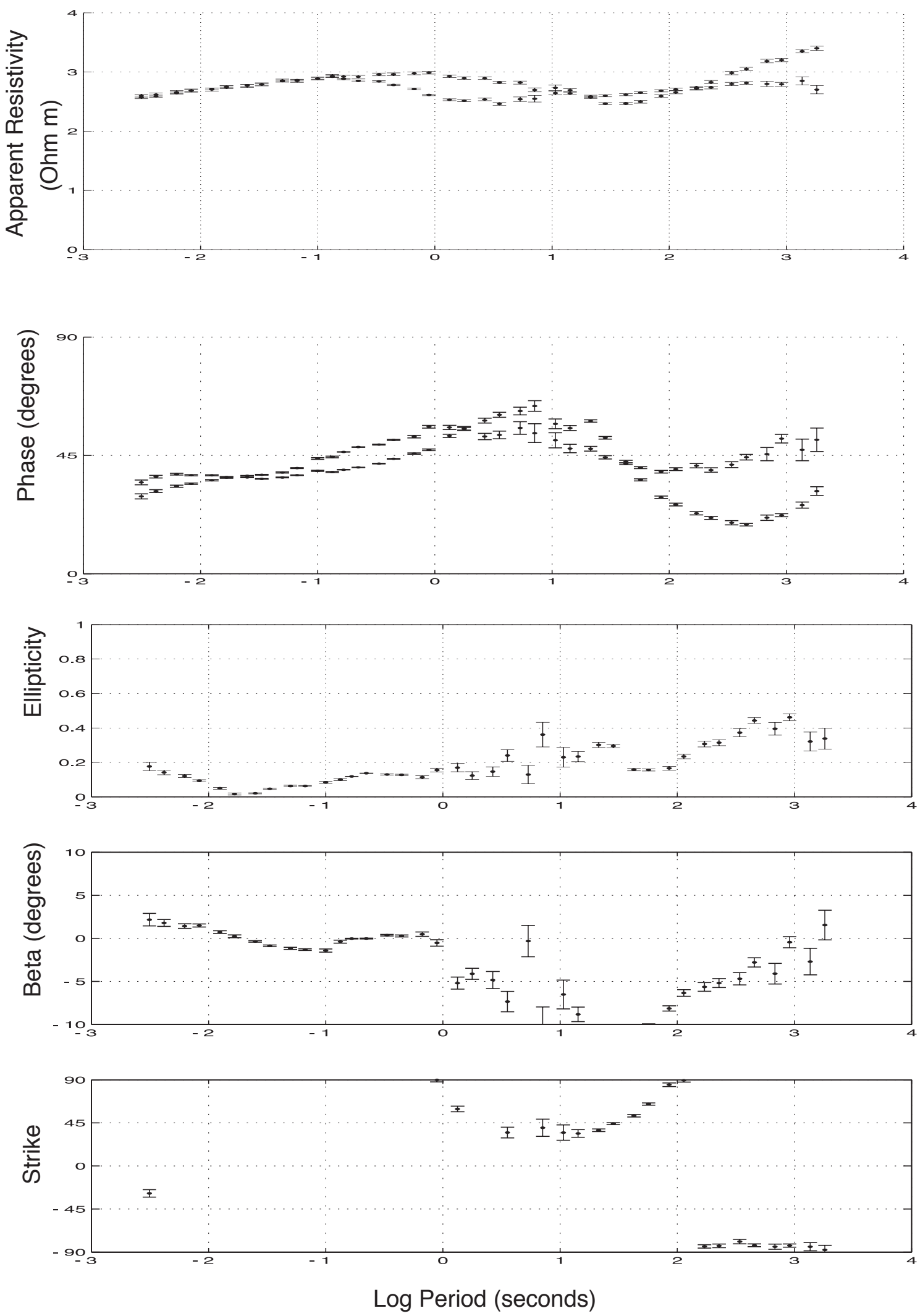

Figure A.5 Data from site MTR-105a 

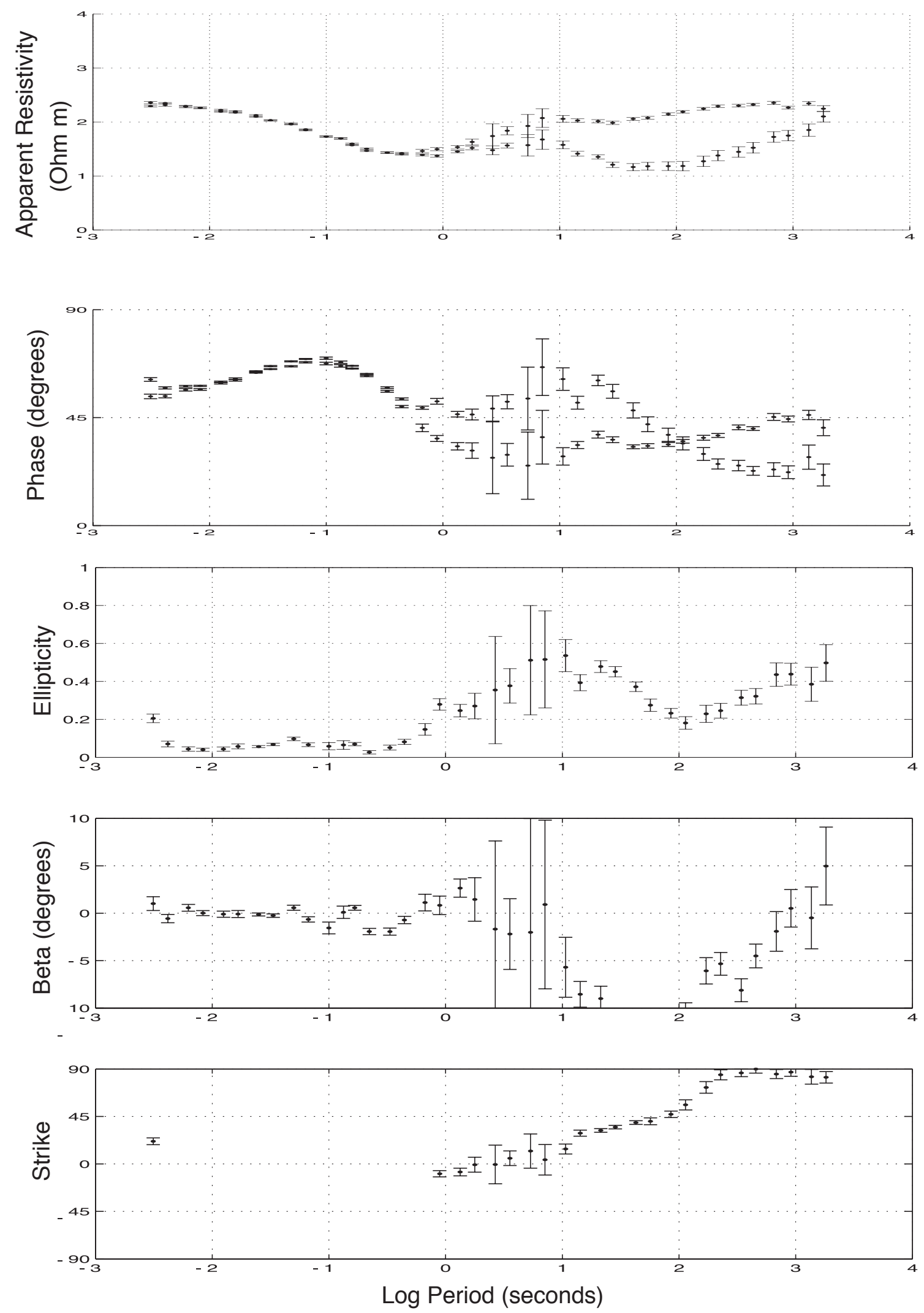

Figure A.6 Data from site MTR-106a 

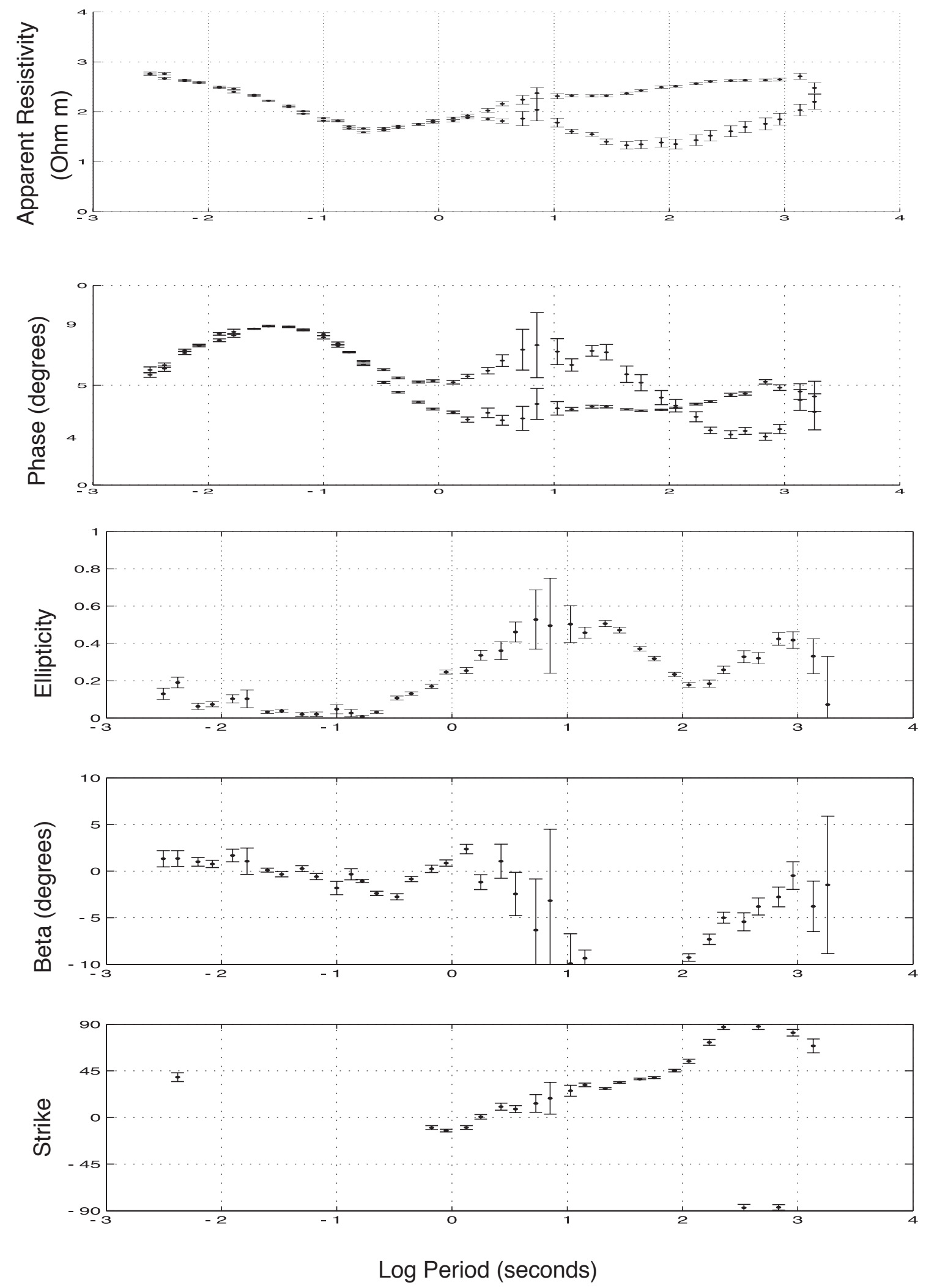

Figure A.7 Data from site MTR-107a 

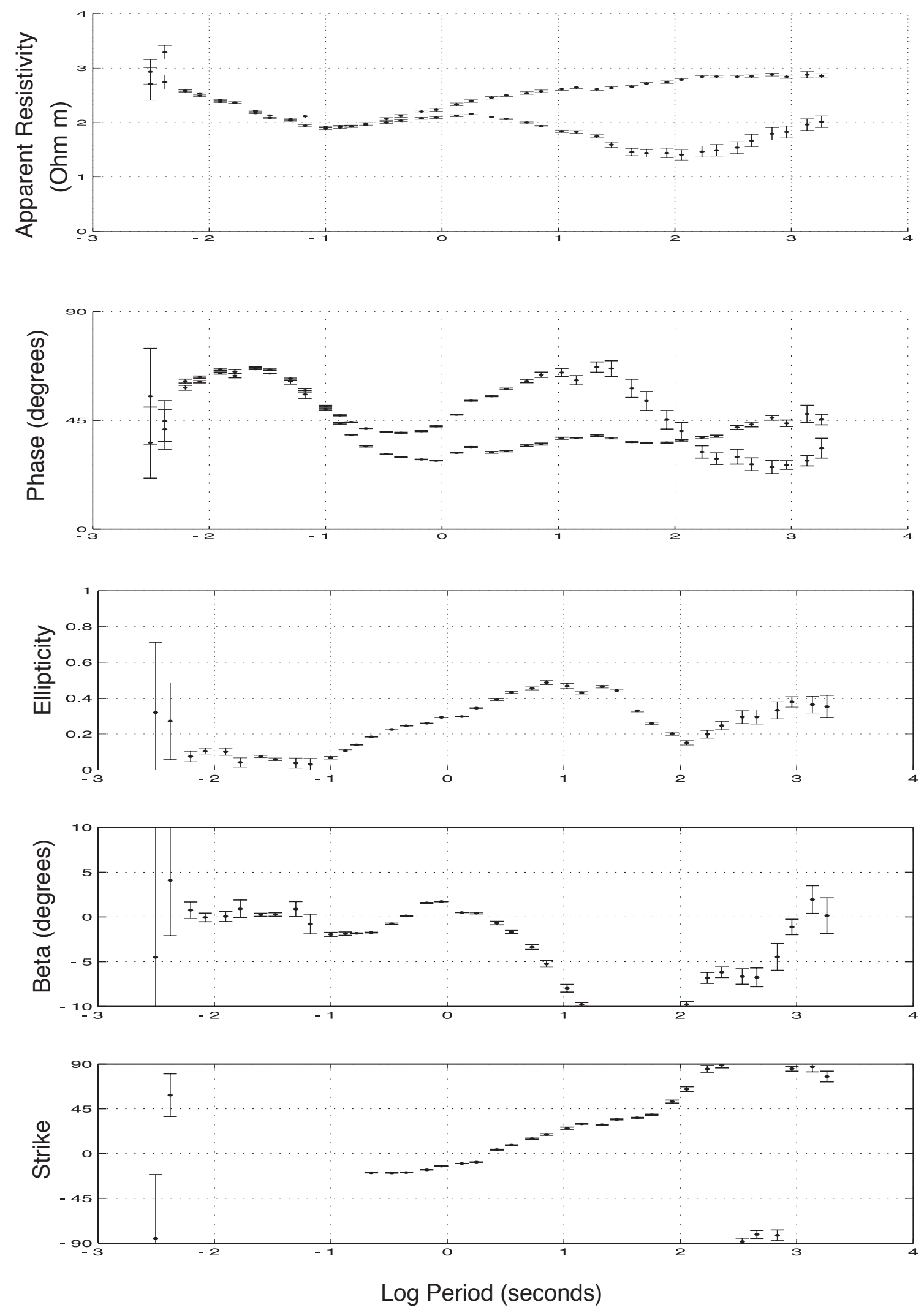

Figure A.8 Data from site MTR-110a 

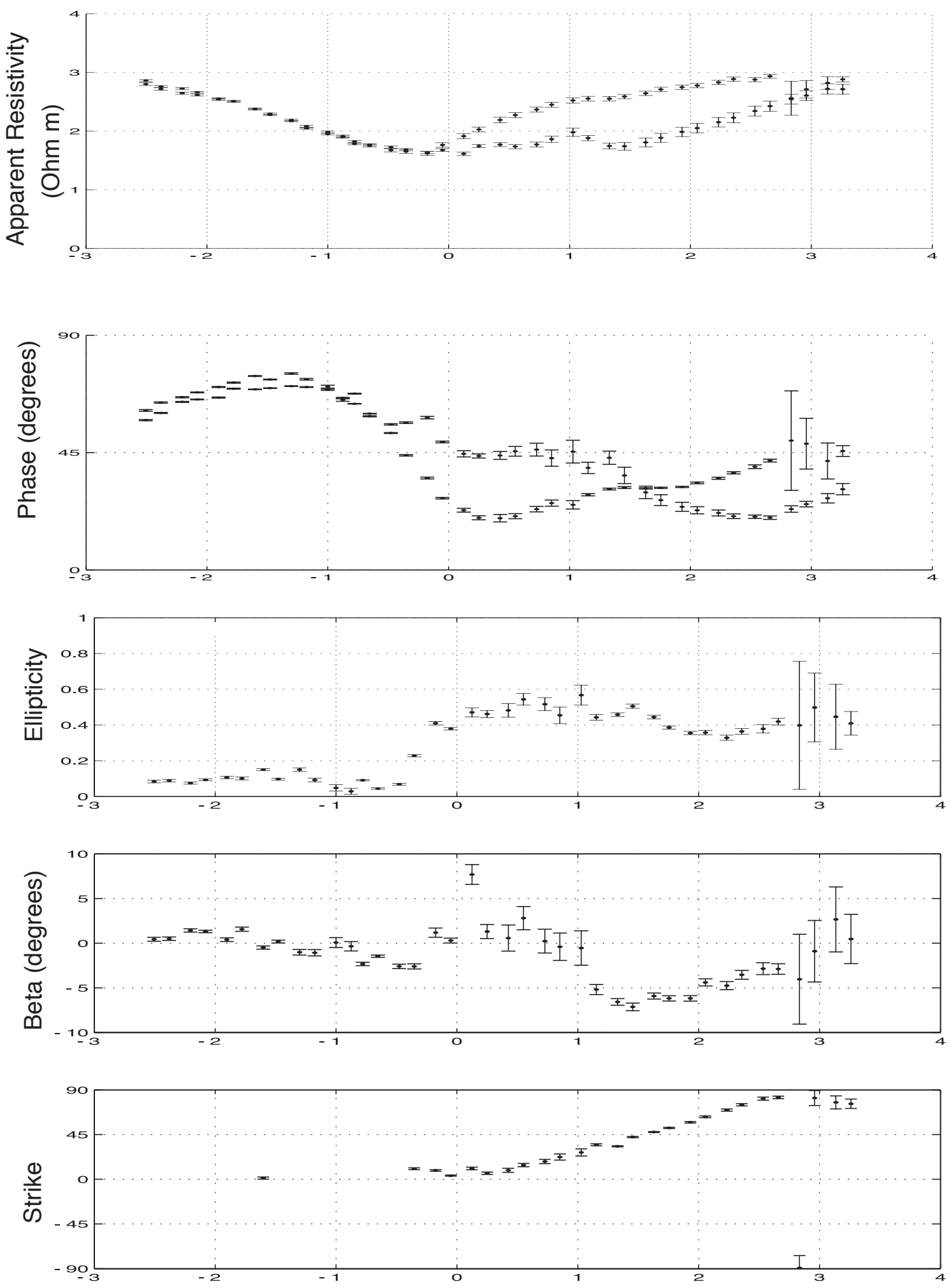

Log Period (seconds)

Figure A.9 Data from site MTR-111a 

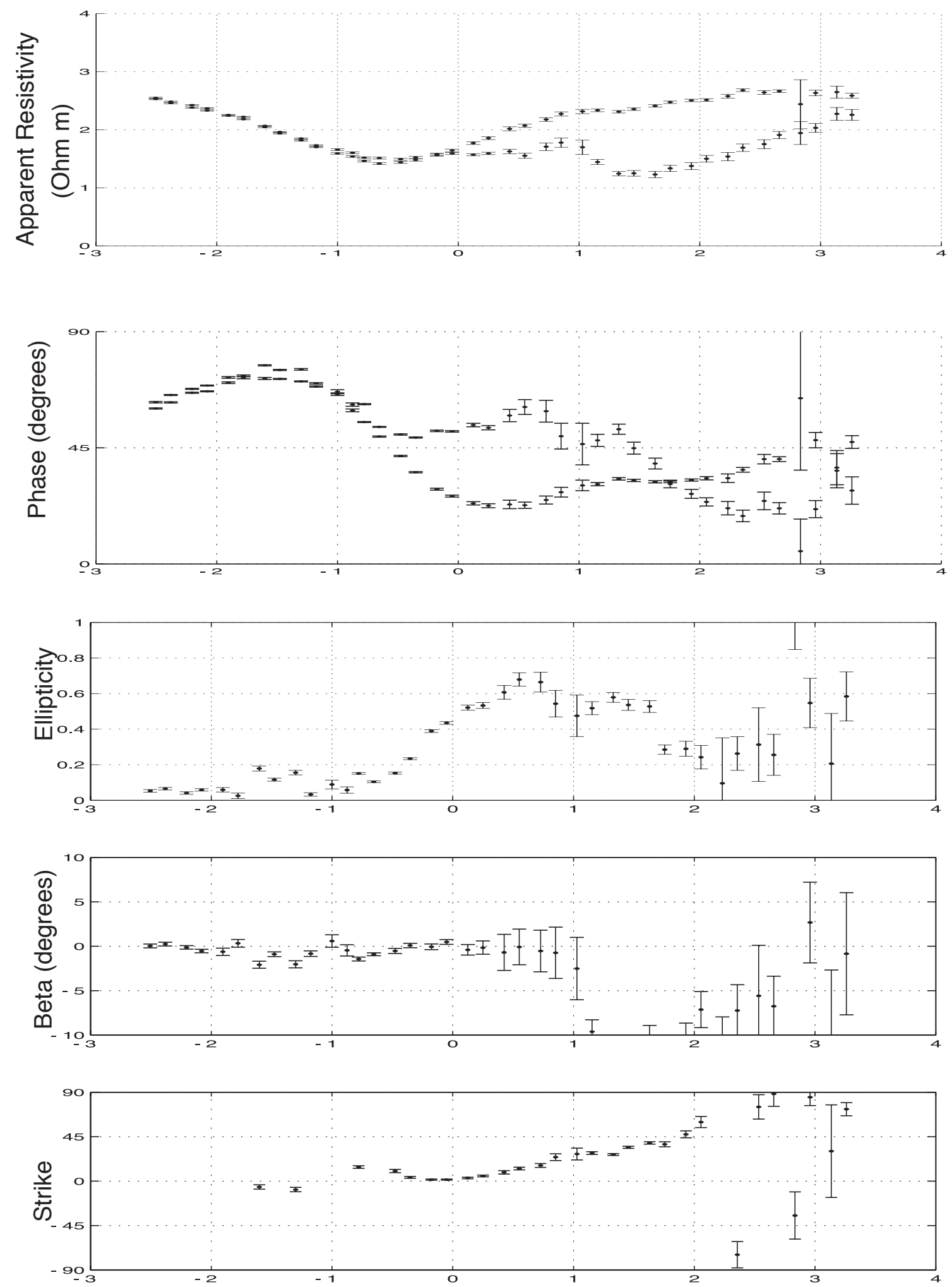

Log Period (seconds)

Figure A.10 Data from site MTR-112a 

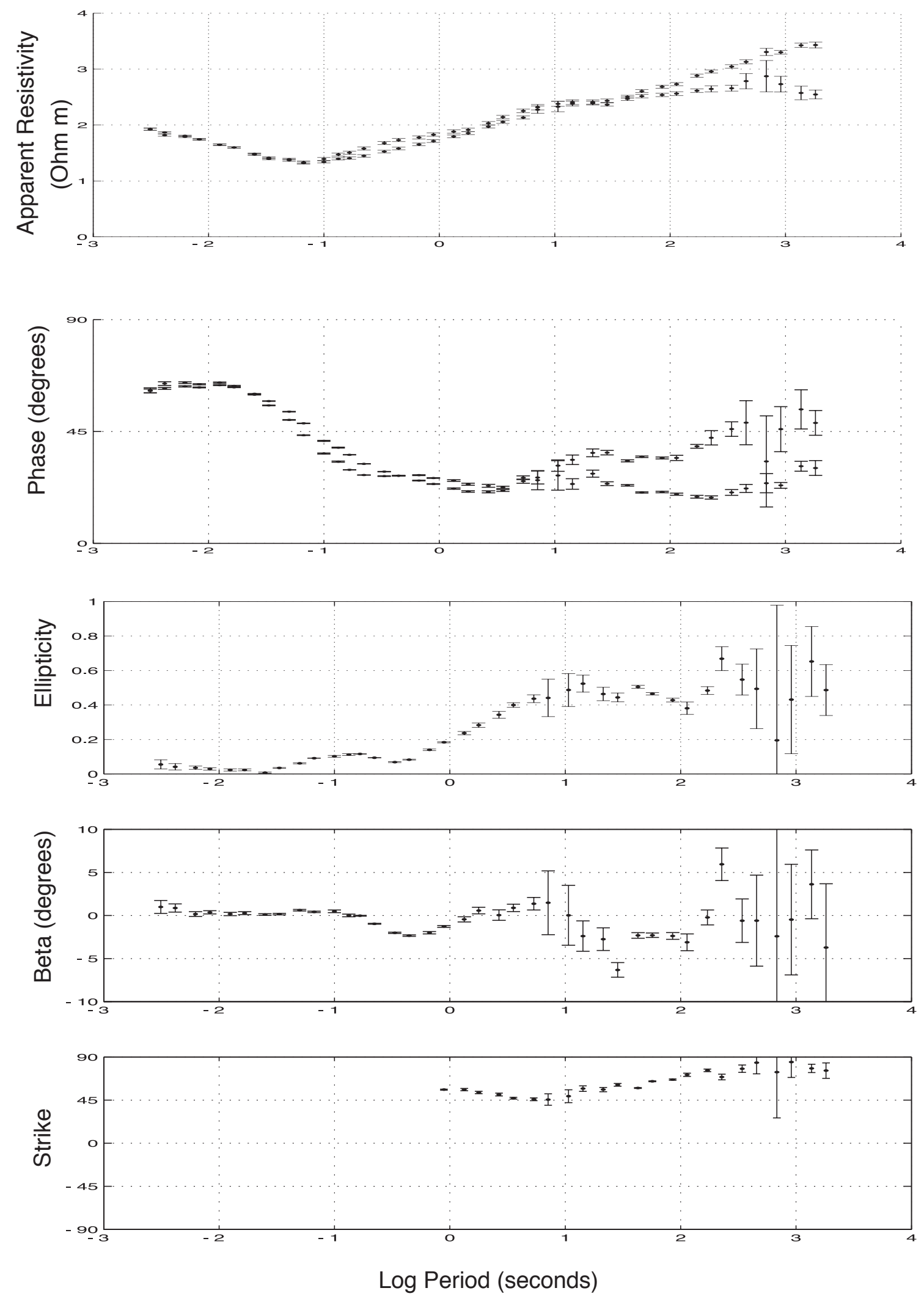

Figure A.11 Data from site MTR-113a 

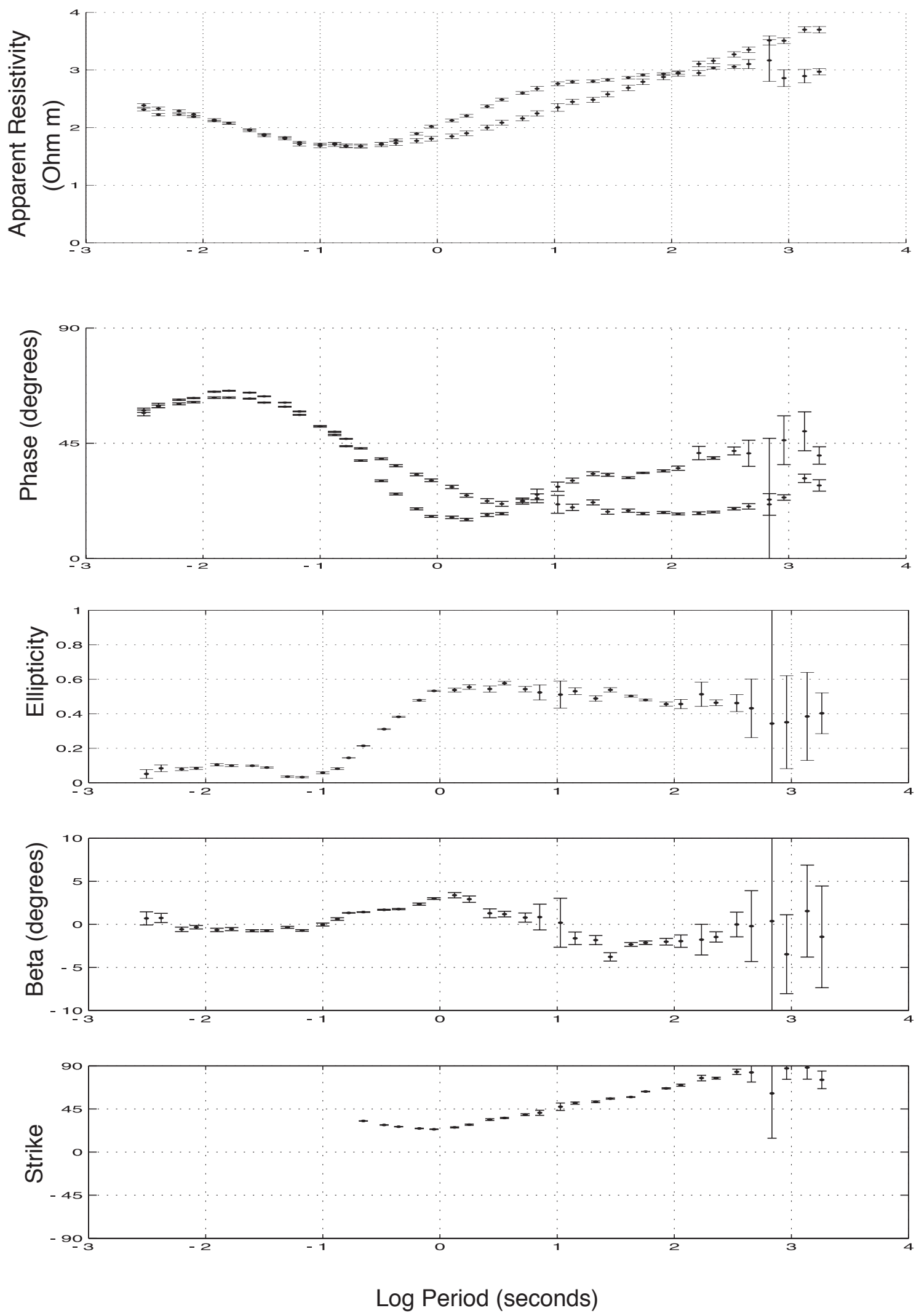

Figure A.12 Data from site MTR-114a 

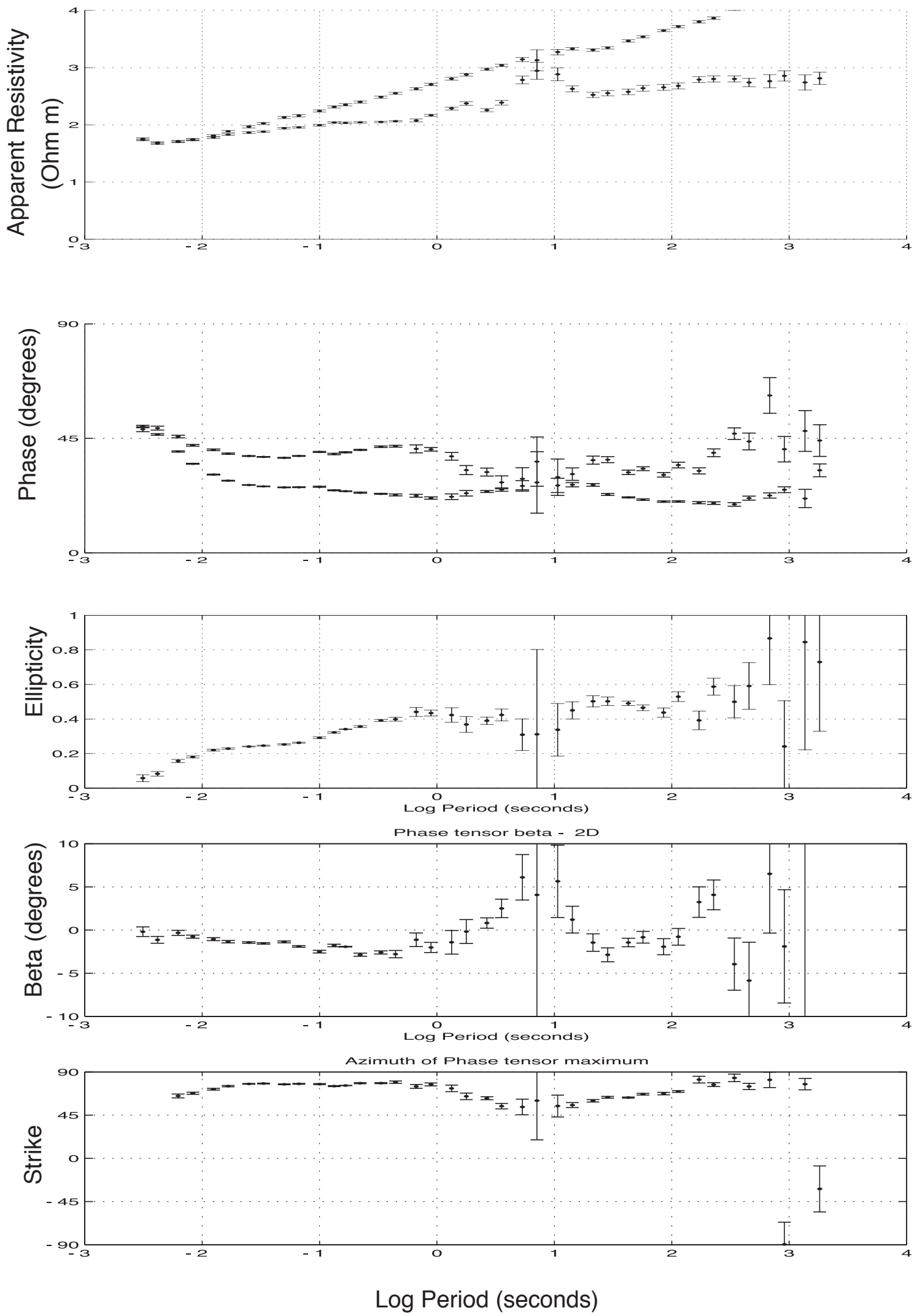

Figure A.13 Data from site MTR-115a 

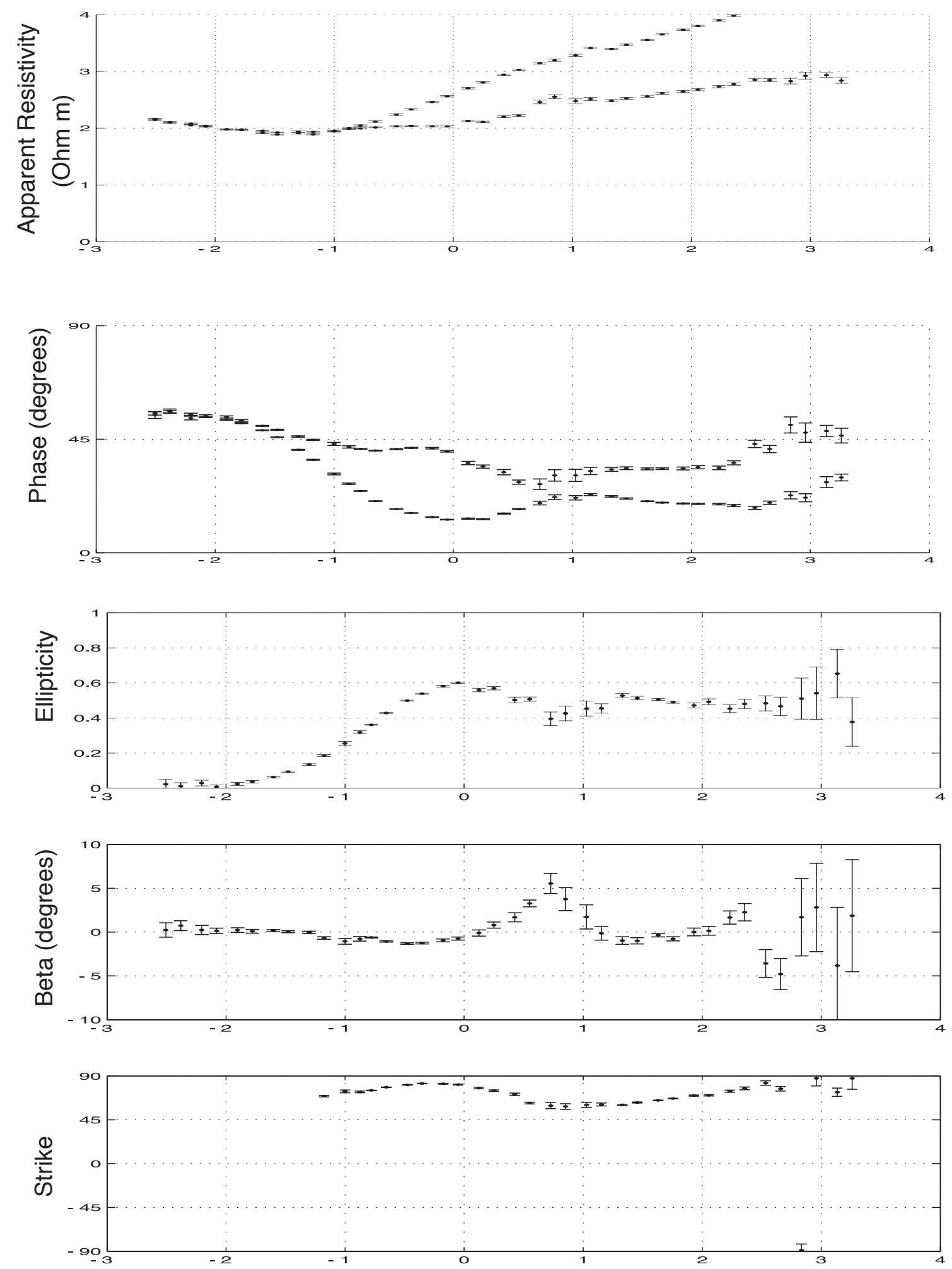

Log Period (seconds)

Figure A.14 Data from site MTR-116a 

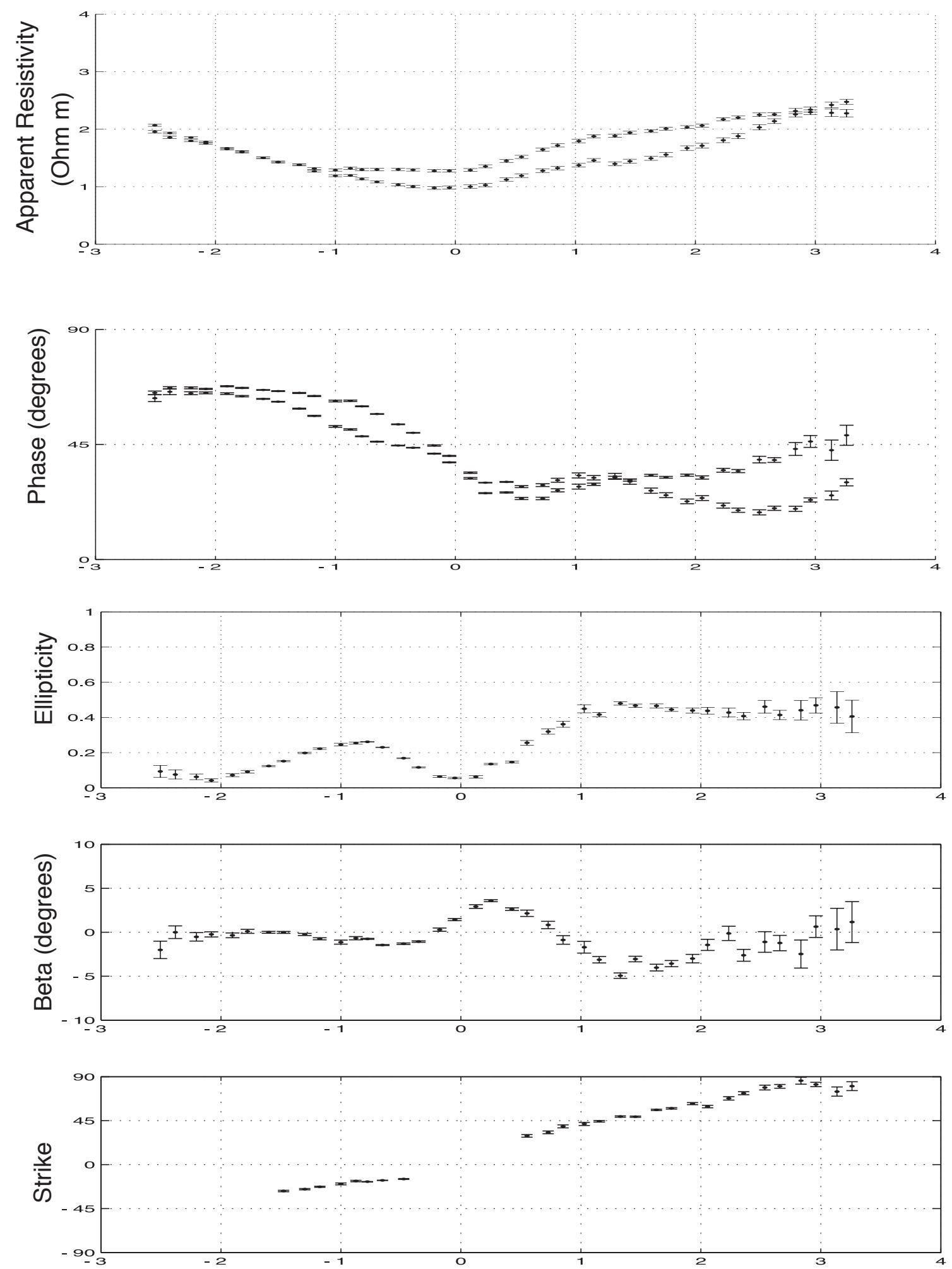

Log Period (seconds)

Figure A.15 Data from site MTR-117a 

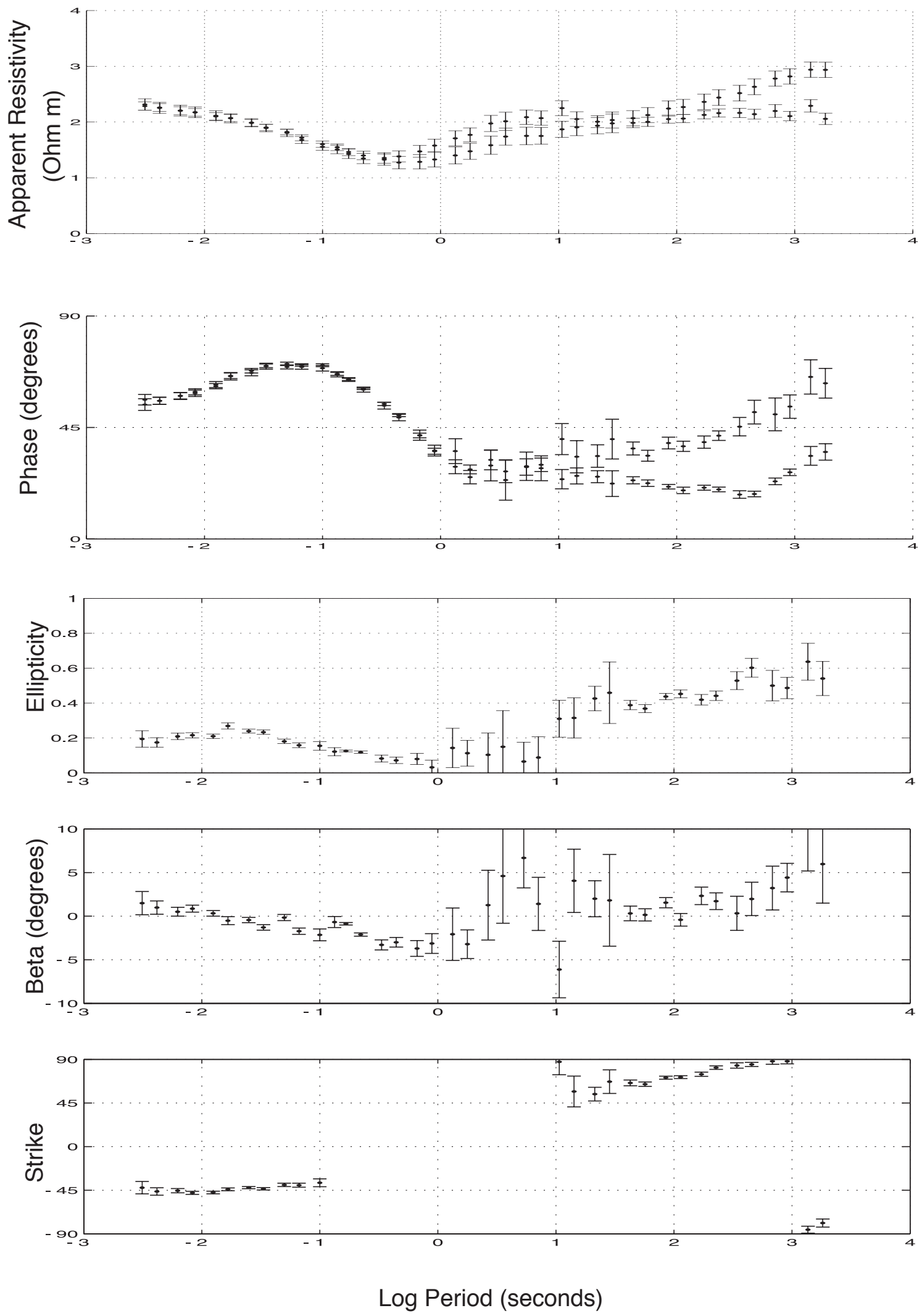

Figure A.16 Data from site MTR-118b 

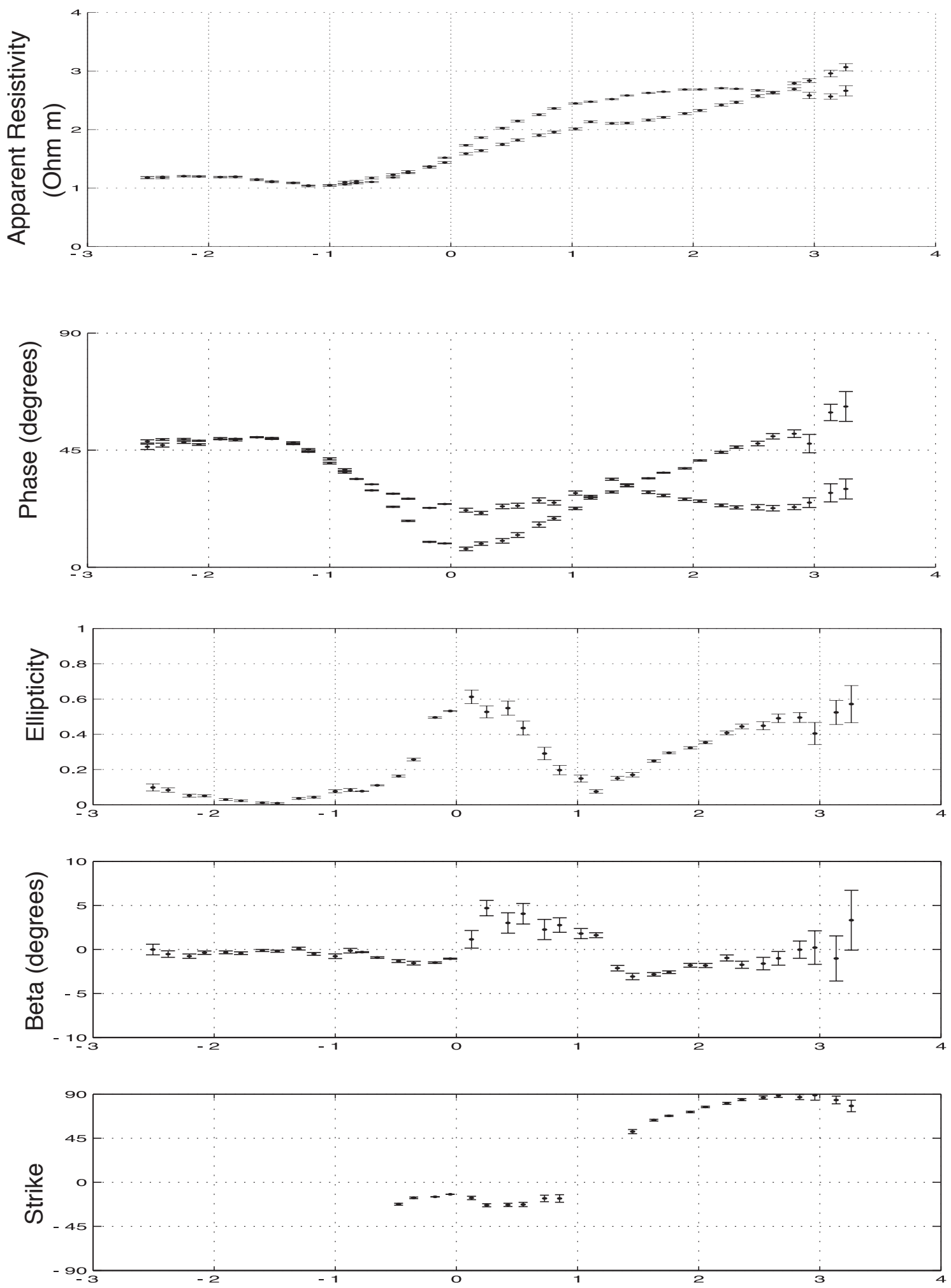

Log Period (seconds)

Figure A.17 Data from site MTR-119a 

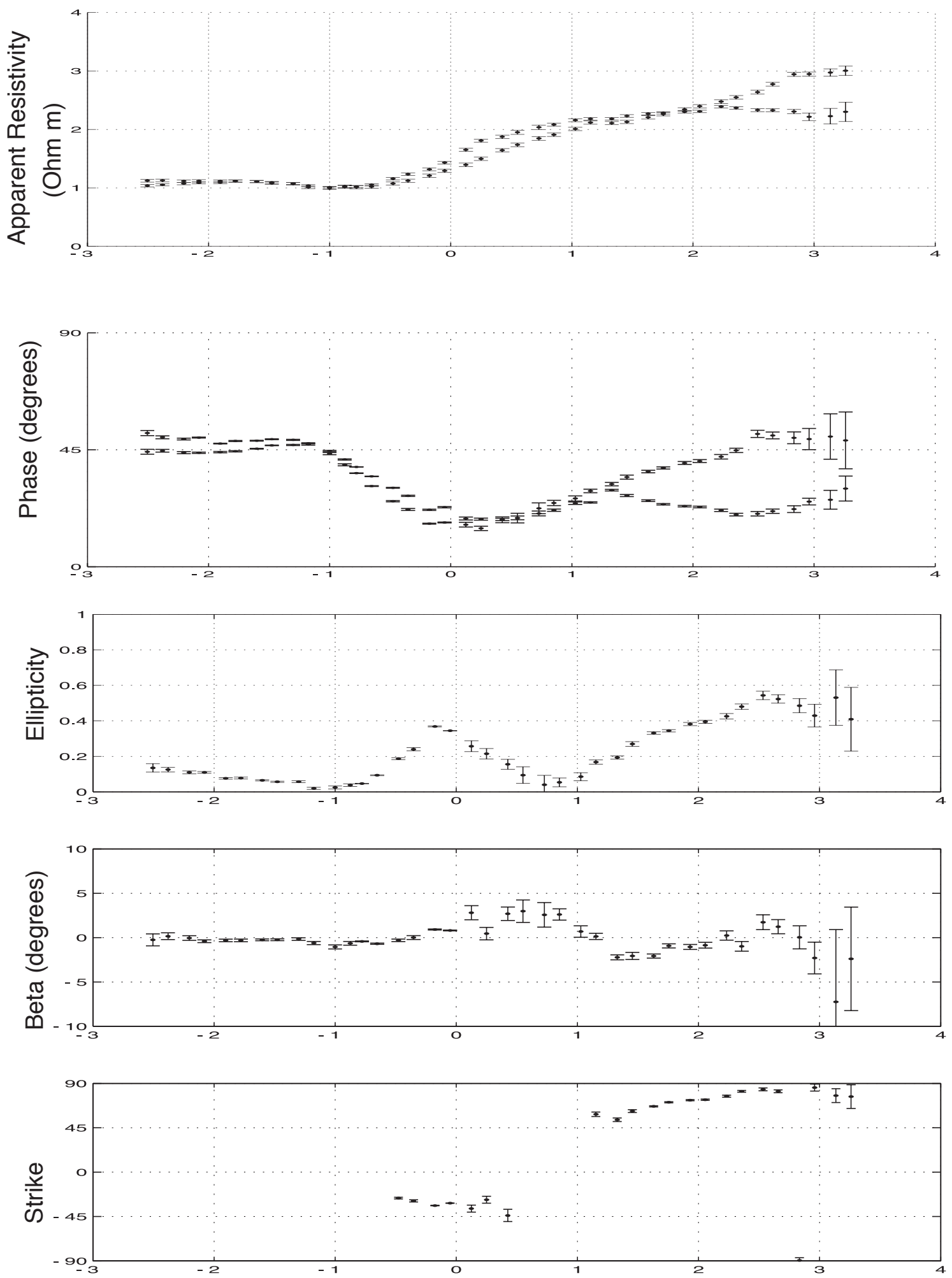

Log Period (seconds)

Figure A.18 Data from site MTR-120a 

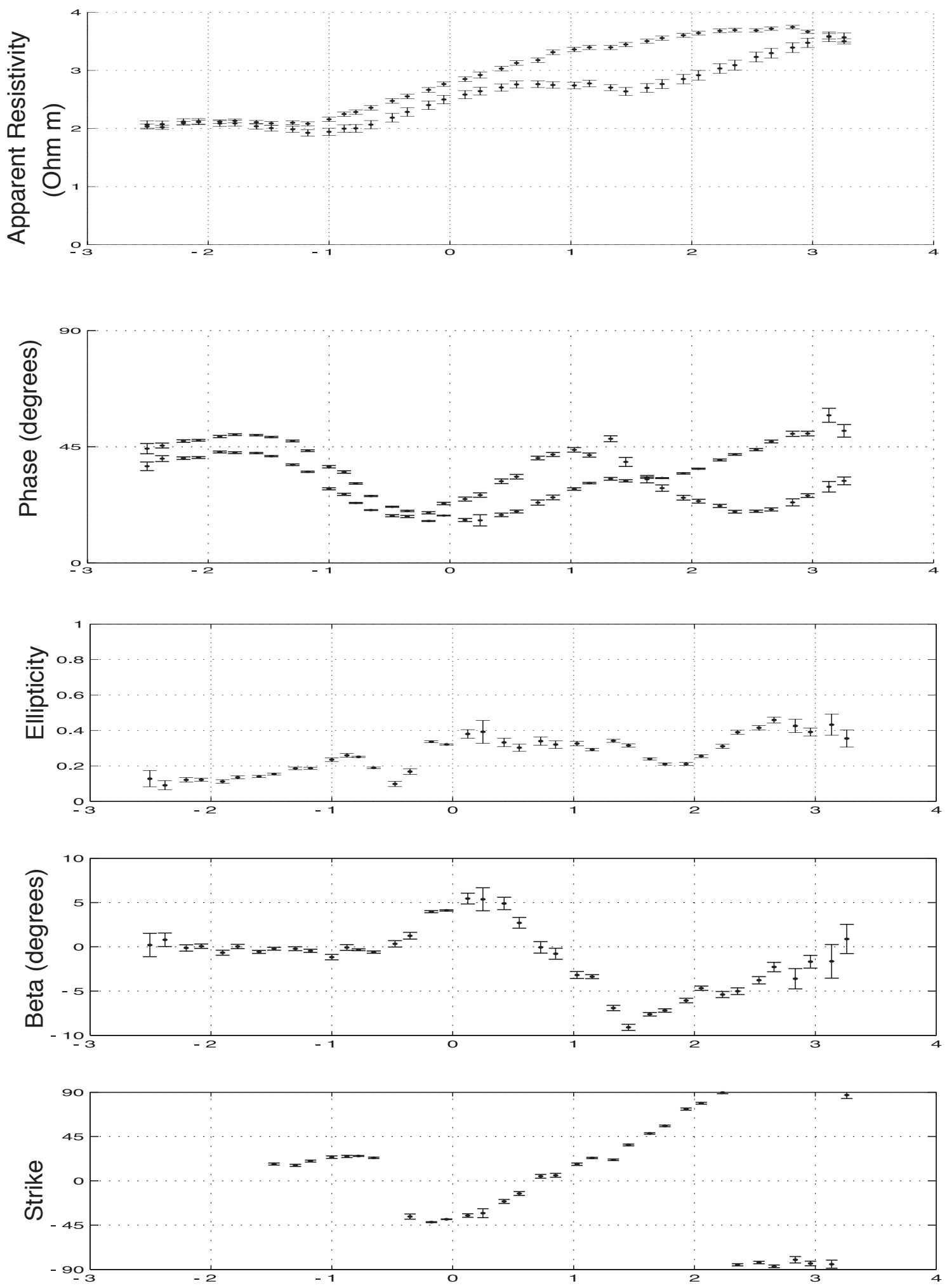

Log Period (seconds)

Figure A.19 Data from site MTR-121a 

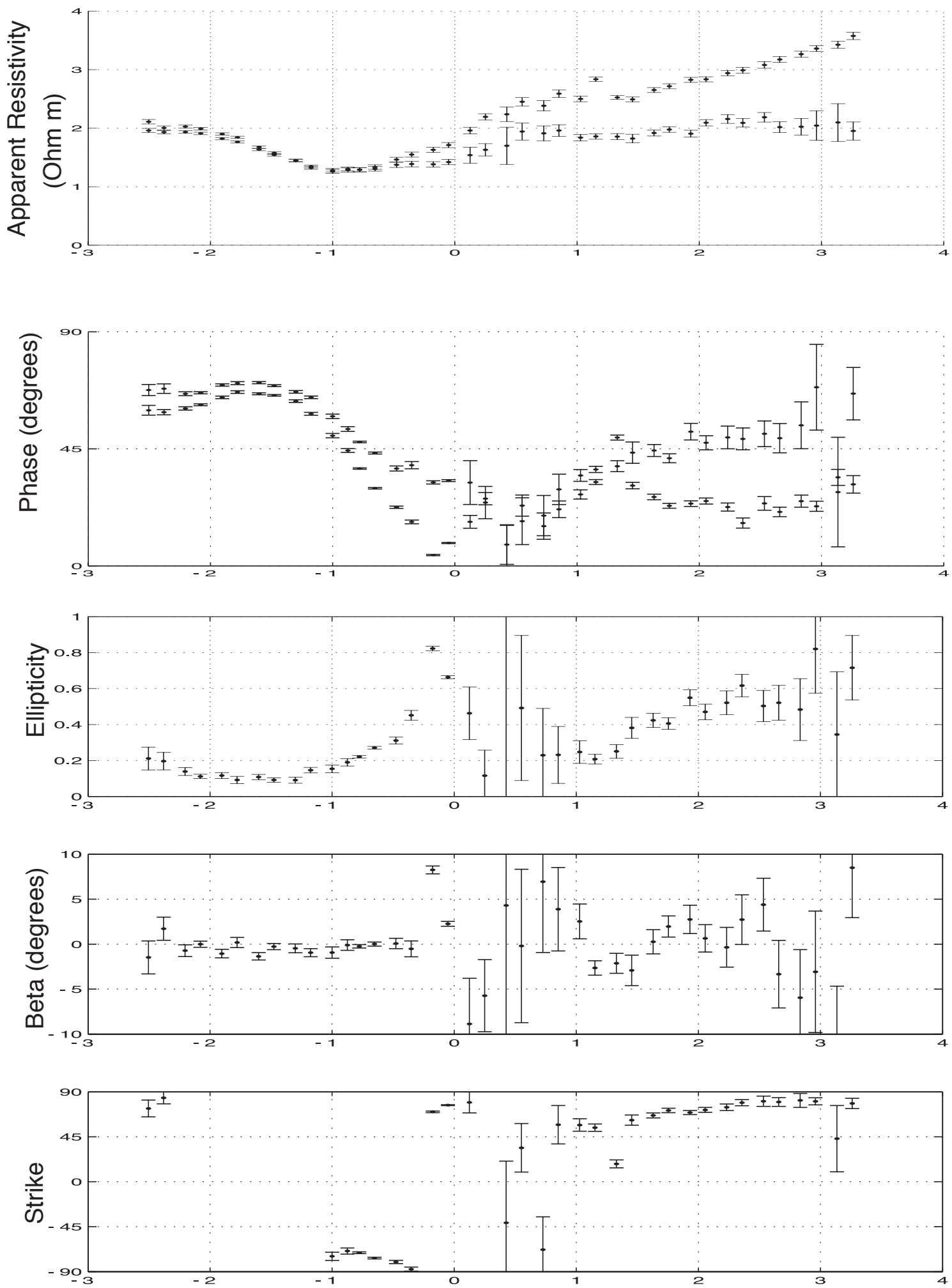

Log Period (seconds)

Figure A.20 Data from site MTR-122a 

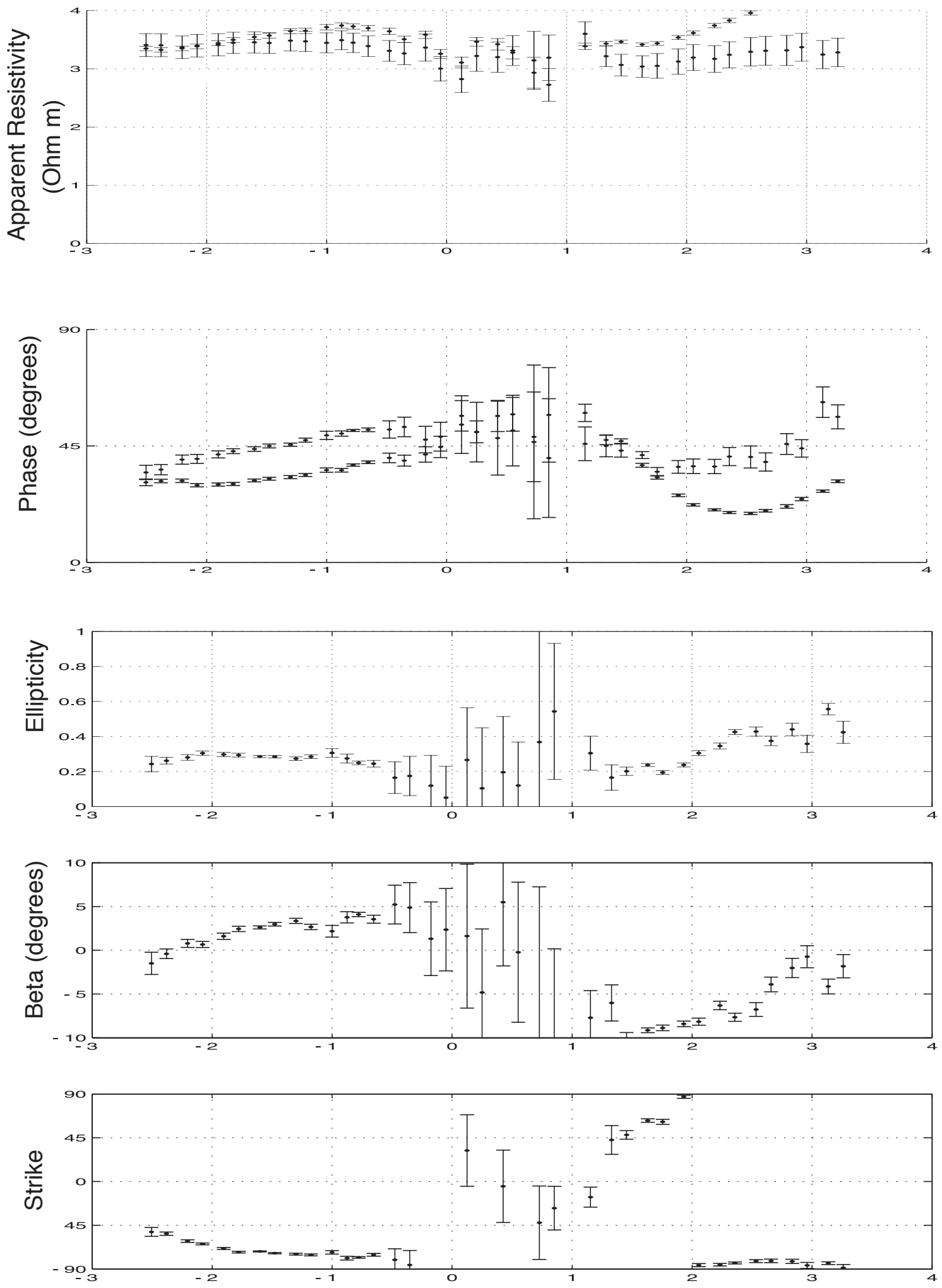

Log Period (seconds)

Figure A.21 Data from site MTR-123a 

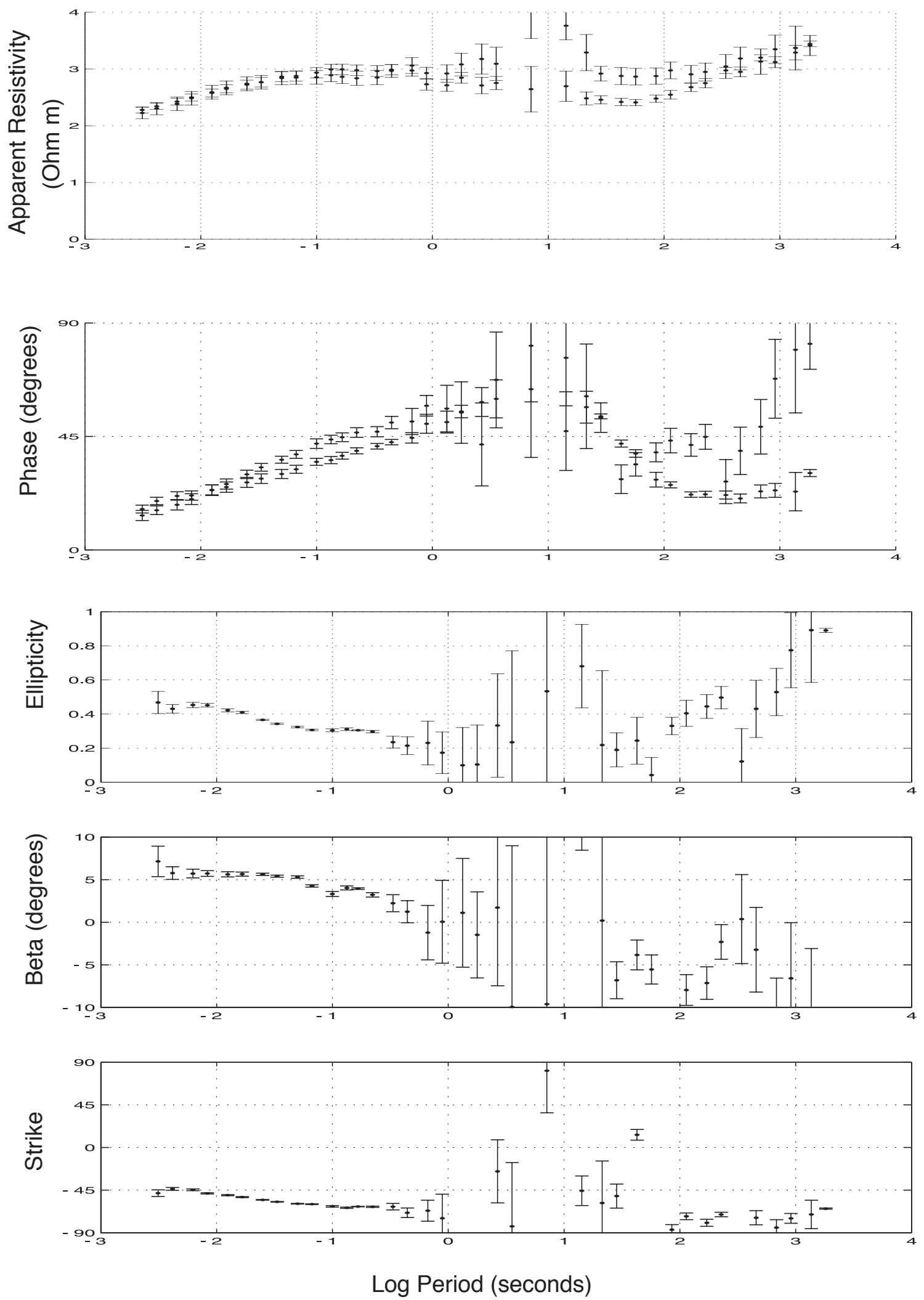

Figure A.22 Data from site MTR-124a 


\section{PHASE TENSOR ELLIPSE MAPS}

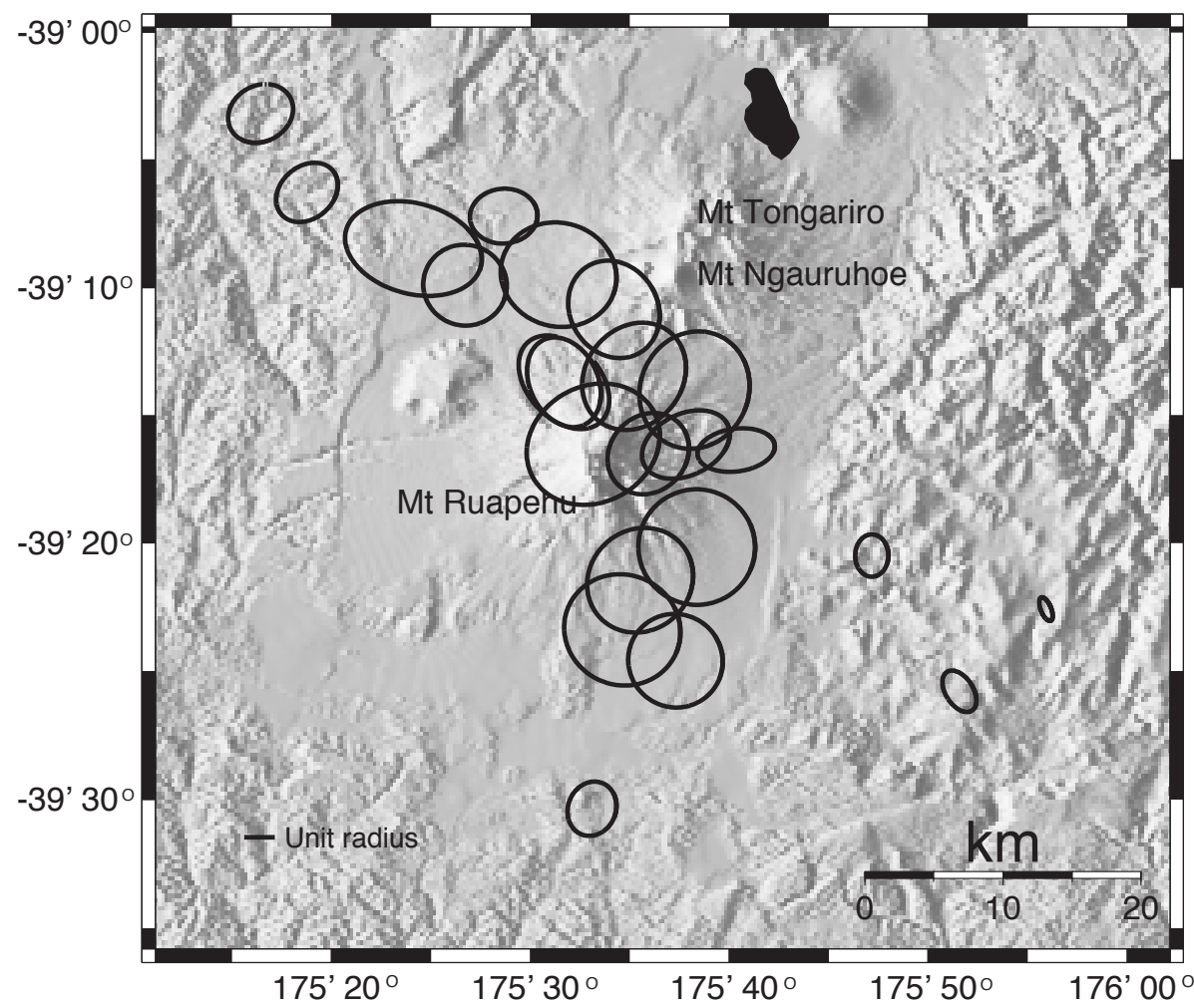

Figure B.1 Phase tensor ellipse map: frequency $=240 \mathrm{~Hz}$ 


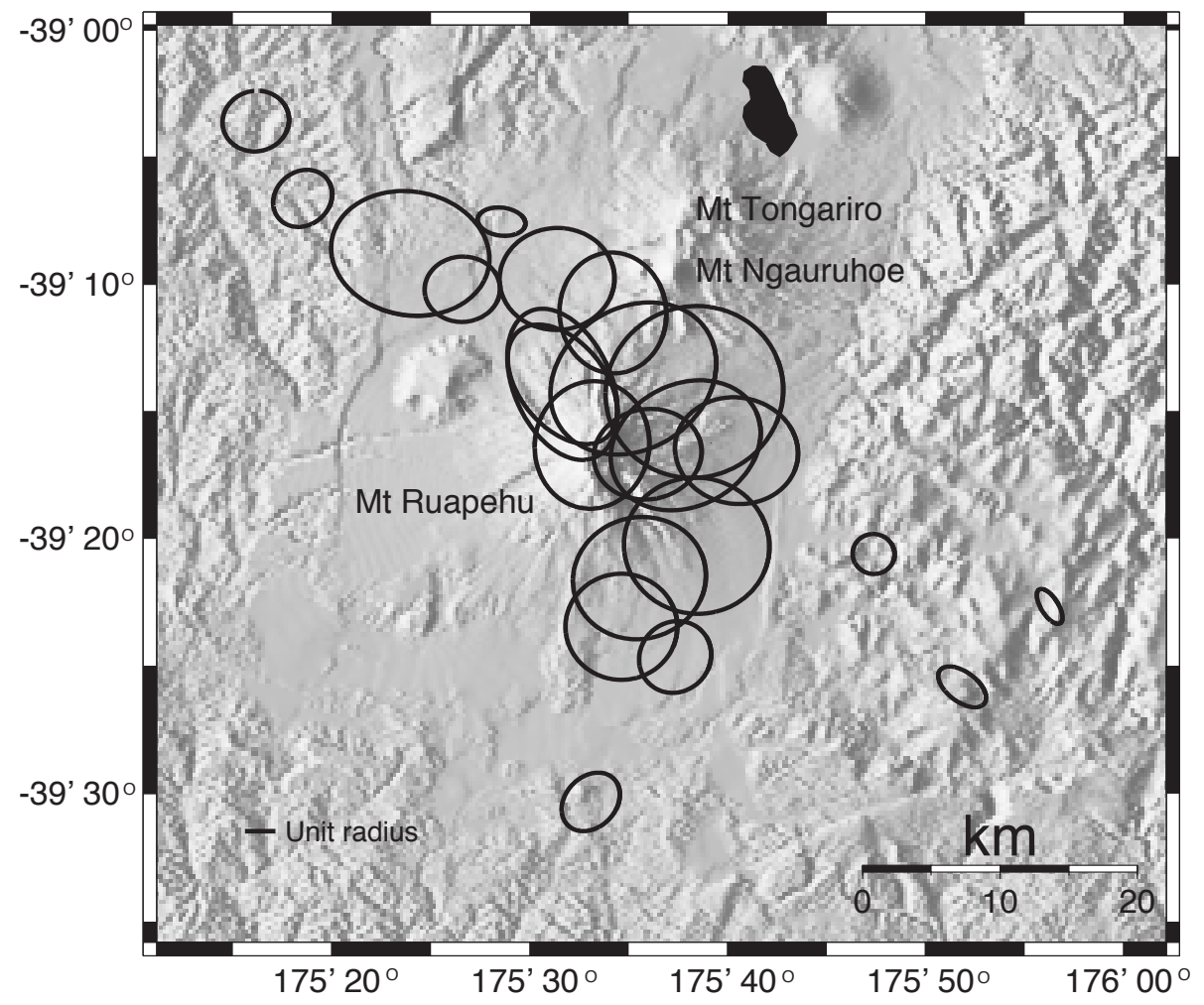

Figure B.2 Phase tensor ellipse map: frequency $=60 \mathrm{~Hz}$

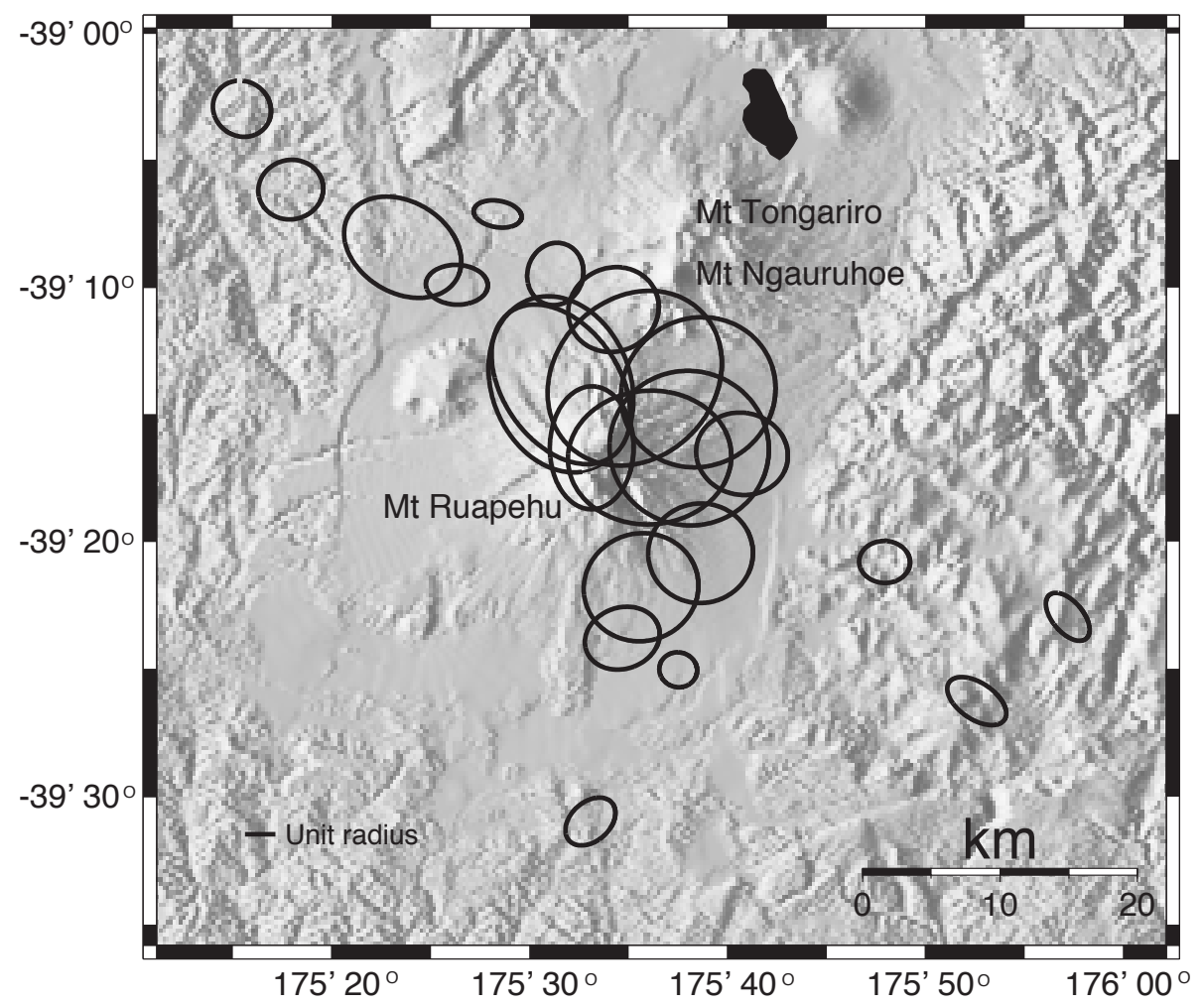

Figure B.3 Phase tensor ellipse map: frequency $=15 \mathrm{~Hz}$ 


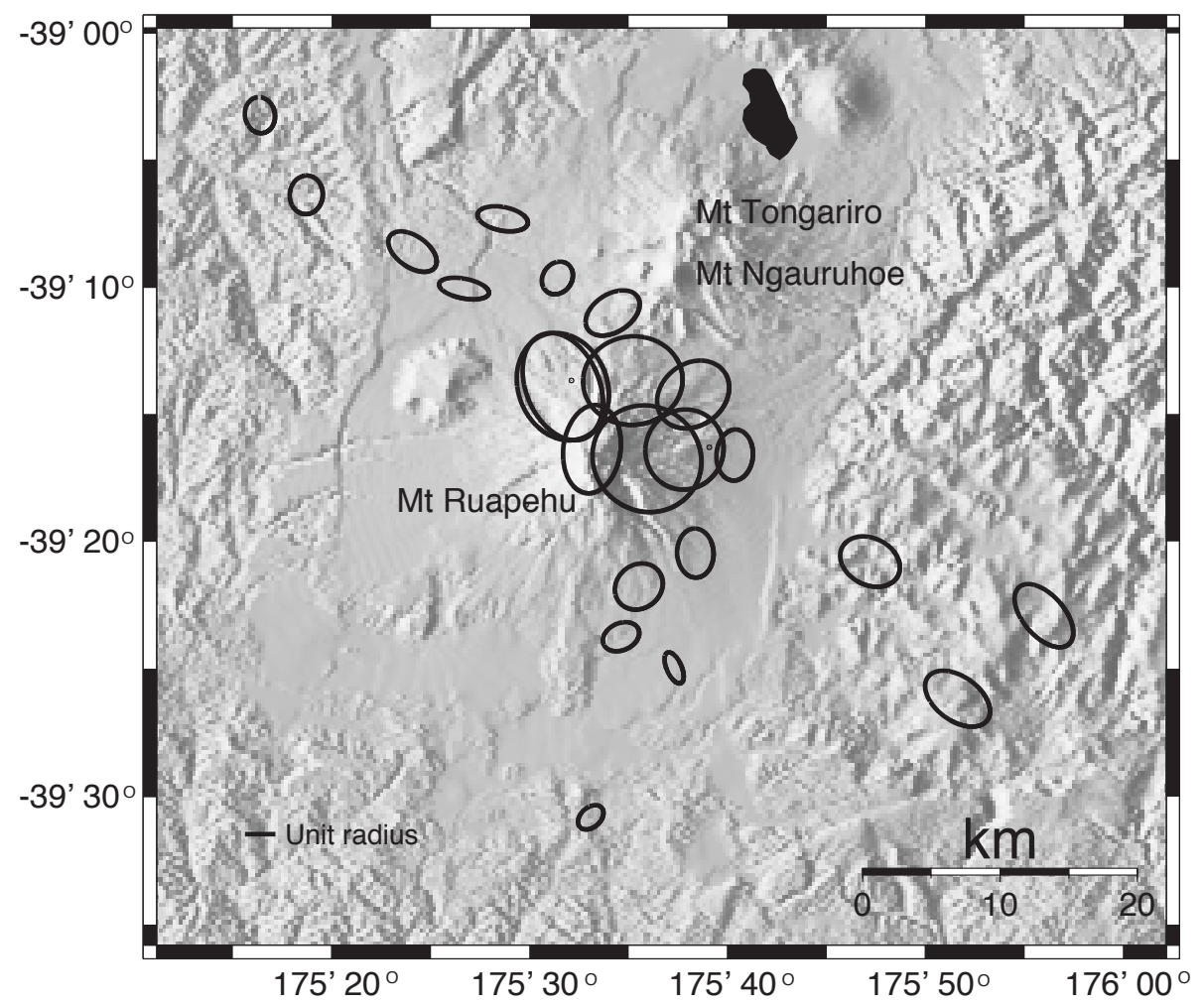

Figure B.4 Phase tensor ellipse map: frequency $=4.5 \mathrm{~Hz}$

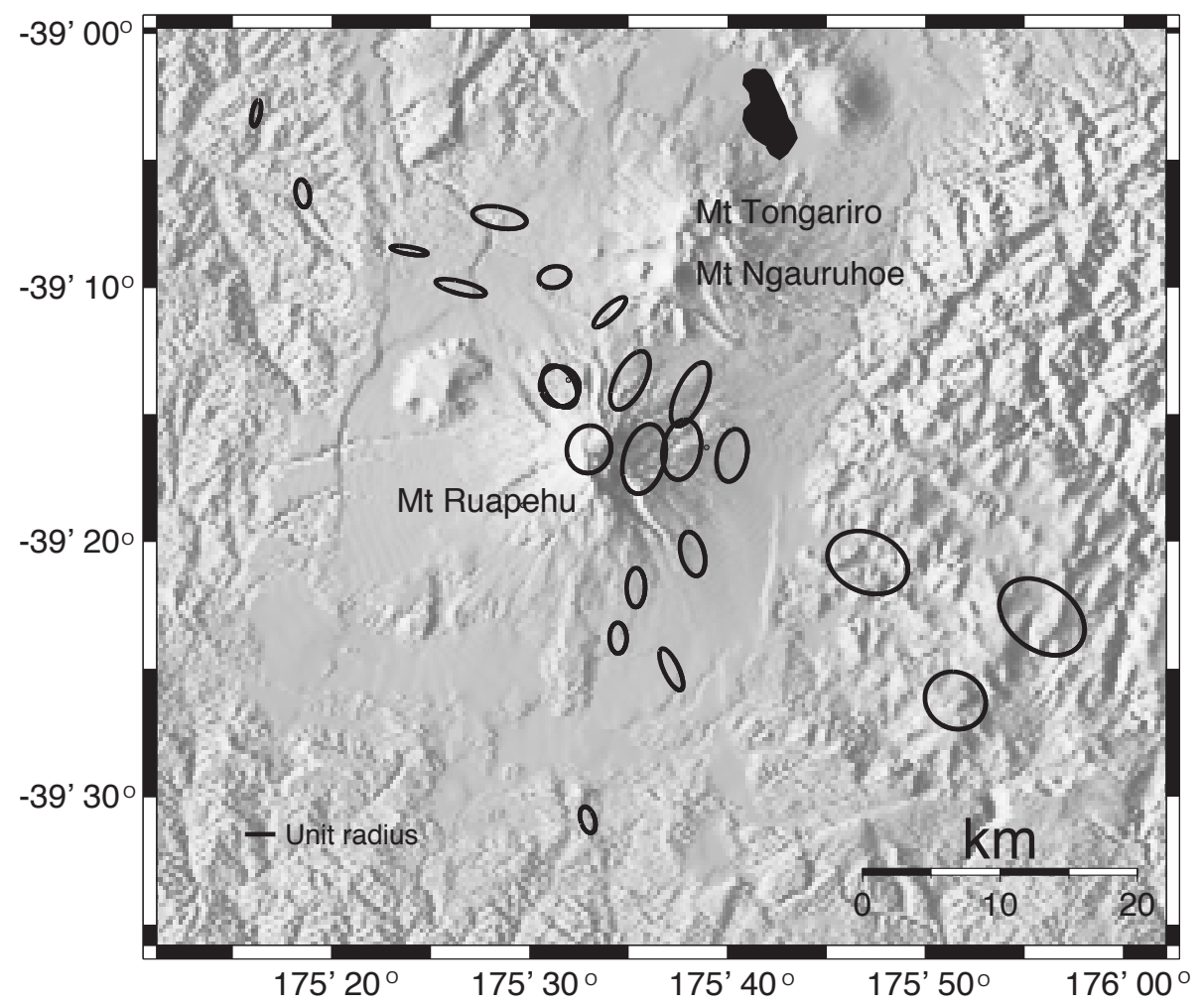

Figure B.5 Phase tensor ellipse map: frequency $=1.125 \mathrm{~Hz}$ 


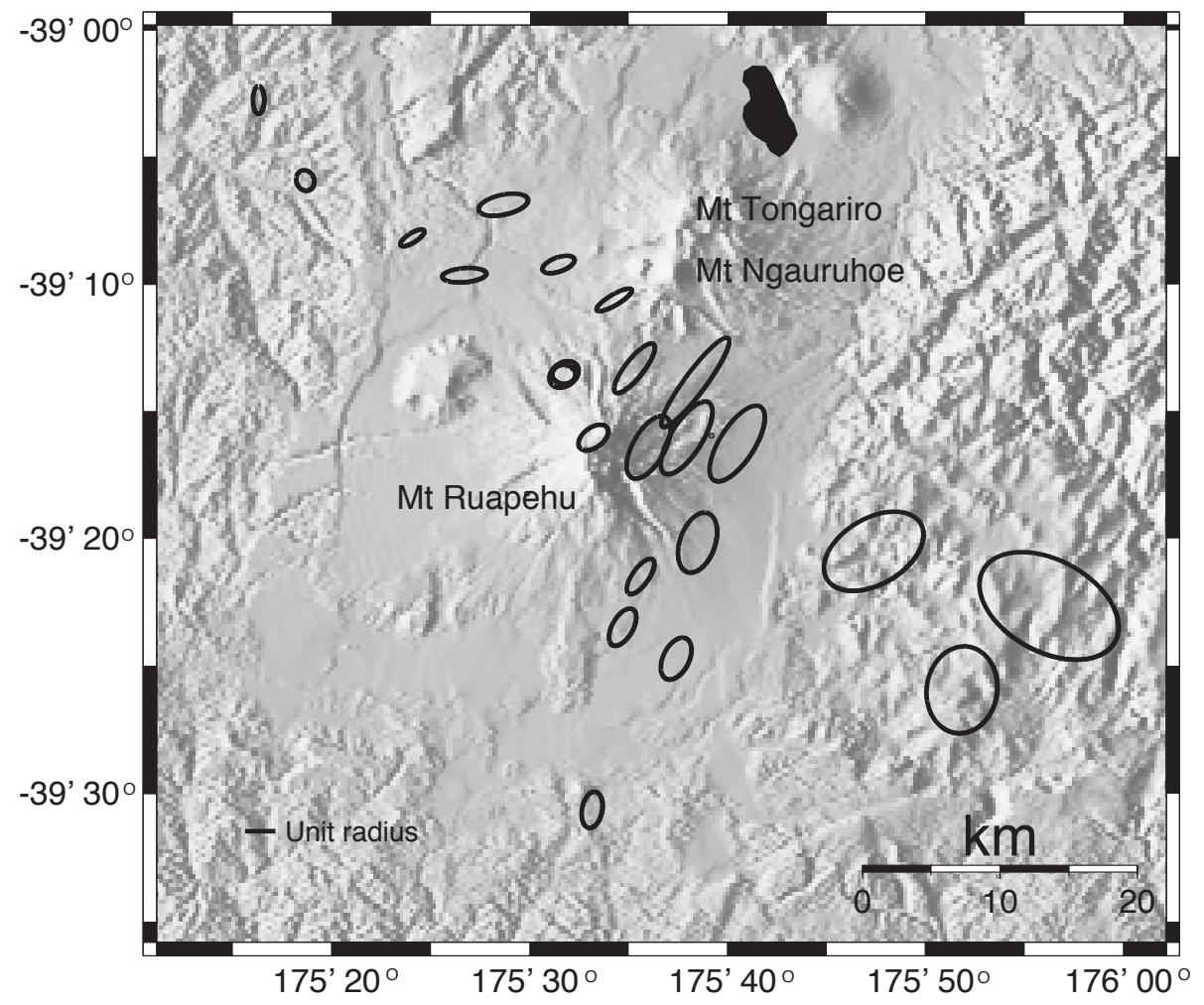

Figure B.6 Phase tensor ellipse map: frequency $=0.281 \mathrm{~Hz}$

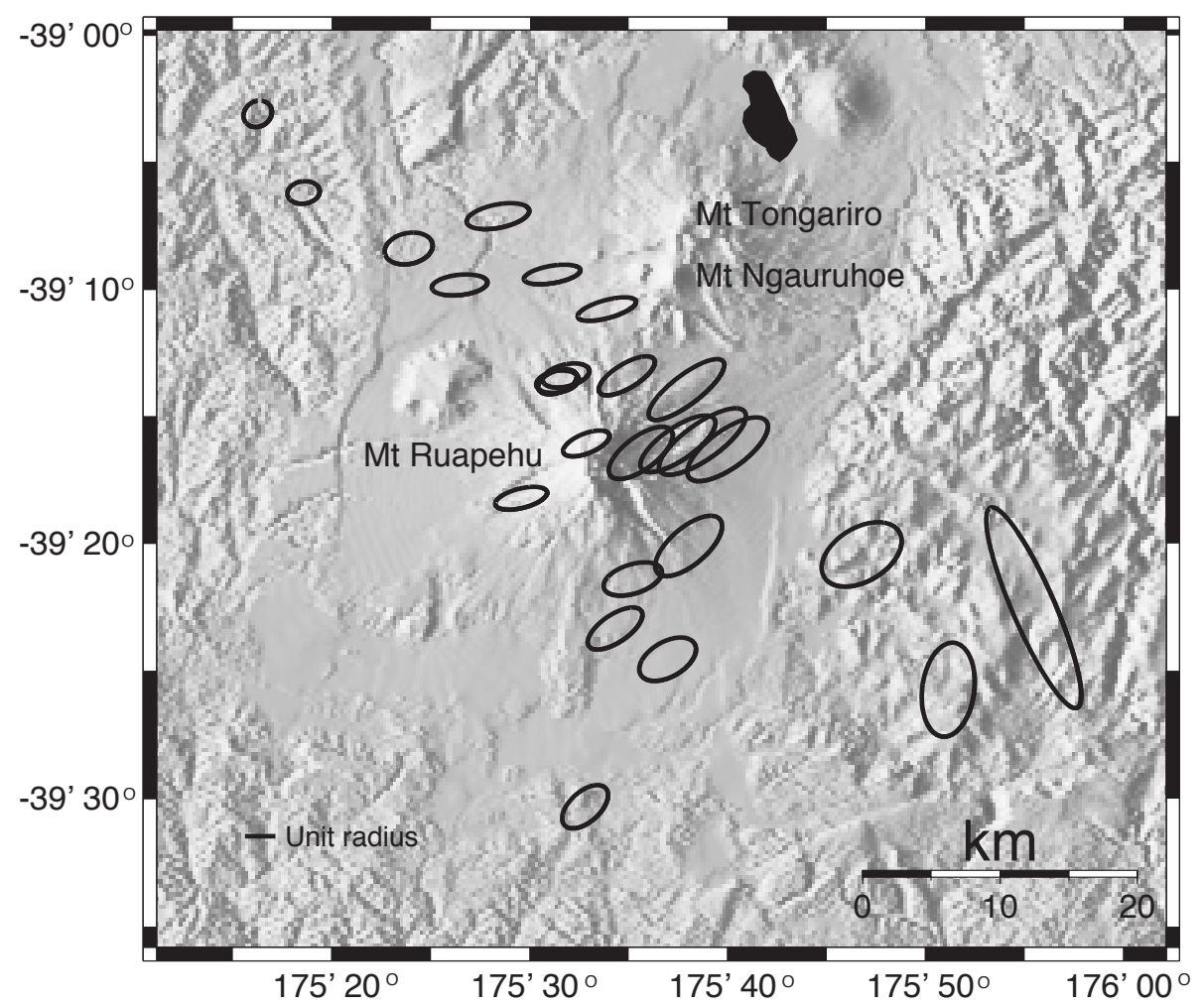

Figure B.7 Phase tensor ellipse map: period $=14.222 \mathrm{~s}$ 


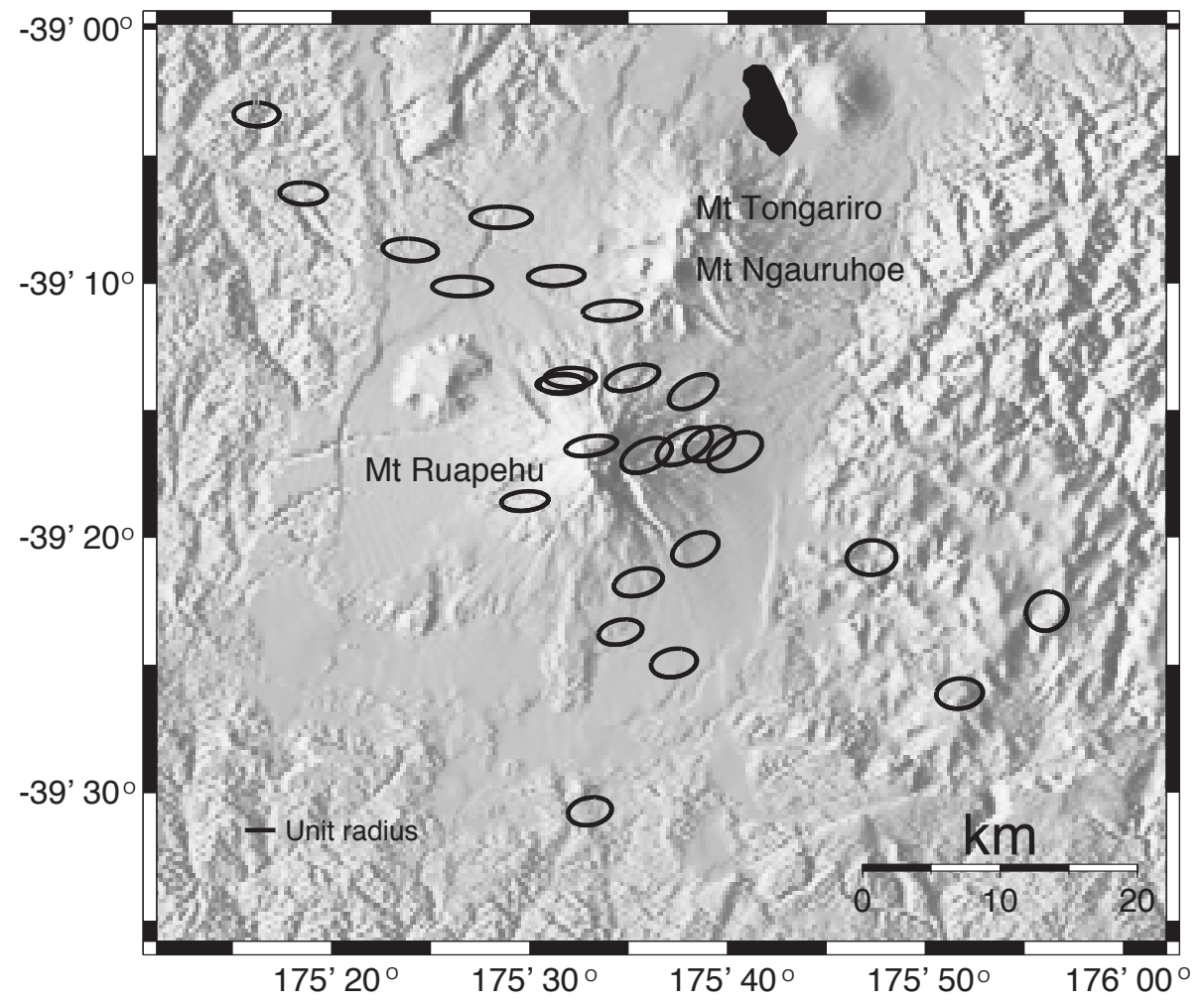

Figure B.8 Phase tensor ellipse map: period $=56.889 \mathrm{~s}$

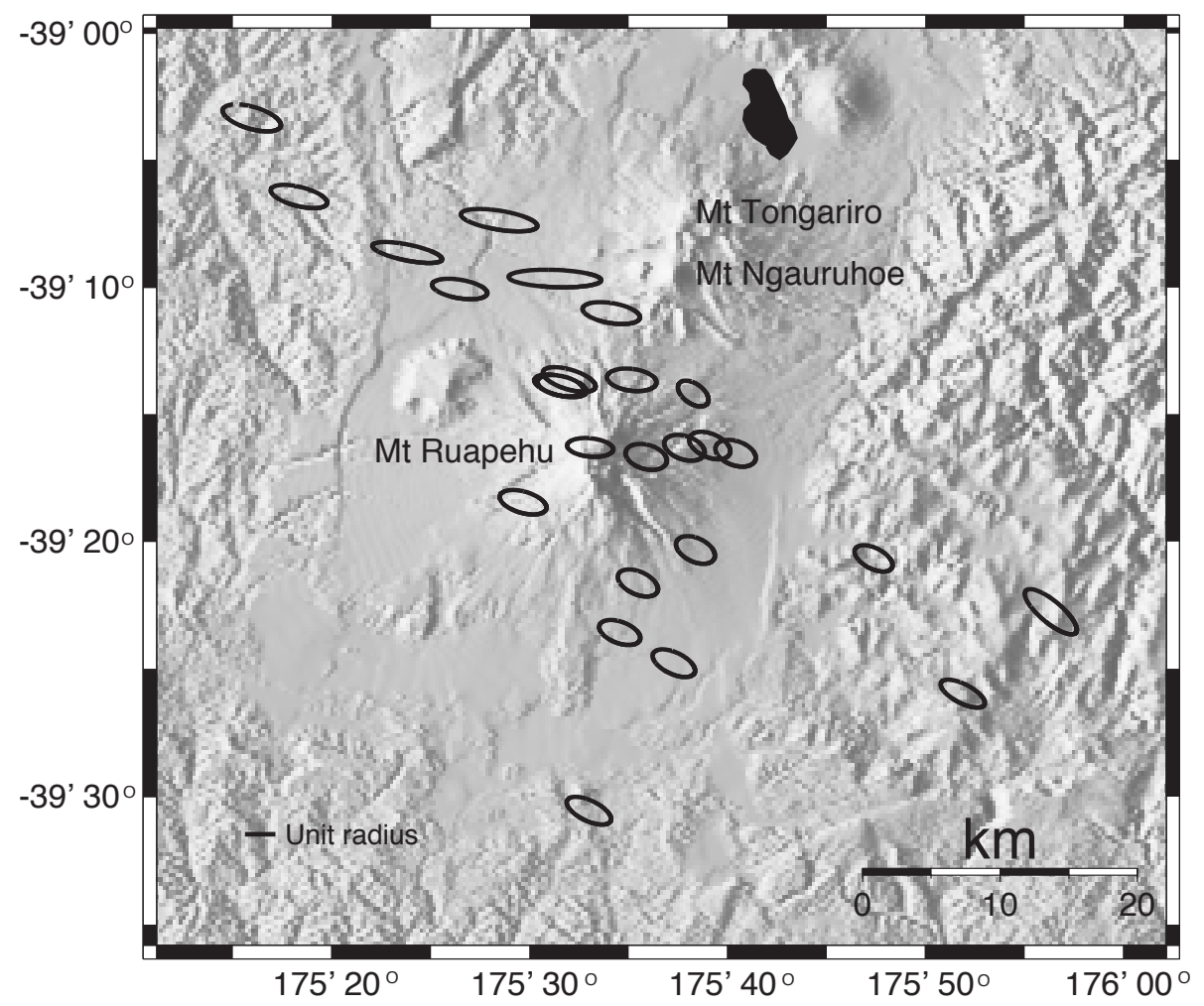

Figure B.9 Phase tensor ellipse map: period $=227.556 \mathrm{~s}$ 


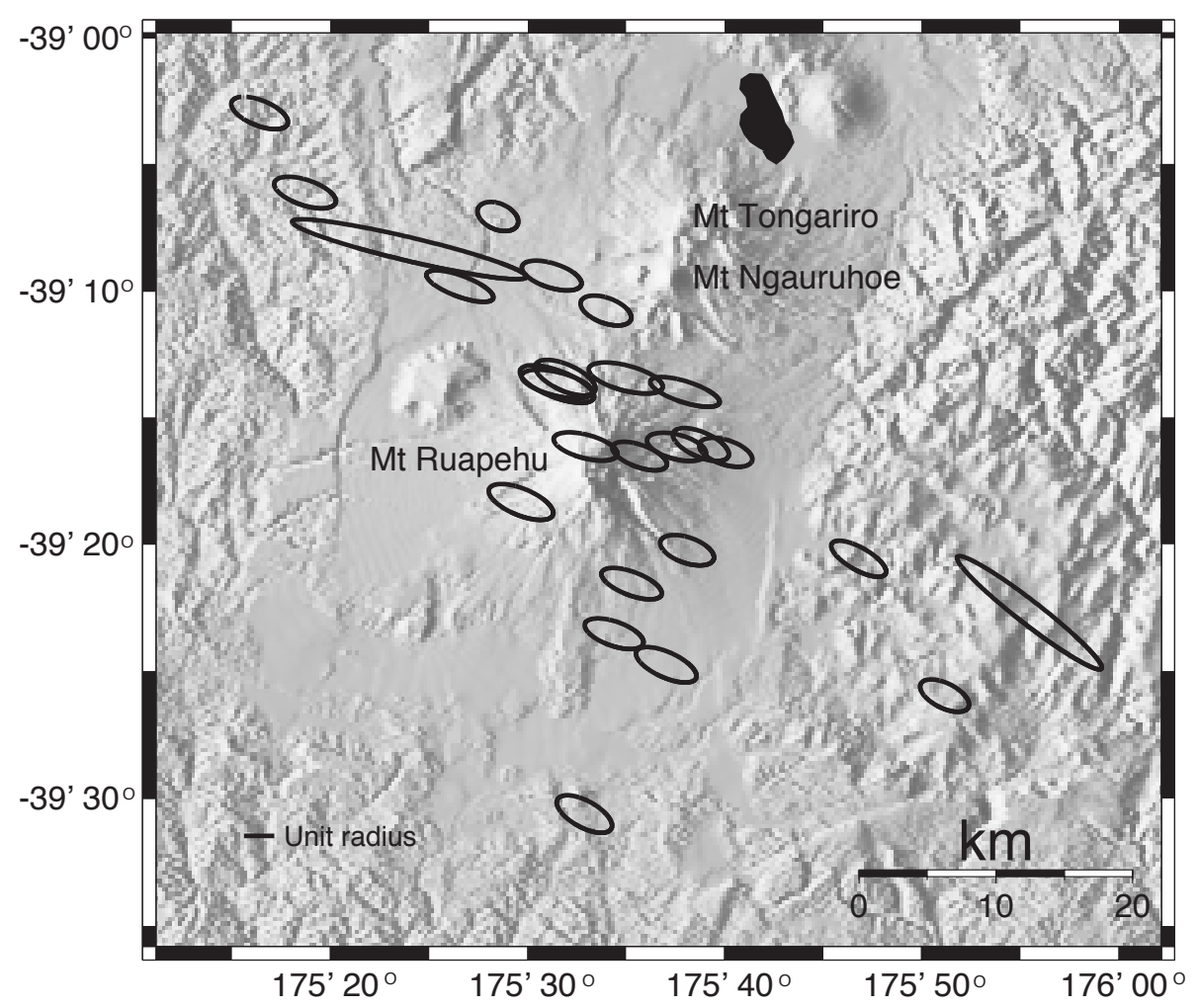

Figure B.10 Phase tensor ellipse map: period $=910.222 \mathrm{~s}$ 


\section{MODEL FITS TO DATA}

\section{C.1 Fit of the 1-D Inversion Models}
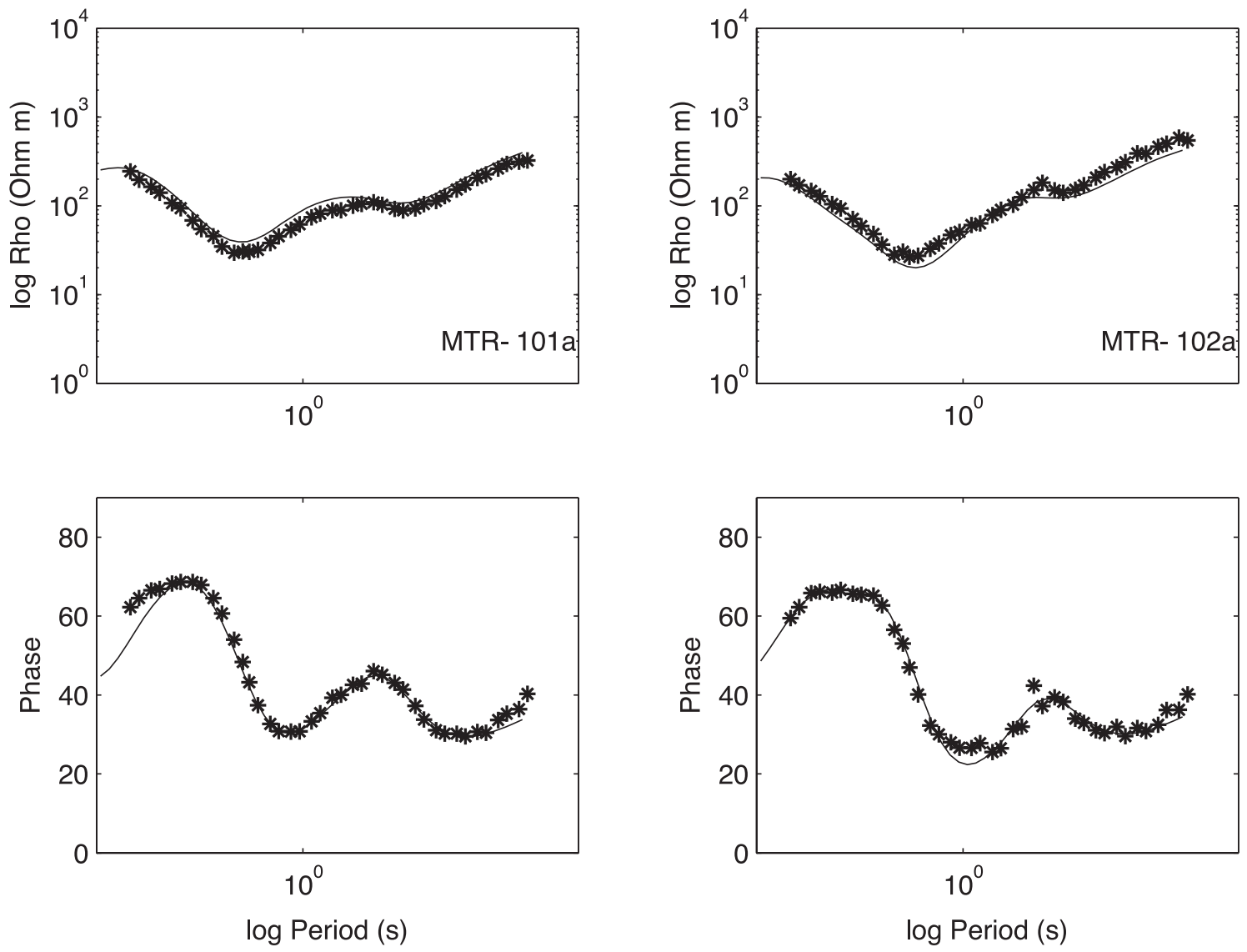

Figure C.1 Theoretical fit of 1-D models (sites MTR-101a to MTR-102a) 

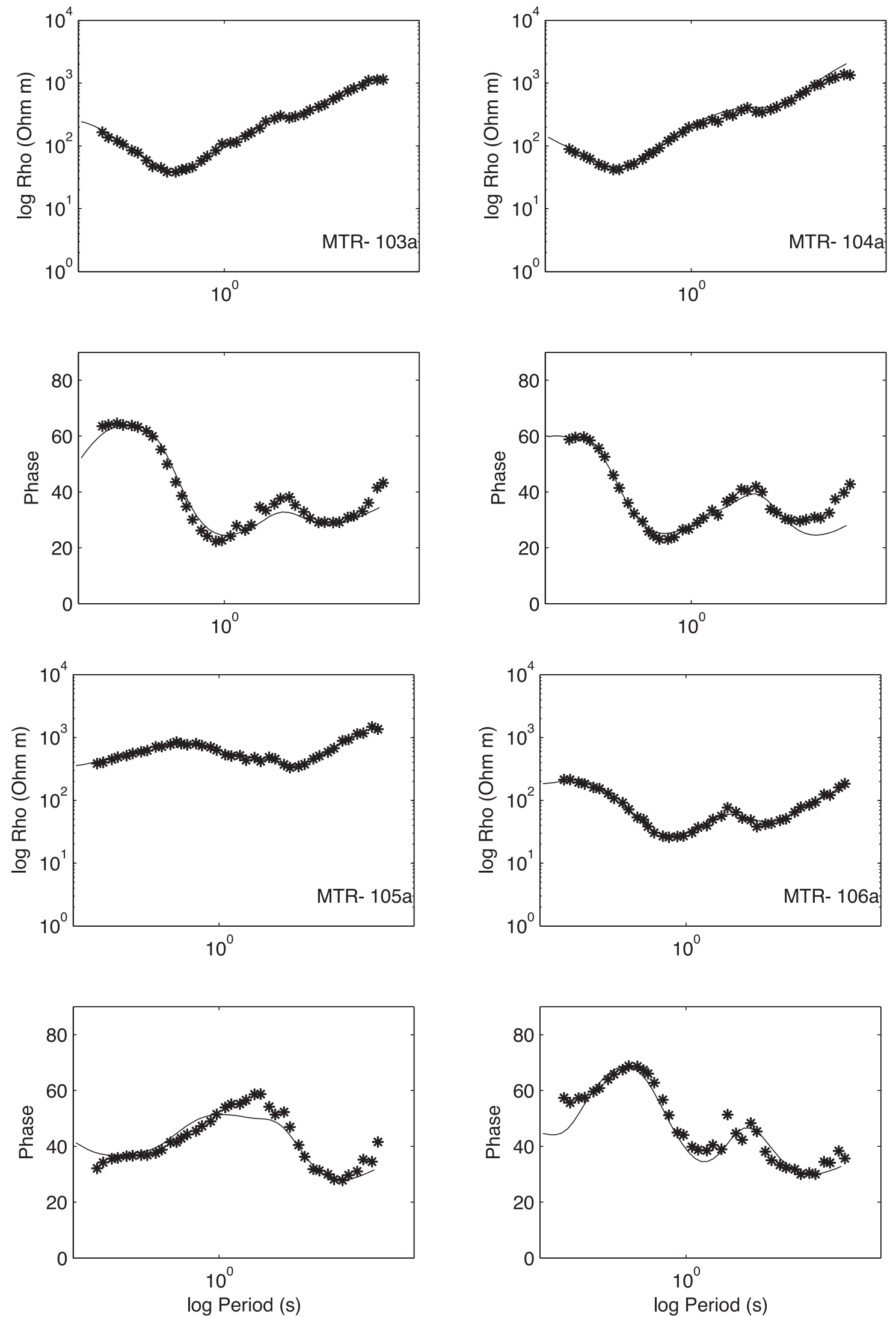

Figure C.2 Theoretical fit of 1-D models (sites MTR-103a to MTR-106a) 

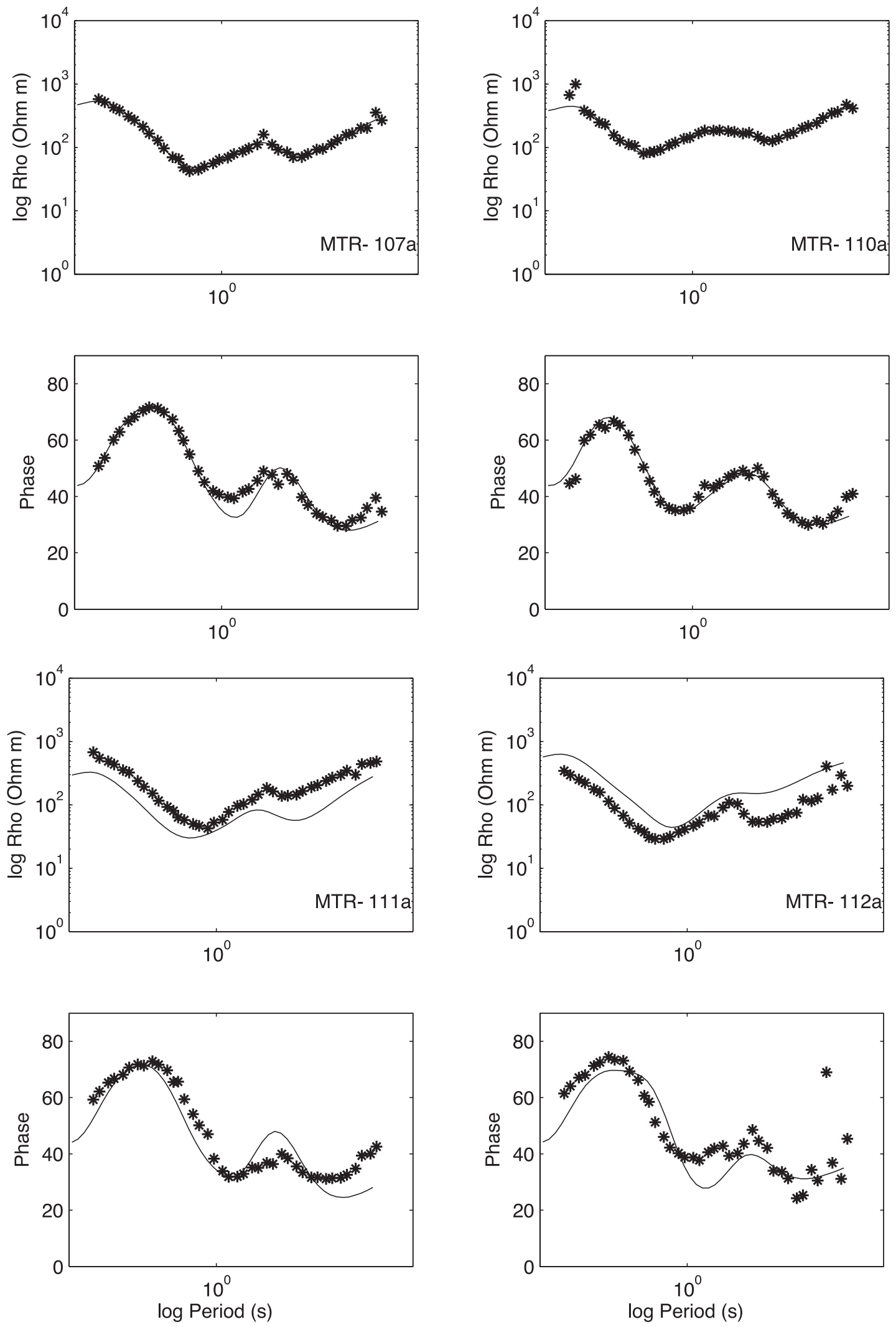

Figure C.3 Theoretical fit of 1-D models (sites MTR-107a to MTR-112a) 

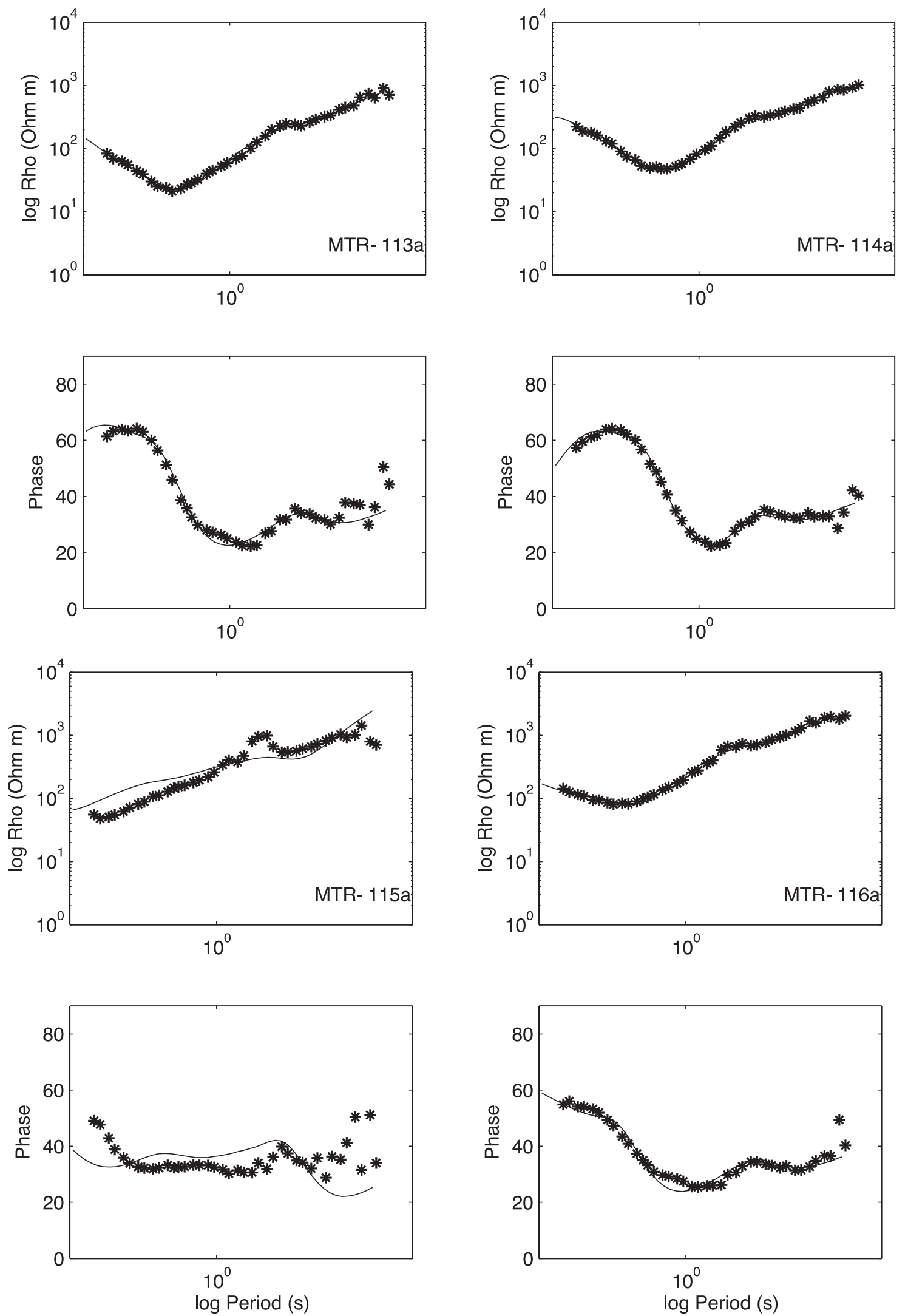

Figure C.4 Theoretical fit of 1-D models (sites MTR-113a to MTR-116a) 

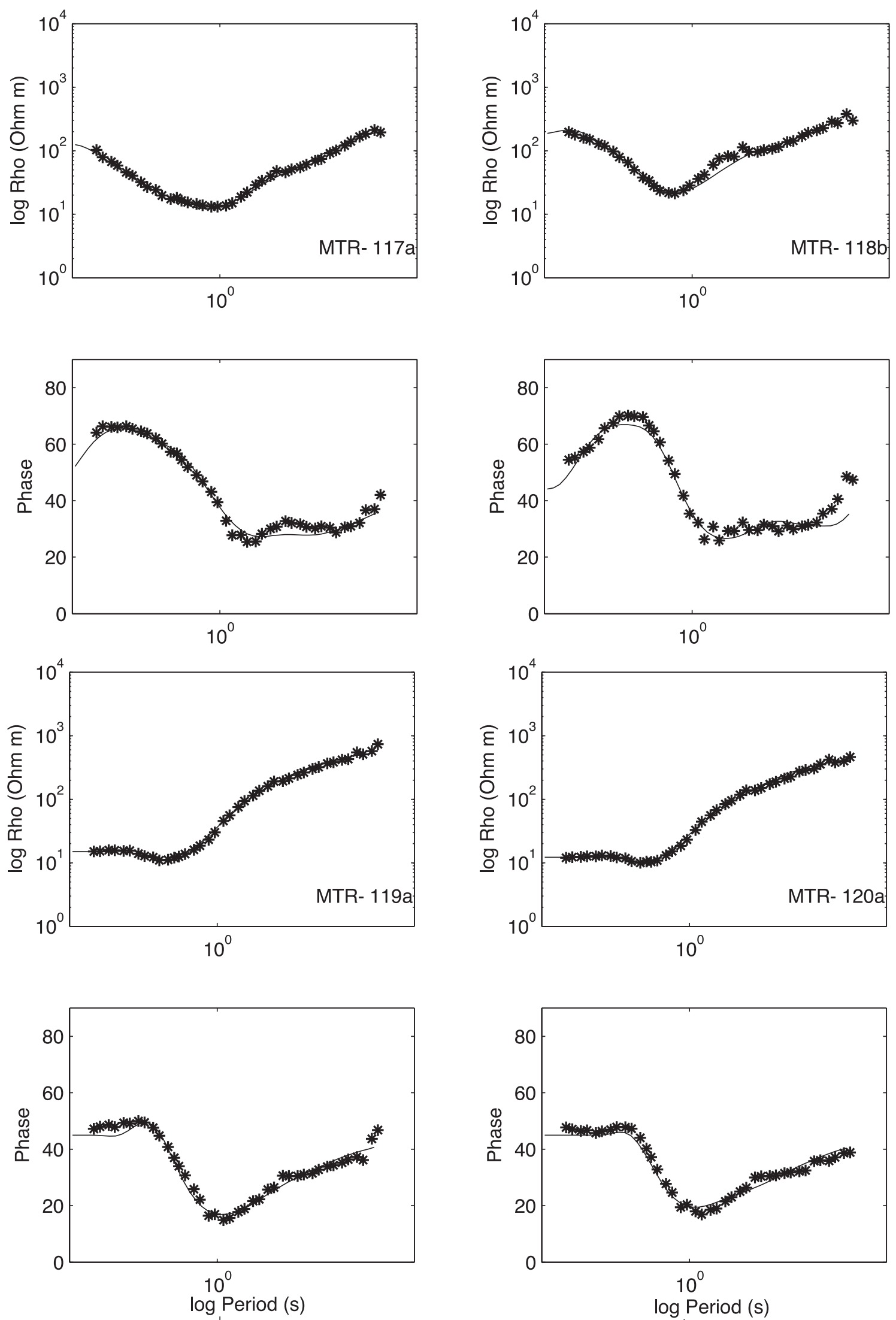

Figure C.5 Theoretical fit of 1-D models (sites MTR-117a to MTR-120a) 

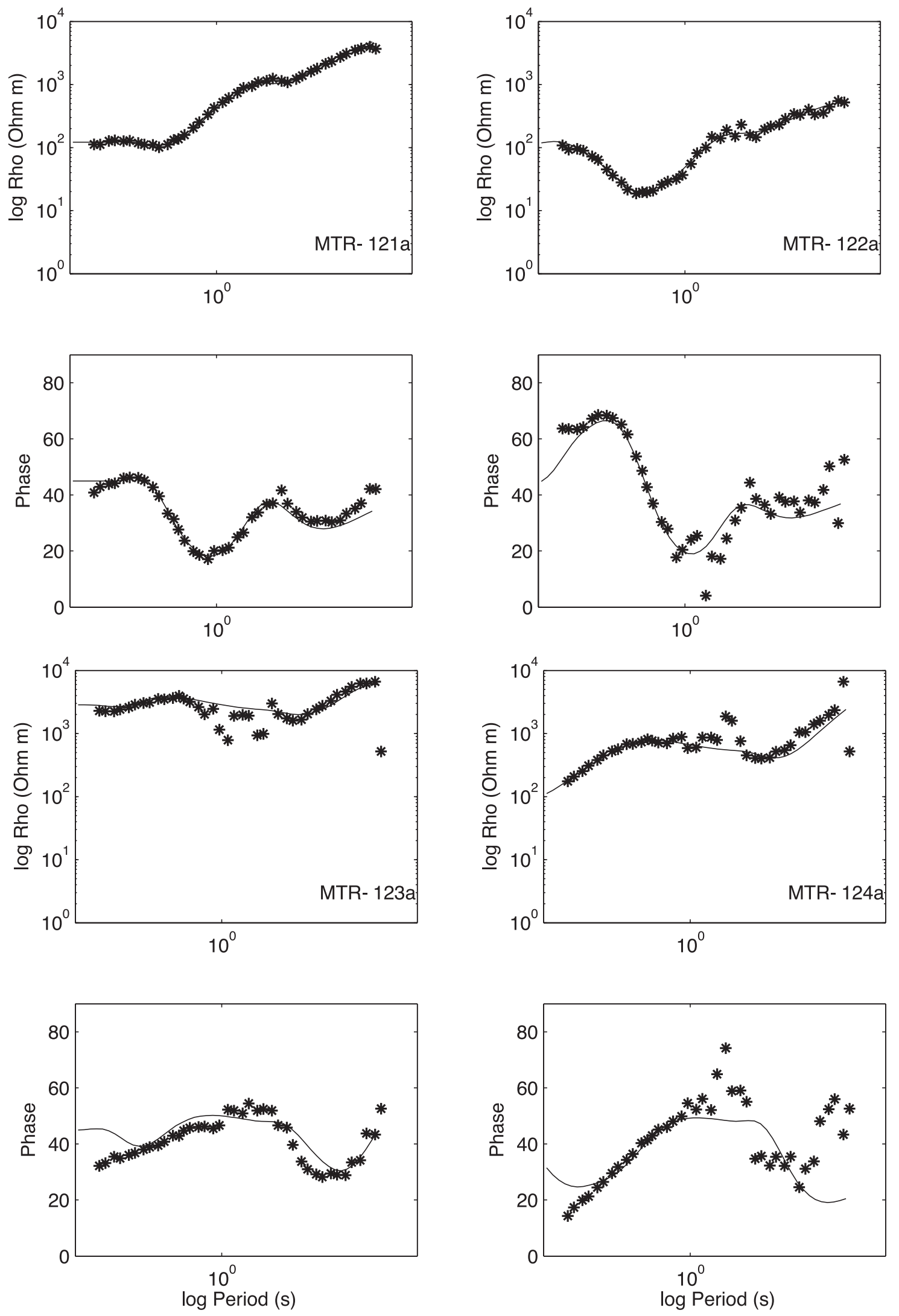

Figure C.6 Theoretical fit of 1-D models (sites MTR-121a to MTR-124a) 


\section{C.2 Fit of the 2-D Inversion Models}
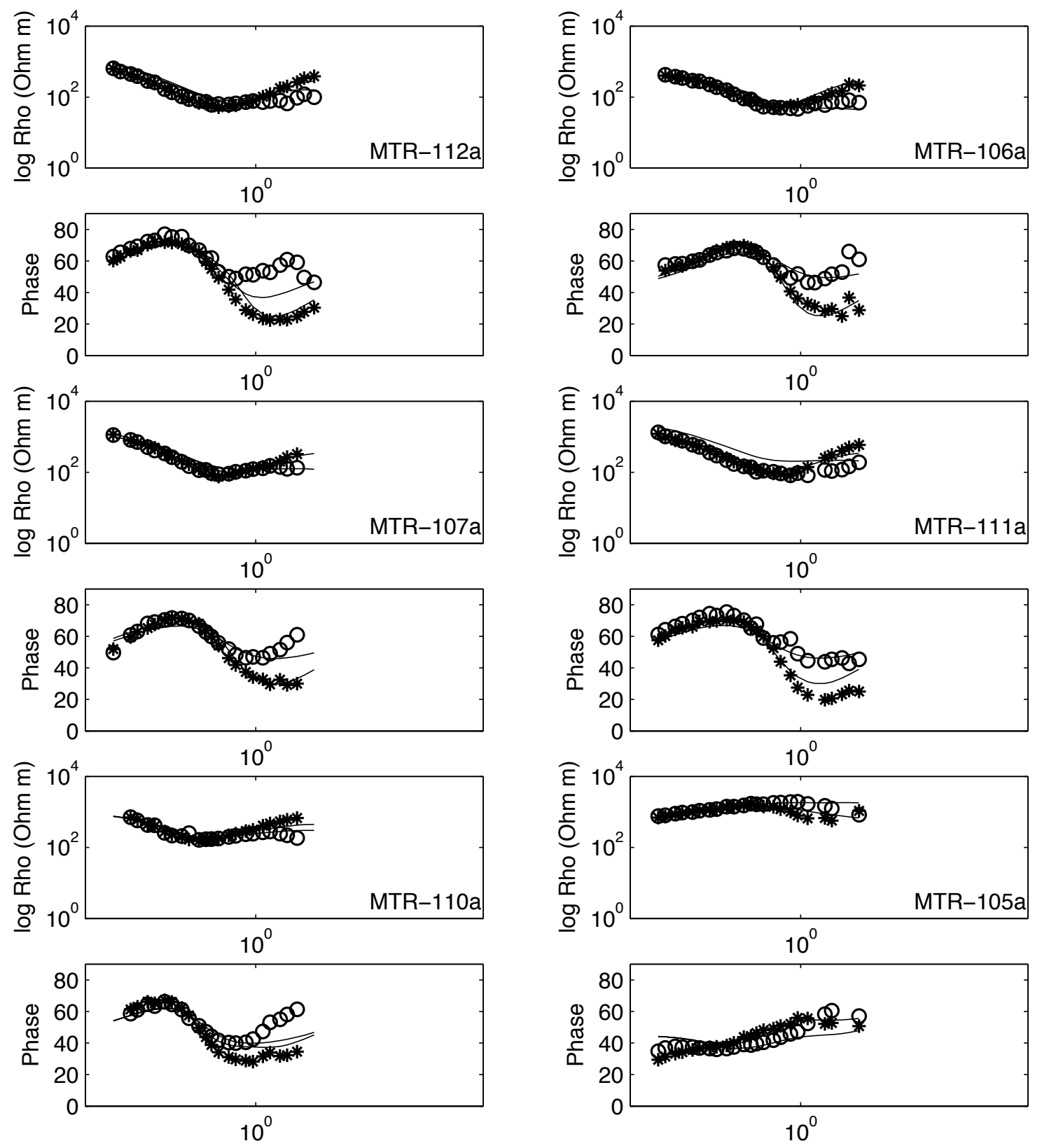

Figure C.7 Theoretical fit of derived data from Profile A to observed data 

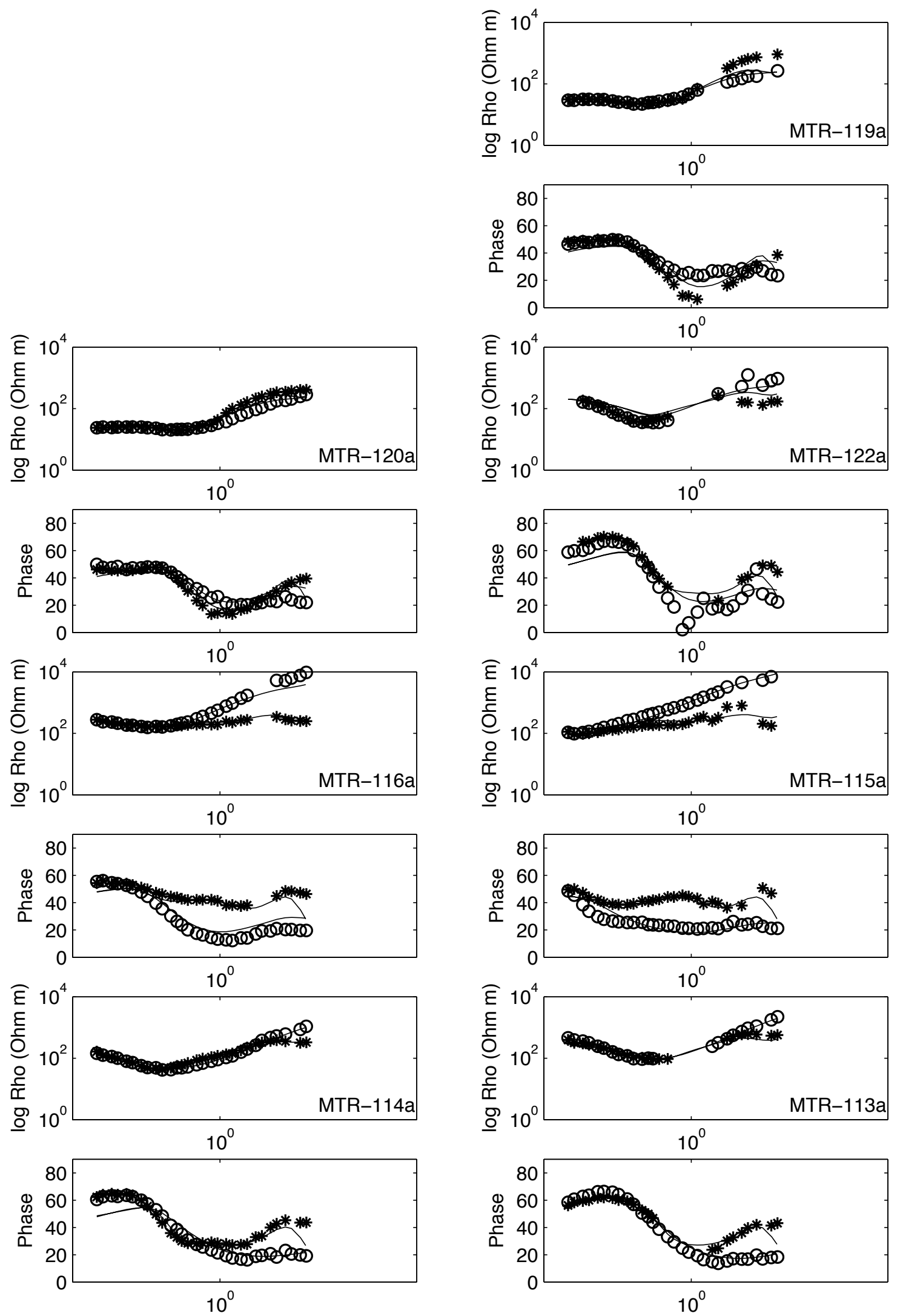

Figure C.8 Theoretical fit of derived data from Profile B to observed data 


\section{C.3 Data fit for 3-D Test}

The comparison between the collected data and the theoretical data derived from the 3-D structure (figures 5.19 and 5.20) created from the 1 and 2-D inversion models. Theoretical data has undergone static shifted for a better fit.
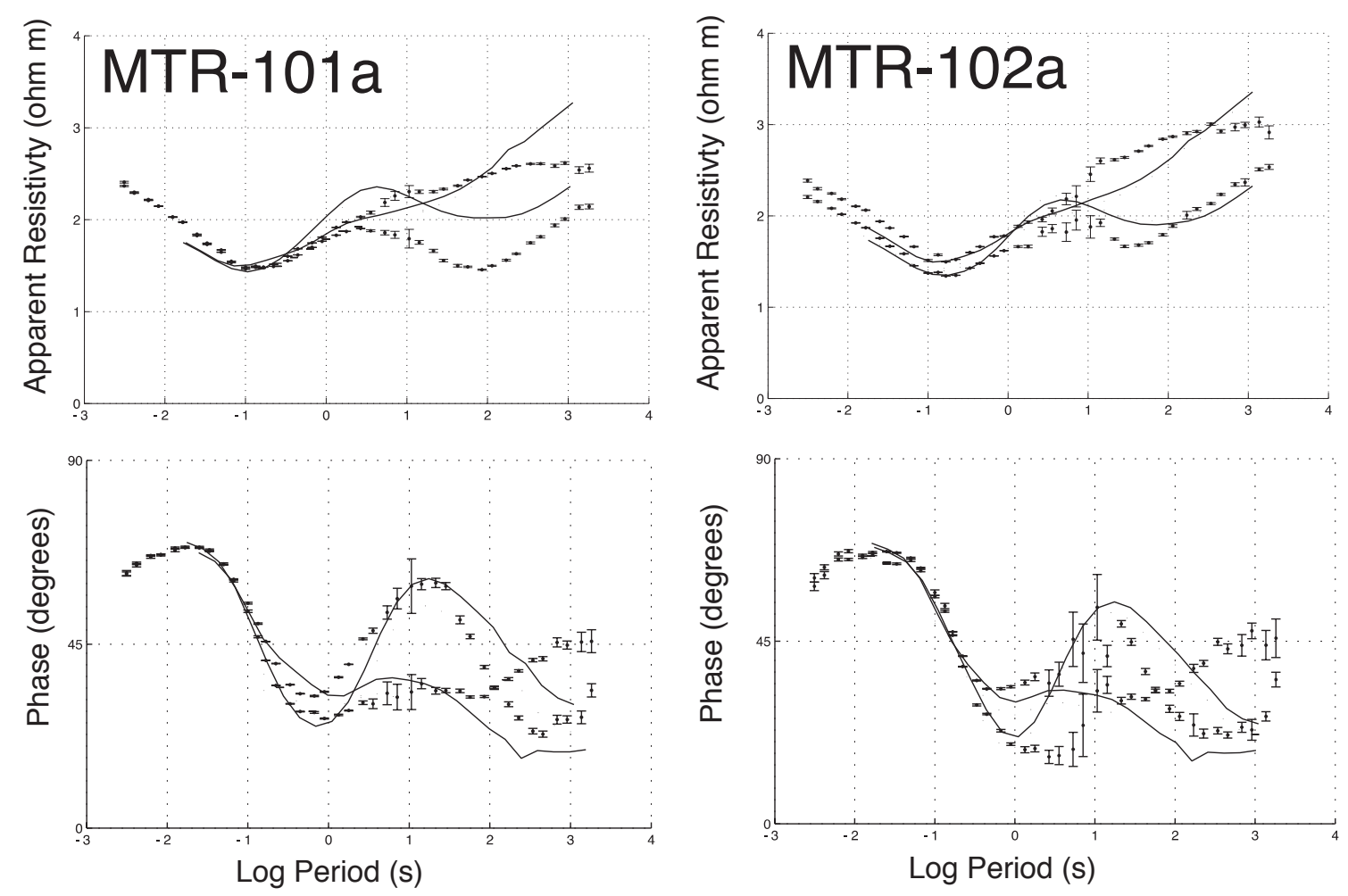

Figure C.9 Theoretical fit of 3-D forward model (sites MTR-101a to MTR-102a) 

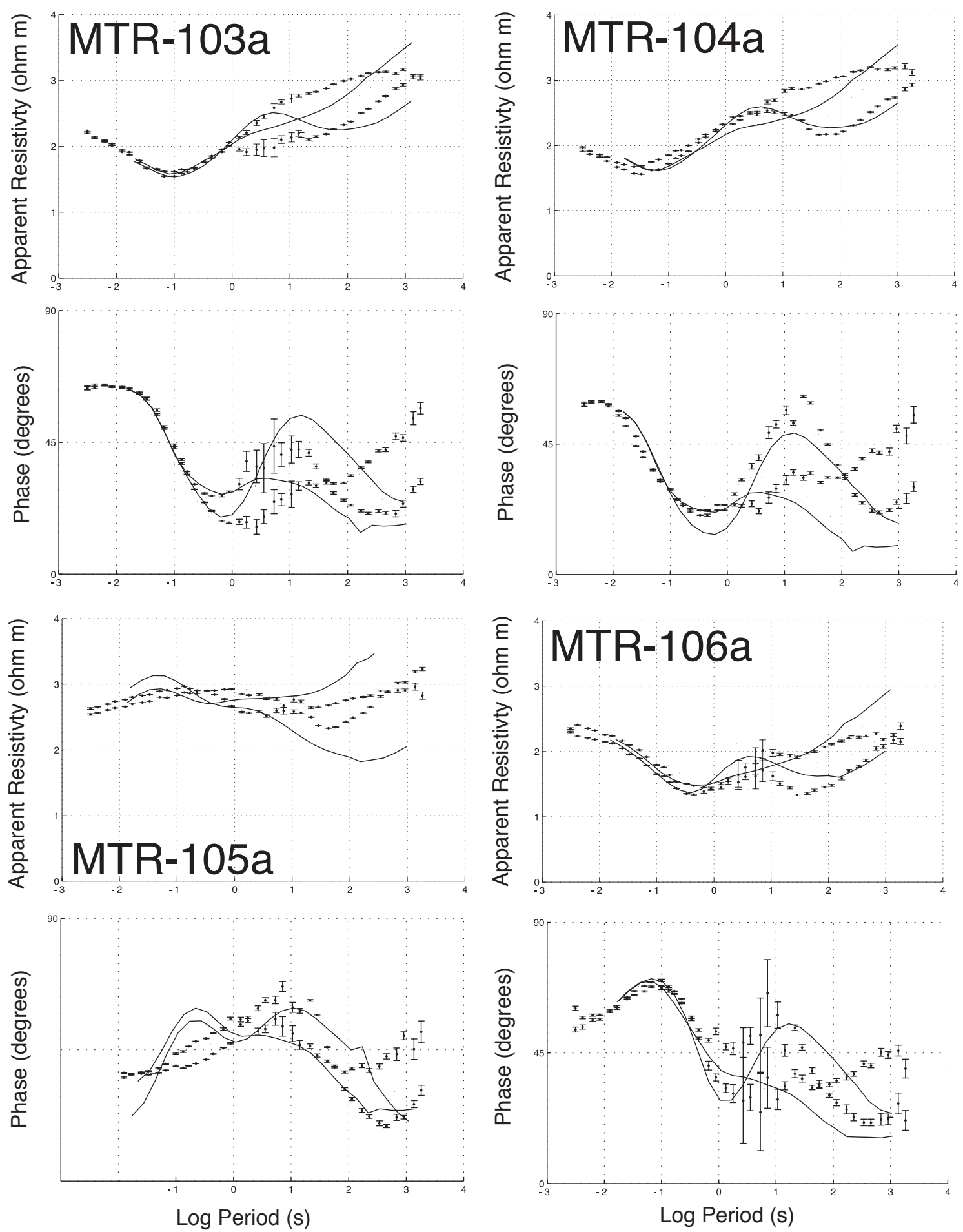

Figure C.10 Theoretical fit of 3-D forward model (sites MTR-103a to MTR-106a) 

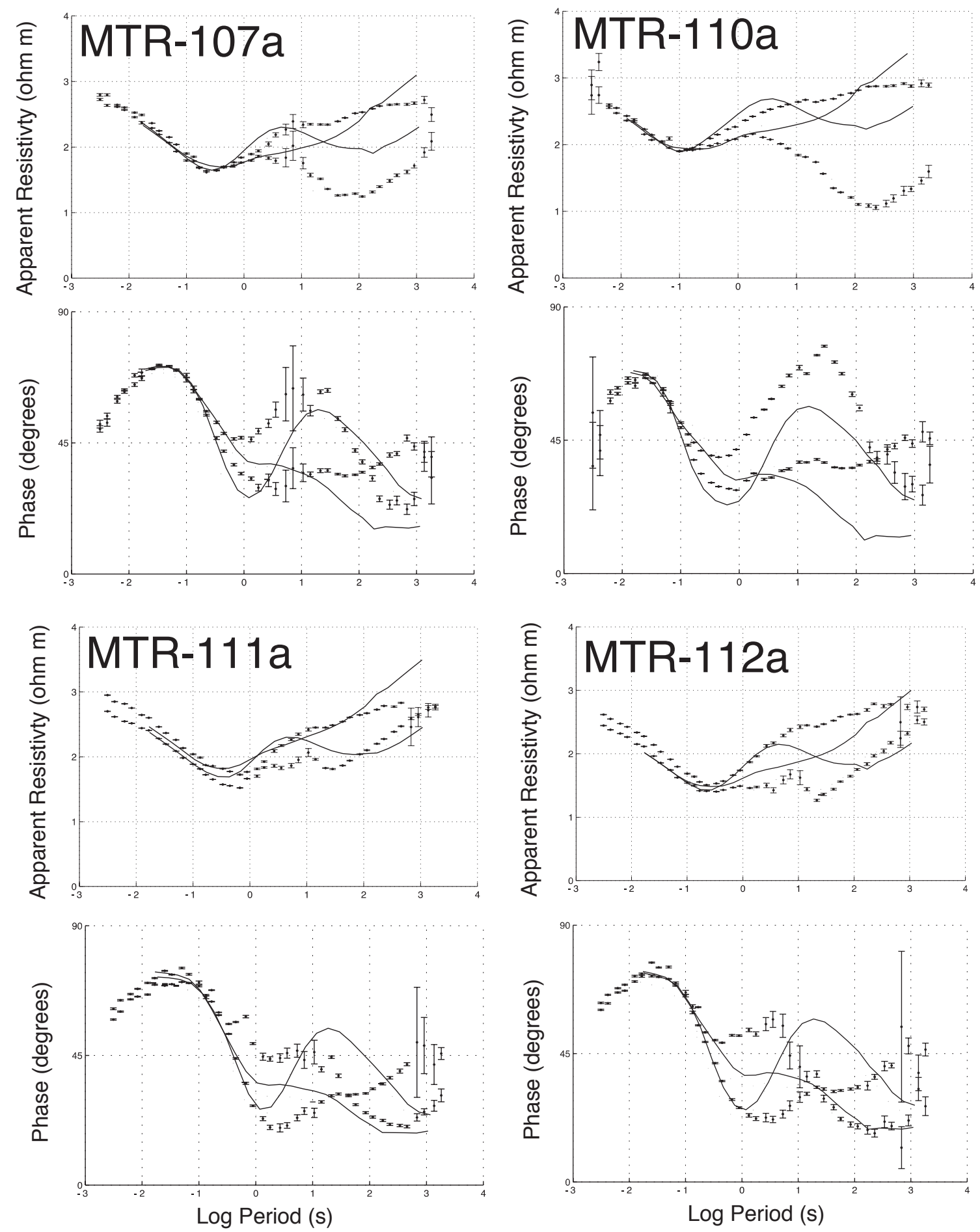

Figure C.11 Theoretical fit of 3-D forward model (sites MTR-107a to MTR-112a) 

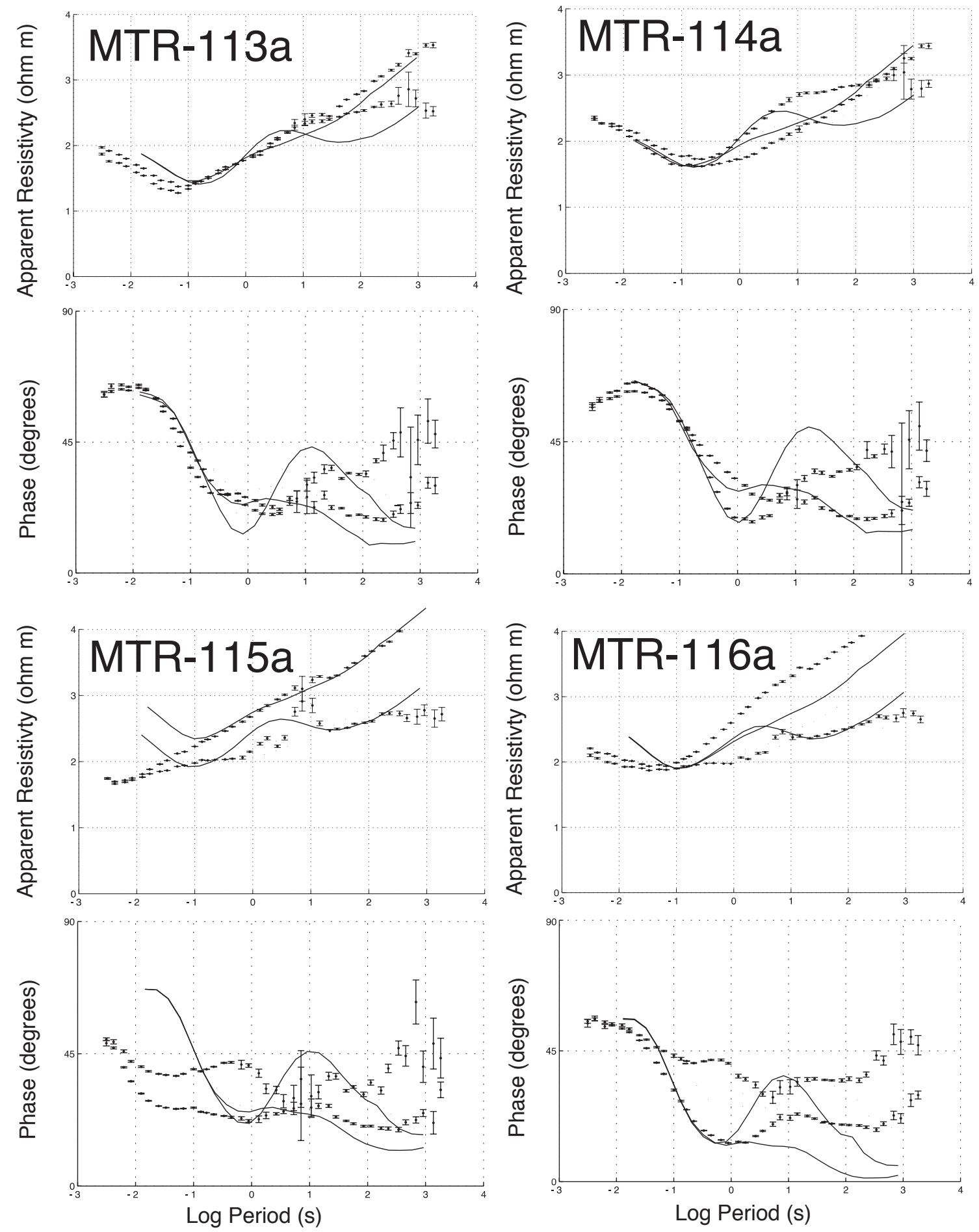

Figure C.12 Theoretical fit of 3-D forward model (sites MTR-113a to MTR-116a) 

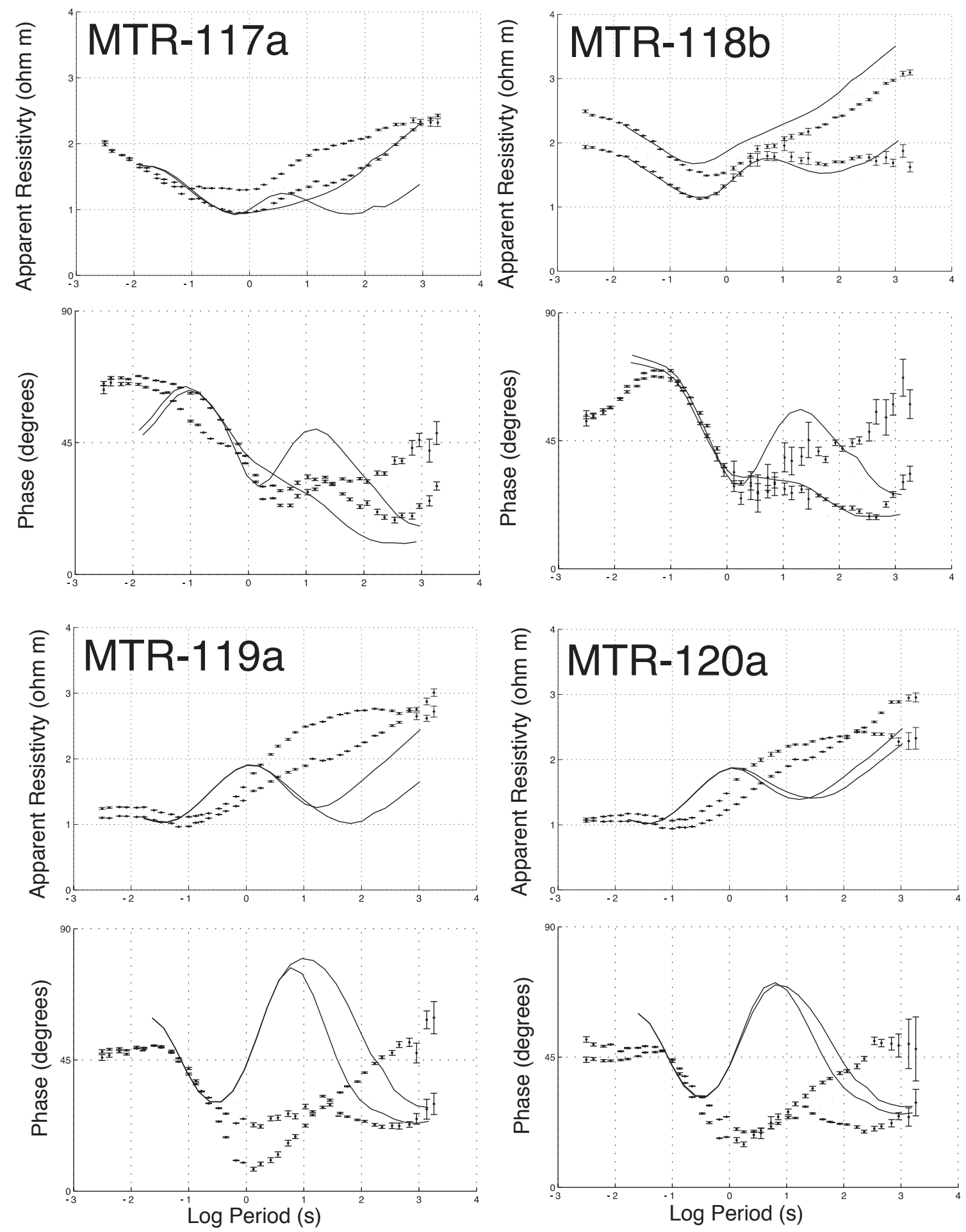

Figure C.13 Theoretical fit of 3-D forward model (sites MTR-117a to MTR-120a) 

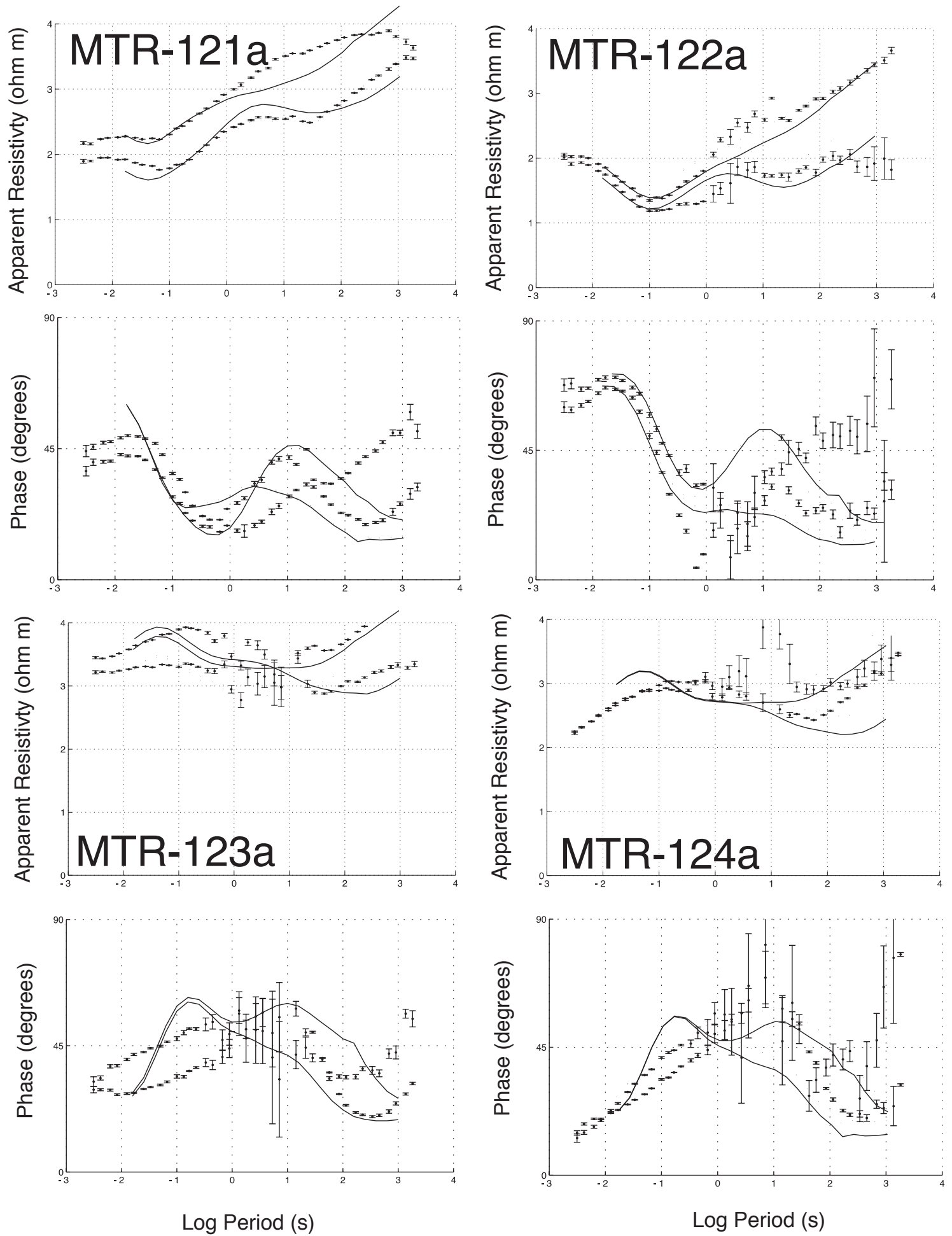

Figure C.14 Theoretical fit of 3-D forward model (sites MTR-121a to MTR-124a) 
SYMBOLS USED

\begin{tabular}{cl}
\hline Symbol & Name \\
\hline$e$ & External \\
$i$ & Internal \\
$n$ & Normal \\
$t$ & Tangential \\
$I$ & Imaginary \\
$R$ & Real \\
$\alpha$ & Azimuth angle \\
$\alpha$ & 1-D arbitrary variable \\
$\beta$ & Skew \\
$\delta$ & Skin depth \\
$\epsilon_{0}$ & Permittivity \\
$\phi$ & Longitude \\
$\mu_{0}$ & Magnetic permeability \\
$\theta$ & Latitude (in section 3.1 .1$)$ \\
$\theta$ & Rotation angle of measurement axes \\
$\rho_{a}$ & Apparent resistivity \\
$\sigma$ & Conductivity \\
$\tau$ & Period \\
$v$ & Wave number \\
$\omega$ & Angular frequency \\
$\zeta$ & Degree of saturation \\
$\Phi$ & Phase \\
$\Phi_{m a x}$ & Major principal axes \\
$\Phi_{m i n}$ & Minor principal axes \\
$\Psi$ & Any function of $x, y$ and $z$ \\
$a$ & Distance from centre of sphere \\
$f$ & The volume fraction of the better conductor \\
$g$ & Static shift \\
$h$ & Depth \\
$i$ & Imaginary number \\
$k^{2}$ & Simplification: $k^{2}=-i \omega \mu_{0} \sigma$ \\
$n$ & Harmonic number \\
& \\
\hline &
\end{tabular}




\begin{tabular}{|c|c|}
\hline Symbol & Name \\
\hline$q$ & Charge \\
\hline$r$ & Radius \\
\hline$t$ & Time \\
\hline$x$ & North-south direction \\
\hline$y$ & East-west direction \\
\hline$z$ & Depth \\
\hline$C$ & Constant derived from Laplace's equation \\
\hline$D$ & East-west magnetic field \\
\hline$E$ & East-west electric field \\
\hline$F$ & Generic function of poloidal field \\
\hline$H$ & North-south magnetic field \\
\hline$N$ & North-south electric field \\
\hline$P$ & Porosity of a rock \\
\hline$S$ & Skew dimensionality \\
\hline$S_{n}^{m}$ & Spherical harmonic terms (section 3.1.1) \\
\hline$T$ & Period \\
\hline$\underline{j}$ & Current density \\
\hline $\bar{j}_{s}$ & Surface current \\
\hline$\underline{A}$ & Internal magnetic field \\
\hline$\underline{B}$ & Magnetic field \\
\hline$\underline{E}$ & Electric field \\
\hline$\underline{H}$ & Measured magnetic field \\
\hline$\underline{U}$ & External magnetic field \\
\hline$\underline{X}$ & Real impedance component \\
\hline$\underline{Y}$ & Imaginary impedance component \\
\hline$\underline{\underline{A}}$ & Anisotropy matrix \\
\hline$\overline{\bar{C}}$ & Distortion matrix \\
\hline$\underline{\underline{R}}$ & Rotation matrix \\
\hline$\underline{\underline{S}}$ & Shear matrix \\
\hline$\underline{\underline{\underline{T}}}$ & Twist matrix \\
\hline$\underline{\bar{Z}}$ & Impedance tensor \\
\hline$\underline{\underline{\underline{Z}}}_{\|}$ & Impedance parallel to strike \\
\hline$\underline{\underline{Z}}_{\perp}$ & Impedance perpendicular to strike \\
\hline$\overline{\bar{\partial}} \frac{1}{l}$ & Line derivative \\
\hline$\partial S$ & Surface derivative \\
\hline$\partial V$ & Volumetric derivative \\
\hline$\frac{\partial \underline{E}}{\partial t}$ & Displacement current \\
\hline
\end{tabular}

